\title{
Scanning tunneling spectroscopy of space charge regions in semiconductors: \\ From single donor to heterostructure systems
}

\author{
Dissertation \\ zur Erlangung des mathematisch-naturwissenschaftlichen Doktorgrades \\ "Doctor rerum naturalium" \\ der Georg-August-Universität Göttingen
}

vorgelegt von

\section{Karen Teichmann}

aus Gießen 
Referent: Prof. Dr. R. G. Ulbrich

Koreferent: PD Dr. H. W. Schumacher

Tag der mündlichen Prüfung: 17. April 2012 




\section{Introduction}

The space charge region in combination with the band edge profile is fundamental for the understanding of semiconductor physics. Scanning tunneling spectroscopy is a useful tool to investigate the band gap of a bulk semiconductor and the spatially resolved band edge profile of semiconductor interfaces on the nanometer scale. Space charge regions, which are formed at the interface of different semiconductor materials or at the tip-vacuum-semiconductor interface, can be extracted from spectroscopy measurements. The space charge region of biased heterostructures can be investigated, by introducing two contacts to the sample and applying an additional potential.

For large scale investigation the space charge region in semiconductors is described by charges (electrons, holes and ionized dopant atoms) which are distributed homogeneously and averaged. The Poisson equation, which connects the charge with the potential, has to be solved for the specific geometric problem. In the rigid band model this external potential simply shifts the bands of the semiconductor, which means that the solution of the Poisson equation directly gives the band bending of the semiconductor. For investigations on the nanometer scale, the doping cannot be described by a homogeneous and averaged model anymore. This concept has already been discussed for semiconductor devices in the 1970s by Keyes and Hoeneisen [1, 2]. As an example Figure I.1 shows a simulation of a $30 \times 30 \mathrm{~nm}$ field effect transistor domain by Roy and Asenov in 2005 [3]. The impact of the random distribution of the dopants, as well as their discrete nature are seen in the fluctuations of the potential landscape, which is color coded. The influence of the random distribution has also been shown in experiment, as the controlled positioning of dopant atoms in semiconductor devices reduces fluctuations of the device characteristic [4].

A scanning tunneling microscope (STM) tip forms a space charge region in a semiconductor similar to the gate in a field effect transistor. As the STM tip has a small size the space charge region has an extension on the nanometer scale and contains only a few charged donor atoms. The rigid band model is only applicable if the external potential changes smoothly and averaged in an area larger than the crystal potential, as the concept of band structure is only valid for a periodic potential. Thus the description of a simple shift of the bands due to the potential of the donors has reached its limit on that scale. For an exact treatment one would have to solve the Schrödinger equation of the Coulomb potential of the donor cores superimposed on the potential of the crystal atoms. The surface as well as the potential of the tip would 


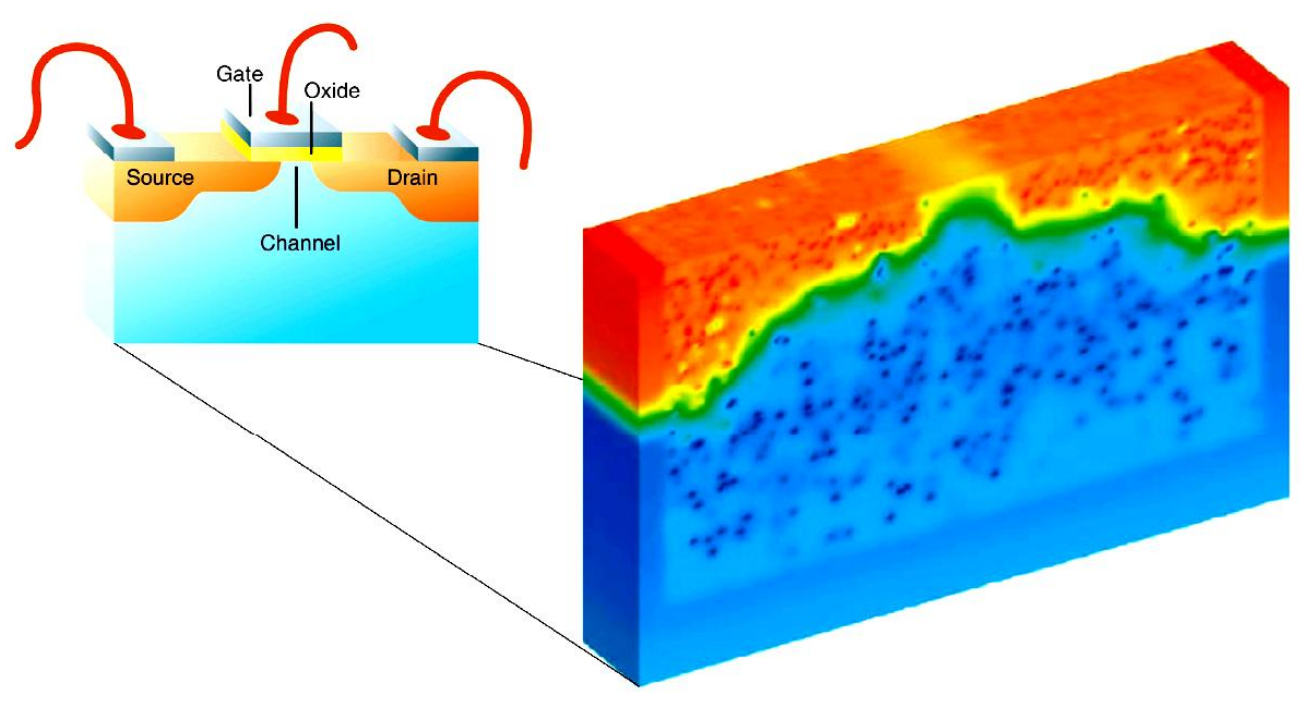

Figure I.1: Simulation of a 30x30nm field effect transistor domain. The discrete and random distributed charges of the dopant atoms are clearly visible in the color coded potential landscape. The potential fluctuations due to the random dopant distribution are responsible for the different characteristics of each device. Image from [3]

have to be included and the eigenenergies and charge density of the electrons would have to be calculated. Up to now this is far too much input for such a simulation. A more simple description, where a single donor is treated in the hydrogen model superimposed on a homogenous averaged background potential will be used in this thesis.

The investigation of the space charge region of semiconductors is done by a bottom up approach from the ionization of single donors in a bulk semiconductor to the investigations of transport phenomena in a biased heterostructure sample. The bulk semiconductor is represented by $\mathrm{Si}$ doped $\mathrm{GaAs}$, the heterostructure sample is a resonant tunneling diode consisting of two AlAs barrier separated by InAs quantum dots embedded in GaAs.

Chapter 1 gives a short overview of the fundamental theory of the STM as well as an overview of III-V semiconductors. The experimental setup and the sample preparation are presented in chapter 2 .

In chapter 3 the built up process of the space charge region caused by the STM tip is investigated using $\mathrm{Si}$ doped GaAs. The charging and discharging process of single donor atoms is resolved on the nanometer scale. The STM tip and two donors in the space charge region are sketched in the upper image of Figure I.2. In the lower image of Figure I.2 a constant current topography image of two Si donors in GaAs is shown. The disk of enhanced topographic height is caused by the charging process. For a detailed description of the charging process, the simulation of the space charge region according to Feenstra [6], which uses a homogeneous background charge, is combined with the Coulomb potential of a single donor. The advantage of the STM technique is the knowledge of the position of the donor in real space, as well as the charge state of this donor as a function of applied voltage. The model for a single donor is adopted to 

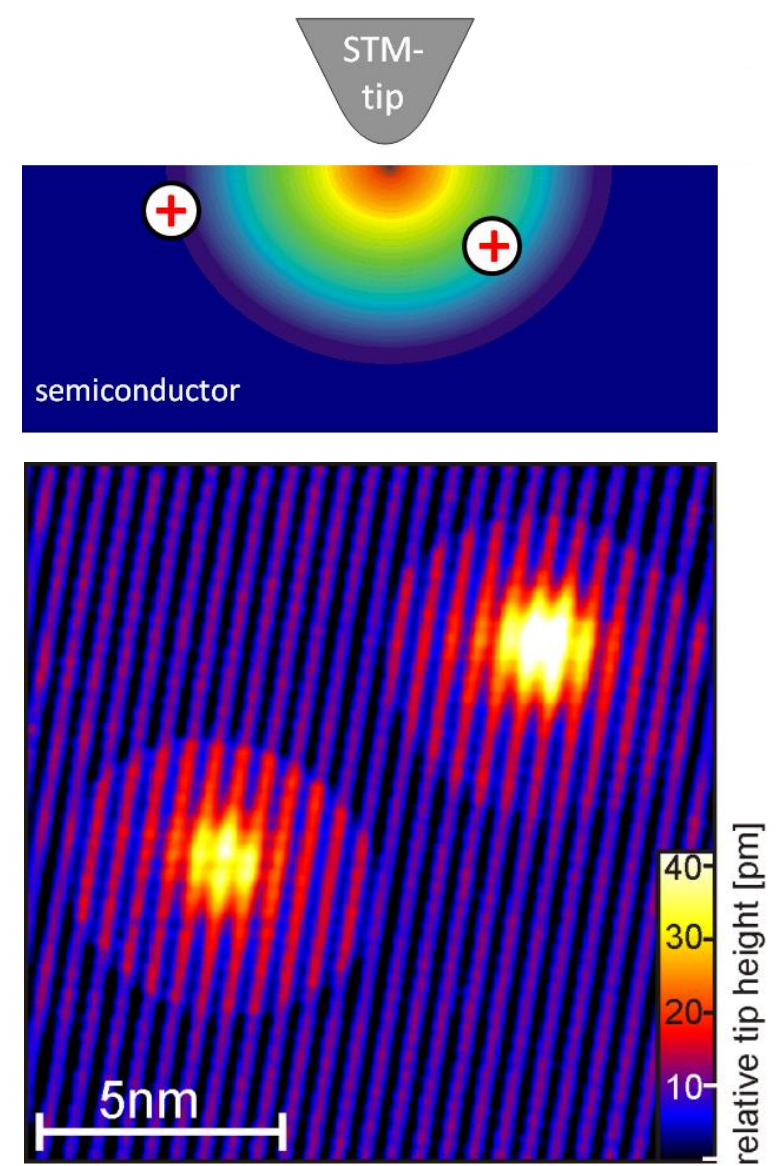

Figure I.2: The upper image sketches two donors in GaAs and the STM tip. The colored area indicates the space charge region. The lower image shows a constant current topography of two donors. The charge switching is visible by the disk-shape of enhanced topographic height.

describe the mutual interaction of double donor systems by a linear superposition. Donors which are separated only by a few nanometers show a complex ionization scenario in the space charge region. If the tip voltage and position is adjusted such that two donors change their charge states at the same applied tip voltage, it will result in a bistable behavior of the ionization.

A semiconductor heterostructure is investigated in chapter 4-7. Figure I.3 shows an atomically resolved topography image of the heterostructure. The heterostructure can be seen as the vertical enhanced plane in the image.

In chapter 4 the potential of the tip is used to modify the space charge region of the heterostructure. This allows resolving resonant tunneling phenomena, which have been observed for that specific sample in $I(V)$-transport measurements. They are located spatially on the AlAs layer and they are explained by resonant $\Gamma$-X-valley tunneling from the $\Gamma$-band of GaAs to the $\mathrm{X}$-band of the AlAs into the tip.

In chapter 5 wave functions of quantum dots which are embedded inside the heterostructure are investigated. Their behavior is compared to the one dimensional quantum mechanical harmonic oscillator problem. 


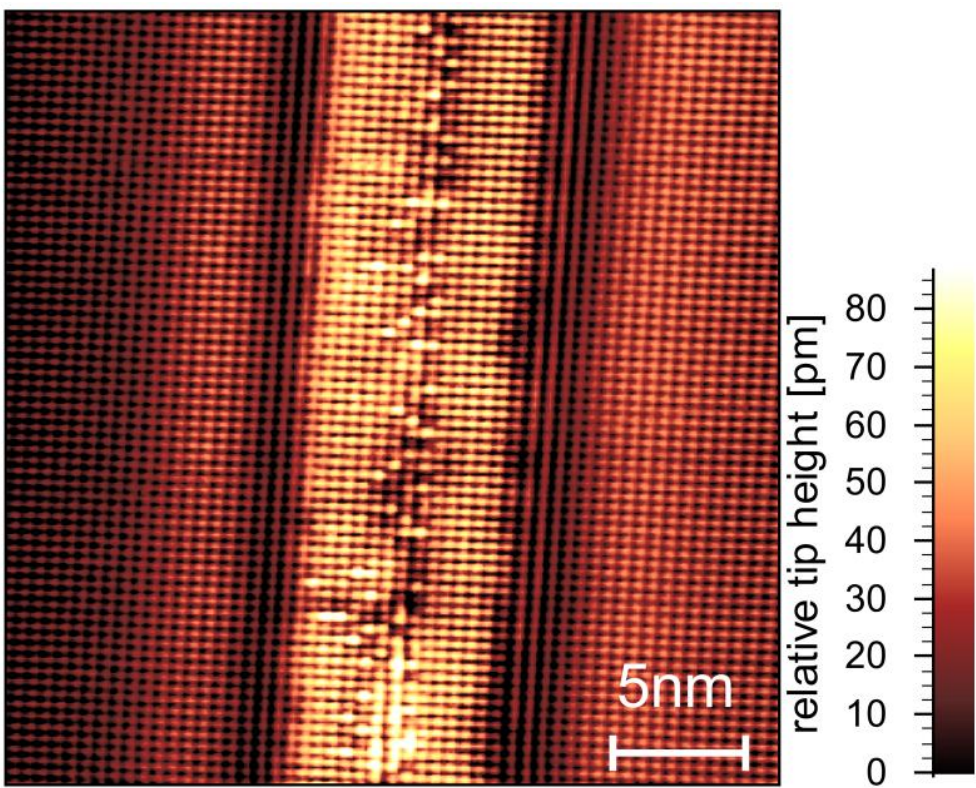

Figure 1.3: A constant current topography of the investigated heterostructure sample is shown. The AlAs layer is visible as the vertical enhancement in the middle of the image.

Chapter 6 and chapter 7 describe the extraction of the potential landscape of the heterostructure. The STM setup allows applying a lateral voltage across the heterostructure in addition to the usual tip-sample voltage. The applied lateral voltage causes a change of the space charge region at the semiconductor interface. Together with the results obtained from the bulk GaAs, the spectroscopy measurements can be used for the extraction of the potential landscape inside the structure. It is described in chapter 6 by using the onset value of the conduction band and in chapter 7 by using the state, which is formed inside the tip induced quantum dot.

A detailed description of relevant facts can be found in the Appendix:

In Appendix A.1 the difficulty of the voltage dependent position of the flat band condition at the semiconductor tip interface is discussed. This special voltage is initially not known, and still under discussion. Its knowledge is important and frequently used in this thesis. A detailed investigation of a spectroscopy data set as well as simulations of the apparent barrier height should support the used value.

In Appendix A.2 the potential landscape of the used resonant tunneling diode is simulated by solving the Poisson equation in three dimensions, including the tipsample geometry. The result is compared to the measurement and supports the interpretation of the thesis.

In Appendix A.3 the used heterostructure samples are summarized and compared with each other. 


\section{Table of Contents}

Introduction

Table of Contents $\quad$ v

1. Theoretical background 1

1.1. Theory of the scanning tunneling microscope 1

1.1.1. Standard tunnel theory 2

1.1.2. Tip induced band bending 4

1.2. Physical properties of III-V semiconductors 7

1.2.1. GaAs, AlAs and InAs bulk properties $\quad 7$

1.2.2. Crystal structure and surface states 9

2. Experiment 13

2.1. Experimental setup 13

2.2. Method of measurement 13

2.2.1. Quasi-simultaneously measured topography images 14

2.2.2. Spectroscopy and graphical visualization 14

2.2.3. Apparent barrier height 15

$\begin{array}{ll}\text { 2.2.4. Topography normalization } & 16\end{array}$

2.2.5. Lateral voltage 16

2.2.6. STM and light 17

$\begin{array}{ll}\text { 2.3. Tip preparation } & 17\end{array}$

$\begin{array}{ll}\text { 2.4. Sample preparation } & 17\end{array}$ 
$\begin{array}{ll}\text { 2.4.1. Sample orientation } & 17\end{array}$

2.4.2. Sample thinning 18

2.4.3. Ohmic contacts 18

$\begin{array}{ll}\text { 2.4.4. Initial Scratch and Sample cleavage } & 19\end{array}$

3. Charge switching of Si-donors in GaAs 21

3.1. Overview 21

3.1.1. Description of shallow donors in bulk material 22

3.1.2. Si doped GaAs investigated in STM 22

3.2. Principle of donor charge switching 24

3.2.1. From TIBB to donor binding energy 29

3.3. Extracting the Coulomb potential 30

3.4. Enhancement of the binding energy 33

3.5. Interacting donors 37

3.5.1. Description of the interaction 37

3.5.2. Principle of bistable charge switching 41

3.5.3. Resolving bistable charge switching 46

3.5.4. Analysis of error for the simulation 50

3.6. Beyond the model 52

4. Spatially resolved $\Gamma$-X-valley tunneling in an AIAs barrier

4.1. Motivation 53

4.1.1. The GaAs/AlAs heterostructure sample 54

4.2. Direct tunneling from the X-band of AIAs 57

4.3. Resonant $\Gamma$-X-valley tunneling from GaAs to AIAs 60

4.3.1. Model for tip induced resonant $\Gamma$-X-valley tunneling 60

4.3.2. The influence of the lateral voltage on the $\Gamma$-X-valley tunneling 61

4.3.3. Directional dependence of the $\Gamma$-X-valley tunneling 64

5. Quantum dot states inside a resonant tunneling diode $\quad 67$

$\begin{array}{ll}\text { 5.1. Introduction } & 67\end{array}$

5.1.1. The resonant tunneling heterostructure 68 
5.2. The quantum dot states compared to the harmonic oscillator

5.2.1. Outlook: The influence of a lateral voltage on the quantum dot state

6. Measuring the potential landscape of a biased resonant tunneling diode

6.1. Extracting the potential landscape inside a biased resonant tunneling diode

6.1.1. Extracting the potential from spectroscopy measurement

6.1.2. The potential landscape in a biased RTD

6.2. Direct measuring of the band edge profile by optical excitation

6.2.1. Motivation

6.2.2. Topographical investigation

6.2.3. Band edge profile under laser radiation

6.2.4. Outlook: The photo induced current

7. Tip induced states in low doped GaAs 91

7.1. Motivation 91

7.1.1. Verification of the tip induced state 93

7.2. Potential landscape of the tip induced state 94

7.3. The influence of the lateral voltage on the tip induced state 95

Summary 99

$\begin{array}{ll}\text { A Appendix } & 103\end{array}$

A.1 Flat band condition 103

A.1.1 Determining the flat band condition from barrier height measurement 103

A.1.2 Determination of the flat band condition from spectroscopy 108

A.1.3 Limitation of the apparent barrier height measurement 110

A.1.4 Laterally resolved apparent barrier height 111

A.1.5 Calculation of the Barrier height 112

A.2 Simulation of the heterostructure 114

A.2.1 The influence of the tip induced band bending 120

A.3 Comparison of the heterostructure samples 125 
B Bibliography and Abbreviations 131

$\begin{array}{lll}\text { B.1 Bibliography } & 131\end{array}$

$\begin{array}{ll}\text { B.2 Abbreviations: } & 137\end{array}$

$\begin{array}{ll}\text { Danksagung } & 139\end{array}$

$\begin{array}{ll}\text { Lebenslauf } & 141\end{array}$ 


\title{
1. Theoretical background
}

\begin{abstract}
The task of this thesis is the atomically resolved investigation of the ionization of single donors and the study of the built-up process of space charge regions in a homogeneous semiconductor and a semiconductor heterostructure. The experimental measurement instrumentation is the scanning tunneling microscope (STM). The theory of the imaging mechanism of the STM and the physical properties of the used samples is important for the interpretation. It is briefly introduced in this chapter.
\end{abstract}

\subsection{Theory of the scanning tunneling microscope}

The basic physical concept of a scanning tunneling microscope (STM) is the quantum mechanical tunnel effect. This effect will cause a current to flow between a metal tip and a conductive sample, if these are brought close together (a few Angstrom) and a voltage is applied simultaneously. The amount of tunnel current is exponentially dependent on the distance between the tip and the sample and can therefore be used for the distance feedback control. A voltage is applied between sample and tip. The tip is scanned across the surface, and by choosing a fixed voltage and a current setpoint, an image of the height profile of the sample is recorded.

The knowledge of the interaction between the tip and the sample is crucial for the interpretation of the obtained data. For a real tip-sample system the description is still a challenging problem. However, the usual description of the tunnel process in an STM is a first step how to interpret the obtained STM data; it will be introduced in the following subsection 1.1.1. The theory of electron tunneling between two metals was 1961 developed by Bardeen [7]. After the invention of the scanning tunneling microscopy in 1981 by Binnig and Rohrer [8], the general description of tunnel current was specialized for the STM geometry by Tersoff and Hamann [9, 10]. The obtained results from that theory were improved by Hamers [11]. For the special case of semiconductor metal tunneling the influence of the potential of the tip on the semiconductor has to be considered, which will be described in subsection 1.1.2. 


\subsubsection{Standard tunnel theory}

In 1961, Bardeen developed a theory to describe tunneling between two metals, which are separated by a barrier [7]. The basic idea of this theory is to calculate the transfer matrix element for tunneling from one state into another by a perturbation approach. The theory was originally not developed for a point contact, like the contact of the tip and sample of the tunneling microscope, but for two planar electrodes. The theory can be modified for that specific problem. A detailed derivation can be found in [12]

In this approach the tip and the sample are considered as two different subsystems, which are separated by a separation surface $(S)$. The subsystems can be described by a potential landscape for the tip $\left(U_{T}\right)$ and for the sample $\left(U_{S}\right)$. Each potential is zero in the area of the other subsystem. The many particle wave functions for the subsystems are assumed to be known. The wave functions for the tip are $\psi_{\mu}$ with the eigenenergies $E_{\mu}$ and the wave functions of the sample are $\chi_{v}$ with the eigenenergies $E_{v}$. To describe the wave function of the whole system, the quantum mechanical perturbation approach is used. The influence of one subsystem to the other is considered to be the perturbation. The wave functions of the combined system are called $\Psi$ they can be calculated by the time dependent Schrödinger equation.

$$
i \hbar \frac{\partial \Psi}{\partial t}=\left(T+U_{S}+U_{T}\right) \Psi
$$

Here $T$ is the kinetic energy. The time dependent solution is formed by a linear combination of the unperturbated wave function $\chi_{\nu}$ and $\psi_{\mu}$. Using Fermis golden rule, one obtains that the transition probability $w$ of an electron from $\chi_{\nu}$ to $\psi_{\mu}$ is proportional to the square of the matrix element $M_{\mu v}$.

$$
w_{\mu \nu}=\frac{2 \pi}{\hbar}\left|M_{\mu \nu}\right|^{2} \delta\left(E_{\nu}-E_{\mu}\right)
$$

Here the matrix element is:

$$
M_{\mu \nu}=-\frac{\hbar^{2}}{2 m} \int_{S}\left(\chi_{\nu}^{*} \vec{\nabla} \psi_{\mu}-\psi_{\mu} \vec{\nabla} \chi_{\nu}^{*}\right) d \vec{S}
$$

For the calculation of the tunnel current, it is necessary to sum up the tunneling probability $w$ from all states of the sample into all states of the tip. This can be described by the following equation:

$$
I=\frac{2 \pi e}{\hbar} \sum_{\mu \nu} f\left(E_{\mu}\right)\left[1-f\left(E_{\nu}+e V\right)\right]\left|M_{\mu \nu}\right|^{2} \delta\left(E_{\mu}-E_{\nu}\right)
$$

The first part $f\left(E_{\mu}\right) \cdot\left[1-f\left(E_{V}-e V\right)\right]$ describes that tunneling is only possible from an occupied state into an empty state. Therefore the state in the sample has to be occupied, which is described by the Fermi function $f\left(E_{\mu}\right)$. The state in the tip has to be empty which can also be described by the Fermi energy by $\left[1-f\left(E_{V}-e V\right)\right]$. In this part the voltage $V$ is applied to the sample, which shifts the occupation to higher energies. The product gives the probability for both conditions at the same time. As described above, the square of the matrix element is proportional to the transition probability of an electron from $\chi_{\nu}$ to $\psi_{\mu}$. Because of Fermis golden rule, tunneling is only possible for states with the same energy, which is expressed by the Dirac delta function. 


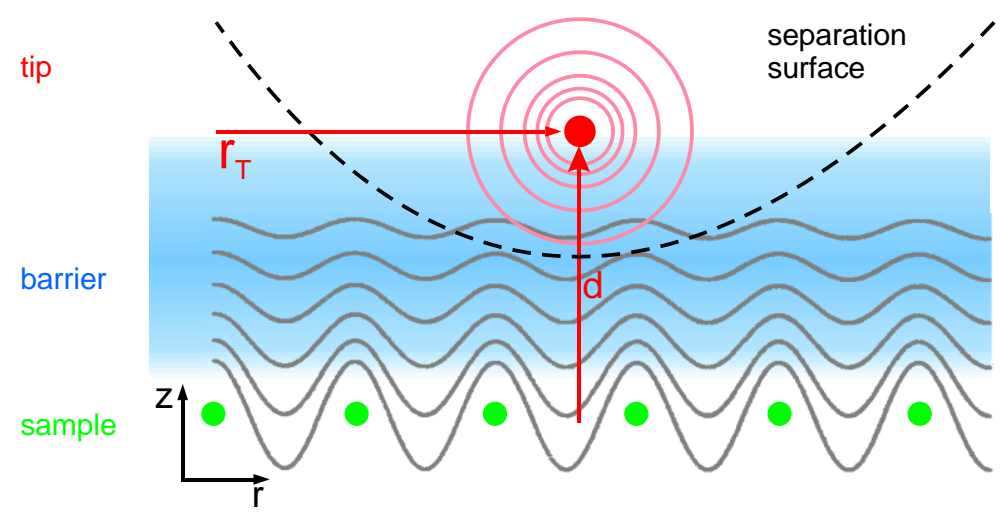

Figure 1.1 The tip-sample geometry is shown schematically. The last tip atom is marked by a red circle and the atoms of the sample by green circles. The grey and red lines represent lines of constant density of states of the sample and the tip, respectively. The tips last atom is located at the position $r_{T}$ and is described by an $\mathrm{s}-$ like wave function. (Figure from [13])

Tersoff and Hamann used this result, obtained from the theory of Bardeen, and modified it for the special geometry of a probe tip in front of a sample [10]. Usually, the shape and electronic structure of the tip is not known in experiment, in the model it is described by a spherical potential well. By using this approximation, it is possible to receive a description of the electronic structure of the sample.

The starting point of the Tersoff and Hamann model is the equation of the tunnel current (see equation (1.4)). Two basic assumptions are made for simplifying the equation of the tunnel current: An STM is usually working at room temperature or lower temperature. The broadening of the Fermi function is therefore smaller than $25 \mathrm{meV}$. The second assumption is that the voltage applied between tip and sample is in the order of $10 \mathrm{mV}$. This assumption is suitable for metal samples, where small voltages are usually used in experiment. At this point it should be mentioned that this work is a study of semiconductor samples, which are usually investigated at higher voltage due to their large band gap. This will be considered later.

Using those two approximations one can simplify the product of the two Fermi functions by the Dirac delta function multiplied with the voltage $\mathrm{e} V$.

$$
I=\frac{2 \pi}{\hbar} e^{2} V \sum_{\mu \nu}\left|M_{\mu \nu}\right|^{2} \delta\left(E_{\nu}-E_{F}\right) \delta\left(E_{\mu}-E_{F}\right)
$$

As a next step it is necessary to calculate the matrix element, using equation (1.3). The wave functions of the tip are approximated by an asymptotic spherical symmetric s-like wave function. This is motivated by the idea that at the smallest distance between tip and sample, the apex of the tip consists only of a few atoms and the tip apex can be described as a round sphere. In Figure 1.1 the tip-sample geometry is shown schematically.

Tersoff and Hamann describe the wave function in the sample with wave functions, which are Bloch-like parallel to the surface, and which decay exponentially into the vacuum perpendicular to the surface. With these two descriptions it is possible to calculate the matrix element. It is proportional to the probability density of the sample states at the position of the tip $\mathrm{r}_{\mathrm{T}}$. The tunnel current can be expressed as: 


$$
I_{T} \propto e V \rho_{T}\left(E_{F}\right) \sum_{\mu}\left|\psi_{\mu}\left(\vec{r}_{T}\right)\right|^{2} \delta\left(E_{\mu}-E_{F}\right)
$$

This means that the tunnel current is proportional to the density of states of the tip at the Fermi energy $\rho_{T}\left(E_{F}\right)$, as well as to the sample voltage $V$. The magnitude of the sum is the local density of states (LDOS) of the sample surface at the Fermi energy.

$$
\operatorname{LDOS}\left(\vec{r}_{T}, E_{F}\right)=\sum_{\mu}\left|\psi_{\mu}\left(\vec{r}_{T}\right)\right|^{2} \delta\left(E_{\mu}-E_{F}\right)
$$

As the tunnel current is proportional to the LDOS of the sample, a constant current topography image is a surface of constant LDOS.

$$
I \propto \operatorname{LDOS}\left(\vec{r}_{T}, E_{F}\right)
$$

By the calculation of the tunnel current, the most crucial approximation is that the tip is only described by an s-like wave function. Applying this to a semiconductor surface, one obtains that the expected resolution is less than the atomic lattice constant [9], which is in contradiction to experimental observation. Using p- and d-like wave functions for the description of the sample, the predicted resolution is closer to reality. The calculated matrix elements for $\mathrm{p}$ - and d-like tip wave functions can be found in [12].

The assumption for small voltages is not valid for semiconductors, due to their large band gap. For higher applied voltages, the tunnel current is no longer proportional only to the sample LDOS at the Fermi energy. Hamers introduced an energy dependent transmission probability $T(E, e V, d)$ to describe the transmission of states at different energies than the Fermi energy [11]. The tunnel current is obtained by the integration over all energies where tunneling can occur.

$$
I=\int_{E_{F}}^{E_{F}+e V} \rho_{T}(E-e V) \operatorname{LDOS}\left(r_{T}, E\right) T\left(E, e V, r_{T}\right) d E
$$

The transmission probability is calculated by the WKB approximation. This includes the change of the barrier due to the applied voltage and the specific energy of each tunneling electron. The vacuum barrier is described by the sample and tip work functions $\chi$ and $\phi_{\mathrm{T}}$, as well as by the distance between the tip and the surface $d$.

$$
T(E, e V, d)=\exp \left(-\frac{2 d \sqrt{2 m}}{\hbar} \sqrt{\frac{\chi+\phi_{T}}{2}+\frac{e V}{2}-E}\right)
$$

\subsubsection{Tip induced band bending}

The tip-sample system used in the STM is in principle comparable to a Schottky contact or to the gate of a field effect transistor. A voltage $\left(\mathrm{V}=\phi_{\text {sample }}-\phi_{\text {tip }}\right)$ is applied where the potential of the metal tip is $\phi_{\text {tip }}$ and the potential of the sample back contact is $\phi_{\text {sample }}$. The potential of the tip introduces a potential a few ten nanometer into the semiconductor sample. The In the rigid band model the external potential just shifts the energetic position of the bands of the semiconductor linearly without changing the band structure. For the case of the STM sample, this is called tip induced band 


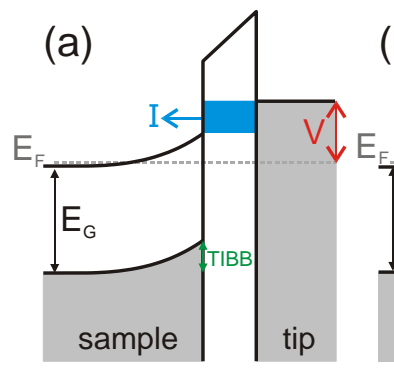

(b)

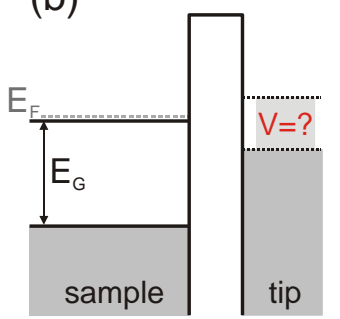

(c)

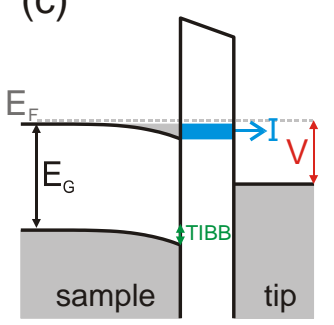

(d)

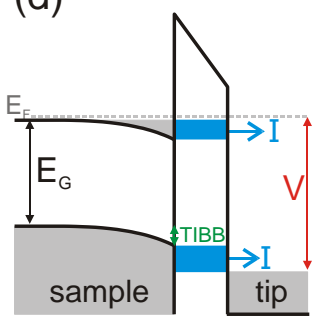

Figure 1.2: Schematic energy band diagram for the tip-sample system at different applied voltages $\left(\mathrm{V}=\phi_{\text {sample }}-\phi_{\text {tip }}\right)$. Image (a) illustrates the tunneling condition for positive voltages. Image (b) is the special case when the bands in the semiconductor are flat. Its voltage is not initially known and has to be evaluated from measuring the apparent barrier height. (c) Small negative voltages and (d) large negative voltages.

bending (TIBB) and was first introduced by Feenstra and Stroscio [5]. The amount and the extension of the TIBB are dependent on the amount of additional charge inside the semiconductor. Depending on the applied voltage $\mathrm{V}=\phi_{\text {sample }}-\phi_{\text {tip }}$ the additional charge are ionized donors or free charge carriers for n-doped material ${ }^{1}$. In metals the screening length is usually very short $<1 \AA$, as they have a high density of states at the Fermi-energy ${ }^{2}$. In contrast, semiconductors have a band gap, and if the screening is done by ionized donors, the screening length is determined by the doping concentration. It is typically in the order of a few ten nanometers for normally doped samples $\left(\sim 10^{17}-10^{18} \mathrm{~cm}^{-3}\right)$ [14]. The GaAs $\{110\}$ surfaces, which are investigated in this thesis, have no surface states within the band gap. The surface resonances lie energetically in the conduction or valence band [15]. Thus, within the band gap no additional states are available for the screening, which is the reason that the amount of TIBB is different for different applied voltages. The interpretation of the tunneling spectra and their features is thus more complicated, as the relation between applied voltage and the energetic position inside the semiconductor is not linear anymore [16].

In Figure 1.2 the energy band diagram for the tip-sample system perpendicular to the surface is shown for different voltages. In Figure 1.2(a) the situation is illustrated for large positive voltages $\left(\mathrm{V}=\phi_{\text {sample }}-\phi_{\text {tip }}\right)$. The bands in the semiconductor are lifted upwards, the screening of the potential induced by the tip is done by the ionization of donors and the tunnel current can flow from the filled states of the tip to the empty states in the conduction band of the semiconductor. Figure 1.2(b) shows the special condition when the voltage of the tip is such that the bands in the semiconductor are flat. For the simulation of the voltage dependent $\operatorname{TIBB}(V)$ the knowledge of the special voltage of the flat band condition is important, as a shift of the flat band condition would shift the curve of the voltage dependent $\operatorname{TIBB}(V)$. The voltage for the flat band condition is initially not known. It has to be determined for example by the measurement of the apparent barrier height and by comparing features in the spectroscopy measurements (see section A.1). Figure 1.2(c) visualizes the energy band diagram for small negative voltages $\left(\mathrm{V}=\phi_{\text {sample }}-\phi_{\text {tip }}\right)$. The bands of the semiconductor are shifted below the Fermienergy $\left(\mathrm{E}_{\mathrm{F}}\right)$ and the screening is done by the electrons

\footnotetext{
${ }^{1}$ As for this thesis only n-doped material is used, the discussion is reduced to donors. It would be similar for p-doped material and acceptors.

2 This is why the screening in the tip can be neglected.
} 


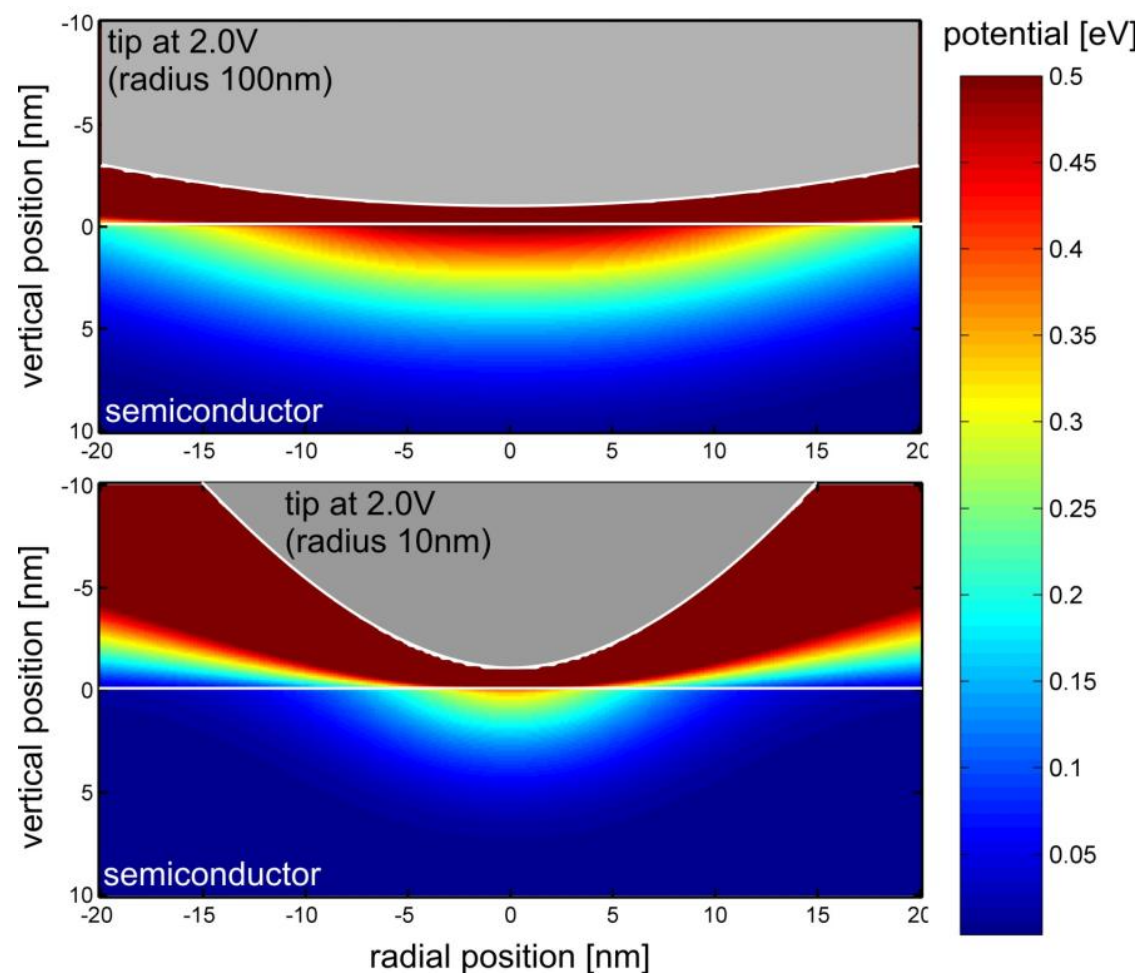

Figure 1.3: Comparison of the spatial extension of the TIBB for two different tip (grey) geometries. The potential is color-coded. The extension as well as the amount of the TIBB is much larger for a very blunt tip with a radius of $100 \mathrm{~nm}$ compared to a sharper tip with a radius of $10 \mathrm{~nm}$. The voltage of the tip is in both cases assumed to be $2 \mathrm{~V}$. (Solver from [20], Image from [13])

which are accumulated in the so called tip induced quantum dot $[17,18,19]$. Electrons in that quantum dot can tunnel into empty states of the tip, which leads to a tunnel current in an energy region where no current is expected, as it is the energy of the band gap of the semiconductor. Figure 1.2(d) illustrates the situation for large negative voltages $\left(\mathrm{V}=\phi_{\text {sample }}-\phi_{\text {tip }}\right)$. The tunnel current can flow from the still existing tip induced quantum dot, as well as from the filled states in the valence band. These four figures sketch that the energy scale in the semiconductor is not related linearly to the applied voltage. For the interpretation of the data it is therefore important to know the voltage dependent $\operatorname{TIBB}(V)$.

Additional states than expected from the band structure, like the tip induced states can occur in the expected band gap (Figure 1.2(c)). This is an indication that the simple rigid band model is not good in that case, but by allowing states to form in the potential it is still a useful description.

For the simulation of the voltage dependent $\operatorname{TIBB}(V)$ the Poisson equation has to be solved for the tip-sample geometry. In the beginning of the STM theory the problem was usually solved in one dimension. Using this simplification, one has to assume that the tip is extended infinitely above the sample. As the tip geometry differs strongly from a planar plate, and is in contrast very sharp with an apex radius smaller than $10 \mathrm{~nm}$ (see section 2.3), the problem should be solved in three dimensions. This has been shown recently by Feenstra [6], by comparing the one dimensional solution of the TIBB with a more realistic model of hyperbolic tip geometry in front of the 
semiconductor. The amount of the potential induced in the semiconductor changes by up to a factor of 4 , depending on the size of the tip. The comparison of the lateral dependence of the $\operatorname{TIBB}(x, y)$ for two tip geometries is shown in Figure 1.3; the solver provided by Feenstra was used for the calculations [20]. It can be seen that for a tip with a large radius $(100 \mathrm{~nm})$ the spatial extension of the TIBB is larger, and the amount of TIBB is higher, compared to a tip with a smaller radius $(10 \mathrm{~nm})$. In addition to the parameters of the tips geometry, the parameters which have to be included in a simulation of the TIBB are the material parameters of the semiconductor. This is the dielectric constant $\varepsilon_{\mathrm{r}}$, the doping concentration, which is included homogeneously and determines the averaged amount of ionized donors per cubic centimeter. The binding energy of the donors, leads to the external potential where the charge is available. The effective masses of the conduction and the valence band determine the potential dependent amount of negative charge per cubic centimeter.

This is discussed in more detail in Appendix A.2, using a different solver to solve the Poisson equation and receiving the TIBB for the geometry of a GaAs-AlAsheterostructure.

\subsection{Physical properties of III-V semiconductors}

In this section, the physical properties of the investigated materials, which are important for the interpretation of the data, are discussed. Two different samples were investigated. The first sample is $\mathrm{Si}$ doped $\mathrm{GaAs}$, the second sample consist of a heterostructure of GaAs, AlAs and InAs. For the interpretation of the STM data, the knowledge of the electronic properties (subsection 1.2.1), the crystal structure and the surface states (subsection 1.2.2) is important.

\subsubsection{GaAs, AIAs and InAs bulk properties}

A homogeneous GaAs sample and a heterostructure consisting of GaAs layers, AlAs layers and InAs quantum dots, is investigated. Therefore a short summary of the bulk properties of these materials will be given here. In Figure 1.4 the band structure of GaAs, AlAs and InAs is shown. The smallest band gap is indicated in red. GaAs and InAs have a direct band gap, whereas AlAs has an indirect band gap with the conduction band minimum close to the X-point. In Table 1, the band gaps at the $\Gamma$-point and the $\mathrm{X}$-point for the three different materials are summarized.

The knowledge of the electronic structure of the semiconductor-semiconductor interface is important for the interpretation of the STM spectroscopic data. In particular, the question how the two band schemes align with each other and where the band gap will be formed energetically is crucial. The parameter describing this is the band offset $\Delta \mathrm{E}_{\mathrm{V}}$, which is the relative offset of the two valence band maxima, or equivalently the offset of the two conduction band minima $\Delta \mathrm{E}_{\mathrm{C}}$. One possibility to describe the band offset is the electron-affinity rule, which was developed by Anderson in 1962 [24]. The band offset is calculated by the difference between the electron affinities of the two semiconductors. For GaAs the electron-affinity is $4.07 \mathrm{eV}$ for AlAs it is $3.5 \mathrm{eV}$ [25]. Using the simple model one would expect a band offset of $\Delta \mathrm{E}_{\mathrm{C}}=0.57 \mathrm{eV}$. 

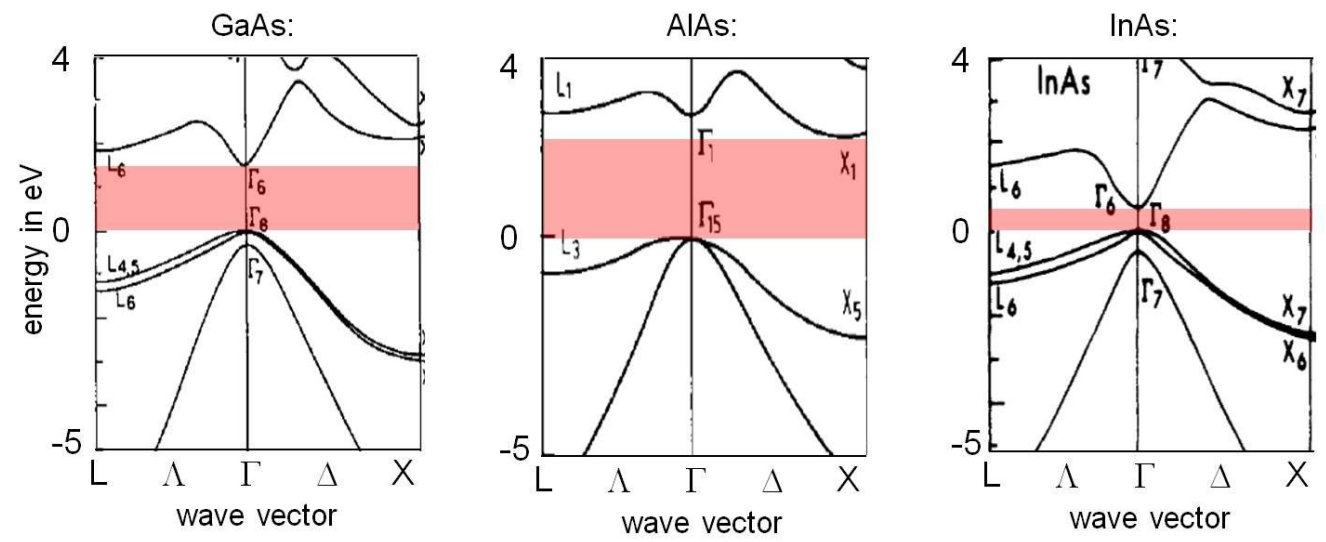

Figure 1.4: Band structure of GaAs [21], AlAs [22] and InAs [21]. The band gap is indicated in red. It can be seen that GaAs and InAs have a direct band gap, whereas AlAs has an indirect band gap.

\begin{tabular}{l|lll}
\hline \hline & GaAs & AlAs & InAs \\
\hline $\mathrm{E}_{\mathrm{g}}(\Gamma)(\mathrm{eV})$ & 1.519 & 3.099 & 0.417 \\
$\mathrm{E}_{\mathrm{g}}(\mathrm{X})(\mathrm{eV})$ & 1.981 & 2.24 & 1.433 \\
\hline \hline
\end{tabular}

Table 1: Bulk band gaps for the three materials at $5 \mathrm{~K}$ [23].

For the GaAs / AlAs interface the band offset has been under intensive discussion for a long time. The split between the conduction and the valence band has been established to be 65:35 and, confirmed by various measurement methods, the valence band offset is: $\Delta \mathrm{E}_{\mathrm{V}}=0.53 \mathrm{eV}$ [23]. With the knowledge of the band gaps (see Table 1) this results in a conduction band offset of $\Delta \mathrm{E}_{\mathrm{C}}=1.05 \mathrm{eV}$ for the $\Gamma$-point and $\Delta \mathrm{E}_{\mathrm{C}}=0.19 \mathrm{eV}$ for the $\mathrm{X}$-point. Both values differ from the value obtained from the simple electron-affinity rule.

To understand the deviation of the band offset extracted from the simple electron affinity model, the concept of a dipole at the ideal, defect free, semiconductorsemiconductor interface was introduced by Tersoff [26]. This concept is similar to the metal induced gap states for a semiconductor-metal interface of a Schottky contact. The dipole results from interface induced states. The origin of these states is due to the fact that the bulk electronic states of one semiconductor, which lie energetically in the band gap of the other semiconductor, decay a few Angstrom in the latter one. These states have primarily conduction band character if they lie energetically close to the conduction band or primarily valence band character if they are close to the valence band. The occupation of a gap state results in a local excess of charge in proportion to its degree of conduction band character. An empty gap state leads to a local charge deficit in proportion to its degree of valence band character. The system will tend to that amount of band offset, in which the net dipole charge is zero because this minimizes the free energy of the whole system. 


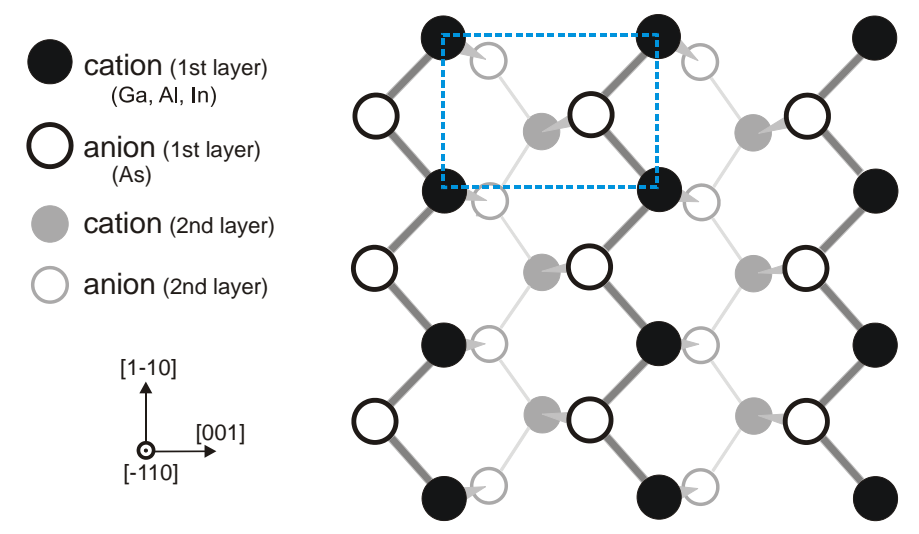

Figure 1.5: The GaAs $(-110)$ cleavage plane in top view. The two first layers are shown. The blue box shows the surface unit cell.

\subsubsection{Crystal structure and surface states}

The three materials which are used in the investigated sample, GaAs, AlAs and InAs belong to the group of III-V compound semiconductors. The group III-atoms gallium, aluminum and indium are the cations; the group $\mathrm{V}$ atom arsenic is the anion. The atoms crystallize in zincblende structure, which consists of two interpenetrating face centered cubic (fcc) sublattices. One of the sublattices is displaced from the other by $1 / 4$ along a diagonal of the unit cell. Each sublattice is occupied by either cations or by anions. The lattice constants for $\mathrm{GaAs}=0.565 \mathrm{~nm}$ for $\mathrm{AlAs}=0.566 \mathrm{~nm}$ and for InAs $=0.605 \mathrm{~nm}$ [27].

In the STM experiment, the $\{110\}$ surface planes are investigated. The heterostructure is cleaved along the (110) directions in ultra high vacuum (UHV) to obtain a clean surface. Due to the absence of neighboring atoms at the surface, the interatomic forces in the upper lattice plane are considerably changed. This causes a change in the atomic position with respect to the bulk [14]. The surface gallium-atoms are pulled towards their three neighbors and the As-surface atoms tilt up from the ideal surface plane, which is called buckling. The atomic displacement of the AlAs surface is similar to the atomic displacement of the GaAs surface [28]. Figure 1.5 shows the first two layers of the cleavage plane in top view. 


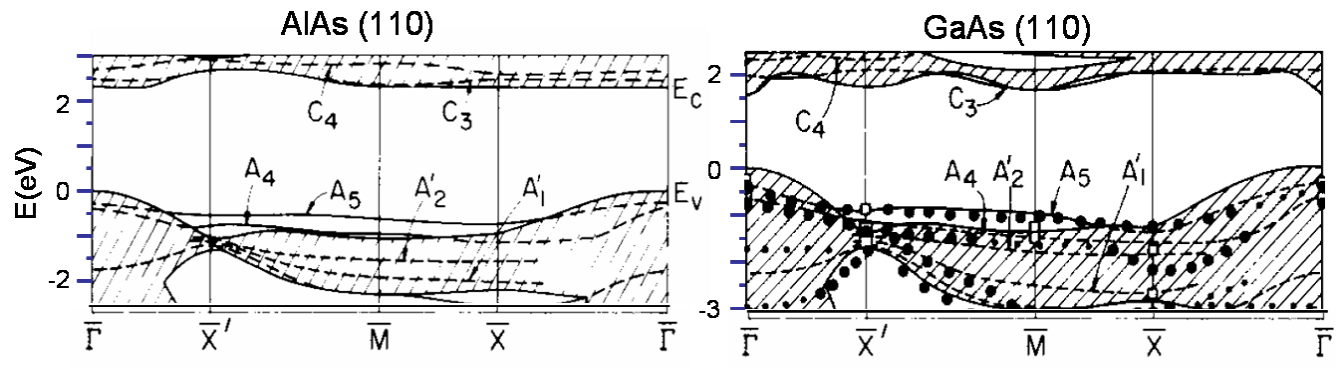

Figure 1.6: The calculated (tight-binding) surface state dispersion curve for the (110) surface of AIAs [29] and GaAs [30] are shown. The surface states do not lie inside the band gap, but in the conduction and valence band.

The relaxation of the topmost atoms causes the (110) surface to be free of surface states within the bulk band gap [14]. Instead, the surface states lie in the conduction and valence band, and are thus called surface resonances. In Figure 1.6 the calculated surface state dispersion curves are shown for AlAs(110) [29] and GaAs(110) [30]. For the bulk band structure the authors used a tight-binding method introduced by Vogel et al., which has the advantage that this method predicts also the indirect-gap bulk band structure [31]. Note that in Figure 1.6 the projected band structure in (110)direction is plotted. For the AlAs band structure the band edge at the K-point, which is the (110)-direction, is almost at the same energy as the X-point, therefore the projected band gap at the $\Gamma$-point is almost at the same energy as the projected band gap of the X-point and the indirect band gap is not easily seen for the projected band gap. The major feature of the surface state dispersion curve for the two materials is similar. Thus, the features are labeled by the same notation: $A_{i}$ for the mainly anion derived states and $\mathrm{C}_{\mathrm{i}}$ for the mainly cation derived states.

The reorganization of the topmost atoms induces also a charge transfer from the dangling bonds of the cations to the dangling bond of the anions. Thus, the dangling bonds of the anions are filled, and the dangling bonds of the cations are empty. In Figure 1.7 the calculated integral LDOS of the surface states of the $\operatorname{InP}(110)$ surface is shown. InP is also a III-V semiconductor and it has been shown that STM images on InP show no significant differences to GaAs [32]. The filled states (A4 and A5) have the largest LDOS at the position of the anions; the empty states have the largest LDOS close to the cations. By comparing topographies at different voltages a shift in the corrugation maxima is a sign that the surface state which is imaged has changed. This is especially the case if one compares images taken at large negative voltages with images taken at large positive voltages. For positive voltages the empty states are visible and the corrugation maxima should occur on the cations, for negative voltages the filled states are visible and the corrugation maxima should be shifted to the position of the anions. 


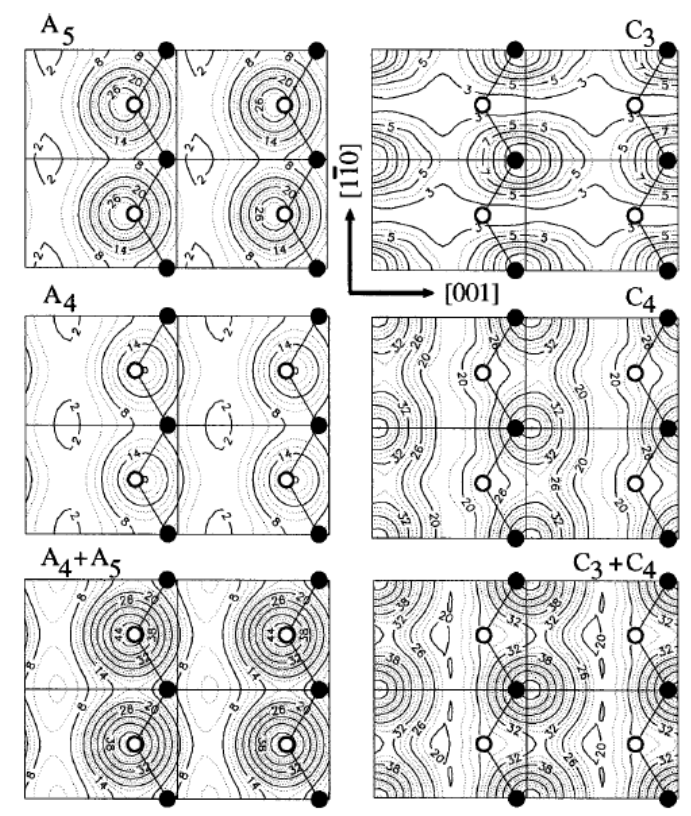

Figure 1.7: Calculated lateral distribution of the integral LDOS for the surface states of the $\ln \mathrm{P}(110)$ surface. The positions of the anions are symbolized as white circles the positions of the cations are symbolized as black circles. The filled surface states (A5 and A4) have the largest LDOS at the position of the anions; the empty surface states (C3 and C4) have the largest LDOS close to the position of the cations. Image from [32]. 



\section{Experiment}

This chapter contains a description of the specific experimental setup. As some methods of measurement are not typically used in the standard STM technique, they will be introduced here. The sample and tip preparation are presented in the second part.

\subsection{Experimental setup}

In this thesis a home built scanning tunneling microscope (STM) is used. It works under UHV conditions at a temperature of $6 \mathrm{~K}$. The scanning unit is a Besocke-typeSTM [33], which is attached to a liquid helium bath cryostat. The sample support has 6 electrically isolated contacts. The tip is grounded and the voltage is applied to the sample. The voltage between sample and tip is defined in this thesis by $\mathrm{V}=\phi_{\text {sample }}-\phi_{\text {tip. }}$. Here $\phi_{\text {sample }}$ is the potential of the back-contact of the sample and $\phi_{\text {tip }}$ is the potential of the tip. The sample holder is constructed such that two of those independent contacts are used. This allows applying a voltage across the heterostructure in addition to the usual tip-sample voltage. An optical access to the sample and the tip during measurement allows one to irradiate laser light on the sample. The laser is mounted to the stage of the UHV-chamber to avoid external oscillations on the laser beam. More information about the experimental setup can be found in $[13,34]$.

\subsection{Method of measurement}

Some special measurement methods which are not used in standard STM investigation but are important for the measurements in this thesis will be introduced shortly in the following subsections. 


\subsubsection{Quasi-simultaneously measured topography images}

It can be useful to compare the atomic corrugation of constant current topography images at different voltages. For different bias voltages different surface states are imaged. That means that the corrugation maxima can shift for example from the gallium atoms to the arsenic atoms [35]. Comparing two images which are taken directly after each other has the disadvantage that the absolute position of the STM tip can get lost due to thermal drift and the shift of the corrugation maxima cannot be seen. It can also happen that the tip will modify during the image recording process, so that the two images are not comparable any more.

To avoid these disadvantages, the topography images are taken quasi simultaneously. This means that the tip scans each line of the image a few times. For each line the bias voltage (or current setpoint) is changed. When all chosen voltages are scanned the tip starts again at the next line. Lines with the same voltage are saved as a single topography image. As the time for taking a line is much shorter than for taking a whole image, the thermal drift is not a problem anymore. If the tip modifies during the measurement, the impact on the topography is similar for all voltage, and the quasisimultaneously taken topographies are directly comparable.

\subsubsection{Spectroscopy and graphical visualization}

Another commonly used tool is the laterally resolved $I(V)$-spectroscopy. The current $I(x, y, z, V)$ is a function of the space coordinates $x, y, z$ and the voltage $V$. For a fixed voltage the tip height $z$ is adjusted for each topography point. After that the feed back loop is stopped and the $I(V)$ curve is taken. The measured data set is the current $I(x, y, V)$ as a function of the space coordinates $x, y$ and the voltage $V$. The data set is four dimensional and thus, it is not possible to show it in one graphic. The differential conductivity $\mathrm{d} I / \mathrm{d} V(x, y, V)$ is derived numerically.

The graphical visualization can be done in different manners:

- Single spectra at a certain position are shown in a usual two dimensional plot, with the current $I(V)$ or the differential conductivity $\mathrm{d} I / \mathrm{d} V(V)$ plotted as a function of voltage.

- The data along a line in the topography image are plotted in a color coded image, in this thesis this is called spectrum section. The horizontal-axis is usually the lateral displacement; the vertical-axis is the voltage. The current $I(x, V)$ or the differential conductivity $\mathrm{d} I / \mathrm{d} V(x, V)$ are color coded.

- The data at a certain voltage are plotted laterally resolved; in this thesis this is called spectrum map. The horizontal-axis as well as the vertical-axis are the lateral displacement in different directions. The current $I(x, y)$ or the differential conductivity $\mathrm{d} I / \mathrm{d} V(x, y)$ are color coded. 


\subsubsection{Apparent barrier height}

The dependence of the tip-sample distance $d$ on the tunnel current $I$ is exponential:

$$
I \propto \exp (-2 \kappa d)
$$

The decay constant $\kappa$ can be expressed by defining the apparent barrier height $\phi_{\text {app}}$, which is in principle the average of the "real" barrier seen by the tunneling electron, and with the electron mass $m$ and the Planck constant $\hbar$ :

$$
\kappa=\frac{\sqrt{2 m \phi_{a p p}}}{\hbar}
$$

According to these two relations, the apparent barrier height can be determined by measuring the current $I_{1}$ and $I_{2}$ for two different tip-sample distances $\Delta d=d_{1}-d_{2}$ :

$$
\phi_{a p p}=\frac{\hbar^{2}}{8 m}\left(\frac{\ln \left(I_{1} / I_{2}\right)}{\Delta d}\right)^{2}
$$

For the apparent barrier height in the units of electron volt and the tip-sample distance between both measurements in Angstrom, the equation for the apparent barrier height is:

$$
\phi_{\text {app }}[\mathrm{eV}]=0.95\left(\frac{\ln \left(I_{1} / I_{2}\right)}{\Delta d[\AA]}\right)^{2}
$$

The measurement of the current for two different tip-sample distances can be done in different ways:

A possibility is to measure the apparent barrier height in addition to the spectroscopy measurement. Between the topography adjustment and the recording of the $I(V)$-curve, the distance between tip and sample is alternately reduced and decreased (typically the difference is chosen to be $0.4 \AA$ ), whereas the voltage is kept constant. At both distances the current is measured and from this the apparent barrier height is calculated. At a fixed point the apparent barrier height is averaged over a few modulations. One obtains the apparent barrier height $\phi_{a b h}\left(x, y, V_{0}\right)$ as a function of the space coordinates $x, y$ and a fixed voltage $V_{0}$.

Another possibility is to measure the apparent barrier height with quasisimultaneously taken topography images. For this case the applied voltage is kept constant, and the setpoint current is varied. The relative distance between the topography images is extracted and together with the known current setpoints the apparent barrier height can be calculated.

Sometimes it is necessary to have a voltage dependent measurement of the apparent barrier height. In this thesis this was realized by measuring two $I(x, y, V)$ spectroscopy sets taken at two different current setpoints, which means two spectroscopy sets taken at two different tip-sample distances. As the feedback loop is stopped during the recording of the $I(V)$-curve, the currents for a certain voltage can be used to calculate the apparent barrier height. One obtains the apparent barrier height $\phi_{a b h}(x, y, V)$ as a function of the space coordinates $x, y$ and the voltage $V$. 


\subsubsection{Topography normalization}

In small sized topography image the atomic corrugation is usually visible. That means that the topographic height varies in lateral space $x$ and $y$. As a spectroscopy measurement is stabilized at a constant current topography image, the amount of current for two different positions is therefore not directly comparable. If the tip is close to the surface the current will be larger compared to the situation that the tip is in a larger distance to the surface. If the amount of current is necessary for the interpretation, the spectroscopy is normalized to a flat plane. The quantity $\Delta z$ is the vertical distance of the topographic height to the flat plane. The normalization avoids crosstalk from the constant current topography image. The normalization is done by simultaneously measuring the lateral resolved apparent barrier height. Using equation (2.1) the measured current $I_{0}$ at each point and voltage is normalized, the result is $I_{\text {norm }}$.

$$
I_{\text {norm }}(x, y, V)=I_{0}(x, y, V) \cdot \exp (-\kappa(x, y) \Delta z(x, y))
$$

Here $\kappa$ is according to equation (2.2), where the apparent barrier height is a function of the lateral displacement $x$ and $y$ at a constant voltage.

\subsubsection{Lateral voltage}

In addition to the usual STM voltage, which is applied between tip and sample, the STM setup used in this thesis allows applying a voltage across the sample. This is realized by using a sample holder which has two electrically isolated contacts. The sample holder is connected to the STM sample support. The two contacts can be addressed with the same voltage, which results in the usual STM mode.

It is also possible to address the sample with different voltages $V_{\text {left }}$ and $V_{\text {right }}$. Here the left and the right side correspond to the left and the right side of the shown images, the growth direction (100) is from left to right in the images. A sketch of the geometry of tip and sample and the connections is shown in Figure 2.1. The difference between the two voltages is the voltage applied across the heterostructure $V_{H e t}$ :

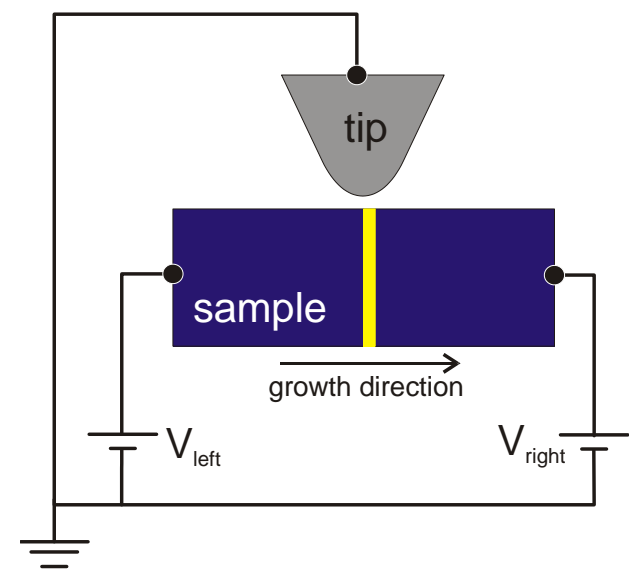

Figure 2.1: Geometry of the sample and the tip. Each of the two ohmic contacts is connected to a voltage source, which is labeled with $V_{\text {left }}$ and $V_{\text {right. }}$ The tip is grounded. 


$$
V_{\text {Het }}=V_{\text {left }}-V_{\text {right }}
$$

The usual STM voltage $V_{\text {STM }}$ is added to each contact. The offset of each contact is in the measurement adjusted in the way that the voltage for $V_{S T M}=0 \mathrm{~V}$ are symmetrically distributed. This can be expressed by the following equations:

$$
V_{\text {left }}=\frac{V_{H e t}}{2}+V_{S T M} \text { and } V_{\text {right }}=-\frac{V_{H e t}}{2}+V_{S T M}
$$

Alternatively one can adjust the voltage such that one contact is used as a reference for the usual $V_{S T M}$ voltage:

$$
V_{\text {left }}=V_{\text {Het }}+V_{S T M} \text { and } V_{\text {right }}=V_{S T M}
$$

In a spectroscopy measurement $V_{H e t}$ is kept constant, and the voltage ramp is applied simultaneously to both contacts by applying the ramp to $V_{S T M}$.

\subsubsection{STM and light}

The STM has an optical access to the sample and the tip during measurement. This allows irradiating laser light on the sample. The employed laser has a wavelength of $784 \mathrm{~nm}$, which corresponds to the energy of $1.58 \mathrm{eV}$. The band gap of GaAs at $5 \mathrm{~K}$ is $1.52 \mathrm{eV}$, thus, the light is able to excite electron hole pairs in GaAs.

\subsection{Tip preparation}

The tips which are used in the STM measurement are made from polycrystalline tungsten wire. The tungsten wire is electro-chemically etched in a $\mathrm{KOH}$ base. The etched tungsten tips are cleaned in water and ethanol and have a typical shaft length of 200-300 $\mu \mathrm{m}$. After cleaning, they are transferred into an UHV-chamber for further tip preparation. In the UHV chamber, the tips are annealed to remove dirt and the oxide layer. In a next step the tips are sputtered by Argon ions, which are accelerated with $3 \mathrm{kV}$ onto the tip. This process should sharpen the tip. The last step is a characterization step. The tips are brought in front of a metal plate and by applying a voltage between tip and metal plate a field emission current-voltage characteristic is recorded. In previous works, a detailed treatment of the tip preparation technique used in Göttingen can be found [36, 37]. In the latter quotation, it was verified by scanning electron microscopy images that this procedure leads to sharp tips with a radius of curvature of the tip apex of only a few nanometers and an opening angle of $<90^{\circ}$.

\subsection{Sample preparation}

\subsubsection{Sample orientation}

The natural cleavage planes of GaAs are the (011) and (0-11) planes. The GaAs wafer was cleaved into $6 \times 9 \mathrm{~mm}$ sized pieces using these cleavage planes. The orientation of the long sample side can be chosen to be in (011) or (0-11) direction. This is 


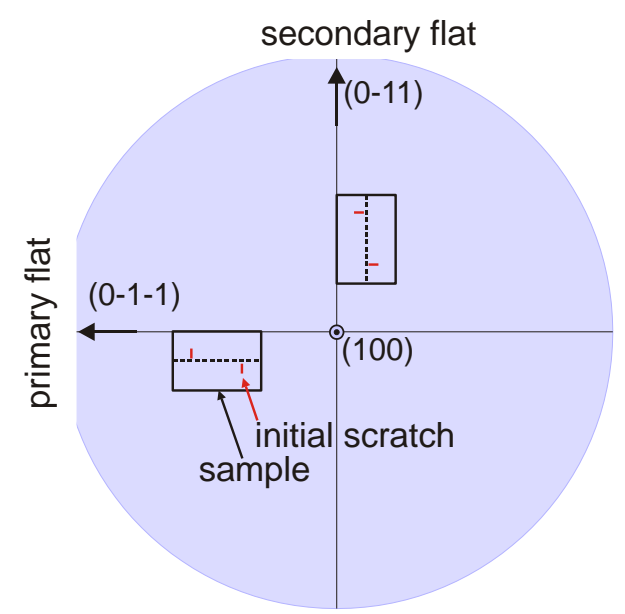

Figure 2.2: Sketch of the wafer direction. The solid black box shows the sample size before sample thinning; the dotted line indicates the cleavage afterwards. The short red line illustrates the position of initial scratch which is added before the samples are built in the sample holder.

illustrated in Figure 2.2. As the resulting surface investigated in the STM is different for the two directions, it is crucial to know the orientation of the pieces.

\subsubsection{Sample thinning}

The commercial GaAs wafer has a thickness of about $500 \mu \mathrm{m}$. For STM experiments, the samples have to be cleaved in UHV and a high quality of the cleavage surface with low step density and large atomically flat terraces is necessary. Especially samples with a heterostructure have to be thinned to obtain good cleavage results. The samples are chemically etched in a bromine-methanol solution until they have a thickness of about $100 \mu \mathrm{m}$. The bromine-methanol solution reacts with the GaAs and the surface is dissolved. During the etching process the samples are moved across filter paper, which is covered by the solution. By this procedure it is ensured that the etching products are immediately removed from the surface and the surface stays flat. A more detailed description of the thinning process can be found in [13]. After thinning, the $6 \times 9 \mathrm{~mm}$ sized samples are cleaved in the middle and one obtains 2 samples of $3 \times 9 \mathrm{~mm}$ sizes. Each sample has one clean cleavage side, which is not roughened by the thinning.

\subsubsection{Ohmic contacts}

The contacts have to be ohmic at low temperature. Furthermore a sample including a heterostructure is used and this structure should not be affected by the preparation of the ohmic contacts.

The GaAs sample is $\mathrm{n}$-doped, thus an AuGe (300mg) alloy and $\mathrm{Ni}(5 \mathrm{mg})$ was used to contact the sample. In a vacuum chamber working at $5 \cdot 10^{-6}$ mbar the AuGe alloy mixed with the Ni was evaporated by current heating with an evaporation distance of about $20 \mathrm{~cm}$. Both sides of the samples are contacted. The size of the contacts is a circle with a radius of about $1 \mathrm{~mm}$. 


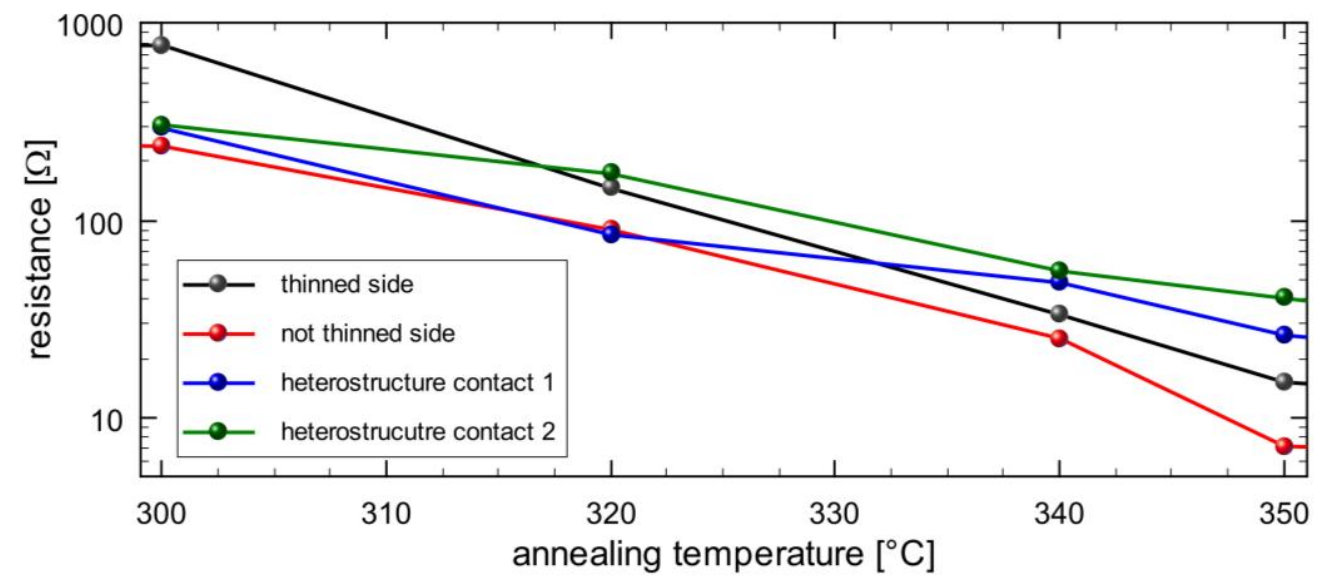

Figure 2.3: Resistance of the sample contacts as a function of annealing temperature.

Afterward the samples are annealed. During annealing, the contact metallization diffuses into the GaAs crystal. As the quality of the ohmic contact seemed to be crucial dependent on the annealing temperature, especially as higher temperature destroyed the possibility to apply a voltage across the heterostructure, a study of the resistance at room temperature as a function of annealing temperature was made (see Figure 2.3).

The following procedure has been used: The samples had been preannealed at $320^{\circ} \mathrm{C}$ and $340^{\circ} \mathrm{C}$ and if the measured resistance was lower than $100 \Omega$ the samples were annealed at $360^{\circ} \mathrm{C}$. If the resistance was larger than $100 \Omega$ the samples were first annealed at $350^{\circ} \mathrm{C}$ and then annealed at $360^{\circ} \mathrm{C}$. This procedure assured to receive ohmic contacts, which still work at low temperatures and does not destroy the heterostructure. The annealing time for each process was $150 \mathrm{~s}$.

\subsubsection{Initial Scratch and Sample cleavage}

Before the samples are inserted into the sample holder an initial scratch with a tungsten carbide tip was applied on the sample. The scratch produces dislocations in the sample and the crystal will usually cleave at this position when a force is exerted on the crystal. The scratch can be positioned at a special height on the sample by using a translation stage for the sample. The tip is fixed for lateral movement and can be put down on the sample with a defined force. The advantage of this procedure is that the initial scratch always has an exact position above the sample holder clamp jaws. The length is chosen to be $500 \mu \mathrm{m}$ and ends about $20-30 \mu \mathrm{m}$ in front of the sample edge. The height is chosen to be $2800 \mu \mathrm{m}$, which is $300 \mu \mathrm{m}$ above the sample holder clamp jaws. In Figure 2.4(a) a photograph of the sample holder including the sample is shown; Figure 2.4(b) shows the sample before mounting into the holder.

The sample is clamped between two gold plated copper clamp jaws, which are electrically isolated from each other. Each clamp jaw is connected to the contacts of the sample holder, which can be connected to the contacts of the STM. Thus, it is possible to apply a voltage across the sample. 

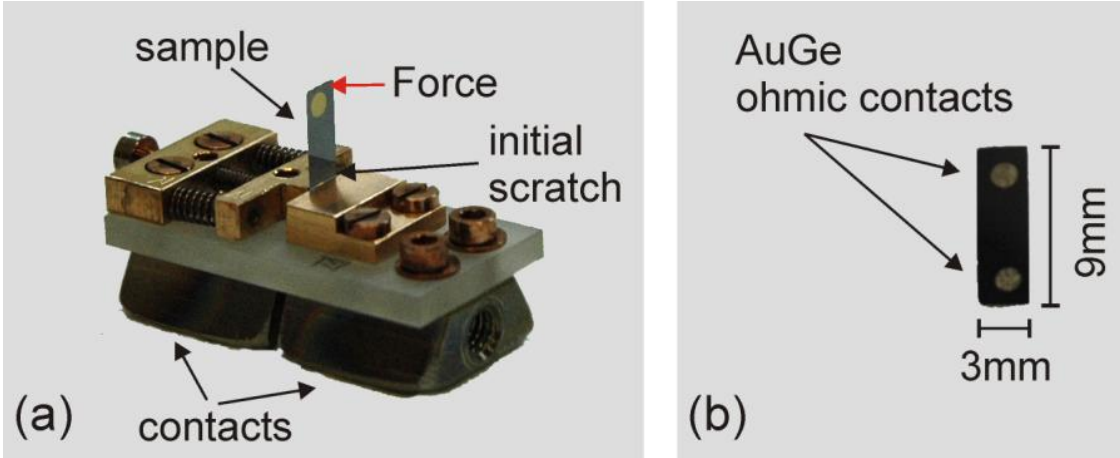

Figure 2.4: a) Sample holder with sample, the contacts of the sample holder are separated from each other. The position of the initial scratch and the direction of the force are shown. b) Sample showing the AuGe ohmic contacts.

The sample holder is transferred into the UHV-chamber. Before cleavage, the sample holder is rotated by $90^{\circ}$ so that the initial scratch is at the top. A force is exerted to the sample at the position shown in Figure 2.4. 


\title{
3. Charge switching of Si-donors in GaAs
}

\begin{abstract}
Donors in GaAs are ionized due to the potential induced by the STM tip. This charge switching process is investigated spatially resolved for single donors. The charge switching process of a donor is influenced by the presence of a second donor. This influence will be studied.
\end{abstract}

\subsection{Overview}

The charge state switching behavior of Si donors near the GaAs (110) surface is investigated with scanning tunneling spectroscopy (STS). Comparing the geometry of the STM tip and the GaAs sample with the functional element in gated semiconductor devices, the tip can be described as a movable gate which forms the space charge region inside the semiconductor. The microscopic process forming this space charge region is the subsequent ionization of individual dopants within the semiconductor. In the STM experiment the ionization process can be studied with Angstrom resolution and simultaneously with different gate voltages. The combined knowledge of the arrangement of dopant atoms, their charge state and the corresponding electrostatic potential as a function of gate voltage can lead to a deeper understanding of the active region of semiconductor devices.

Section 3.2 will give an overview of measurements on a single donor and a model of the ionization process is developed to explain the observations. A simulation of the space charge region, which is called in the case of STM the tip induced band bending (TIBB) is compared to the measurements to confirm the predicted ionization model. The interplay of the ionization process to the tunnel current allows one to extract the Coulomb potential of the positive charged donor in GaAs, which will be done in section 3.3. By the investigation of donors in different depth, it will be shown that the build up process of the space charge region does not start with the donor closest to the surface, but donors in deeper layers start to change their charge state first. This will be explained in section 3.4 by an enhanced binding energy for donors close to the surface. Section 3.5 will deal with the question of interacting donors. It will be shown that donors do not ionize independently from each other, but that they form ionization 
gaps and even bistability can be observed. The last section 3.6 will give a short overview of a more precise description, how the system should be treated in a quantum mechanical way.

\subsubsection{Description of shallow donors in bulk material}

The silicon donor in GaAs is a shallow donor. In the bulk material these donors are usually described by the hydrogen atom model. This description provides theoretical values for the ionization energy, as well as for the wave function [38]. The hydrogen atom model is only valid for bulk donors, as the potential of the surface changes their properties. But still the use of the hydrogen atom model for donors at the surface will be used in this thesis in a modified form to describe the ionization process. Theoretical calculations of shallow donors near an interface have been done for example by Hao et al. [39]. They investigated the ground state energy of donors in front of a metal/oxide layer; their result is a reduction of the binding energy with decreasing distance to the interface.

The donor has an additional charge compared to the atoms of the host material. In the hydrogen atom model this charge is simply described by a point charge with the well known Coulomb potential. The influence of the surrounding atoms is included by the screening due to the dielectric material using the dielectric constant $\varepsilon_{r}$ of the material and by the effective mass $m^{*}$ of electrons in the conduction band.

Using the dielectric constant of GaAs $\varepsilon_{i}=13.1$ and the effective mass $m^{*}=0.067 m_{0}$, the solution of the Schrödinger equation yields for the energy:

$$
E_{B}=\frac{m^{*} e^{4}}{32\left(\hbar \pi \epsilon_{0} \epsilon_{r}\right)^{2}}=E_{R y d} \frac{m^{*} / m_{0}}{\epsilon_{r}^{2}}=5.3 \mathrm{meV}
$$

Here $E_{R y d}$ is the Rydberg energy, and $m_{0}$ the free electron mass. The wave function expected for an electron bound to the donor is simply the 1s state of the free hydrogen atom with a modified Bohr radius $\left(a_{B}^{G a A s}\right)$ :

$$
a_{B}^{\mathrm{GaAs}}=\frac{4 \pi \hbar^{2} \epsilon_{0} \epsilon_{\mathrm{r}}}{e^{2} m^{*}}=a_{B} \frac{\epsilon_{\mathrm{r}}}{m^{*} / m_{0}}=10.3 \mathrm{~nm}
$$

Here $a_{B}$ is the Bohr radius of the donor atom in GaAs.

\subsubsection{Si doped GaAs investigated in STM}

The sample which is used for the investigation of this chapter is Si doped GaAs. Silicon is incorporated on the gallium site and is thus a donor in GaAs. The samples are provided from Freiberger Compound Materials $\mathrm{GmbH}$, The silicon doping is $6.5 \cdot 10^{18} \mathrm{~cm}^{-3}$. 
(a)

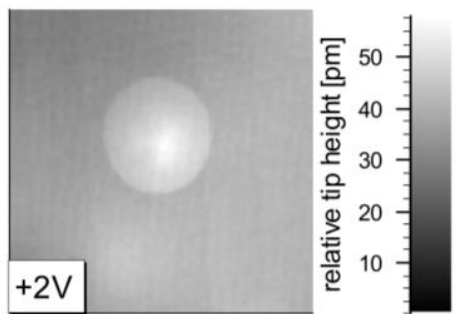

(b)
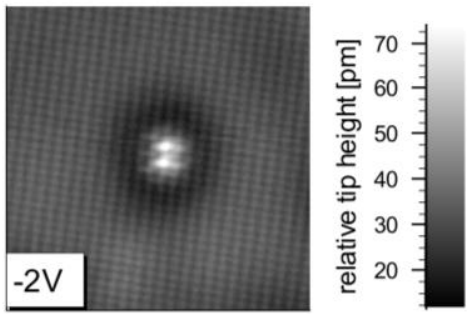

(c)

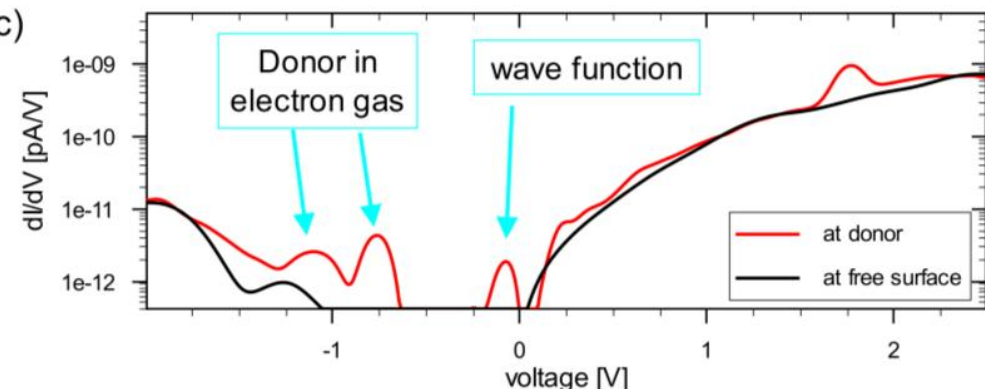

(d)

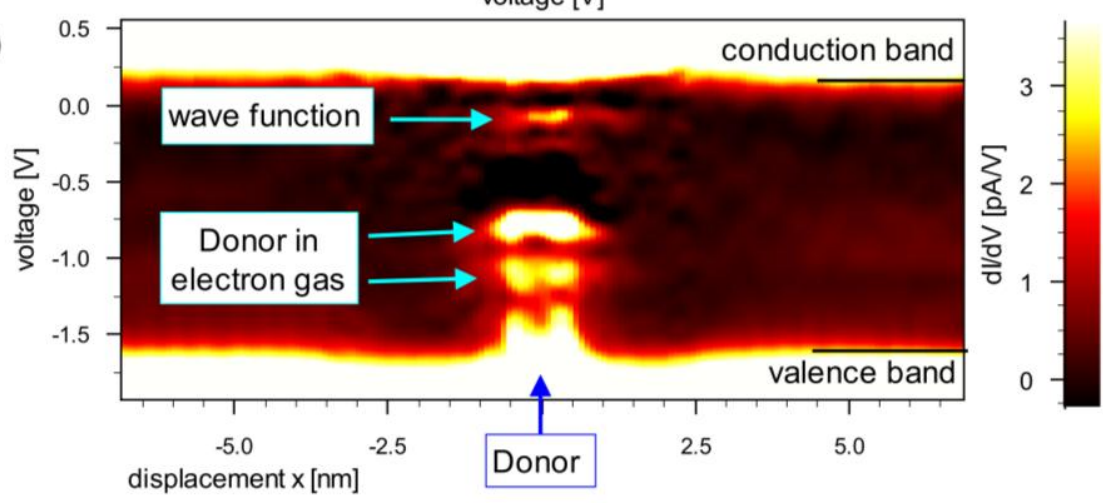

Figure 3.1: Identification of a donor in GaAs: $(a, b)$ constant current topography image at $+2 \mathrm{~V}$ and $-2 \mathrm{~V}(14 \times 14) \mathrm{nm}^{2}$ of the same donor. In (a) the charge switching of a donor is seen as a disk of enhanced topographic height around the donor center. In (b) Friedel oscillations appear around the donor, which is one way to identify donors. (c) $\mathrm{d} / \mathrm{d} V(V)$ spectroscopy taken directly at the donor center (red) and at the free surface (black). (d) Laterally resolved $\mathrm{d} / \mathrm{d} V(x, V)$-section (the corresponding topography image is taken at a voltage of $2.5 \mathrm{~V}$ and $0.5 \mathrm{nA}$ ); the donors' position is at $0 \mathrm{~nm}$. The spectroscopic feature can be used to identify a donor.

Figure 3.1 shows constant current topography image at a setpoint of (a) $+2 \mathrm{~V}$ and $100 \mathrm{pA}$ as well as (b) $-2 \mathrm{~V}$ and $100 \mathrm{pA}$ of the same donor. A disk of enhanced topographic height is visible around the donor center for positive voltage. The donor can be identified by its topographic contrast at negative voltage, where Friedel oscillations appear [40,19]. Spectroscopic features within the band gap taken directly above the donor give further indication for the identification of the donor. In Figure 3.1(c) a $\mathrm{d} I / \mathrm{d} V(V)$-spectroscopy taken directly above the donor center (red) is compared to a spectroscopy at the free surface ${ }^{3}$ (black). (Both shown spectra are averaged over several spectra; the corresponding topography image was taken at $2.5 \mathrm{~V}$ and $0.5 \mathrm{nA}$ ). The additional peak appearing at $-60 \mathrm{mV}$ can be assigned to the donor wave function. The peaks at $-0.7 \mathrm{~V}$ and $-1.2 \mathrm{~V}$ can be assigned to additional states of a positive donor

\footnotetext{
${ }^{3}$ The free surface should mean an area as far away as possible from any visible donor.
} 
core embedded in an electron gas, which occurs due to the TIBB. Figure 3.1(d) visualizes the data laterally resolved, it can be seen that the described peaks are only located around the position of the donor center (at $0 \mathrm{~nm}$ ).

\subsection{Principle of donor charge switching}

In this section a principle model for the ionization process of donors will be explained. The model is tested by comparing the measurements with a simulation of the TIBB. It has been published 2008 in [41].

Similar results of charge switching in an STM have been reported on different systems: Wildöer et al. [42] showed that charges can be induced on a small grain by the STM tip. Repp et al. [43] reported the manipulation of $\mathrm{Au}$ adatoms on a $\mathrm{NaCl}$ film, where they used a voltage pulse to switch between two geometric configurations; it was accompanied by a charge manipulation. For a system of an alkali doped $\mathrm{C}_{60}$ monolayer crystal grown on $\mathrm{Al}_{2} \mathrm{O}_{3}$ on a $\mathrm{NiAl}(110)$ substrate Pradhan et al. [44] showed charge switching. Very closely related is the ionization of Mn acceptors in InAs, which was published by Marczinowski et al. [45]. Ionization of atoms and molecules by STM is still under investigation for example Co atoms on graphene have been studied by Brar et al. [46] in 2011 and molecules on a $\mathrm{NaCl}$ bilayer by Swart et al. [47].

From now on the focus of the discussed measurements will be on positive voltage where the disk of enhanced topographic height appears. It will be shown that its origin is the ionization of the donor. The size of the disk of enhanced topographic height depends on the applied voltage, which is visualized in Figure 3.2. In Figure 3.2(a) and (b) the size dependence can be seen by two constant current topography images of the same donor at two different voltages $(+2 \mathrm{~V}$ and $+1.6 \mathrm{~V})$. Topography sections of five different voltages at the same donor are compared in Figure 3.2(c). The topographic sections show that the edge of the disk appears as an instantaneous step, indicated by the arrows in Figure 3.2(c).

The size of the disk is also dependent on the current setpoint, which changes the tipsample distance. This is shown for two topography cross sections in Figure 3.3 which are taken at the same voltage of $2.5 \mathrm{~V}$ and two current setpoints $0.1 \mathrm{nA}$ (black) and $0.5 \mathrm{nA}$ (red). (For both lines the free surface is set to be 0pm height). With decreasing distance between tip and sample the size of the disk increases, which is indicated by the arrows. 

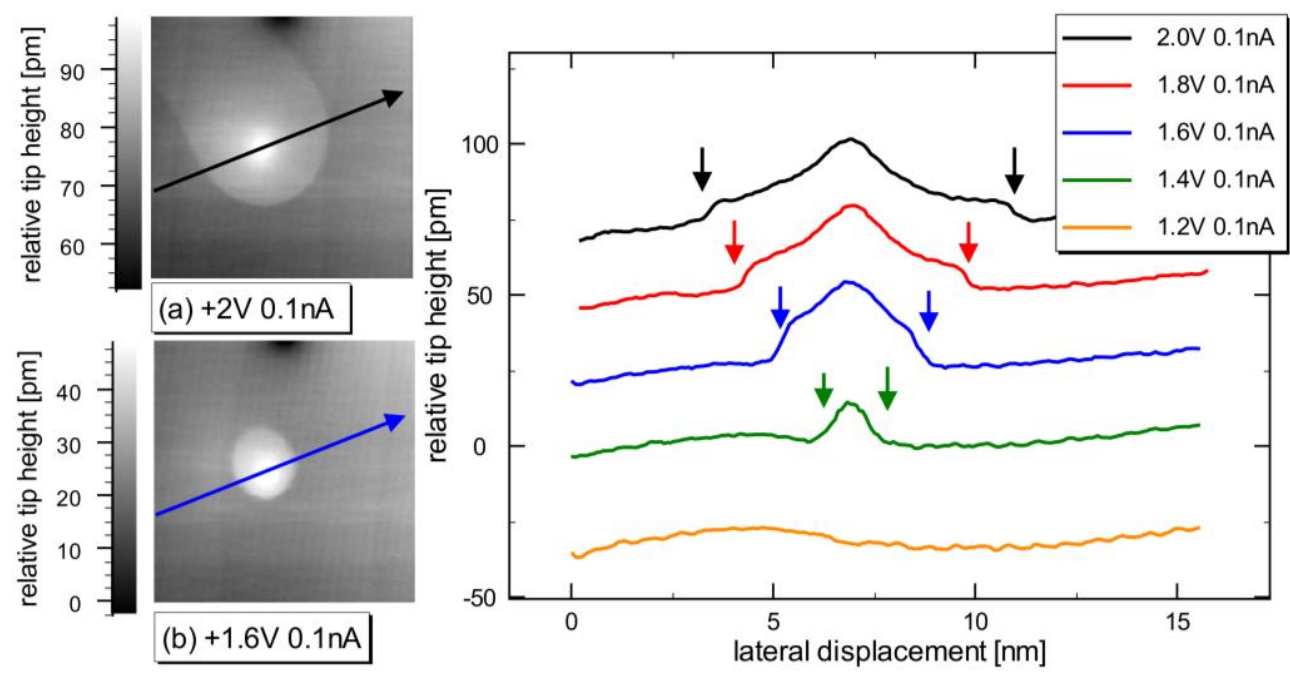

Figure 3.2: Voltage dependence of the disc size: Two constant current topography images of the same donor at a setpoint of $(\mathrm{a})+2 \mathrm{~V}$ and $(\mathrm{b})+1.6 \mathrm{~V}$ and $100 \mathrm{pA}$ are shown $\left(15 \times 15 \mathrm{~nm}^{2}\right)$. The size of the disk of enhanced topographic height is dependent on the applied voltage. In (c) topography cross sections through that donor are shown for different voltages. At the edge of the disk a jump in the topographic height is visible, indicated by the arrows. For a voltage of $1.2 \mathrm{~V}$ no jump occurs.

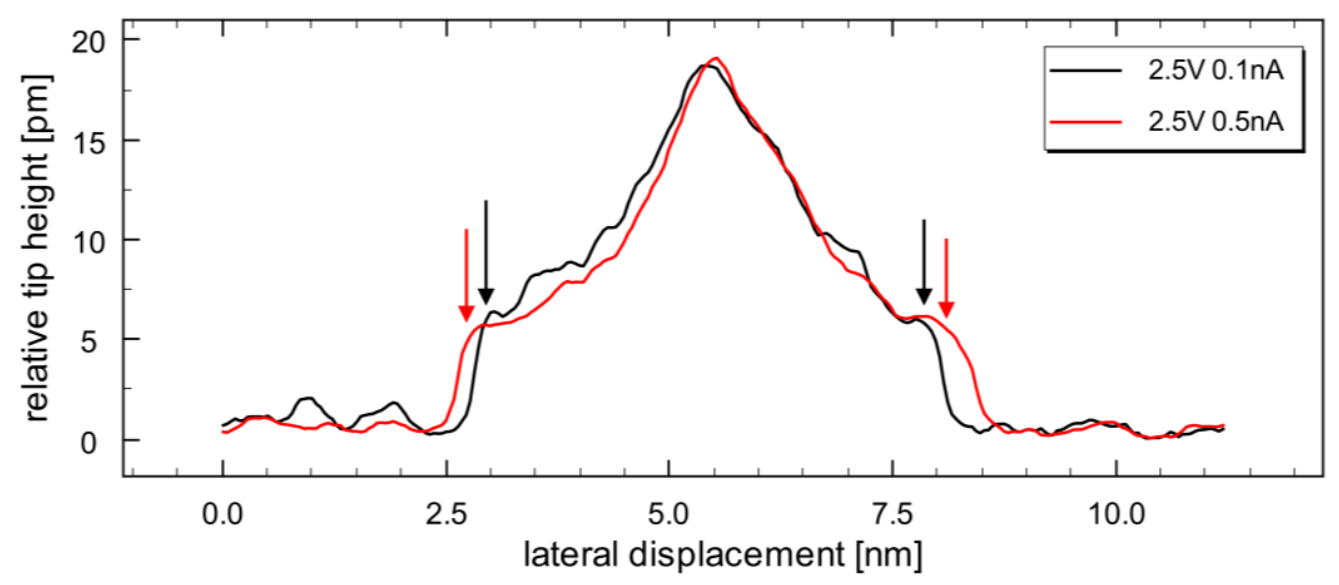

Figure 3.3: Current dependence of the disk size: two topography cross sections through a donor for the same voltage $(2.5 \mathrm{~V})$ but different current setpoints $0.1 \mathrm{nA}$ (black) and $0.5 \mathrm{nA}$ (red). With enlargement of the current, the tip gets closer to the surface and the disk of the donor enlarges.

Figure 3.4 illustrates a model of the ionization mechanism. When the tip is laterally far away from the donor, as shown in Figure 3.4(a), the bands on top of the donors are flat and are not influenced by the tip. The energy band diagram perpendicular to the surface is shown in Figure 3.4(b) for that case. Since the measurements are done at $5 \mathrm{~K}$, the thermal energy is much smaller than the ionization energy and the donor is neutral. If the tip is close to the donor, as shown in Figure 3.4(c), the bands are lifted at positive sample bias, and the donor level is pulled up as well. At a critical voltage 


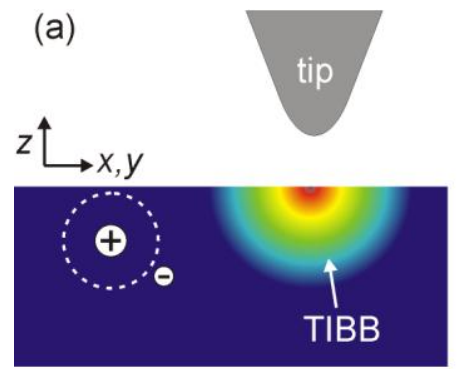

\section{(c)}
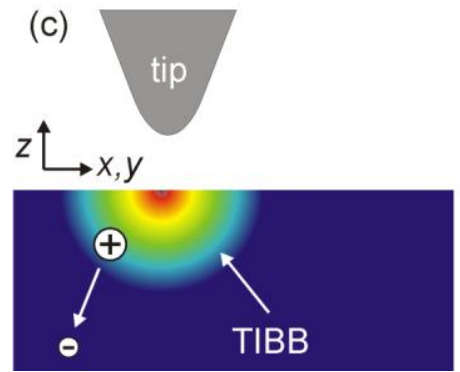

(b)
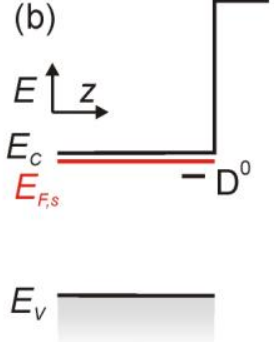

(d)

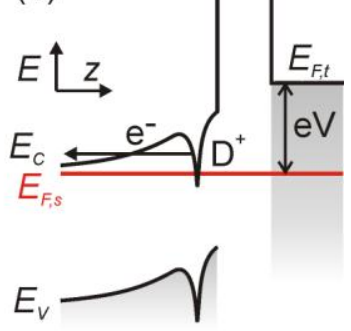

Figure 3.4: Schematic representation of the ionization mechanism: When the tip is laterally far away from the donor (a), the bands on top of the donors are flat (b) and the donor will be neutral. As the tip approaches laterally the donor with a positive sample bias (c), the bands are lifted due to the TIBB (d). At a certain voltage the donor level aligns with the conduction band in the bulk and the electron can escape.

$V_{c}$ the donor level is pulled above the onset of the conduction band in the bulk. The electron can now tunnel into the conduction band, which is illustrated in Figure 3.4(d). The Coulomb field of the ionized donor causes the bands at the surface to drop, therefore the amount of states available for tunneling enhances. This results in an instantaneous enhancement of the tunnel current, leading to a retraction of the tip, which is visible as the disk in Figure 3.2.

In this model the amount of TIBB on top of the donor is the crucial parameter to ionize the donor. The critical amount of TIBB is labeled $\operatorname{TIBB}_{\mathrm{c}}$. It can be manipulated in different manners:

- Changing the lateral distance between the tip and the donor, simply by moving the tip laterally. This changes the TIBB on top of the donor.

- Reducing the tip-sample distance, this also enhances the TIBB.

- Increasing the applied voltage, this enhances the TIBB. At sufficiently low voltage the donor is neutral, and above a critical voltage $V_{c}$ it is ionized.

All three methods have been experimentally explored in detail and quantitatively.

The ionization by laterally approaching the donor can be seen in constant current topography images. The edge of the disk in Figure 3.2(c) represents the ionization of the donor. The disk diameter depends on the depth of the donor below the surface (compare section 3.4). The ionization process by reducing the tip-sample distance was experimentally shown in Figure 3.3. 


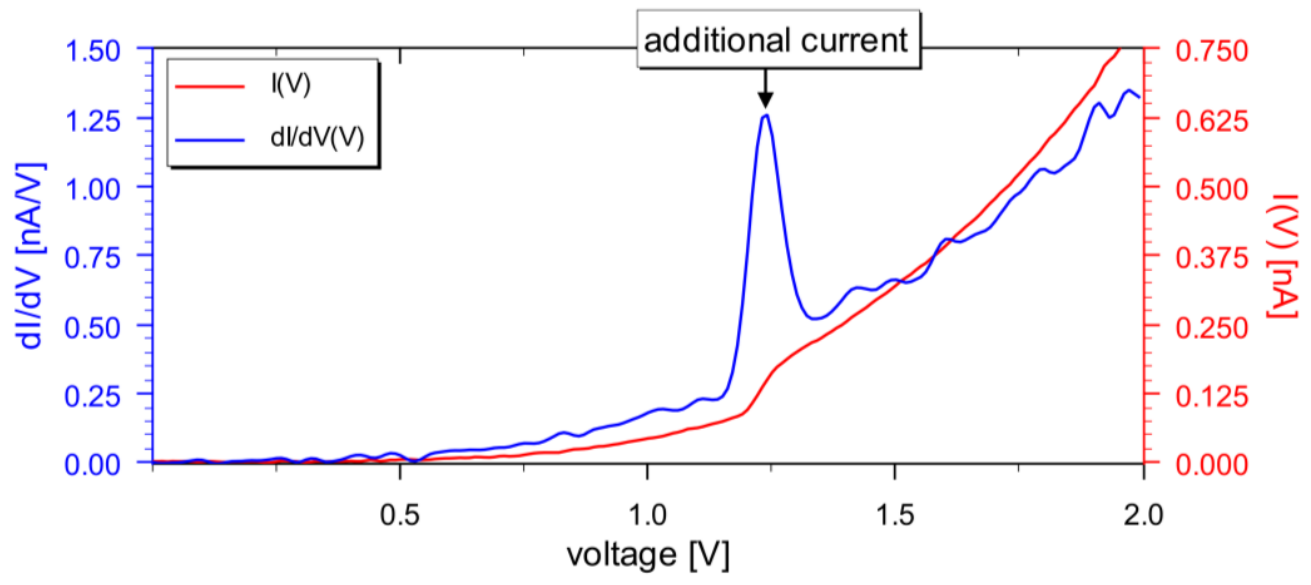

Figure 3.5: Ionization process visualized in $I(V)$ spectroscopy. It is recorded directly at the center of a donor. At 1.3V the jump in the red $I(V)$-curve and the peak in the corresponding blue $\mathrm{d} / / \mathrm{d} V(V)$-curve indicates the charge transfer of the system.
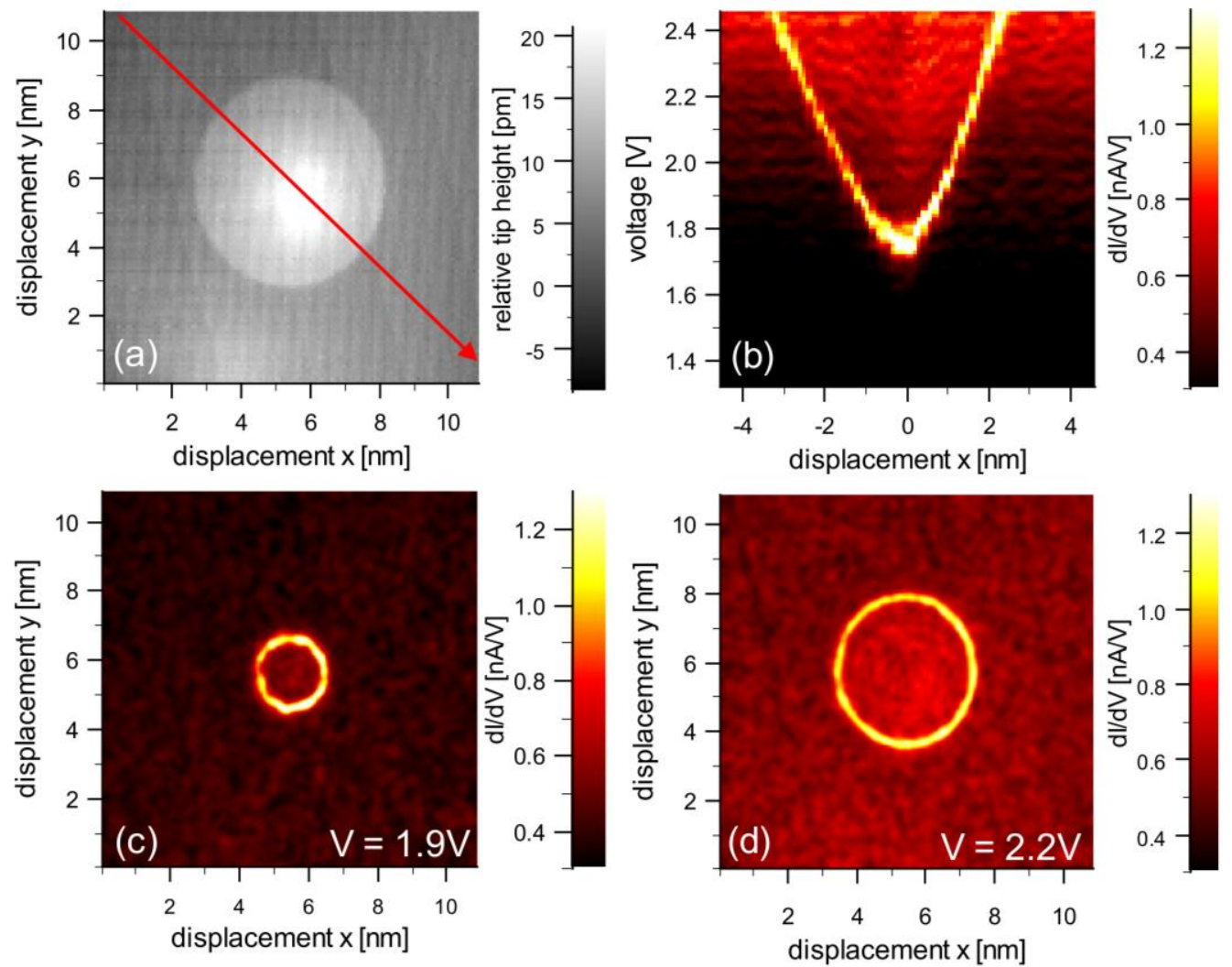

Figure 3.6: In (a) a constant current topography image of a donor at a voltage of $2.5 \mathrm{~V}$ and current 500pA is shown. The red line indicates the lateral position of the $\mathrm{d} / / \mathrm{d} V(x, V$-section in (b). Images (c) and (d) are laterally resolved $\mathrm{d} / / \mathrm{d} V(x, y)$-maps at different voltages. Higher differential conductivity is seen as a ring around the donor center, the ring diameter increase with voltage. Image (b) shows a $\mathrm{d} / / \mathrm{d} V(x, V)$-section along the red line in (a). The curve in (b) is the ring in (c) and (d). 

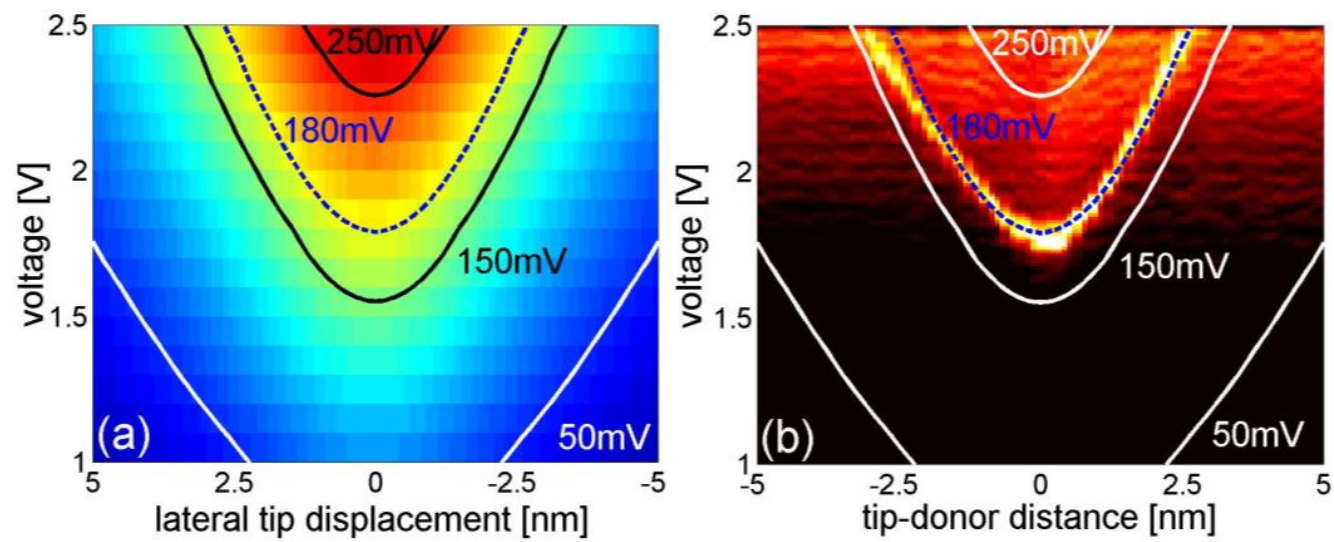

Figure 3.7: (a) Simulated $\operatorname{TIBB}(x, V)$ (color-coded $0-300 \mathrm{mV}$ ) as a function of lateral displacement of the tip and voltage $\mathrm{V}=\phi_{\text {sample }}-\phi_{\text {tip. }}$. The solid lines are contour lines of constant TIBB. (b) Measured $\mathrm{d} / \mathrm{d} V(x, V)$-section (color-coded 0.3-1.3 nA/V) as a function of lateral displacement of the tip to the donor center and voltage. The contour lines of (a) are included in the image (b).

The ionization process lateral and voltage dependent can be studied nicely in $I(x, y, V)$ spectroscopy. Here the ionization is seen as a jump in the $I(V)$-curve, or as a peak in the $\mathrm{d} I / \mathrm{d} V(V)$-curve (Figure 3.5). The explanation of the jump is the same like for the topography image: By increasing the voltage the TIBB on top of the donor increases as well. If the critical amount of $\mathrm{TIBB}_{\mathrm{c}}$ is reached the donor is ionized. The Coulomb potential of the ionized donor pulls the bands downwards and additional states are available for tunneling, which enhances the tunnel current instantanly.

In lateral resolved spectroscopy measurements (STS) the lateral and voltage dependent ionization can be studied. Figure 3.6(a) shows a topography image of an STS measurement. The peak, which is caused by the ionization, is seen as a bright ring around the donor center in the differential conductivity $\mathrm{d} I / \mathrm{d} V(x, y)$-maps in Figure 3.6(c) and Figure 3.6(d) (1.9V and 2.2V). This ring is similar to the disk of enhanced topographic height in the topography image. The images confirm that the diameter increases with voltage. Figure 3.6(b) shows a lateral resolved $\mathrm{d} I / \mathrm{d} V(x, V)$-section along the red line in Figure 3.6(a). The hyperbola of higher differential conductivity corresponds to the diameter of the ring as a function of voltage. According to the proposed mechanism the donor ionizes at a critical $\mathrm{TIBB}_{\mathrm{c}}$, therefore we expect the ring to follow a line with constant TIBB.

A simulation of the $\operatorname{TIBB}(x, V)$ as a function of voltage and distance of the donor to the tip is shown in Figure 3.7(a). The simulation was done by a tool to solve the Poisson equation of the three dimensional tip-sample geometry. The solver is provided by Feenstra [20]. Contour lines of constant amount of TIBB are highlighted in the image. These contour lines are added to the measured $\mathrm{d} I / \mathrm{d} V(x, V)$-section, which can be seen in Figure 3.7(b). To extract the flat band condition, which is an essential parameter, the apparent barrier height was measured simultaneously with the STS data. For the data set shown in Figure 3.7(b) it is assumed that the flat band condition is at $0.1 \mathrm{~V}$, which is due to the fact that the work-function of bulk tungsten strongly differs if the surface is not perfectly flat $[48,49]$. In Appendix A.1 the voltage of the flat band 


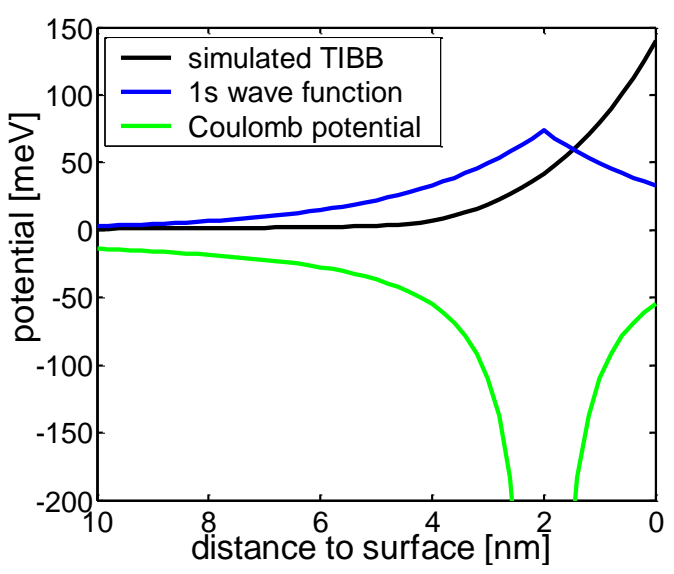

Figure 3.8: Simulated TIBB (black line) for a voltage of $1.0 \mathrm{~V}$. Coulomb potential of a positive charge in GaAs (green line). 1s wave function of the hydrogen model (blue line). The spatial extension of the wave function is in the order of the spatial extension of the simulated TIBB.

condition is discussed in more detail. For the measurement shown in Figure 3.7(b) the ring follows a calculated TIBB of $180 \pm 50 \mathrm{meV}$. The TIBB calculations strongly depend on the tip-sample distance, the tip shape, the flat band condition, and the (local) doping concentration. Some of these input parameters are unfortunately not known accurately in STM experiments. Within the range of reasonable values no unique fit can be found for the simulation, due to correlations in the parameter space. This range of possible TIBB values results in the uncertainty of $\pm 50 \mathrm{meV}$ in the simulation.

\subsubsection{From TIBB to donor binding energy}

The ionization of donors in a space charge region is usually described by the binding energy of the electron to the donor. An external potential shifts the bound state above the Fermi energy. The amount of external potential needed to ionize the donor is the same like the donor binding energy, which is $6 \mathrm{meV}$ for bulk GaAs donors. Up to now the above describe model considers only the TIBB at the donor center for the ionization process. Assuming a simple shift of the bound state one could conclude that the binding energy of a donor close the surface is enhanced to $180 \mathrm{meV}$, which is dramatically large. A more precise consideration shows that that the spatial extension of the wave function and the decaying of the TIBB into the sample are on the same order of magnitude. This is visualized in Figure 3.8. The amount of TIBB is changing significantly in the region of the wave function. Therefore a simple shift caused by the TIBB value at the donor center is not a good approximation. Instead the shift of the binding energy could be better estimated by the mean value of the TIBB in the region of the wave function, which is explained in the following paragraph and has been published in [54].

As a rough estimation, one can treat the problem by first order time independent perturbation theory, (this theory can be found in any standard quantum mechanics book for example [50]). For the unperturbated system the model of the hydrogen atom is used, the perturbation is the TIBB. The energy shift $\Delta E_{B}$ of the lowest bound state is an integral over the whole space $\mathbf{r}$ : 


$$
\Delta E_{B}=\iiint \Psi_{1 s}(\mathbf{r}) \operatorname{TIBB}(\mathbf{r}) \Psi_{1 s}^{*}(\mathbf{r}) d r^{3}
$$

The $\operatorname{TIBB}(\mathbf{r})$ has been simulated and is used to calculate the integral. A little more discussion is needed for the choosing of the wave function. If the binding energy in the vicinity of the surface is enhanced, its Bohr radius will decrease compared to the bulk value of $\sim 10 \mathrm{~nm}$. Therefore a Bohr radius decreased by a factor of 2 was assumed for the $1 \mathrm{~s}$ wave function. Another point is that the effect of the surface is neglected for this analysis. In contrast to the bulk donor, the Coulomb potential for a donor at the surface is modified even without the tips potential. This might squeeze the whole wave function and shift its extension away from the surface into the bulk.

Anyhow this rough estimation is used to explain the difference of the TIBB and the binding energy: A donor with a distance of $2 \mathrm{~nm}$ to the surface is assumed to ionize at a voltage of $1 \mathrm{~V}$ (Figure 3.8). The TIBB directly at the surface is $150 \mathrm{meV}$; the TIBB at the position of the donor is $42 \mathrm{meV}$. Calculating $\Delta E_{B}$ for that special case according to equation (3.3) one obtains $17 \mathrm{meV}$. In this case the binding energy would be by a factor of 2.5 smaller than one would expect it according to the TIBB at the donors' position and even by a factor of 10 smaller according to the TIBB at the surface.

\subsection{Extracting the Coulomb potential}

In this section it is described how to use the jumps in the tunnel current in the STS data to obtain detailed spatial information of the donor's electrostatic potential. The Coulomb potential of a single atomic charge $\mathrm{e}^{+}$is mapped with Angstrom resolution.

In Figure 3.9(a) spectra on the free surface (solid line) are compared with spectra taken at the donor center (dotted line). All spectra are normalized to a flat plane to remove any crosstalk from the topographic contrast of the donor (compare subsection 2.2.4). In the spectrum taken directly on top of the donor a sharp current jump is visible at about $1.3 \mathrm{~V}$ which is caused by the ionization of the donor. At voltages below $1.3 \mathrm{~V}$ the curves overlap. For higher voltages the ionized donor gives rise to an enhanced current. This is schematically shown in the inset in Figure 3.9(a). Due to its positive charge the ionized donor pulls down the bands at a voltage $V_{2}$. This enhances the number of states available for tunneling, and thus enhances the tunnel current, compared to the free surface. On the free surface a higher voltage $V_{1}$ is needed to obtain the same amount of states available for tunneling, which results in the same amount of current $I$. The data curve after the jump is manually shifted by a voltage $\Delta V$, until the current is the same (dashed line). The two curves overlap perfectly for higher voltages. This voltage shift directly gives the Coulomb potential originating from the ionized donor. For different distances to the donor center this voltage shift is extracted. The result is shown in Figure 3.9(b), the measured voltage shift (black dots) are plotted as a function of lateral position to the donor center. In the model the measured voltage shift is the Coulomb potential of the donor directly at the surface. According to this assumption the data are fitted by a cut through the theoretical expected Coulomb potential in a distance $z_{0}$ from the donor center: 


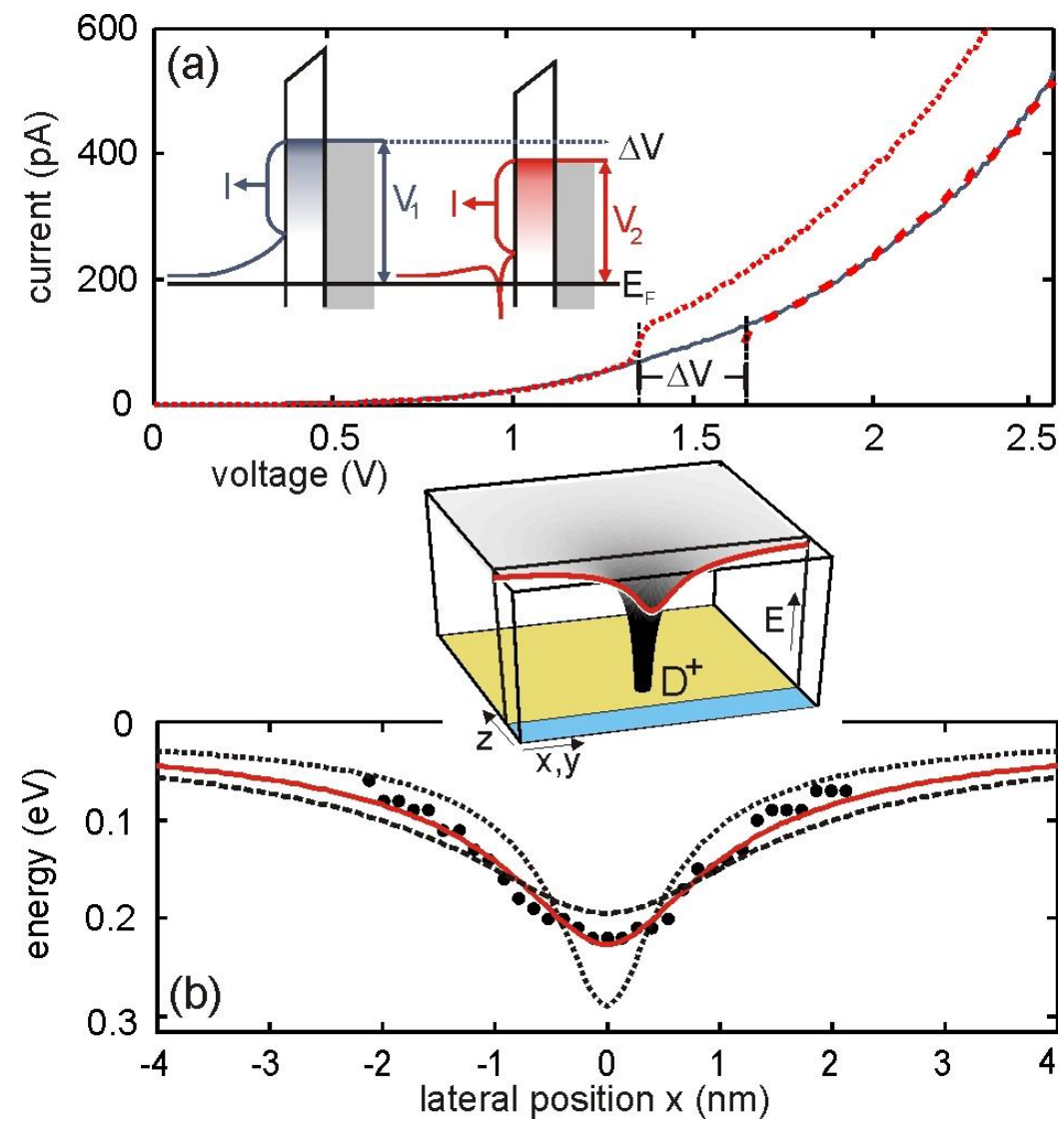

Figure 3.9: A spectrum taken directly above the donor (dotted red line) and a spectrum taken on the free surface (solid blue line) are shown in (a). At voltages lower than 1.3V the curves overlap. The curves overlap for higher voltages as well, by shifting the spectrum taken above the donor by a certain voltage (dashed red line). The situation is schematically shown in the upper inset. The voltage shift directly gives the Coulomb potential originating from the ionized donor. The voltage shift is extracted as a function of lateral distances to the donor center. The results are the dots in (b). The data is fitted with the Coulomb potential directly at the surface; see solid line in the lower inset. The three curves correspond to different donor depths: $4 \AA$ and $\varepsilon_{\mathrm{r}}=12.4$ (dotted line), $8 \AA$ and $\varepsilon_{\mathrm{r}}=8$ (red solid line), $12 \AA$ and $\varepsilon_{\mathrm{r}}=6.2$ (dashed line).

$$
\Phi_{\text {Coulomb }}\left(x, z_{0}, \varepsilon_{r}\right)=\frac{1}{4 \pi \varepsilon_{0} \varepsilon_{r}} \frac{1}{\sqrt{\left(x^{2}+z_{0}^{2}\right)}}
$$

Without loss of the generality the coordinate $y$ was set zero, then $x$ is the lateral position of the tip. The potential is illustrated with the red line in the inset of Figure 3.9(b).

The dielectric constant $\varepsilon_{\mathrm{r}}$ is used as a fitting parameter, which is explained in the following paragraph. The potential of a charge which is near an interface of a dielectric and the vacuum can be solved analytically with an electrostatic approach using image charges [51]. According to this solution the potential in the vacuum area - which includes the plane directly at the interface - should behave like the Coulomb potential of a charge at the same position like the original charge, with a modified dielectric constant: 


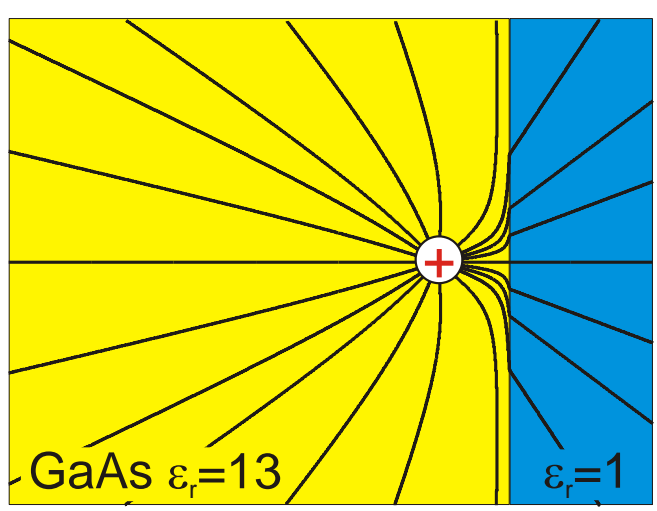

Figure 3.10 Field lines of a positive point charge in a dielectric close to the vacuum. The dielectric constant of GaAs does not change close to the surface. According to the electrostatic solution the potential in the vacuum behaves like a Coulomb potential with a modified dielectric constant.

$$
\varepsilon_{r}^{*}=\frac{\left(\varepsilon_{r, v a c}+\varepsilon_{r, G a A s}\right)}{2 \varepsilon_{r, v a c}}
$$

In GaAs the bulk value of $\varepsilon_{\mathrm{r}}=13.1$ [25], thus we would expect to find a value of about $\varepsilon_{\mathrm{r}}{ }^{*}=7$. Note that using the effective dielectric constant does not mean that the relative permittivity of GaAs itself changes close to the surface. For this description the reduction of the dielectric constant is just due to the electrostatic solution of the half space geometry. This is illustrated by the field lines of a charge in a dielectric close to the vacuum shown in Figure 3.10.

The second unknown parameter, which is used for the fitting is the depth of the donor $z_{0}$. The best fit for both parameter (donor depth and dielectric constant) of the data was obtained for a donor depth of $8 \AA$ below the surface and a dielectric constant of $\varepsilon_{\mathrm{r}}=8$. The best fit is shown as the red solid line in Figure 3.9(b). For comparison two further fits are presented. Here the distance of the donor was set to a fixed value of $4 \AA$ and $12 \AA$, and the only free parameter was the dielectric constant. This is shown for the donor depth of $4 \AA$ with the best fit of $\varepsilon_{\mathrm{r}}=12.4$ (dotted line) and for the donor depth of $12 \AA$ with the best fit of $\varepsilon_{\mathrm{r}}=6.2$ (dashed line). The measurement was performed on three different donors. In all measurements the value of $\varepsilon_{\mathrm{r}}$ for the best fit was $8 \pm 1$. This is close to the expected value following the classical half space approach.

Additionally, a topography image taken on this donor at negative voltages was used to determine that the donor is in an even layer ${ }^{4}$. This is done by comparing the maximum of the donor contrast with the atomic corrugation [52, 53]. If both maxima are at the same position the layer is even, otherwise the layer is odd (compare also Figure 3.16). For determining the distance of the donor to the surface, one has to know the absolute position of the surface. Usually the centers of the atoms in the first monolayer are used as the position of the surface. This would mean that a donor in an even layer has a distance of $(n \cdot 4 \AA)-2 \AA(=2 \AA, 6 \AA, 10 \AA, 14 \AA$, etc.). The best fit of the Coulomb potential for the donor was obtained for a layer depth of $8 \AA$. This could be a hint that the surface position is not that easy to define as it is usually done. This is illustrated in Figure 3.11. Here the barrier is shown as it is used for the Coulomb potential

\footnotetext{
${ }^{4}$ labelling the surface layer as $1^{\text {st }}$ layer
} 


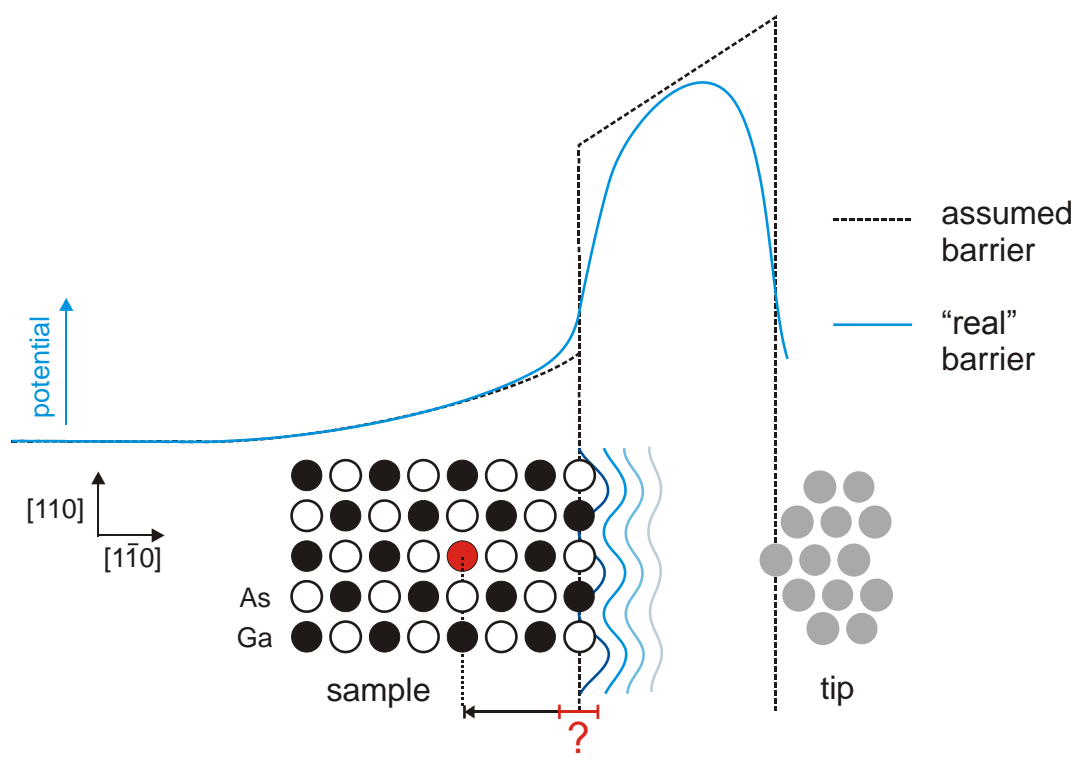

Figure 3.11: The upper part sketches the potential landscape. The dashed line shows the assumed barrier with sharp edges; the blue line sketches the more realistic picture of a smooth barrier. The lower part illustrates the atomic position in GaAs, with a donor in the $4^{\text {th }}$ monolayer (red). The blue lines in front of the atoms visualize the electrons decaying into the vacuum.

extraction (dashed line) and a more realistic view of a smooth barrier (blue line). In the region of the surface the abrupt barrier is not valid. For the analysis this might shift the position of the extracted Coulomb potential into the surface. In the lower part of the image, the atomic positions are sketched. It is not quite clear where the surface begins, as the electrons usually decay into the vacuum. Because of this problem, the analysis of the even and odd layer is useful for comparing donor with each other as it was done for example in [36]. It is not useful to extract an absolute distance from the surface.

\subsection{Enhancement of the binding energy}

The critical $\mathrm{TIBB}_{\mathrm{c}}$ is evaluated for donors in different depth below the surface. The analysis of this data leads to the conclusion that the binding energy for donors close to the surface is enhanced, which has been published in [54].

Figure 3.12 (a) shows a constant current topography image $\left(65 \times 65 \mathrm{~nm}^{2}\right)$ at a bias voltage of $1.8 \mathrm{~V}$ and a setpoint of $0.3 \mathrm{nA}$. A spectroscopy measurement was taken in this area. Several donors can be identified in this image seven donors are labeled, the yellow lines show the position of the $\mathrm{d} I / \mathrm{d} V(x, V)$-sections, which are shown in the same image. By the topographic height of the donors at negative voltage, the donors are divided into different layer depth, which is indicated by the number in the figure. 

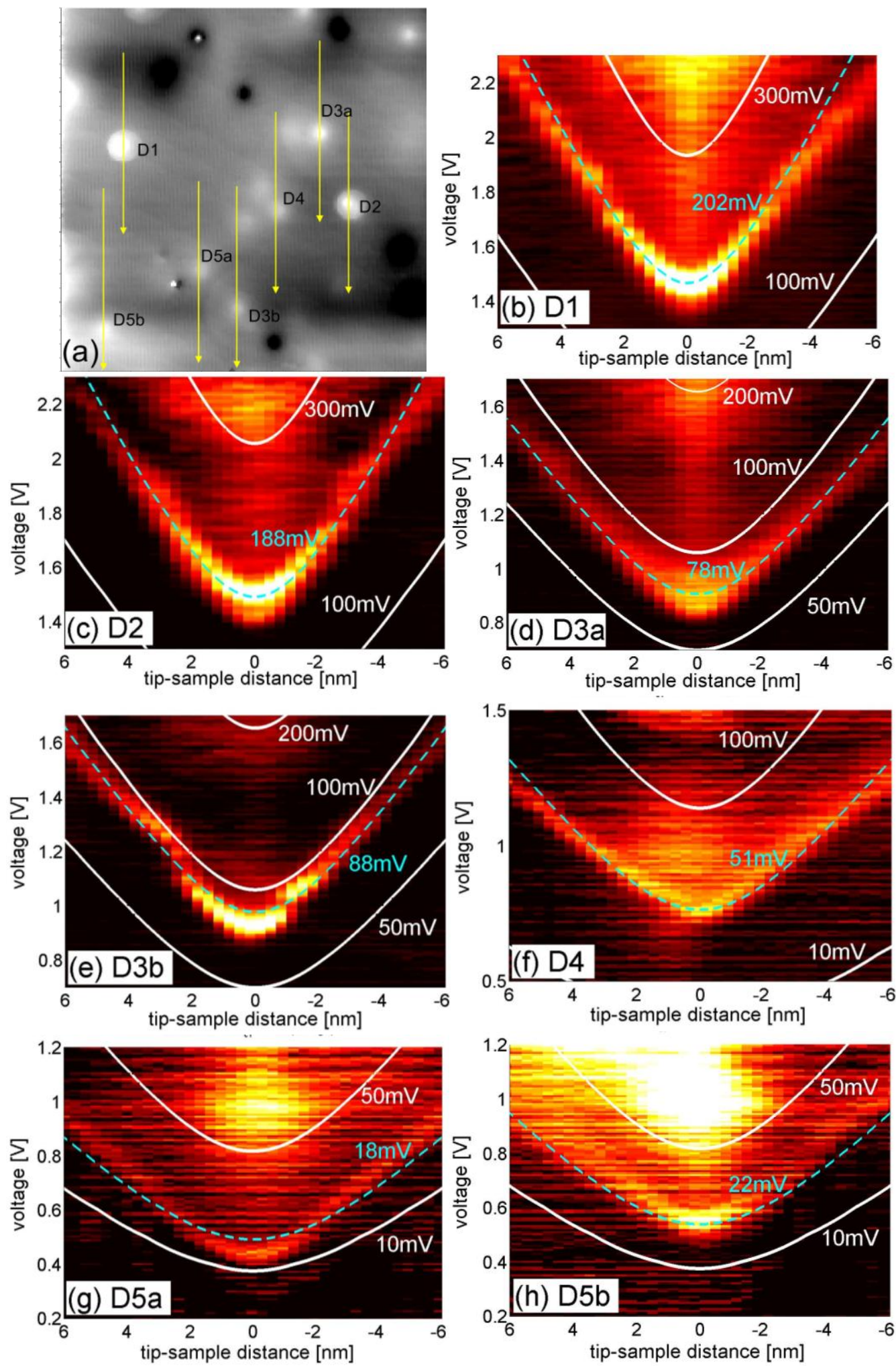

Figure 3.12: The grayscale image (a) shows a topography image $\left(65 \times 65 \mathrm{~nm}^{2}\right)$ of several donors; the yellow line shows the position of the spectrum sections. The color scale images (b-h) show $d / / d V(x, V)$-sections, the number of each donor is labeled in the topography and in the sections. The color scale is $0-0.8 \mathrm{nA} / \mathrm{V}$ for $\mathrm{D} 1,0-0.6 \mathrm{nA} / \mathrm{V}$ for D2, $0-0.4 n A / V$ for D3, $0-3 n A / V$ for D3b, $0-0.15 n A / V$ for $D 4$ and $0-0.1 n A / V$ for D5a and D5b. 

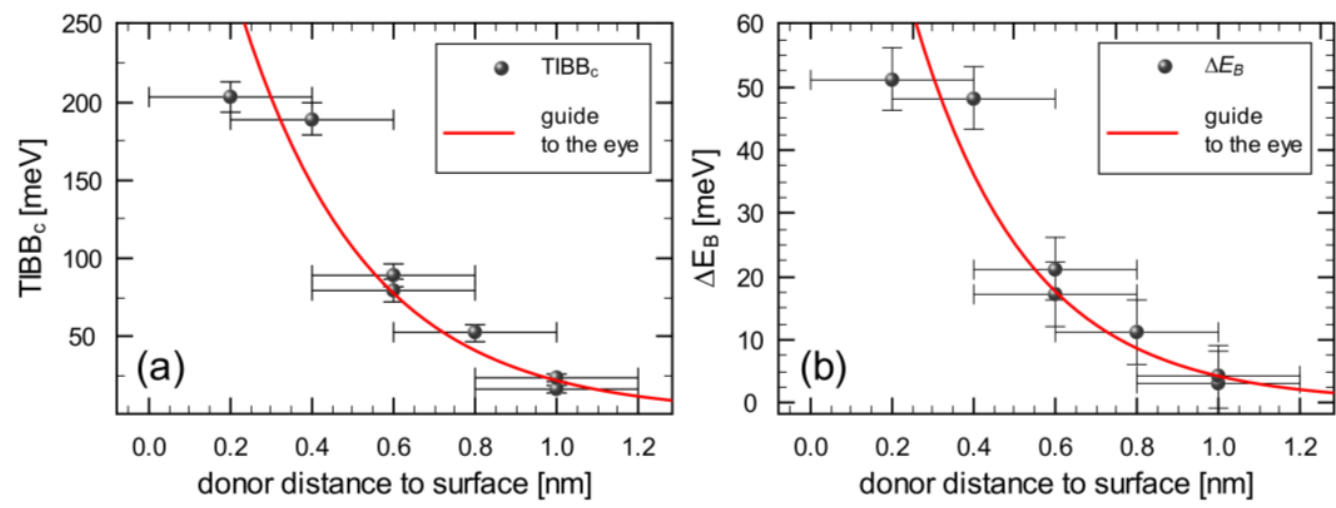

Figure 3.13: (a) Critical $\mathrm{TIBB}_{\mathrm{c}}$ as a function of donor distance to the surface. The red line is a guide to the eye. Donors close to the surface need a higher critical TIBB $_{c}$ to get ionized. The error bars of the $\mathrm{TIBB}_{\mathrm{c}}$ is the deviation if the donor is in a different layer. (b) Shift of the binding energy according to perturbation theory.

The differential conductivity sections Figure 3.12(b-h) are fitted to the TIBB in the same way like in section 3.2. The simulation parameters are the same for all seven donors. (Tip radius $3 \mathrm{~nm}$, tip angle $136^{\circ}$, tip-sample distance $0.7 \mathrm{~nm}$, flat band condition $100 \mathrm{meV}$, doping $5 \cdot 10^{18} \mathrm{~cm}^{-3}$ ). Even if the TIBB simulation has an error, the values can be compared among each other as the same parameter set was used. In Figure 3.13(a) the extracted values are plotted as a function of distance to the surface. The error bars in $\mathrm{x}$-direction are due to the possible uncertainty of the depth determination; the error bars in y-direction give the deviation of the critical $\mathrm{TIBB}_{\mathrm{c}}$ if the donor is in a different depth for the same simulation parameters. The red line is meant as a guide to the eye.

The trend of the data points is that the donors close to the surface need a much higher critical $\mathrm{TIBB}_{\mathrm{c}}$ to get ionized than donors deeper in the surface. This can be explained by an enhanced binding energy of the donors close to the surface. The critical TIBB of a donor does not directly give the binding energy. This is due to the fact that the amount of TIBB decreases into the semiconductor. In subsection 3.2.1 it was described how the binding energy can be evaluated from the critical amount of TIBB. The expected change of the binding energy according to equation (3.3) is plotted in Figure 3.13(b). This has also been shown in [54].

The change of the binding energy for donors close to the surface can be explained by two competing effects, which both change the potential of a donor. The first effect is due to the different dielectric constant in the GaAs half-space and the vacuum, which was already discussed in section 3.3. The electrostatic solution perpendicular to the surface is shown in Figure 3.14(a) (green curve). The donor is located in a distance of $1 \mathrm{~nm}$ to the surface. The potential is compared with the potential of a donor in GaAs (red curve) and the potential of a donor in GaAs having a double charge (blue curve). The solution of the half space geometry is close to the potential of the donor with a double charge. A double charge enhances the binding energy by a factor of four.

The second effect is the change of the potential due to the work function of GaAs. The potential of the donor is cut at the surface. This is illustrated in Figure 3.14(b) (green curve) and compared with the potential without the work function (dotted line). Comparing this scenario with the problem of a particle in a box, a reduction of the 

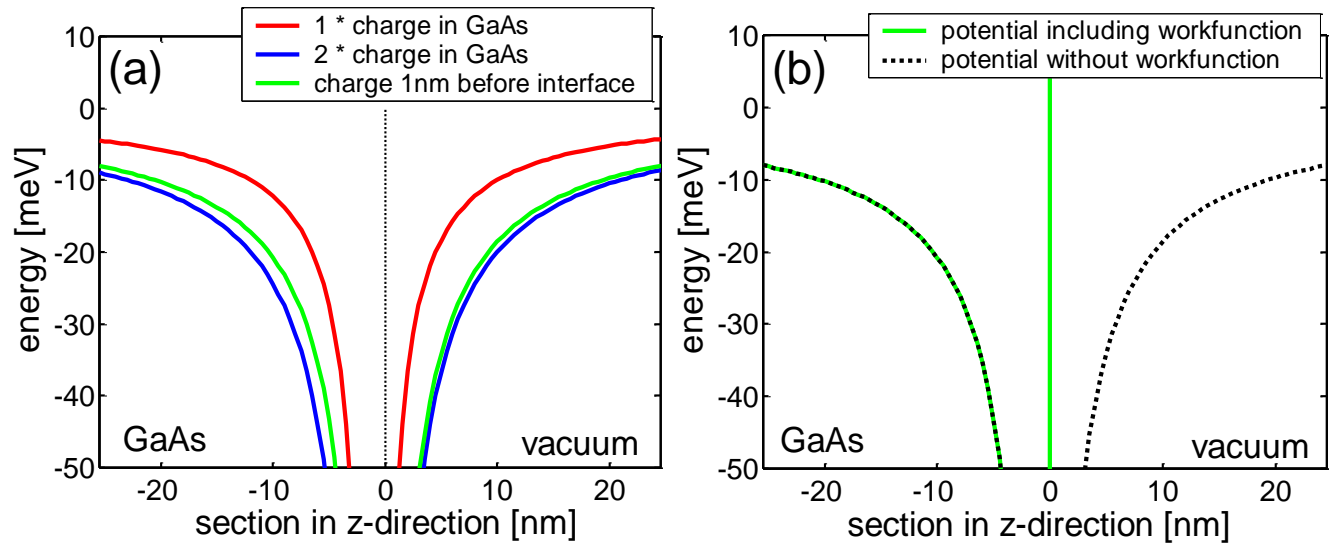

Figure 3.14: For a charge close to an interface there are two competing effects which can change the binding energy. Image (a) illustrates the change of the potential due to the different dielectric constants in GaAs and vacuum. In (b) the cut of the potential due to the work function at the surface is shown.

length of the box by a factor of two would reduce the binding energy by a factor of four.

From this rough estimation one could conclude that the total amount of the binding energy does not change much. Both factors are just obtained by a rough estimation and the shift of the binding energy depends on which effect wins. Our measured data show that the binding energy is enhanced (Figure 3.13(b)), which is a hint that the first effect is the dominant one. 


\subsection{Interacting donors}

After the discussion on single donors in the previous section, this section will focus on the interaction of two donors. It has been shown that the charge configuration of single donors is determined by the position of the tip and the applied gate voltage. In contrast a two donor system shows a more complex behavior. It will be discussed that the electrostatic interaction between the donors in combination with the larger binding energy close to the surface results in ionization gaps (subsection 3.5.1) and bistable charge switching behavior (subsection 3.5.2 and 3.5.3). Uncertainties in the used simulation are summarized in the last subsection 3.5.4. The interaction of charge centers on a different system has been observed in the STM by Nazin et al. [55], they also see ionization gaps.

\subsubsection{Description of the interaction}

Figure 3.15(a) shows a constant current topography of the GaAs(110) surface including two donors visible in the topography image. A $\mathrm{d} I / \mathrm{d} V(x, V)$-section along the yellow solid line running through the dopants' positions is shown in Figure 3.15(b). The dominating bright lines of the $\mathrm{d} I / \mathrm{d} V(x, V)$-signal show the ionization of donors as a function of the tip position and bias voltage.

The ionization occurs along a contour line of a constant value TIBB. The simulation of the TIBB was performed similar to section 3.2. The critical TIBB at each donor position is labeled as $\mathrm{TIBB}_{\mathrm{c} 1}$ and $\mathrm{TIBB}_{\mathrm{c} 2}$ for the donor $\mathrm{D}_{1}$ and the donor $\mathrm{D}_{2}$, respectively. The corresponding position dependent bias voltage is labeled as $V_{\mathrm{c} 1}$ and $V_{\mathrm{c} 2}$, respectively. The minimum of the contour line is centered at the dopant atom, which is schematically shown in Figure 3.15(c) as the blue solid line for $D_{1}$ and the red solid line for $\mathrm{D}_{2}$. The thick black lines represent the measured $\mathrm{d} I / \mathrm{d} V$ signal. If the bias voltage is lower than the solid blue and the solid red curve (i.e. $V<V_{\mathrm{c} 1}, V_{\mathrm{c} 2}$ ) both donors are neutral $\left(\mathrm{D}_{1}{ }^{0}, \mathrm{D}_{2}{ }^{0}\right)$. Keeping the tip close to $\mathrm{D}_{1}$, the donor behaves as an isolated donor and ionizes at TIBB $=\mathrm{TIBB}_{\mathrm{c} 1}$. For TIBB $>\mathrm{TIBB}_{\mathrm{cl}} \mathrm{D}_{1}$ is ionized while $\mathrm{D}_{2}$ is still neutral $\left(\mathrm{D}_{1}^{+}, \mathrm{D}_{2}{ }^{0}\right)$. The reverse situation $\left(\mathrm{D}_{1}{ }^{0}, \mathrm{D}_{2}^{+}\right)$happens when the tip is close to $\mathrm{D}_{2}$.

At a certain point both lines intersect. The experimental data show that those lines do not cross each other unperturbed (e.g. they do not follow the blue and red solid line in Figure 3.15(c), but are continued at a higher voltage with an offset of $0.2 \mathrm{~V}$. This means that in the vicinity of the ionized donor $\mathrm{D}_{1}{ }^{+}\left(\mathrm{D}_{2}{ }^{+}\right)$the ionization curve of $\mathrm{D}_{2}$ $\left(D_{1}\right)$ is shifted to a higher voltage. In Figure 3.15(c) (dotted lines) this is schematically shown as an upwards shift of the contour line of the constant critical value. The charge state as a function of position and voltage is indicated in Figure 3.15(c). The potential landscape, which is the TIBB (black line) superimposed on the Coulomb charge of the donors (green line) is shown schematically in Figure 3.15(d). The charge states of both donors are illustrated by the blue function, which should schematically represent the wave function of the bound electron. The difference in the critical $\mathrm{TIBB}_{\mathrm{c}}$ is due to the different depth of the two donors. 


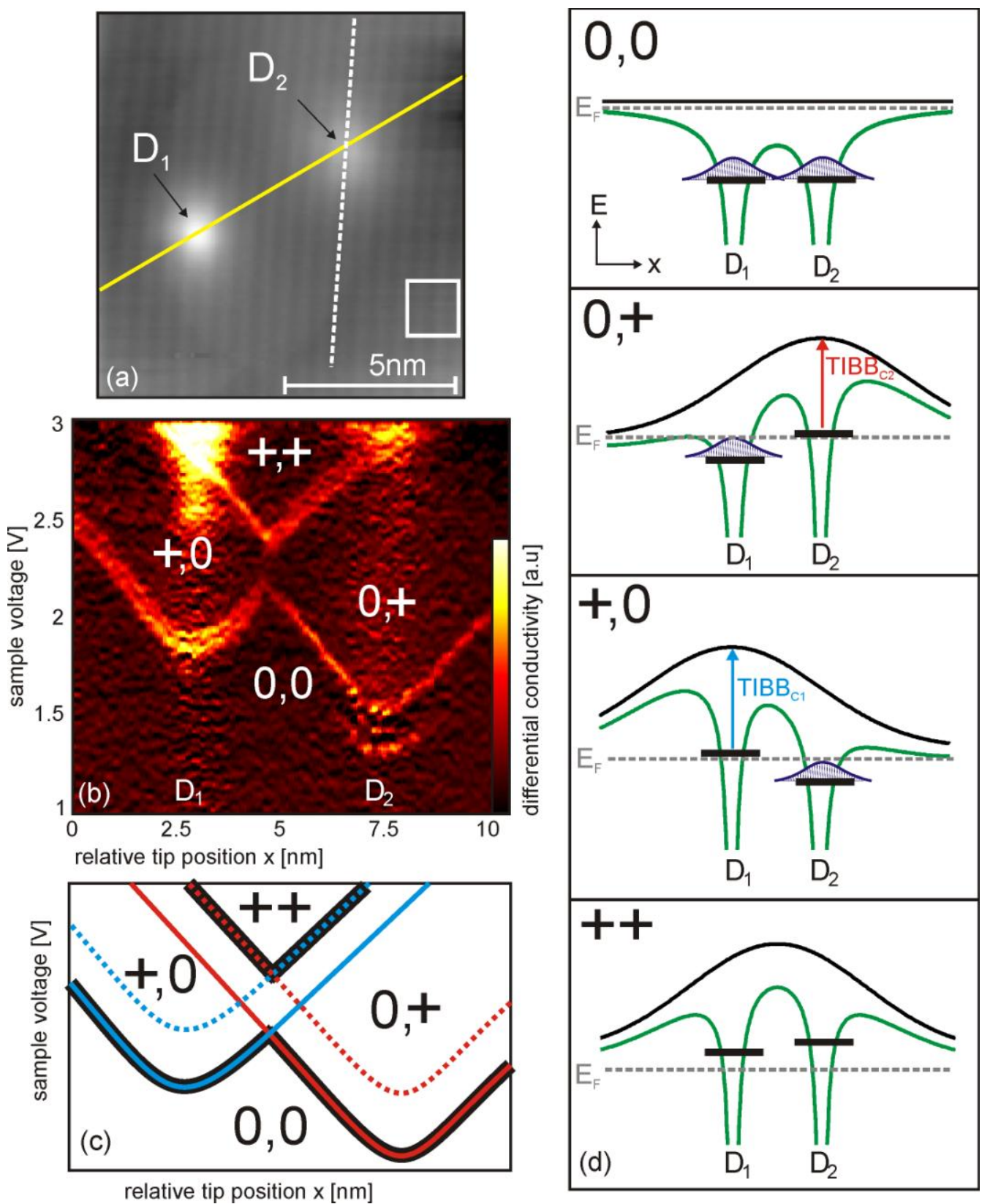

Figure 3.15: (a) constant current topography image taken at a voltage of $3 \mathrm{~V}$ and setpoint current of $0.5 \mathrm{nA}$. (b) $\mathrm{d} / / \mathrm{d} V(x, V)$-section taken along the yellow line. In order to enhance the contrast, an averaged spectrum taken on the free surface (white rectangle) is subtracted. In (c) the neutral (0) and ionized charge states (+) of the two donors are labeled by $00=\mathrm{D}_{1}{ }^{0}, \mathrm{D}_{2}{ }^{0},+0=\mathrm{D}_{1}{ }^{+}, \mathrm{D}_{2}{ }^{0}, 0+=\mathrm{D}_{1}{ }^{0}, \mathrm{D}_{2}{ }^{+}$, and $++=\mathrm{D}_{1}{ }^{+}, \mathrm{D}_{2}{ }^{+}$, respectively. The solid black lines correspond to the observed enhanced differential conductivity (bright lines in b). The solid lines (red and blue) indicate the ionization curves as expected for a single donor; the dotted lines indicate the shifted ionization curve as expected for a donor in the vicinity of a second charged donor. (The white dashed line in (a) marks the $d / / d V(y, V)$-section discussed in Figure 3.23) (d) The potential landscape of this configuration - donor potential superimposed on the TIBB is shown as the green curve. 


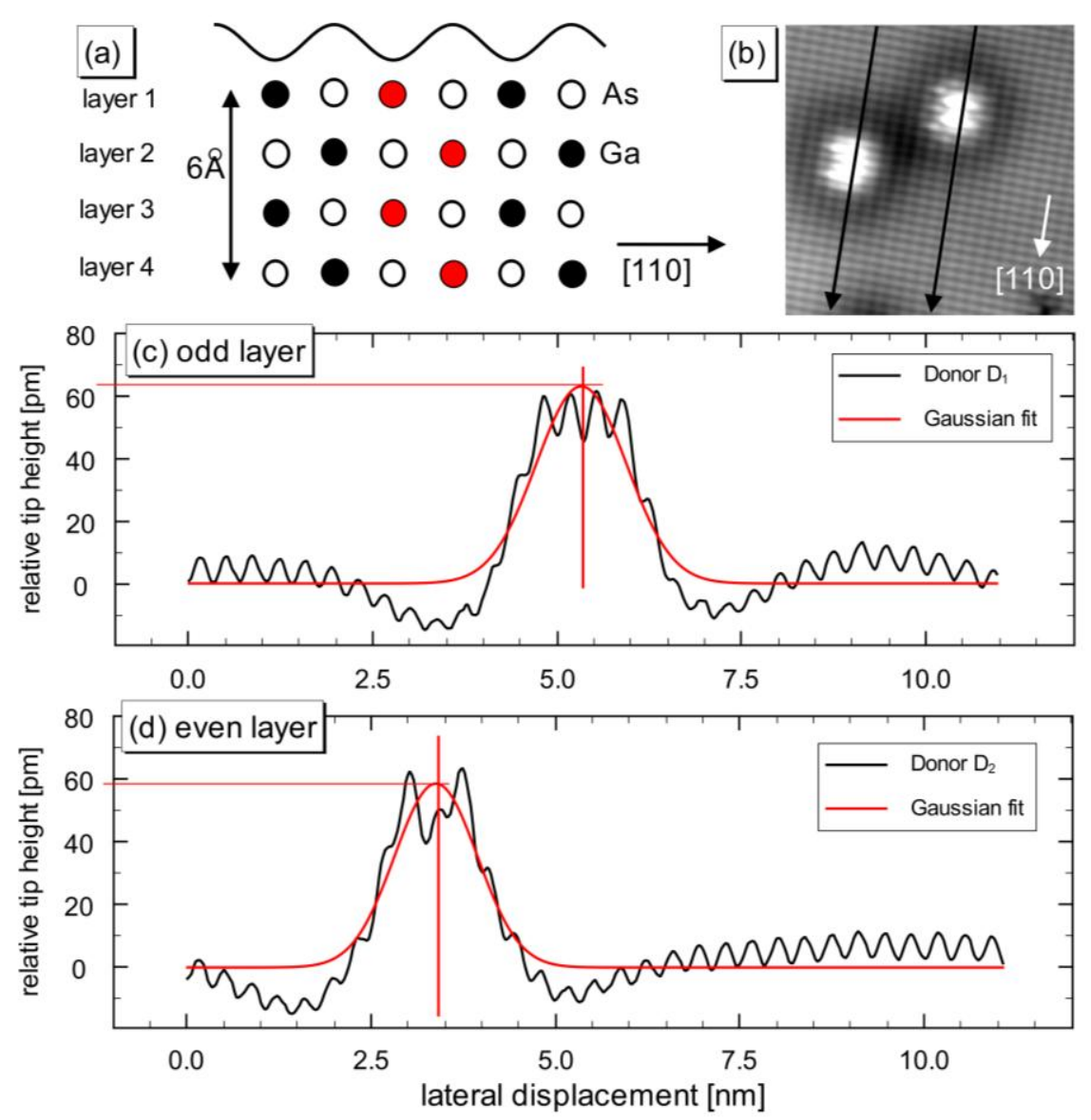

Figure 3.16: Analyzing the layer depth: (a) shows the crystal orientation which is used to determine the layer depth. At negative voltage the atomic corrugation has its maximum at the arsenic site; the position of the donor is on a gallium site (red circles). A topography image of the two donors at $-2 \mathrm{~V}$ and $0.1 \mathrm{nA}$ is shown in (b) $\left(12 \times 12 \mathrm{~nm}^{2}\right)$. For each donor a topography section in [110] direction is shown (black lines) together with a Gaussian fit (red line). The donor $\mathrm{D}_{1}$ is found to be in an odd layer (c) the donor $D_{2}$ is found to be in an even layer (d). From the height of the Gaussian fit one can conclude that the layer of $D_{1}$ is smaller than $D_{2}$.

The different depths are supported by topography image at $-2 \mathrm{~V}$, which is shown in Figure 3.16. Topography cross sections across the two donors in [110] direction are shown. The comparison of the atomic corrugation with the maximum of the donor contrast lead to the conclusion that $\mathrm{D}_{1}$ is in an odd layer and $\mathrm{D}_{2}$ is in an even layer. The height of the Gaussian fit, shows that the layer number of $D_{1}$ is smaller than $D_{2}$ (Analysis according to $[52,53,36]$ ). 


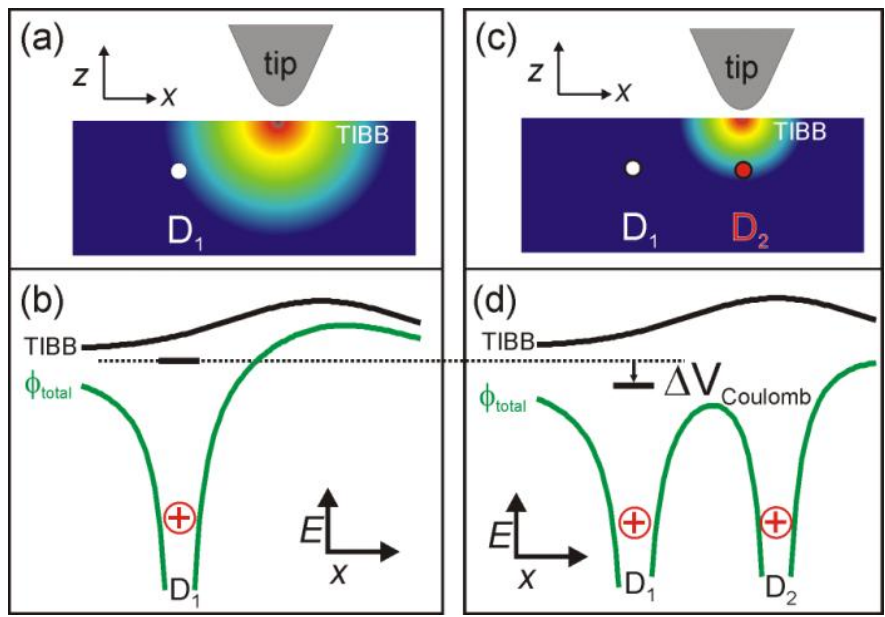

Figure 3.17: The ionization induced by the tip induced band bending (TIBB, colored areas in $(a, c)$ and black lines in $(b, d))$ for an electron bound to $D_{1},(a, b)$ depends on the presence of a second charged donor $D_{2}(c, d)$. The potential landscape of this configuration - charged donors superimposed on the TIBB - is shown as the green curve $(b, d)$. The ionization level is marked by the short black line. The Coulomb potential of $D_{2}$ causes a reduction of the TIBB. This increases the ionization threshold by $\Delta V_{\text {coulomb }}$ with respect to the Fermi energy (black dashed line).

To describe the origin of the ionization gap and to estimate the Coulomb interaction from the measured voltage shift, a single donor is compared with a double donor system (Figure 3.17). While the TIBB is considered as a homogeneous contribution to the potential landscape, the Coulomb potential $V_{\text {Coulomb }}^{D 1}(r)$ of the donor charge is taken into account for one donor (Figure 3.17(a,b)) and for two donors $V_{\text {Coulomb }}^{D 1}(r)+V_{\text {Coulomb }}^{D 2}(r)$ (Figure 3.17(c,d)), respectively. The total potential landscape is described by:

$$
\phi_{\text {total }}(r, V)=\operatorname{TIBB}(r, V)+V_{\text {Coulomb }}^{D 1}(r)+V_{\text {Coulomb }}^{D 2}(r)
$$

To estimate the shift of the potential of $\mathrm{D}_{1}$ by the influence of a second ionized donor a pure electrostatic effect is considered. As a first guess, a constant potential shift $\Delta V_{\text {Coulomb }}$ caused by the potential of $\mathrm{D}_{2}$ at the center of $\mathrm{D}_{1}$ is assumed. Such a model implies that the critical TIBB value of ionization is shifted to:

$$
\mathrm{TIBB}_{c}^{*}=\mathrm{TIBB}_{c}+\Delta V_{\text {Coulomb }}
$$

The shift of the potential can be estimated by using the known Coulomb potential:

$$
\Delta V_{\text {Coulomb }}=Q \cdot\left(4 \pi \varepsilon_{0} \varepsilon_{r} r\right)^{-1}
$$

The experimentally determined distance between $\mathrm{D}_{1}$ and $\mathrm{D}_{2}$ is $r=4.7 \mathrm{~nm}$. The image charge of $\mathrm{D}_{2}$ is taken into account using a dielectric constant $\varepsilon_{\mathrm{r}}=8$ (section 3.2).

For the charge $\mathrm{Q}=1 \mathrm{e}$ we obtain $\Delta V_{\text {Coulomb }}=39 \mathrm{mV}$. In order to compare this value with the measured bias voltage shift of $0.2 \mathrm{~V}$ the TIBB was calculated. The parameters, such as tip geometry, tip-sample-distance, flat band conditions and doping concentration are varied within reasonable limits; details for the simulation can be found in subsection 3.5.4. The difference of the TIBB at $1.8 \mathrm{~V}$ and the TIBB at $2.0 \mathrm{~V}$ at $\mathrm{D}_{1}$ is found to be $\triangle \mathrm{TIBB}=21( \pm 7) \mathrm{meV}$. The denoted uncertainty corresponds to the variation of the parameters for the TIBB calculations. The TIBB value is about a 
factor of two smaller than the estimated value of $\Delta V_{\text {Coulomb. }}$. Both values $\Delta V_{\text {Coulomb }}$ and $\Delta$ TIBB are based on certain assumptions. $\Delta V_{\text {Coulomb }}$ will change significantly if the influence of the surface and the metallic tip on the effective $\varepsilon_{\mathrm{r}}$ is taken into account. The homogeneous simulation of TIBB gives ensemble averaged values which can only serve as a qualitative guideline for the dependence of the TIBB on the position. In addition due to the half space geometry the center of mass of the donor wave function might be shifted into the bulk [39] which could further reduce the overlap between the wave function of the donor and the TIBB. The trend in the data indicates that the experimentally determined shift of the ionization threshold is smaller than the effect of a bare Coulomb potential.

A special configuration occurs directly at the intersection of the ionization curves of the two donors. As soon as one donor ionizes, the second donor level is shifted by $\Delta V_{\text {Coulomb }}$ and thus is forced into the neutral charge state. The outcome of this is two possible stable situations with the same total energy: either $D_{1}$ is ionized and $D_{2}$ is neutral or $\mathrm{D}_{1}$ is neutral and $\mathrm{D}_{2}$ is ionized. The actual charge configuration depends on the history of the build-up process of the space charge region. One may therefore expect hysteretic behavior in such a system. When the tip scans from left to right at a bias voltage between $2.1 \mathrm{~V}$ and $2.3 \mathrm{~V}$ (Figure 3.15a) the result is $\left(\mathrm{D}_{1}{ }^{+}, \mathrm{D}_{2}{ }^{0}\right)$ and when the tip approaches from the right the result it is $\left(\mathrm{D}_{1}{ }^{0}, \mathrm{D}_{2}{ }^{+}\right)$. As the intersection point is approximately located half way between the two donors, the ionization process of each donor causes the same amount of tunneling current enhancement. This means the ionization processes of both donors is indistinguishable and a bistability resulting from an interaction of $\mathrm{D}_{1}{ }^{0}$ and $\mathrm{D}_{2}{ }^{+}$at the intersection of the parabola can in principle not be observed.

\subsubsection{Principle of bistable charge switching}

In this section a donor system is investigated, where both ionization curves are distinguishable. In such configuration a bistability is in principle observable. For this requirement a suitable configuration would be that the distances of the donors'

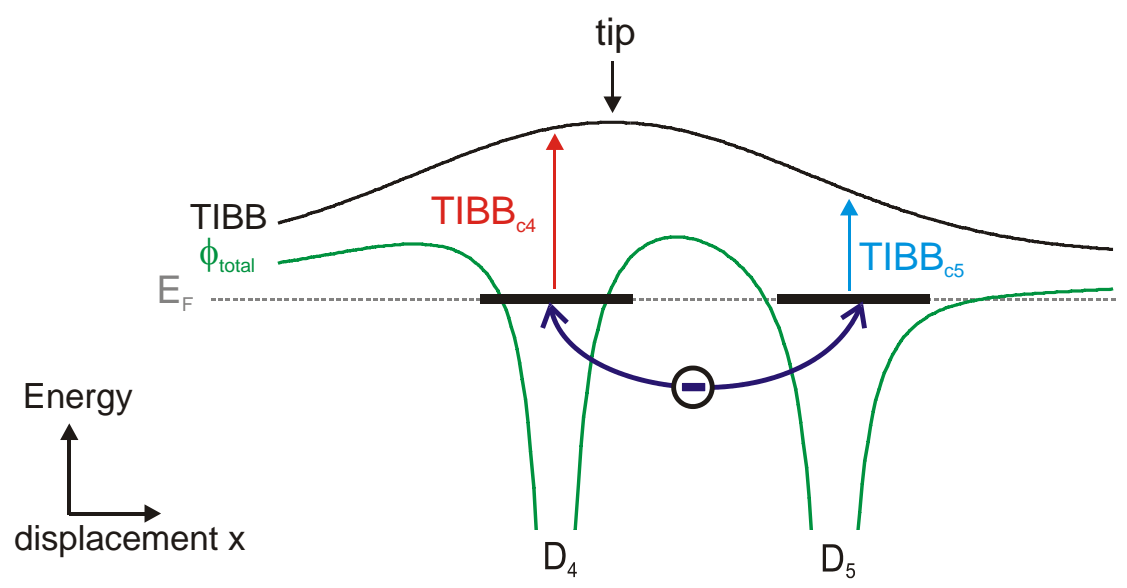

Figure 3.18: Illustration of bistable behavior. The bound states of the donors cross the Fermi-energy at the same voltage. The system can decide to keep the electron at the donor $\mathrm{D}_{4}$ or at the donor $\mathrm{D}_{5}$. The critical amount of $\mathrm{TIBB}_{\mathrm{c} 4}$ and $\mathrm{TIBB}_{\mathrm{c}}$ is different for donors in different subsurface layers. For this situation the tip is close to $D_{4}$ and the impact on the tunnel current is larger for the donor $D_{4}$ than for the donor $D_{5}$. 

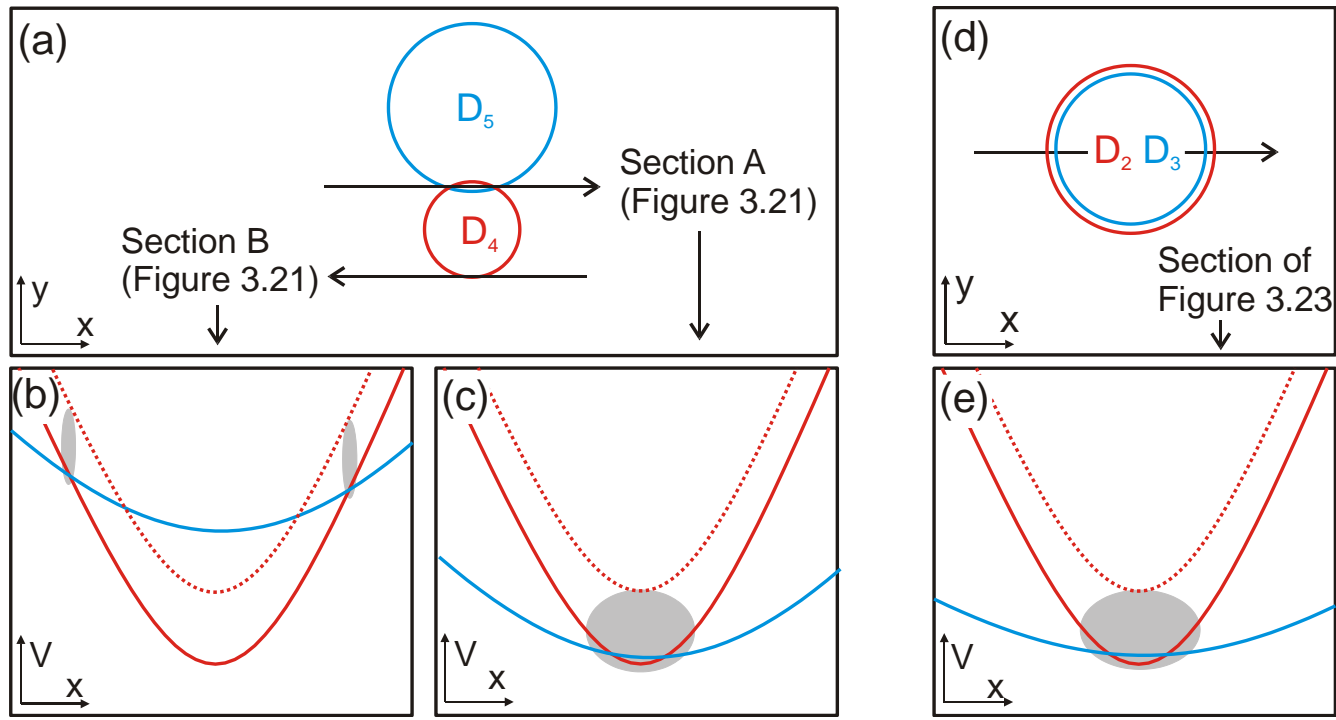

Figure 3.19: Schematic visualization of the expected ionization curves: In (a) and (d) the ionization is shown laterally resolved, for two donors located next to each other (a) and two donors above each other (d). In (b) and (c) the voltage dependent ionization curves for different sections (marked in (a)) are compared. The voltage dependent ionization curve in (e) is similar to the curve in (c). It will be discussed in chapter 3.5.3.

positions to the intersection point has to be different for both donors. If this is the case the amount of tunneling current enhancement at the intersection point is different for each ionization curve.

Figure 3.18 illustrates the case of bistable behavior. The position of the tip is adjusted such that the bound state of $\mathrm{D}_{4}$ and $\mathrm{D}_{5}$ crosses the Fermi energy at the same applied voltage. The critical amount of $\mathrm{TIBB}_{\mathrm{c} 4}$ and $\mathrm{TIBB}_{\mathrm{c} 5}$ to ionize one of these donors is not necessarily the same, as the binding energy for donors in different subsurface layers is not the same (section 3.4). In this situation the distance of the tip to the donor center is different and also the impact on the tunnel current is larger for the donor $\mathrm{D}_{4}$ than for the donor $\mathrm{D}_{5}$. The system can decide to keep the electron at the donor $\mathrm{D}_{4}$ or at the donor $\mathrm{D}_{5}$.

The expected ionization curve for such a donor configuration is schematically shown in Figure 3.19(a-c). In Figure 3.19(a) the ionization is shown laterally resolved. As $\mathrm{D}_{4}$ needs a higher critical $\mathrm{TIBB}_{\mathrm{c} 4}$ its ionization ring at a certain voltage will be smaller than the ionization ring of $\mathrm{D}_{5}$. The expected voltage dependent ionization curves for different sections are shown in Figure 3.19(b) and Figure 3.19(c). Interaction between both donors happens, when both ionization curves are energetically close together. In Figure 3.19(b) this happens in a small lateral area around the intersection point at high voltages (marked grey). In Figure 3.19(c) this happens at the minimum of the ionization curve in a larger lateral area (marked grey). $\mathrm{d} I / \mathrm{d} V(V)$-spectra taken directly at the minimum of the ionization curves in Figure 3.19(b) and Figure 3.19(c) would be directly comparable with each other. The ionization jump for $\mathrm{D}_{4}$ should occur in both cases at the same voltage. The difference is that in the case of Figure 3.19(b) the donor $\mathrm{D}_{4}$ and $\mathrm{D}_{5}$ do not interact with each other (at the minimum of the ionization curve); in the case of Figure 3.19(c) the two donors do interact with each other (at the minimum of the ionization curve). 

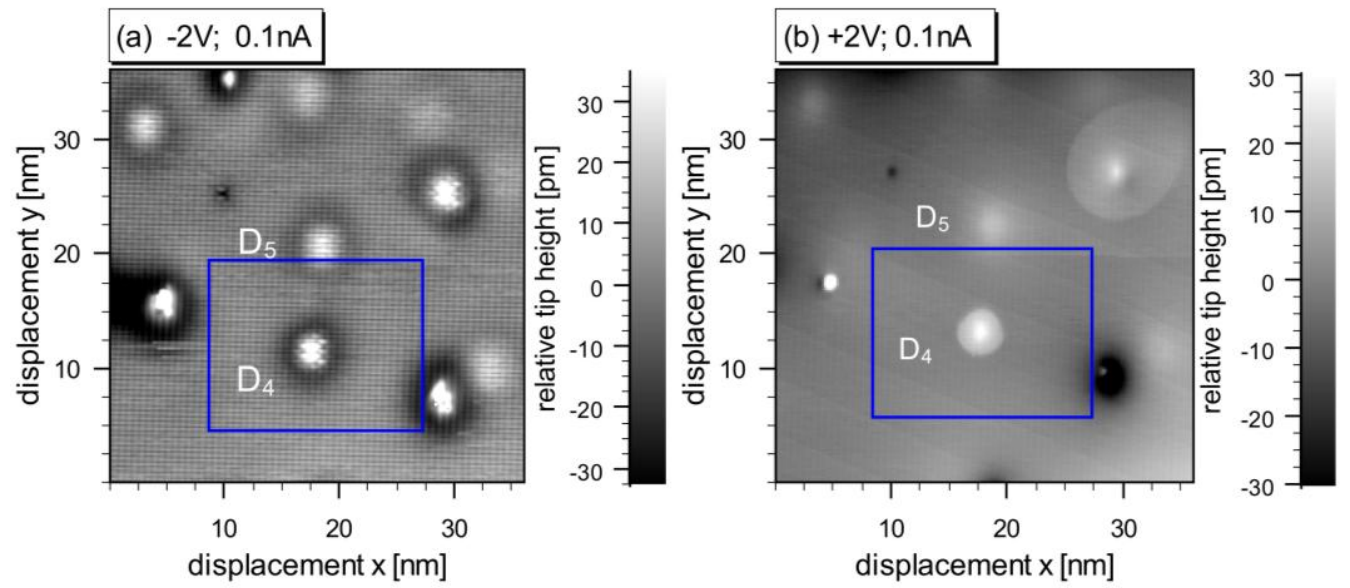

Figure 3.20: Constant current topography images taken at (a) $-2 \mathrm{~V}$ and $(\mathrm{b})+2 \mathrm{~V}$. Two important donors can be identified by their positive contrast for $+2 \mathrm{~V}$ and their Friedel oscillations for $-2 \mathrm{~V}$. The blue box indicates the position of the laterally resolved spectroscopy of Figure 3.21.

In Figure 3.19(d-e) the expected ionization for two donors above each other is visualized. The expected ionization curve in Figure 3.19(e) is similar to the ionization curve in Figure 3.19(c). This donor configuration will be discussed in subsection 3.5.3.

In Figure 3.20 two constant current topographies at a voltage of (a) $-2 \mathrm{~V}$ and (b) $+2 \mathrm{~V}$ are shown. In this image two donors are labeled by $\mathrm{D}_{4}$ and $\mathrm{D}_{5}$. The contrasts at negative voltages show the well known Friedel oscillations, and due to their height difference, one can identify that $\mathrm{D}_{4}$ is closer to the surface than $\mathrm{D}_{5}$. This observation is confirmed by the contrast at positive voltage, the ionization ring for $\mathrm{D}_{5}$ is larger than the ring for $\mathrm{D}_{4}$. The blue box indicates the position of the laterally resolved spectroscopy shown in Figure 3.21. Here $\mathrm{d} I / \mathrm{d} V(x, y)$-maps for different voltages can be seen in Figure 3.21(a-d). An averaged spectrum was subtracted from the $I(V)$-spectra. The data are shown as raw data; no current averaging has been done after the measurement. At $1.22 \mathrm{~V}$ (Figure 3.21(a)) the ring of donor $\mathrm{D}_{5}$ is clearly visible; as a guide to the eye the ring is indicated by two arrows. At 1.46V (Figure 3.21(b)) the size of the ring has increased, but its intensity has decreased as the ring is more far away from the donor center [41]. At the same voltage the ring of donor $\mathrm{D}_{4}$ has started. At $1.64 \mathrm{~V}$ (Figure 3.21(c)) the two rings of donor $\mathrm{D}_{4}$ and $\mathrm{D}_{5}$ cross each other (the ring of donor $\mathrm{D}_{5}$ is hardly visible, but still observable and indicated by the two arrows). For a voltage of $1.9 \mathrm{~V}$ (Figure $3.21(\mathrm{~d})$ ) the ring of $\mathrm{D}_{5}$ interacts in the middle of the ring of $\mathrm{D}_{4}$. 

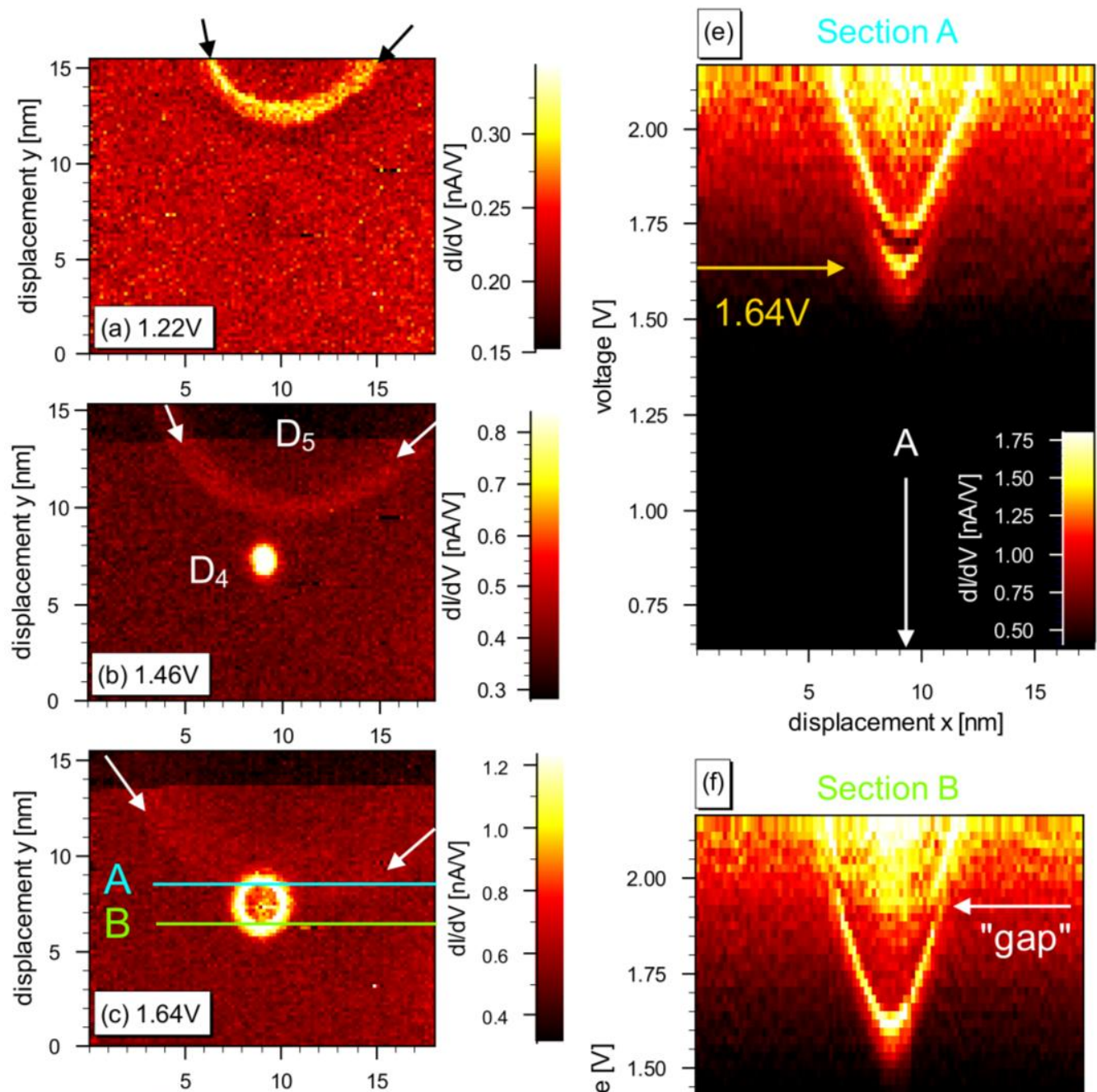

displacement x [nm]
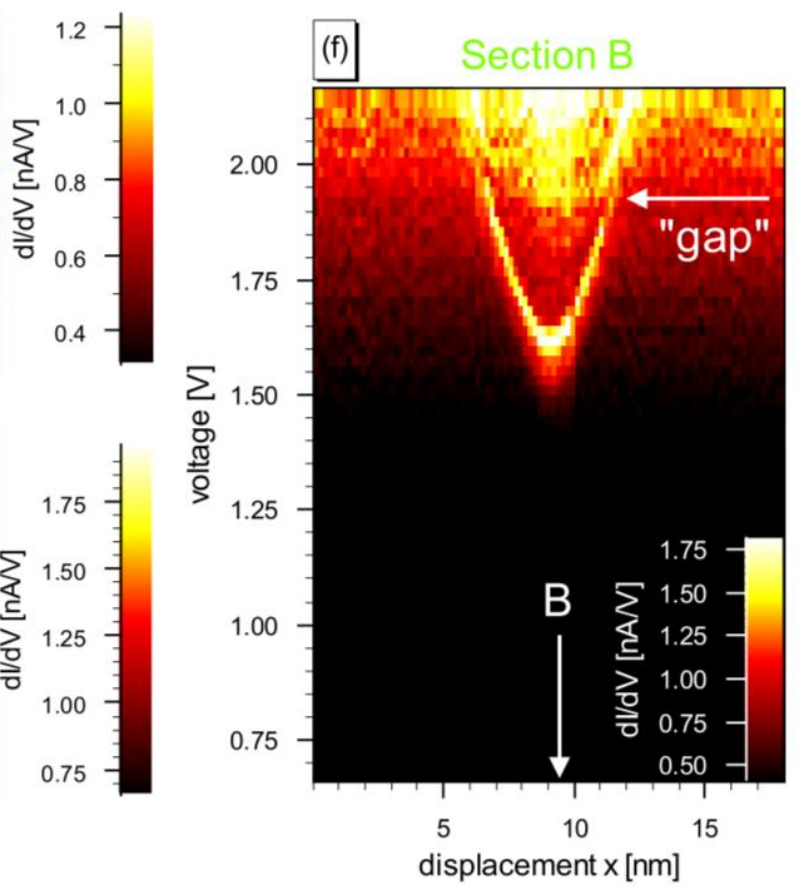

Figure 3.21: (a-d) Laterally resolved $d / / d V(x, y)$-maps for different voltages. (e,f): $\mathrm{d} / / \mathrm{d} V(x, V)$-sections taken at the position indicated with the blue and green line at the $\mathrm{d} / / \mathrm{d} V(x, y)$-map of $1.64 \mathrm{~V}$.

Two $\mathrm{d} I / \mathrm{d} V(x, V)$-sections Figure 3.21(e,f) taken at two sections A and B are compared with each other. Their ionization curves can be compared to Figure 3.19(b,c), note that the ionization curve of $\mathrm{D}_{5}$ is not visible. In section A (Figure 3.21(e)) the ring of donor $\mathrm{D}_{5}$ crosses donor $\mathrm{D}_{4}$ directly at the minimum. At $1.64 \mathrm{~V}$ one can see that the parabola is shifted to higher voltages, but what is also important; the parabola does not vanish for the lower voltages. 

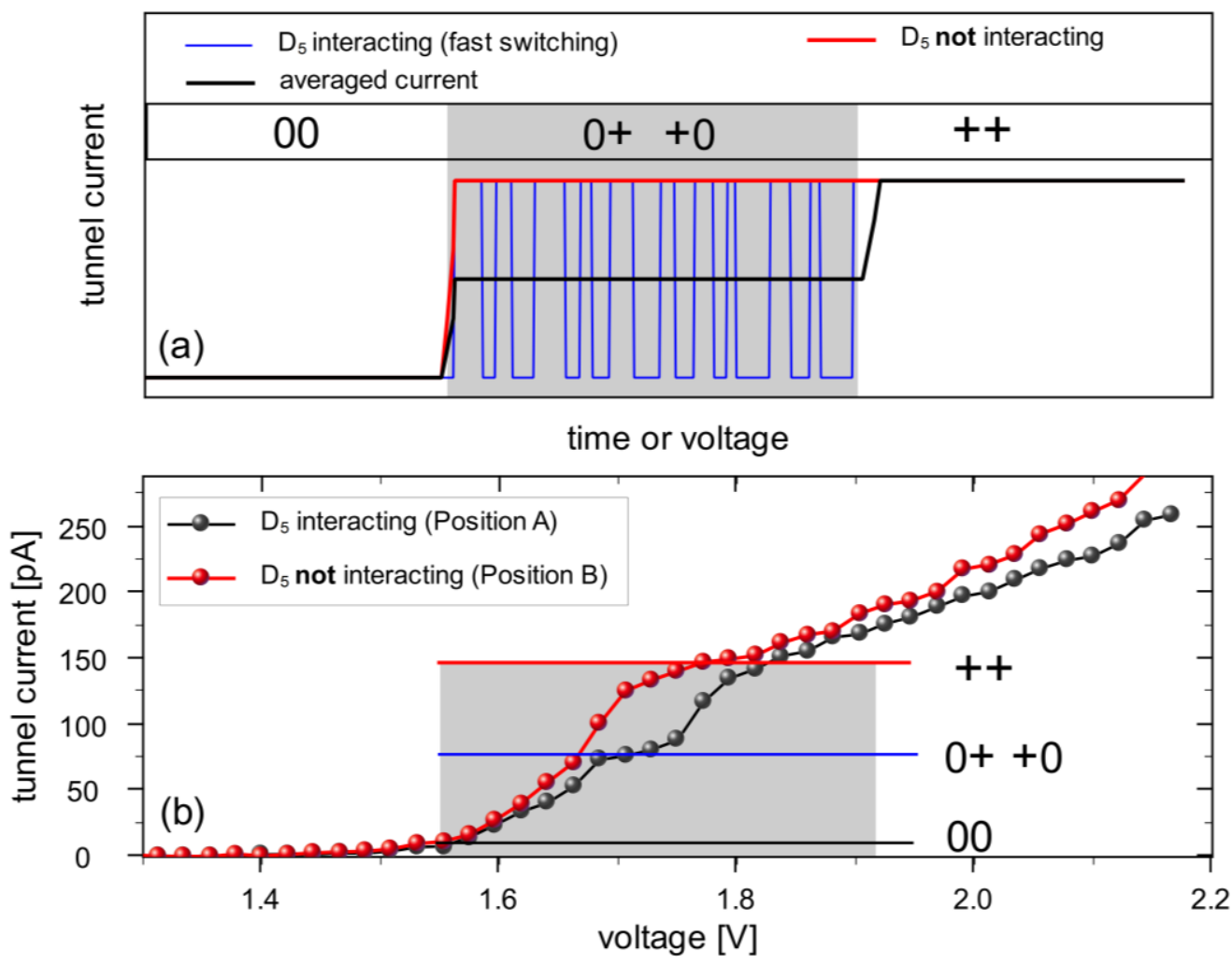

Figure 3.22: In (a) the expected tunnel current is shown. At a position where $D_{5}$ interacts with $D_{4}$ one would expect a switching like the blue line. If this is faster than the STM's resolution one will measure the average, which is the black line. At a position where $D_{5}$ does not interact with the $D_{4}$ one would expect the red curve for $D_{4}$. In (b) the measured data are shown for a spectrum at position $A$ (black) and for a spectrum at position B (red) (Figure 3.21).

This would be the case if the interaction would be just a pure Coulomb shift like for the parabola of the donors $D_{1}$ and $D_{2}$ in Figure 3.15(b). Instead the system is bistable. In section $\mathrm{B}$ (Figure 3.21(f)) the ring of donor $\mathrm{D}_{5}$ crosses donor $\mathrm{D}_{4}$ at a higher voltage, indicated with "gap" in the image.

Figure 3.22(a) visualizes the expected $I(V)$-curves for the position A, where the two donors interact and for position $\mathrm{B}$, where the two donors do not interact. For low voltages both donors are neutral $\left(\mathrm{D}_{4}{ }^{0} \mathrm{D}_{5}{ }^{0}=00\right)$. For high voltages both donors are ionized $\left(\mathrm{D}_{4}{ }^{+} \mathrm{D}_{5}{ }^{+}=++\right)$, the additional current occurs due to the ionized donor $\mathrm{D}_{4}$. The blue curve shows the expected current if the system is bistable, then two configurations alternate. The first configurations is that $\mathrm{D}_{4}$ is ionized and $\mathrm{D}_{5}$ is neutral $\left(D_{4}{ }^{+} D_{5}{ }^{0}=+0\right)$ and the current would be high. The second one is that $D_{4}$ is neutral and $\mathrm{D}_{5}$ is ionized $\left(\mathrm{D}_{4}{ }^{0} \mathrm{D}_{5}{ }^{+}=0+\right)$, than the current would be low. If the switching is faster than the time resolution of the STM, one would obtain an averaged current, indicated by the black line. The bistable behavior ends when the applied voltage is large enough that both donors can be ionized. The red curve shows the expected tunnel current if $D_{5}$ is not interacting with $\mathrm{D}_{4}$. In the experiment, this is the case for the position $\mathrm{B}$ of Figure 3.21(f), where the two ionization rings have not yet crossed each other. Figure 3.22(b) shows two $I(V)$-spectra, one is taken at position A, where both donors interact (black line), and on is taken at position B, where they do not interact (red line). For the 
interacting curve two jumps are visible. Between the two jumps there is a plateau which is in good agreement with the expected averaged tunnel current. (This characteristic could be verified by different spectra in the area of bistable behavior in the same data set, as well as from further data sets with different applied voltages). From this measurement one can conclude that bistable behavior is possible.

\subsubsection{Resolving bistable charge switching}

In subsection 3.5.2 it was discussed that bistable charge switching for a double donor system is possible. The switching process was too fast to resolve it with the used STM setup. In this subsection a donor system is investigated, where the switching process between the two donors is slow enough. Two donors which are located above each other are discussed. The expected ionization curves are already schematically visualized in Figure 3.19(d,e).

Figure 3.23(a) shows a laterally resolved $I(y, V)$-section along the white dashed line in the constant current topography in Figure 3.15(a). To enhance the contrast, an averaged spectrum measured on the free surface was subtracted from the data (white rectangle Figure 3.15(a)). In a very narrow region $\left(\sim\right.$ a few $\left.\mathrm{nm}^{2}\right)$ around the donor $\mathrm{D}_{2}$, the current onset characterizing the ionization process starts randomly either at the dominant ionization curve or at a $\sim 220 \mathrm{mV}$ lower voltage or is even changing during the bias voltage sweep. Instability of the tips state is excluded as a second laterally resolved spectroscopy measurement taken at the same position showed the same instability at the same voltage interval and position. Figure 3.23(b) shows a selection of individual current traces taken in the region marked by the white box in Figure 3.23(a). While measuring the $I(V)$-curve the voltage was ramped from high to low values. The average time acquiring a single current data point was $0.2 \mathrm{~ms}$. The tunnel current is plotted versus voltage (top axis), and additionally a timescale indication the time to acquire a spectrum is shown (bottom axis).

With the knowledge of subsection 3.5.2 where two visible donors $\mathrm{D}_{4}$ and $\mathrm{D}_{5}$ interact, this experimental observation can be readily explained by the presence of an additional donor $\mathrm{D}_{3}$ located several nanometers below $\mathrm{D}_{2}$. This scenario is illustrated in Figure 3.25(a), where the potential landscape perpendicular to the surface is shown. The two insets show the voltage dependent TIBB at the position close to the surface (red curve) and the position of about $8 \mathrm{~nm}$ in the surface (blue curve). The TIBB at the position $\mathrm{D}_{3}$ is smaller than the TIBB at the position $\mathrm{D}_{2}$. In combination with an enhanced binding energy for $\mathrm{D}_{2}$ in comparison to $\mathrm{D}_{3}$ (section 3.4) donors which are in different depths below the surface can have donor levels which cross the Fermi energy at the same applied bias voltage making a bistable behavior possible.

Similar to the two donors $\mathrm{D}_{4}$ and $\mathrm{D}_{5}$ in subsection 3.5.2, the impact on the tunnel current is different for two donors above each other ( $\mathrm{z}$ direction). $\mathrm{D}_{3}$ is too far away from the surface to have a direct impact on the tunnel current: Its charge state is only visible by the shift of the ionization curve of $D_{2}$. Thus, the ionization state of $D_{2}$ and $\mathrm{D}_{3}$ are distinguishable. A shift occurs due to Coulomb interaction, similar to the case of laterally neighboring donors, as discussed in subsection 3.5.1. 

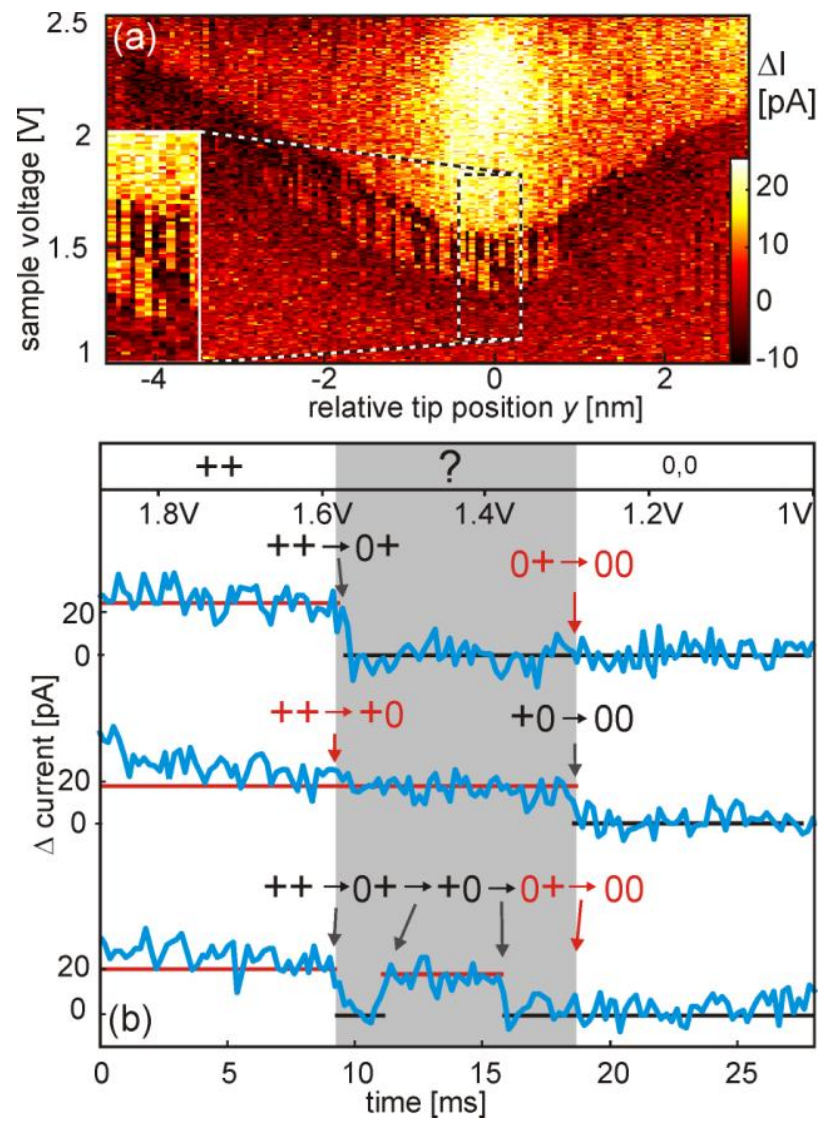

Figure 3.23: A laterally resolved $I(y, V)$-section along the white dashed line in Figure $3.15(a)$ is shown in (a). In (b) a selection of single $I(V)$-spectra taken in the vicinity of the $D_{2}$ show the switching process in the bistable region. As a guide to the eye the high current configuration $\left(D_{2}\right.$ is ionized) are marked by red lines, the low current one ( $D_{2}$ is neutral) by black lines.

In Figure 3.23(b) the charge switching of $\mathrm{D}_{2}$ is seen as jumps in the current trace, they are marked with black labels. A switching of $\mathrm{D}_{3}$ (indicated by red labels in Figure 3.23(b)) solely is not visible in the current trace (see above), but can be identified because it modifies the onset voltage of $\mathrm{D}_{2}$.

A given charge configuration can be stable for more than $10 \mathrm{~ms}$. The switching process itself happens on a timescale faster than the resolution of the STM. Note that for this donor system no averaging of the two configurations during a single trace is observed. Trace 1 shows an example that $\mathrm{D}_{2}$ switches first and stays neutral until $\mathrm{D}_{3}$ becomes neutral. Trace 2 demonstrates the reverse switching order. Bistable behavior is observed as multiple switches between the two configurations as shown in the last trace in Figure 3.23(b) and the inset of Figure 3.23(a). This observation resembles telegraph noise (which is related to burst or popcorn noise) that occurs in semiconductors where random trapping and release of charge carriers are discussed as possible candidates for a bistable behavior [56]:

According to Hsu et al. [56], the average time $\tau$ how long a trap is empty of an electron can be expressed by the electron capture cross section $\sigma$, the thermal velocity of free carriers $v_{t h}$, the effective density of states in the conduction band $N_{C}$, the activation energy $E_{a}$, as well as the temperature $T$ by the following equation: 


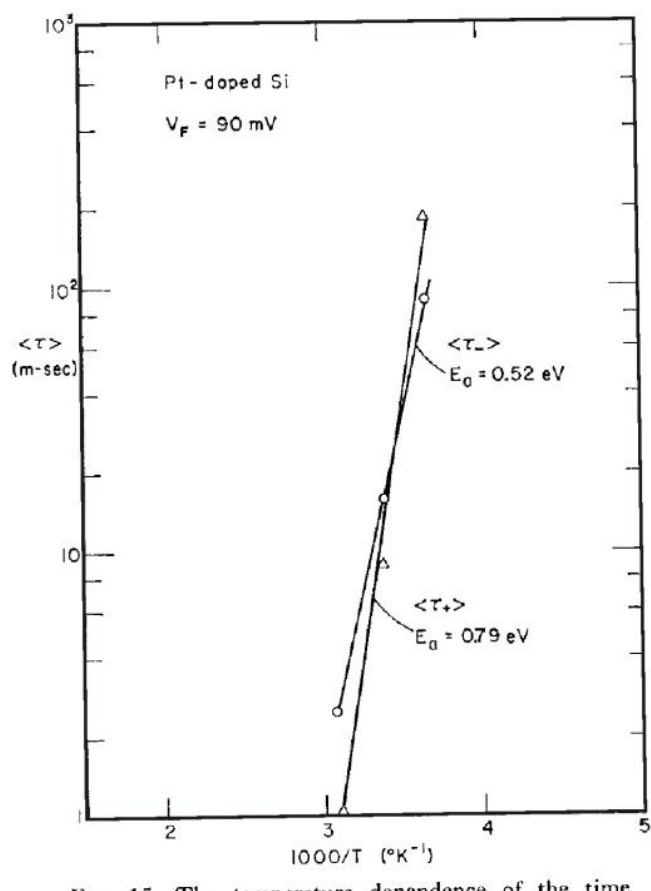

Fig. 15. The temperature dependence of the time constants for a Pt doped diode.

Figure 3.24: Measured burst noise time constant $\tau_{+}$(empty trap, high current) and $\tau$. (filled trap, low current) for a Pt doped silicon pn-diode as a function of temperature. At room temperature the time constants are in the order of $10 \mathrm{~ms}$ for an activation energy of 0.52 and $0.79 \mathrm{eV}[56]$

$$
\frac{1}{\tau}=\sigma v_{t h} N_{C} \exp \left(\frac{E_{a}}{k_{B} T}\right)
$$

The authors investigated silicon devices and extracted the activation energy $E_{a}$ by temperature dependent measurements of the average time $\tau$ (Figure 3.24). It is $0.52 \mathrm{eV}$ for an empty trap $\tau_{+}$(and $0.79 \mathrm{eV}$ for containing an electron $\tau_{-}$). The average time in the order of $\sim 10 \mathrm{~ms}$ is obtained for room temperature. Assuming the product of $\sigma v_{t h}$ and $N_{C}$ as nearly similar in GaAs and $\mathrm{Si}$, only the term in the exponential function is relevant for the time constant. The quotient of the activation energy of $0.52 \mathrm{eV}$ and the temperature of $300 \mathrm{~K}$ found by Hsu et al. is the same like the quotient of an activation energy of $8 \mathrm{meV}$ ( donor binding energy) and a temperature of $5 \mathrm{~K}$ used in our measurements. According to this a time constant in the order of milliseconds is reasonable.

As it is a very rough estimation that the values of $\sigma v_{t h}$ and $N_{C}$ are nearly the same for GaAs and $\mathrm{Si}$, the estimation is done in a different manner. For the values of $v_{t h}$ and $N_{C}$ the parameters of GaAs can be used, here the effective mass and the temperature change the values. Hsu et al. found for their system a cross section of $\sigma=1.5 \cdot 10^{-16} \mathrm{~cm}^{2}$. For GaAs and the above mentioned values, a cross section of $\sigma=2.2 \cdot 10^{-14} \mathrm{~cm}^{2}$ is found. Note that this estimation is not meant to give an exact value for the cross section, as the amount of data is far too little. Instead it should be regarded as a cross check, if the measured time scale could be reasonable. 

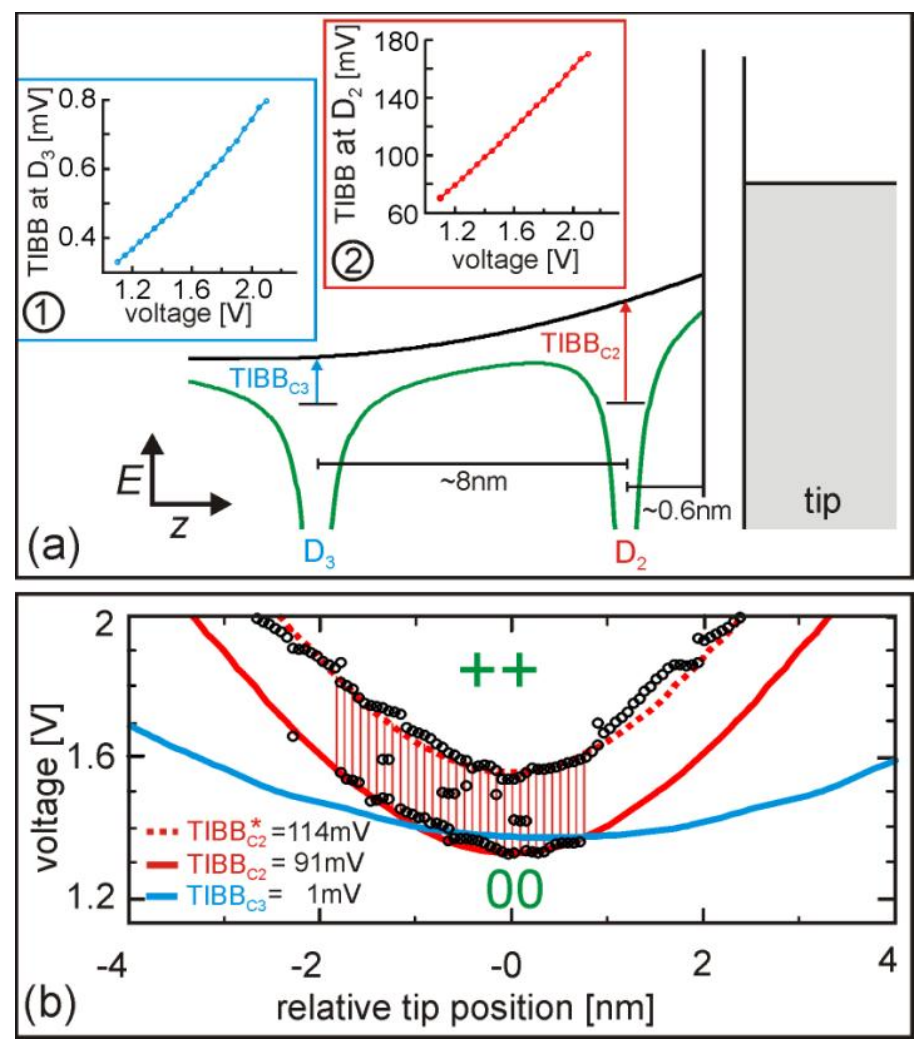

Figure 3.25: Figure (a) shows a schematically view of the potential landscape perpendicular to the surface of two donors plus the potential induced by the tip. It should illustrate the idea that a third donor $D_{3}$ is located below the donor $D_{2}$. The tip induced band bending is lower at the position of the donor $D_{3}$ than for the position of the donor $D_{2}$ as seen in the inset (1) and (2). Most important is that the binding energy of a donor increases towards the surface which implies that a lower value of TIBB is needed for the donor $D_{3}$ to be ionized. Thus, for an appropriate distance both donors can ionize at the same applied bias voltage. A simulation of the voltage dependent TIBB is done for both positions. The contour lines adjusted to the measured voltage jumps from Figure 3.23(a) (black circles) are shown.

The Coulomb interaction was extracted in a similar way as described in subsection 3.5.1. In Figure 3.25(b) the measured switching points extracted from Figure 3.23(a), are compared with the simulated contour line of constant TIBB. The best fit for the lower ionization curve of donor $\mathrm{D}_{2}$ is found with a depth of $0.6 \mathrm{~nm}$ and with a critical value of $\mathrm{TIBB}_{\mathrm{c} 2}=91 \mathrm{mV}$ (solid red line). By using equation (3.7) the shifted ionization curve (dotted red line) corresponds to a contour line of: $\operatorname{TIBB}_{\mathrm{c} 2}{ }^{*}=$ $\mathrm{TIBB}_{\mathrm{c} 2}+\Delta V_{\text {Coulomb }}=114 \mathrm{mV}$. By using equation (3.8) the ionization of $\mathrm{D}_{3}$ causes a shift of $\Delta V_{\text {Coulomb }}=23 \mathrm{mV}$. This value corresponds to a distance of $\sim 8 \mathrm{~nm}$ between $\mathrm{D}_{2}$ and $\mathrm{D}_{3}$. The blue solid line in Figure 3.25(b) shows the ionization curve corresponding to this depth and a slight lateral offset $(0.4 \mathrm{~nm}$ to positive y-direction) of the donor center to the side of $D_{2}$. We conclude that $D_{3}$ is not located directly underneath $D_{2}$. In order to calculate the TIBB contour of $\mathrm{D}_{3}$, we used the same parameter as for the calculations for $\mathrm{D}_{1}$ and $\mathrm{D}_{2}$. The contour line $\mathrm{TIBB}_{\mathrm{c} 3}$ which is $8 \mathrm{~nm}$ below the surface and coincides with the contour line of $\mathrm{TIBB}_{\mathrm{c} 2}$ yields $\mathrm{TIBB}_{\mathrm{c} 3} \sim 1 \mathrm{mV}$. This is on the order of the thermal fluctuations. However, the experiment clearly shows well defined charge states of $D_{3}$. 
For voltages lower than the solid red and solid blue line both $\mathrm{D}_{2}$ and $\mathrm{D}_{3}$ are neutral $\left(\mathrm{D}_{2}{ }^{0}, \mathrm{D}_{3}{ }^{0}\right)$. If the voltage is higher than the solid blue line $\mathrm{D}_{3}$ is ionized $\left(\mathrm{D}_{2}{ }^{0}, \mathrm{D}_{3}{ }^{+}\right)$and the ionization curve for $\mathrm{D}_{2}$ is shifted to higher voltages (dotted red line). For voltages higher than the dotted red line both donors are ionized $\left(\mathrm{D}_{2}{ }^{+}, \mathrm{D}_{3}{ }^{+}\right)$. Between the two states $\left(\mathrm{D}_{2}{ }^{0}, \mathrm{D}_{3}{ }^{0}\right)$ and $\left(\mathrm{D}_{2}{ }^{+}, \mathrm{D}_{3}{ }^{+}\right)$there is an area where the system is bistable (hatched area). Here the solid red and the solid blue line coincide within the thermal broadening. This means that the critical TIBB values $\mathrm{TIBB}_{\mathrm{c} 2}$ at $\mathrm{D}_{2}$ and $\mathrm{TIBB}_{\mathrm{c} 3}$ at $\mathrm{D}_{3}$, are achieved at the same applied voltage. The region of bistability in the experimental data of Figure 3.23(a) is in reasonable agreement with the thermal fluctuations at $5 \mathrm{~K}$.

\subsubsection{Analysis of error for the simulation}

In the previous section a TIBB simulation was used to validate the assumption that bistable behavior between two interacting donors is possible. This subsection should shortly summarize the analysis, which has lead to the denoted errors in the simulation. In the simulation parameters are used, which are not directly detectable during the measurement, and which are only known approximately. These parameters are the flat band condition, the tip-sample distance and the tip radius. Also the donor distance $\mathrm{D}_{2}$ is not exactly known, and is varied in the simulation to the surface, the same is valid for the doping concentration. In the next pages the contour lines by varying these parameters are compared to the data points. In the table next to the image, the values of the contour lines for $\mathrm{TIBB}_{\mathrm{c} 2}, \mathrm{TIBB}_{\mathrm{c} 2}{ }^{*}$ and $\mathrm{TIBB}_{\mathrm{c} 3}$ are summarized, the calculated value $\Delta V_{\text {Coulomb }}=\mathrm{TIBB}_{\mathrm{c} 2}{ }^{*}-\mathrm{TIBB}_{\mathrm{c} 2}$ is listed as well. The values are in the range from $19 \mathrm{mV}$ to $28 \mathrm{mV}$ which corresponds to a distance between donor $\mathrm{D}_{2}$ and $\mathrm{D}_{3}$ of $10 \mathrm{~nm}$ to $6 \mathrm{~nm}$.
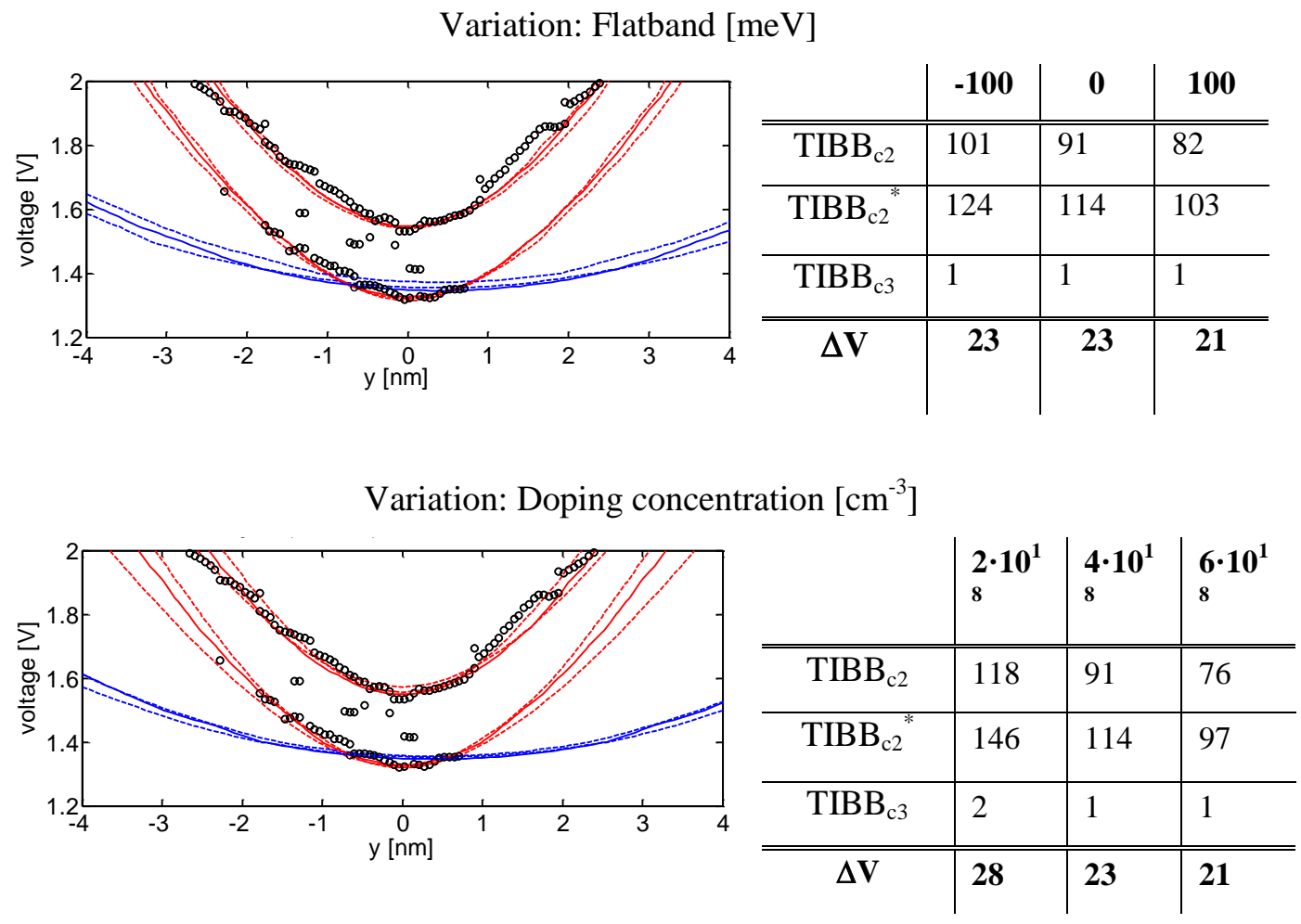
Variation: tip-sample distance [nm]

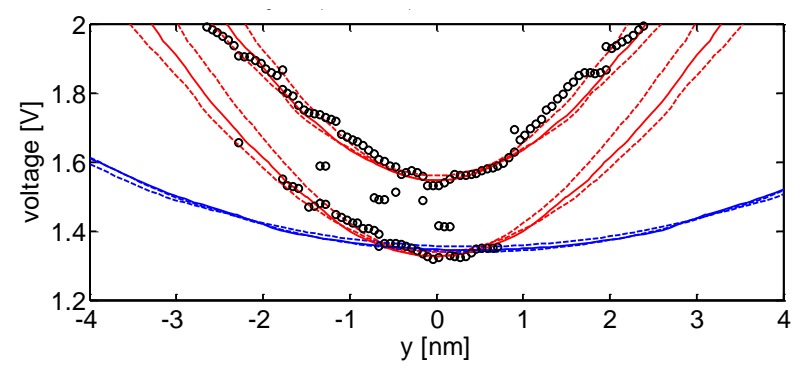

\begin{tabular}{c|l|l|l} 
& $\mathbf{0 . 6}$ & $\mathbf{0 . 8}$ & $\mathbf{1 . 0}$ \\
\hline \hline TIBB 2 & 116 & 91 & 76 \\
\hline TIBB $_{\mathrm{c} 2}{ }^{*}$ & 142 & 114 & 95 \\
\hline TIBB $_{\mathrm{c} 3}$ & 1 & 1 & 1 \\
\hline \hline $\boldsymbol{\Delta V}$ & $\mathbf{2 6}$ & $\mathbf{2 3}$ & $\mathbf{1 9}$
\end{tabular}

Variation: tip radius $[\mathrm{nm}]$

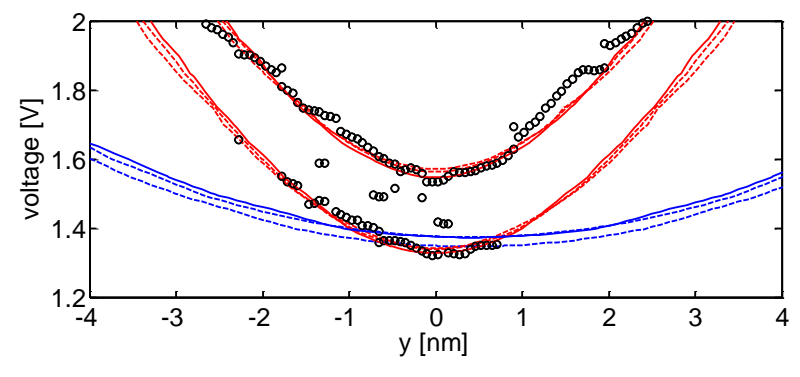

\begin{tabular}{c|l|l|l} 
& $\mathbf{2}$ & $\mathbf{2 . 5}$ & $\mathbf{3}$ \\
\hline $\mathrm{TIBB}_{\mathrm{c} 2}$ & 91 & 102 & 111 \\
\hline $\mathrm{TIBB}_{\mathrm{c} 2}{ }^{*}$ & 114 & 127 & 138 \\
\hline $\mathrm{TIBB}_{\mathrm{c} 3}$ & 1 & 1 & 1 \\
\hline \hline $\boldsymbol{V} \mathbf{V}$ & $\mathbf{2 3}$ & $\mathbf{2 5}$ & $\mathbf{2 7}$
\end{tabular}

Variation: distance of donor $\mathrm{D}_{2}[\mathrm{~nm}]$

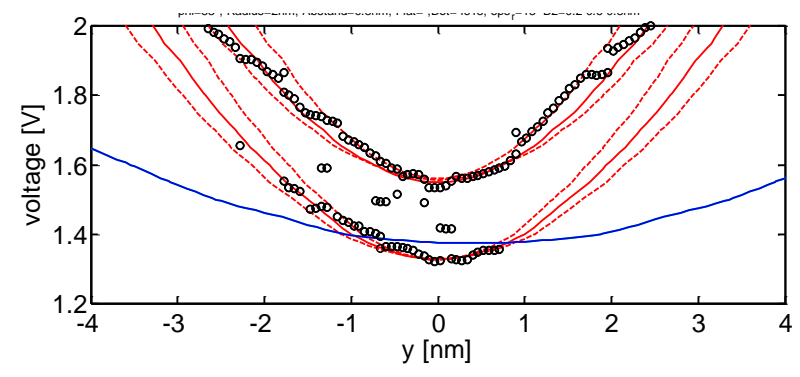

\begin{tabular}{c|l|l|l} 
& $\mathbf{0 . 2}$ & $\mathbf{0 . 6}$ & $\mathbf{1}$ \\
\hline \hline TIBB $_{\mathrm{c} 2}$ & 119 & 91 & 71 \\
\hline TIBB $_{\mathrm{c} 2}{ }^{*}$ & 147 & 114 & 90 \\
\hline TIBB $_{\mathrm{c} 3}$ & 1 & 1 & 1 \\
\hline \hline $\boldsymbol{V} \mathbf{V}$ & $\mathbf{2 8}$ & $\mathbf{2 3}$ & $\mathbf{1 9}$
\end{tabular}

Variation: distance of donor $\mathrm{D}_{3}[\mathrm{~nm}]$

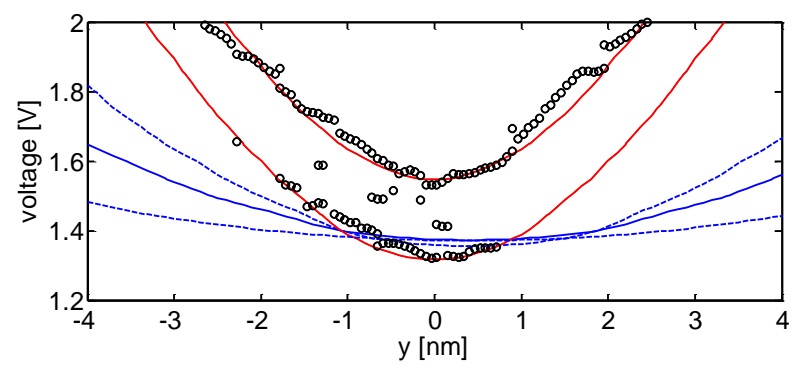

\begin{tabular}{l|l|l|l} 
& $\mathbf{5}$ & $\mathbf{8}$ & $\mathbf{1 2}$ \\
\hline \hline TIBB $_{\mathrm{c} 3}$ & 3 & 1 & 0.5
\end{tabular}

Figure 3.26: Variation of the TIBB simulation parameter. 


\subsection{Beyond the model}

The donor systems investigated in the previous section 3.5 have a distance of about $5 \mathrm{~nm}$, they were described as independent and the interaction is only included by Coulomb interaction. In comparison with the Bohr radius of the bulk effective mass donor of $10 \mathrm{~nm}$ ( $\mathrm{Si}$ in GaAs) a simple superposition of two independent donors is a very simplified description of the system. Instead the two donor system should have to be treated like a molecule (see for example $[50,57]$ ).

Two ionized donors are two positive charges. They are embedded with a fixed distance in the host material and their distance will not change by introducing an electron in the potential. The potential energy of the two positive charges will thus stay constant during the charging process.

By occupying the two positive charges with an electron the system is comparable to the hydrogen-molecule ion $\mathrm{H}_{2}{ }^{+}$having a fixed distance of the two ions. The two energetically lowest solution of the system are the bound and the antibound state, the bound state is energetically lower than the antibound state. The states have in common that the electron density is symmetrically distributed on both donors. The charge switching process in section 3.5 was described by two stable situations, where the electron is located on one of the two donors either $\mathrm{D}_{1}{ }^{+} \mathrm{D}_{2}{ }^{0}$ or $\mathrm{D}_{1}{ }^{0} \mathrm{D}_{2}{ }^{+}$. Therefore one has to consider, if it is possible to create a state in the hydrogen ion (for example by the superposition of the bound and the antibound state), which is mainly located at one of the positive charges. The time evolution of this created state should be in the order of the switching time or even longer.

The addition of a second electron to the two positive charges will make the system comparable to the hydrogen-molecule $\mathrm{H}_{2}$. The energy difference due to the transfer from $\mathrm{H}_{2}$ to $\mathrm{H}_{2}{ }^{+}$would give the ionization energy of the system.

In addition to the treatment of the two donors similar to a molecule, the impact of the nearby (110) surface would have to be included, as well as the influence of the charged tip on the potential landscape. Thus, one should have to solve the Schrödinger equation for the double donor system including the surface, as well as the charged tip. The release of one or two electrons should have to be calculated as a function of the applied voltage and the position of the tip.

However, up to now not even a single donor near the surface can be described quantitatively. For example theoretical calculations for a donor close to a surface show the contrary trend of the binding energy than the measurements [39]. A theoretical description including the influence of the tip is up to now not available. 


\title{
4. Spatially resolved $\Gamma$-X-valley tunneling in an AlAs barrier
}

\begin{abstract}
Resonant $\Gamma$-X-valley tunneling in a GaAs/AlAs heterostructure is observed in the STM experiment for negative tip-sample voltage. For positive tip-sample voltage direct $X$-band tunneling can be verified, which confirms the model of the resonant $\Gamma$-X-valley tunneling at negative tip-sample voltage. The spatial position of the tip where the resonance occurs is dependent on the cleavage surface. The influence of an applied lateral voltage on the resonance can be seen.
\end{abstract}

\subsection{Motivation}

Resonant tunneling was firstly proposed 1973 by Tsu and Esaki [58] and realized one year later by measuring a negative differential conductance in a double barrier GaAs/GaAlAs heterostructure by Chang, Esaki and Tsu [59]. The study of double barriers with pure AlAs has shown that also the X-band does contribute to resonant tunneling features $[60,61]$. Beresford et al. showed that resonant tunneling through the $\mathrm{X}$-valley is also possible for single AlAs barriers [62]. Also nowadays the investigation of $\Gamma$-X-tunneling in single AlAs barriers is still under investigation, it has been shown theoretically that it could lead to spin dependent transport across the heterostructure [63]. Figure 4.1 shows schematically the process of resonant tunneling through X-valley states in a single AlAs barrier. Figure 4.1(a) visualizes the energy band diagram of the structure without applied lateral voltage. The energy of the $\mathrm{X}$-band (red) in AlAs is smaller than the energy of the X-band in GaAs. By applying a lateral voltage (Figure 4.1(b)) a confined state in the AlAs layer can be formed, which is aligned energetically with the $\Gamma$-band of the GaAs. Resonant $\Gamma$-X-valley tunneling is possible as the lattice periodicity perpendicular to the structure is destroyed and thus the momentum in tunneling direction is not conserved. 

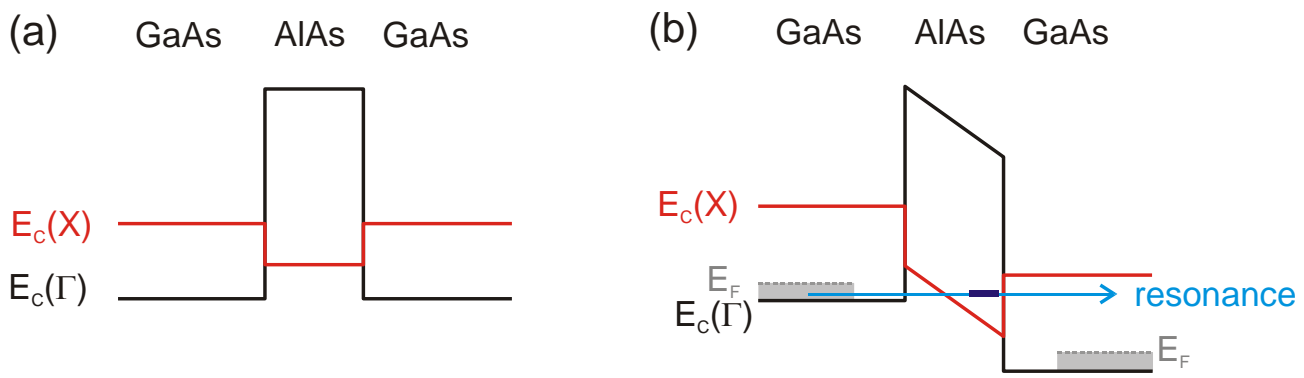

Figure 4.1: Schematic view of the band edge diagram of the single barrier of a GaAs/AlAs heterostructure, (a) without lateral voltage (b) a lateral voltage is applied. The conduction band minimum at the $\Gamma$-point is shown as the black line, the minimum of the X-point as the red line. The X-band profile forms a quantum well and resonant tunneling is possible due to $X-\Gamma$-coupling at the interface through the confined state of the well.

\subsubsection{The GaAs/AlAs heterostructure sample}

Whereas transport measurements of III-V-heterostructures have been intensively studied in the 1970s and 80s, the investigation of heterostructures by cross-sectional STM has not been very intense up to now. In the early years of scanning tunneling microscopy, the investigation of $(\mathrm{Ga}, \mathrm{Al}, \mathrm{In}) \mathrm{As}$ heterostructures started with topography images and single $I(V)$-spectra. Salemink et al. [64], Gwo et al. [65] and Reusch et al. [66] investigated AlGaAs/GaAs interfaces, Zheng et al. [67] investigated InGaAs/GaAs heterostructures, Smith et al. [68] investigated GaAs/AlAs superlattices. The investigation of heterostructures by lateral resolved spectroscopy images has started very recently (2007) by the investigation of pn-junctions by Loth [13] and in 2010 with the investigation of an AlAs/GaAs heterostructure by Wijnheijmer et al. [69]. In the later publication the main focus was on the influence of those structures to manganese clusters, which were embedded close to that structure.

In this chapter an AlAs barrier in GaAs is investigated with the STM. In transport measurements the sample has shown resonant $\Gamma$-X-valley tunneling characteristics [70]. The samples are grown by molecular beam epitaxy (MBE) at the PhysikalischTechnische Bundesanstalt in Braunschweig by K. Pierz in the group of $\mathrm{H}$. W. Schumacher. A detailed description of the growth mechanism can be found in [70] only a brief summary of the structure of the sample system will be given here. The AlAs barrier is $10 \mathrm{~nm}$ thick; the barrier was grown in two steps of each $5 \mathrm{~nm}$ interrupted by $60 \mathrm{~s}$. The interruption was done as the sample is used as a reference sample for a resonant tunneling diode discussed in chapter 5. The structure is embedded in n-doped $\left(2 \cdot 10^{18} \mathrm{~cm}^{-3}\right)$ Si:GaAs. Before and after the AlAs layer the doping concentration in the GaAs is decreased. This undoped region should avoid $\mathrm{Si}$ donors to diffuse into the electric active region. During growth the sample is rotated to obtain a homogeneous surface. The substrate is a silicon doped GaAs (100)-wafer $\left(\mathrm{n}=2 \cdot 10^{18} \mathrm{~cm}^{-3}\right)$. A sketch of the sample layers is presented in Figure 4.2(a) more details of the sample structure can be found in subsection A.3. 

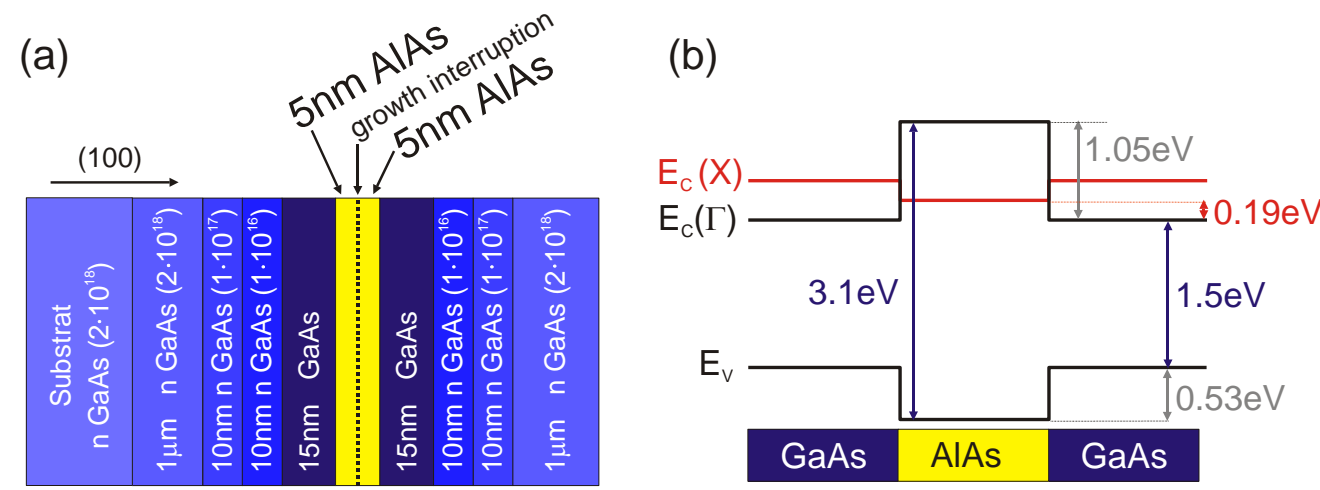

Figure 4.2: (a) The layers of the heterostructure. GaAs is indicated in blue, AlAs in yellow. (b) The expected energy band diagram of the heterostructure according to the literature values for the band offsets. The valence and the conduction band $\left(E_{V}\right.$ and $E_{C}(\Gamma)$ ) are shown in black, the $X$-band minimum $E_{C}(X)$ is shown in red.

According to the literature values of the band gaps and band offsets (introduced in subsection 1.2.1) the energy band diagram which is expected for the heterostructure is presented in Figure 4.2(b). The valence band maximum $\mathrm{E}_{\mathrm{V}}$ and the conduction band minimum of the $\Gamma$-band $\mathrm{E}_{\mathrm{C}}(\Gamma)$ are shown as black lines, the conduction band minimum of the $\mathrm{X}$-band $\mathrm{E}_{\mathrm{C}}(\mathrm{X})$ is shown as a red line. The doping gradient does not have an influence on the energy band diagram; this is discussed in Appendix A.2.

In Figure 4.3 a constant current topography of the heterostructure is shown. The AlAs layer is visible as the dark line which is located vertically in the image between 26$36 \mathrm{~nm}$. In the GaAs area no donor is visible which is due to the low doping in that layer. The atomic corrugation can be seen in the image.

In Figure 4.4(a) a differential conductivity $\mathrm{d} I / \mathrm{d} V(x, V)$-section across the heterostructure is shown. At the top of the image the position of the GaAs and AlAs is marked as blue and yellow boxes. The spectroscopic features of GaAs are discussed in chapter 7 (negative voltage) and Appendix A.2 (positive voltage). The focus of this chapter is on the spectroscopic features of the AlAs layer. At three voltages, a laterally resolved differential conductivity $\mathrm{d} I / \mathrm{d} V(x, y)$-map is shown in Figure 4.4(b-d). For positive voltage the band edge profile between GaAs and AlAs is resolved. One can see that it differs from the expected energy band diagram shown in Figure 4.2(b). This will be described by direct tunneling from the X-band into the tip in section 4.2. At negative voltage features inside the band gap occur. This will be described by resonant tunneling from the $\Gamma$-band of GaAs into the X-band of AlAs in section 4.3. 

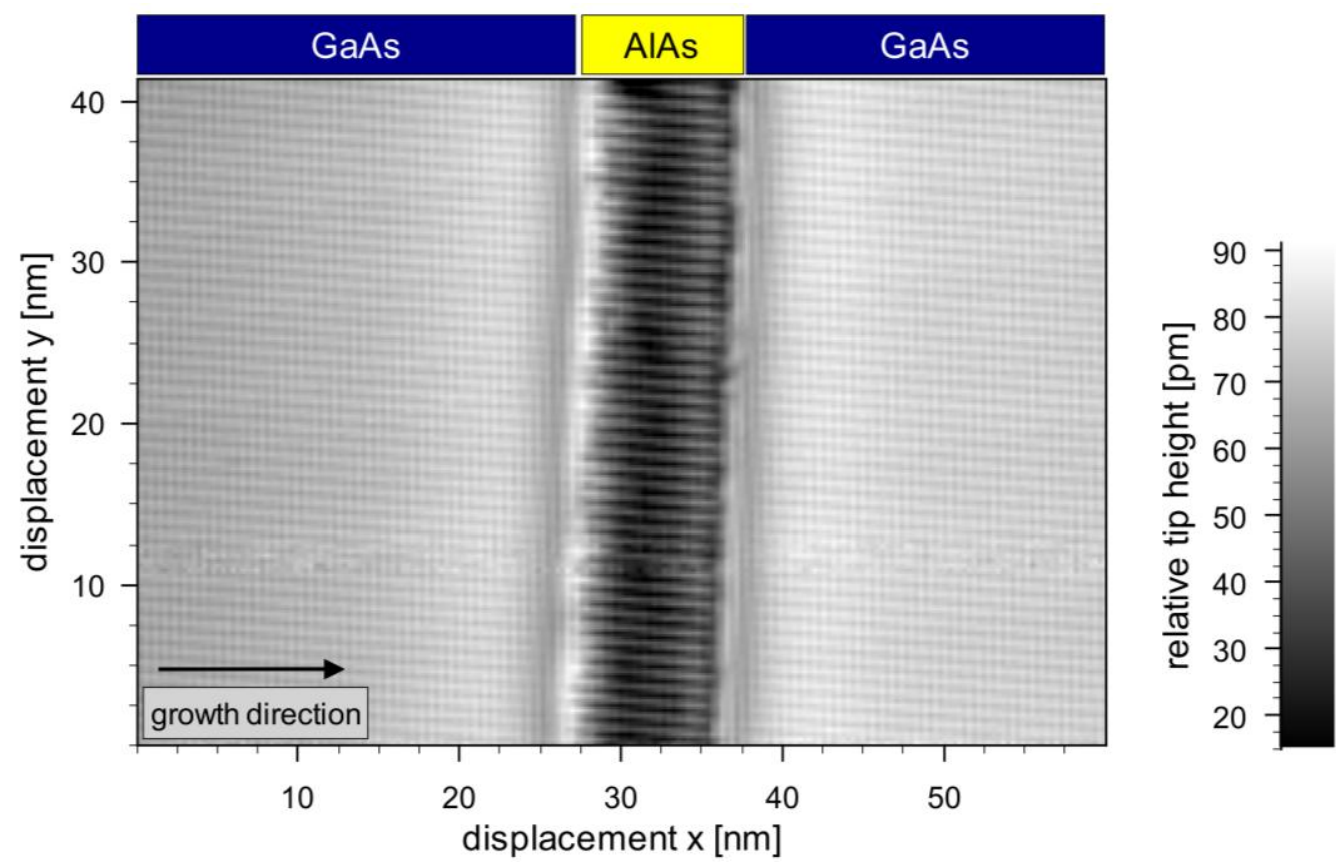

Figure 4.3: Constant current topography of the AIAs layer (marked yellow at the top of the image) embedded in GaAs (marked blue) taken at a setpoint of $-2 \mathrm{~V}$ and $0.1 \mathrm{nA}$.
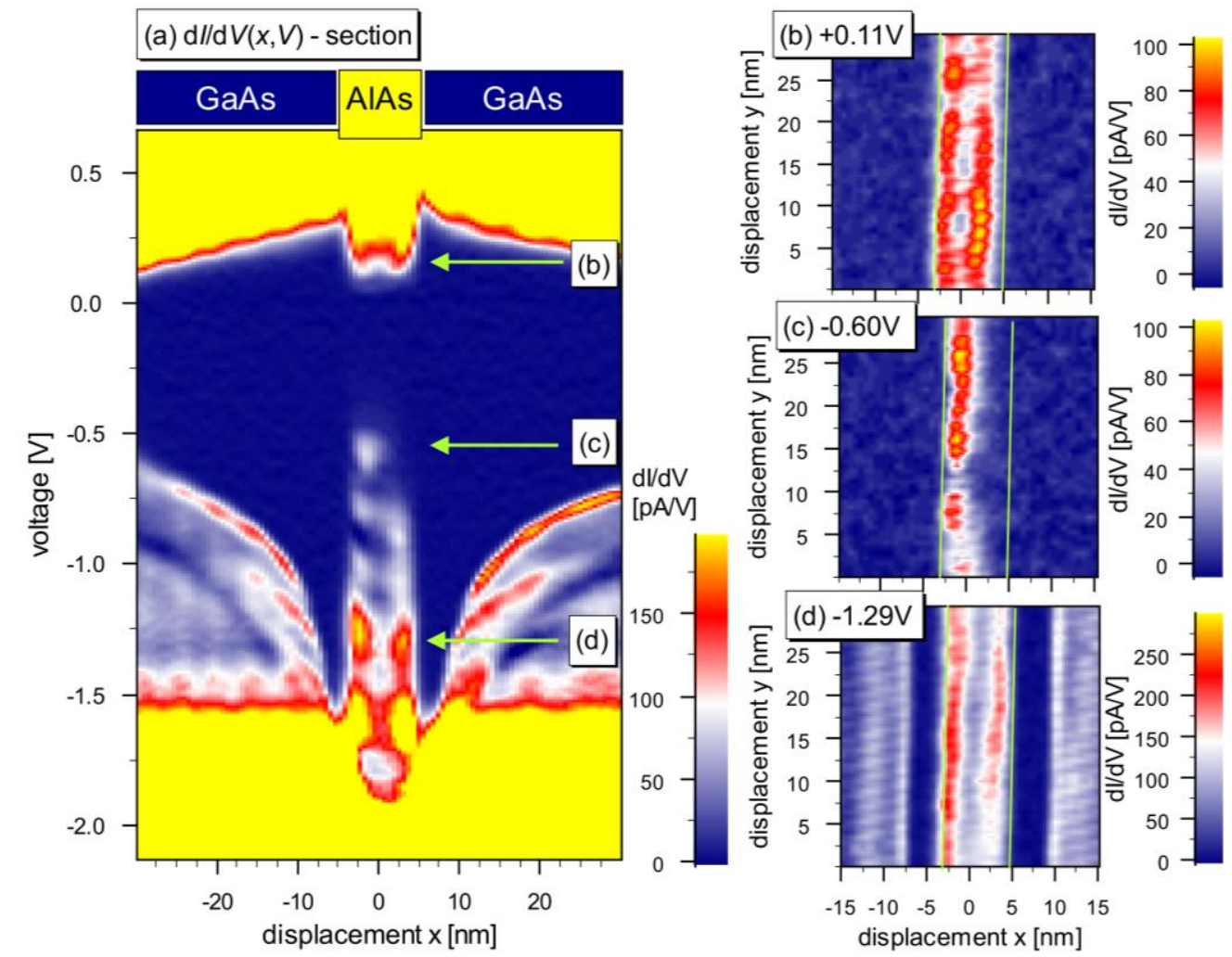

Figure 4.4: A differential conductivity $d / / d V(x, V)$-section across the AIAs barrier is show in (a). The position of AlAs is marked above the image in yellow the position of the GaAs is marked in blue. The spectroscopy in AlAs shows some relevant features marked by the green arrow. At these voltages $d / / d V(x, y)$-maps are shown in (b-c). Here the position of the AIAs is marked by the green line. 


\subsection{Direct tunneling from the X-band of AIAs}

According to the literature values, the conduction band edge offset between GaAs and AlAs at the $\Gamma$-point should be $\Delta \mathrm{E}_{\mathrm{C}}=+1.05 \mathrm{eV}$ (compare Figure $4.2(\mathrm{~b})$ ), thus one would expect $I(V)$-curves on AlAs to be shifted by $+1 \mathrm{~V}$ compared to the $I(V)$-curves on GaAs. Figure 4.5(a) shows a $I(x, V)$-section of the band offset at the interface of GaAs and AlAs. The black lines show contour lines of constant current. At the interface between GaAs and AlAs the contour lines have a positive jump of about $+300 \mathrm{mV}$. This corresponds to the expected positive conduction band offset, but the amount of the jump is a factor of about 3 too small. This is explicable by the following fact: The amount of current at a certain voltage is a measure for the density of states in the corresponding energy range. The density of states is different for the two materials. For AlAs having a larger density of states, the same amount of current would be measured in a smaller energy range than for GaAs. This means that a contour line of constant current is not valid for comparing the band offset quantitatively. Instead one should investigate the conduction band minimum, which is given by the voltage, where the current sets in, which will be called onset voltage.

The topography image of the shown spectroscopy was taken at a voltage of $2.6 \mathrm{~V}$ and 1nA. At this setpoint the tip is relatively far away from the surface (compared to a negative setpoint). The current at small positive voltages is small for large tip-sample distances. It is below the resolution limit of the STM and therefore not detectable.

To enlarge the current at smaller voltage the tip is approached to the sample. To avoid influences due to a change in the topographies, this is not done by simply choosing a
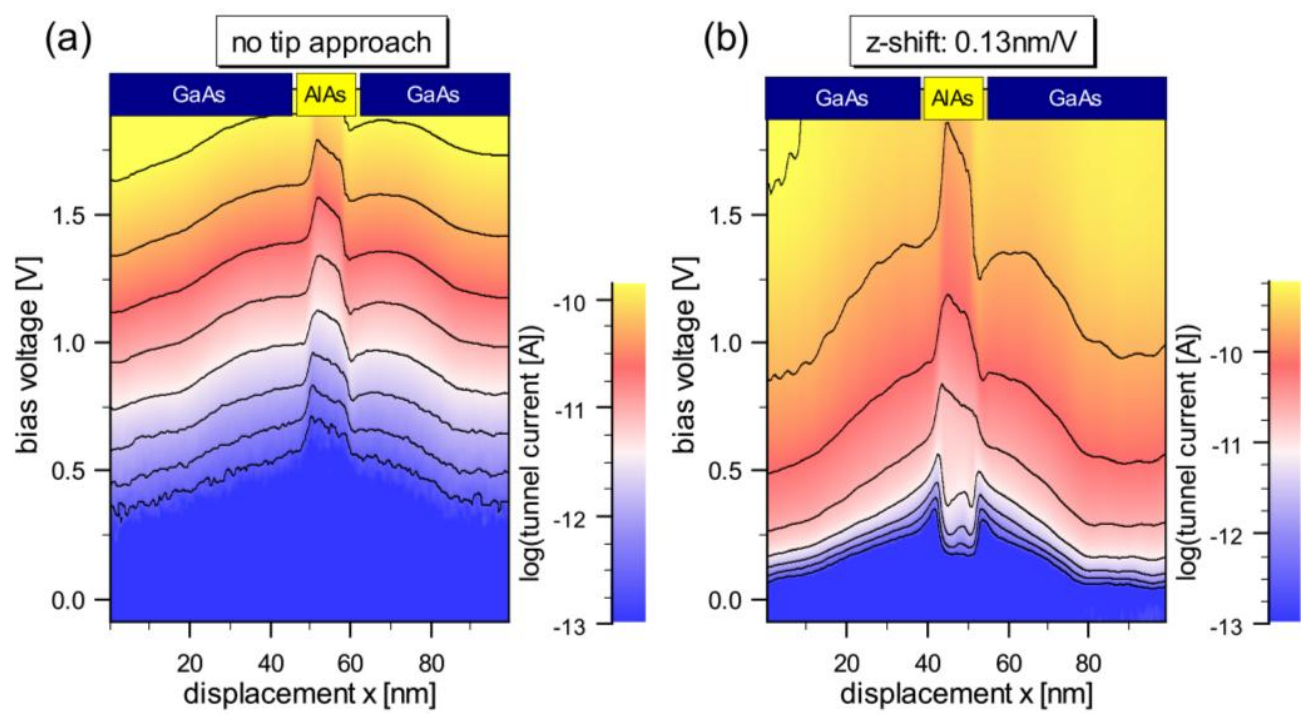

Figure 4.5: In both images $I(x, V)$-sections are shown. The black lines show contour lines of constant tunnel current across the heterostructure. (a) At the intersection between GaAs and AlAs the contour lines jumps to higher voltage, which is expected from the theoretical band offset. (b) To enhance the current in the lower voltage range the tip is approached to the surface during the recording of each $/(V)$-spectrum by $0.13 \mathrm{~nm} / \mathrm{V}$ ( $z$-shift). For high voltage $(>0.6 \mathrm{~V})$ the contour lines show the same trend like in (a), for smaller voltage the contour lines jump to lower voltage at the intersection between $\mathrm{GaAs}$ and AIAs. 


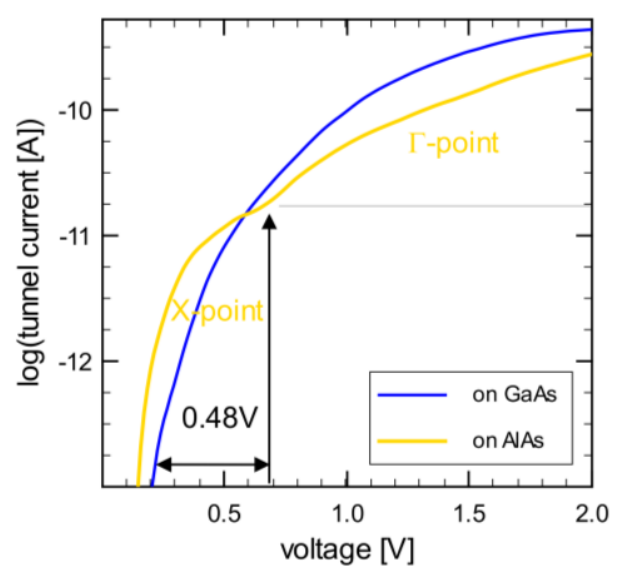

Figure 4.6: I $(V$ ) spectrum taken on GaAs (blue) and on AlAs (yellow). On AlAs an edge is clearly visible (indicated by the black arrow) which is a hint that different states are mapped in the spectroscopy.

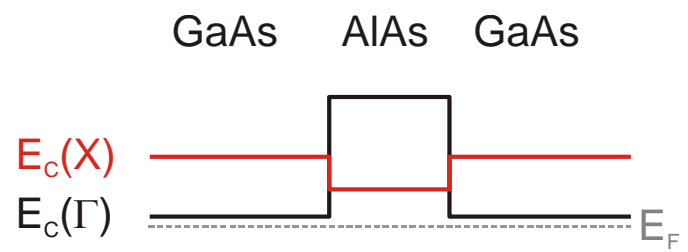

Figure 4.7: Band offset of the heterostructure for the $\Gamma$-band (black) and the X-band (red) according to the literature values.

smaller setpoint voltage. Instead, the voltage setpoint is set to be the same and the tip is automatically approached to the surface at each $I(V)$-spectrum (which is called z-shift). For each single spectrum a z-shift of $1.3 \AA / V$ was applied ${ }^{5}$ (see Figure $4.5(b)$ ). For large positive voltage $>0.7 \mathrm{~V}$ the jump of the contour line of constant current is still positive. Surprisingly, the onset voltage has a negative jump of about $-300 \mathrm{mV}$ at the interface, which is the opposite trend like expected.

In Figure 4.6 a single spectra on AlAs (yellow) is compared to GaAs (blue). At a voltage of about $0.7 \mathrm{~V}$ an edge is visible in the spectrum. Within the effective mass approximation the densities of states of only one band minimum should not have an edge. This leads to the conclusion that the current for voltages smaller than $0.7 \mathrm{~V}$ does not stem from the $\Gamma$-point states but from different states. This is similar to optical measurements [71]. A possible candidate is tunneling from states of the X-point of AlAs. As it was described in subsection 1.2.1, AlAs has an indirect band gap, and the states at the X-point are at a smaller energy than at the $\Gamma$-point. The band edge offset between the $\Gamma$-point of GaAs and the X-point of AlAs should be $\Delta \mathrm{E}_{\mathrm{C}}=+0.19 \mathrm{eV}$. This is smaller than for the $\Gamma$-point but still the onset voltage should have a positive jump. For comparison the band edge alignment is sketched in Figure 4.7. The $\Gamma$-point minimum is shown in black, the X-point minimum in red.

\footnotetext{
${ }^{5}$ This means an enlargement of the current of a factor of about 10 for each volt, which is enough to make the current at small voltages detectable..
} 

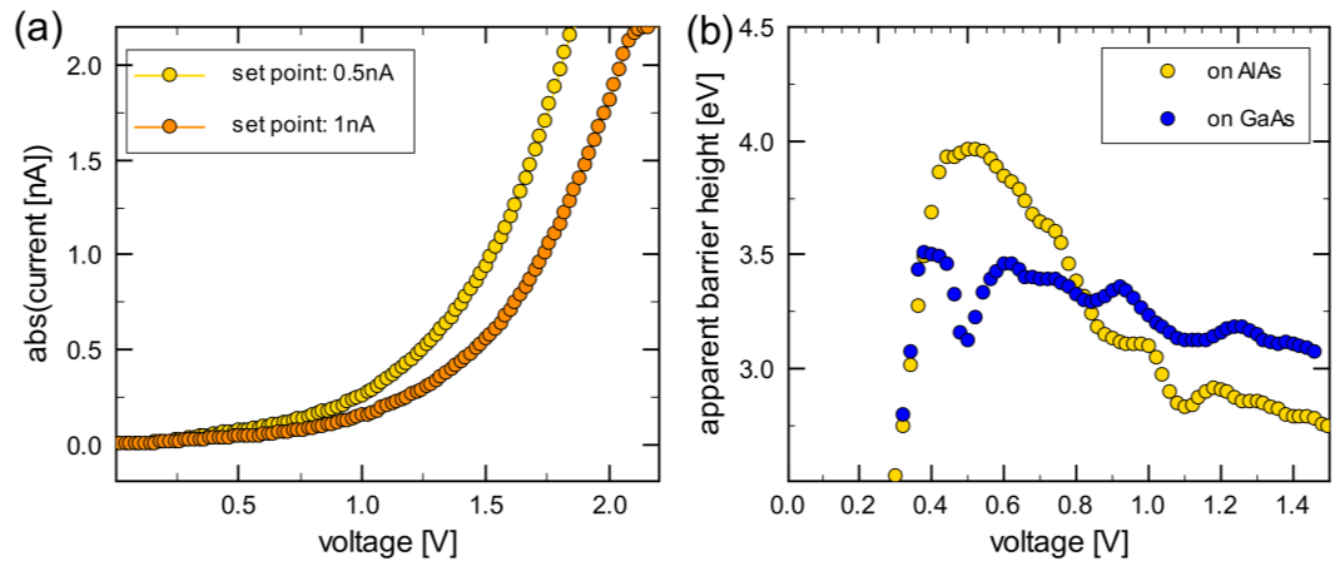

Figure 4.8: (a) As an example two I/ $V$ ) spectra taken at the same lateral position but at two different tip-sample distances from the surface are shown. The squared logarithm of the quotient of the two currents is proportional to the apparent barrier height. Thus, one receives the apparent barrier height as a function of bias voltage, which is shown in (b) for AlAs (yellow) and GaAs (blue)

As a cross check, whether the X-band tunneling could still be a possible explanation a measurement of the voltage dependent apparent barrier height was done. States stemming from the X-band should have a larger apparent barrier height, than states stemming from the $\Gamma$-band. As X-band electrons have a large $\mathrm{k}$ parallel to the surface the measured apparent barrier height is expected to be larger [9]. A change in the apparent barrier height should be visible.

For the voltage dependent apparent barrier height two $I(V)$-spectra are taken at the same lateral position with different tip-sample distance (two different current setpoints were used to stabilize the topography). By using equation (2.1) the two spectra can be compared by building the squared logarithm of the ratio of the current, and a prefactor $K$. The barrier height and the $I(V)$-spectra can be measured simultaneously, (see subsection 2.2.3). This measurement can be used to normalize the extracted apparent barrier height and to eliminate the prefactor $K$.

$$
\phi=K \ln \left(\frac{I_{1}}{I_{2}}\right)^{2}
$$

Figure 4.8(a) shows as an example two spectra taken at a voltage setpoint of $-3.5 \mathrm{~V}$ and a current setpoint of $0.5 \mathrm{nA}$ and $1 \mathrm{nA}$, respectively. Both spectra are taken on AlAs. The voltage dependent apparent barrier height can be extracted from these spectra. It is shown in Figure 4.8(b) for AlAs (yellow dots) and GaAs (blue dots). For AlAs the apparent barrier height is larger in the voltage range of $0.4 \mathrm{~V}$ to $0.8 \mathrm{~V}$ than for higher voltages. This is the range where the $\mathrm{X}$-band tunneling is expected. As a comparison the apparent barrier height of GaAs stays almost constant in the same voltage range. The enhancement of the apparent barrier height is a good proof that X-band tunneling is a possible explanation. 


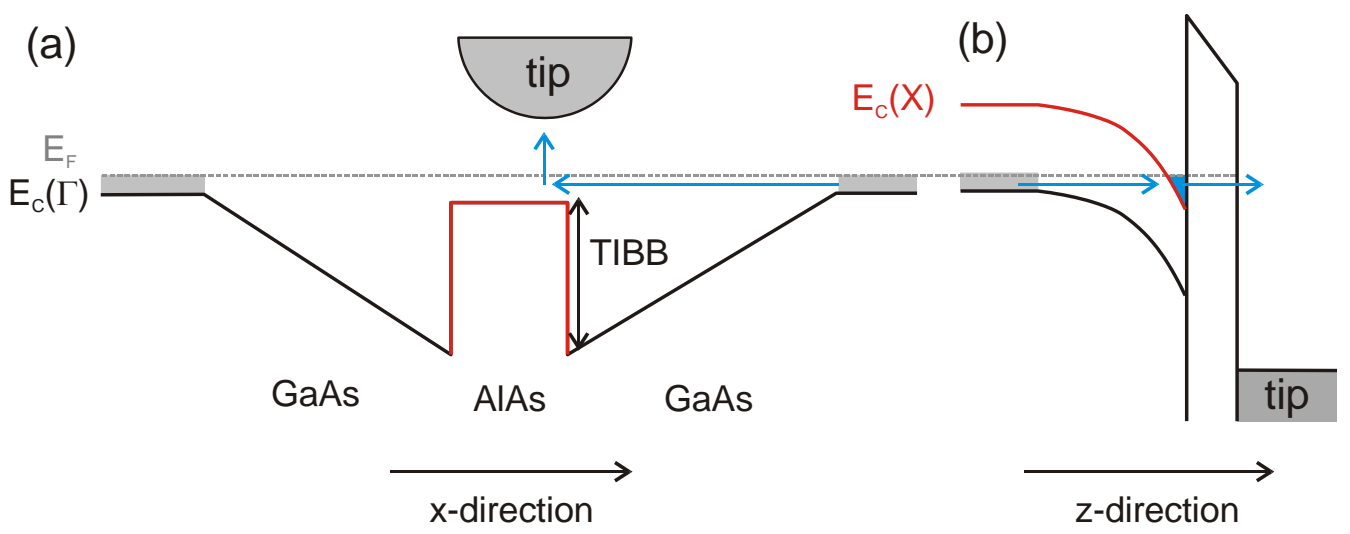

Figure 4.9: Model for resonant $\Gamma$-X-valley tunneling with the help of the tip induced band bending. (a) Energy band diagram parallel to the surface (b) energy band diagram perpendicular to the surface.

\subsection{Resonant $\Gamma$-X-valley tunneling from GaAs to AlAs}

\subsubsection{Model for tip induced resonant $\Gamma$-X-valley tunneling}

In the previous section it has been shown that tunneling from the X-band of AlAs into the tip is possible. In this section a model should be introduced that it is also possible to tunnel resonantly from the $\Gamma$-band of GaAs through a confined state in the $\mathrm{X}$-valley of AlAs into the tip. The X-band in AlAs and the $\Gamma$-band in GaAs have to be aligned. In transport measurements this is realized by applying a lateral voltage across the heterostructure (compare Figure 4.1(b)). In an STM measurement the potential of the tip can be used to align the X-band in AlAs and the $\Gamma$-band in GaAs due to the effect of tip induced band bending (TIBB). This is schematically presented in Figure 4.9, where the energy band diagram is shown for negative voltage with the tip at the position of AlAs. Figure 4.9(a) visualizes the energy band diagram laterally to the surface; Figure 4.9(b) visualizes the energy band diagram perpendicular to the surface. The negative voltage of the tip causes the bands to drop. The X-band of the AlAs well and its confined state is shifted below the Fermi energy of the GaAs contacts. A resonant tunneling from the filled states of the $\Gamma$-band in GaAs through the confined state in the X-valley of AlAs is possible.

A good proof for a resonant tunneling process is the detection of negative differential conductance. According to the model this should be measurable at the position of AlAs. In Figure 4.10 a $\mathrm{d} I / \mathrm{d} V(V)$-spectrum is shown. It was taken at the position of AlAs. The position of the spectrum is marked by the black arrow in the inset which shows a differential conductivity $\mathrm{d} I / \mathrm{d} V(x, V)$-section. At a voltage of $0.7 \mathrm{~V}$ a clear negative differential conductivity is visible and highlighted by the red circle. 


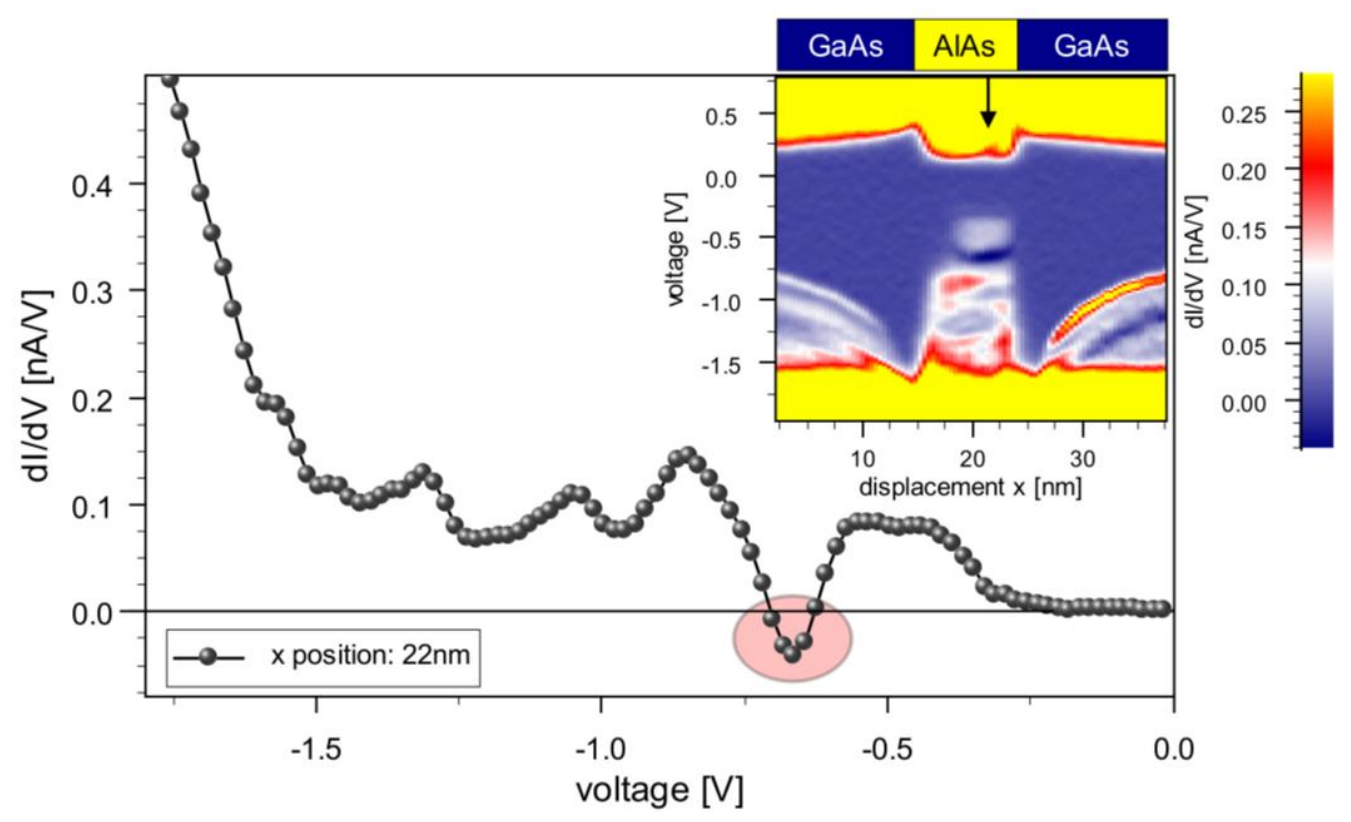

Figure 4.10: Differential conductivity $\mathrm{d} / / \mathrm{d} V(V)$-spectrum taken at the position of AlAs. At a voltage of $-0.7 \mathrm{~V}$ the differential conductance is negative (marked by the red circle). The inset should visualize the tips position while taking this spectrum; the position is marked by the black arrow.

\subsubsection{The influence of the lateral voltage on the $\Gamma$-X-valley tunneling}

In addition to the usual tip-sample voltage a lateral voltage across the heterostructure $\left(V_{\mathrm{Het}}\right)$ can be applied during the STM measurement. This will modulate the band edge profile like in transport measurements, where the $I(V)$-characteristic across the heterostructure is measured. The energy band diagram is schematically shown in Figure 4.11(a) without an applied voltage and Figure 4.11(b) with applied voltage. The amount of TIBB needed to align the confined state of the X-band in AlAs with the Fermi energy of the right contact in GaAs $\left(\mathrm{E}_{\mathrm{F}}{ }^{\text {right }}\right)$ is indicated in the images. If a lateral voltage is applied, a smaller TIBB is needed to shift the confined state of the $\mathrm{X}$-band below the Fermi energy of the right GaAs contact. This means that a smaller tip-sample voltage is needed to measure the resonance. 


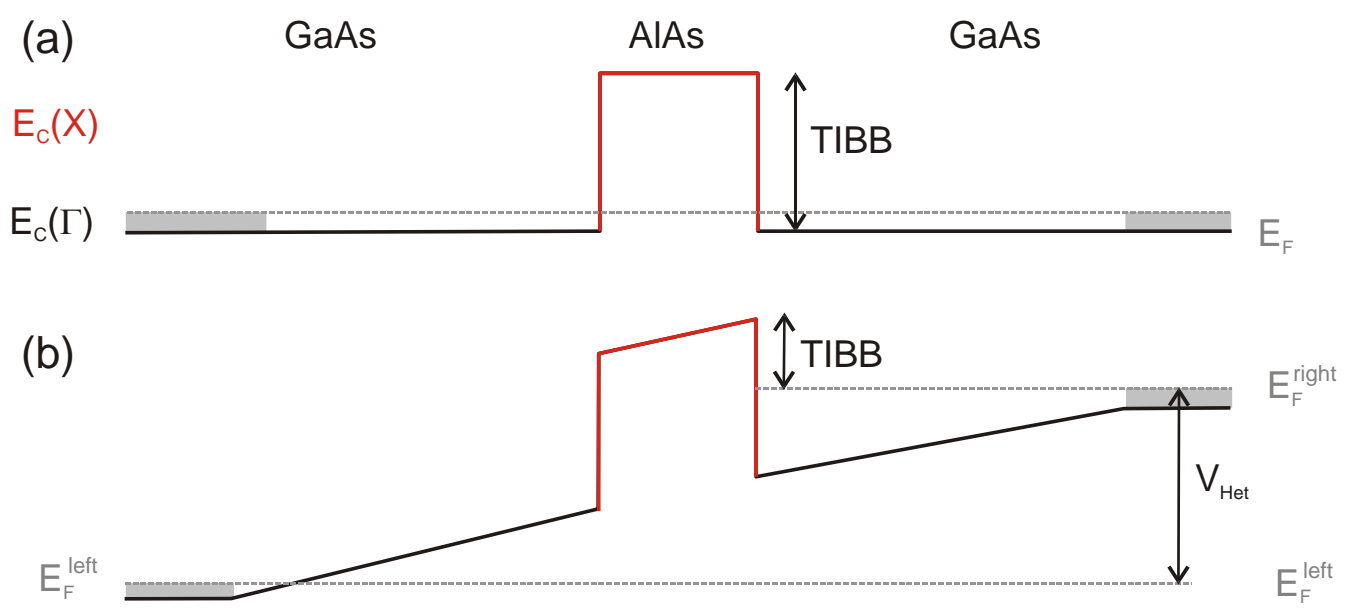

Figure 4.11: (a) Energy band diagram of the GaAs/AlAs heterostructure without applied lateral voltage. Image (b) shows the energy band diagram for the case of an applied lateral voltage across the heterostructure $\left(V_{\text {Het }}\right)$. The amount of TIBB which is needed to shift the confined state in the X-band of AlAs below the Fermi energy of the right contact of the $\Gamma$-band of $\mathrm{GaAs}\left(\mathrm{E}_{\mathrm{F}}^{\text {right }}\right.$ ) is indicated in the images. In (a) the amount of TIBB needed is larger than in (b).

The samples having only a bare AlAs barrier, which were introduced in the beginning of this chapter, were not used for measurement with applied lateral. Instead another set of samples was investigated, which contains InAs quantum dots and which have a smaller AlAs barrier of $2 \times 4 \mathrm{~nm}$ instead of $2 \times 5 \mathrm{~nm}$ (these samples are introduced in subsection 5.1.1.). The features stemming from the AlAs barrier are also visible in theses samples. The differences and similarities of theses samples are compared in Appendix A.3. According to this comparison the samples with the bare AlAs barrier should show the same effect for the applied lateral voltage.

The features in the differential conductivity $\mathrm{d} I / \mathrm{d} V(x, V)$-sections change by applying different lateral voltages $V_{\mathrm{Het}}$. It is presented in Figure 4.12. The sections are taken at the same position at different lateral voltage of (a) $-0.4 \mathrm{~V}$ (b) $0 \mathrm{~V}$ and (c) $+0.2 \mathrm{~V}$. The voltages at the left and right contacts are applied according to equation (2.7). The resonance on AlAs is indicated by the green arrows. For an applied lateral voltage, the resonance is achieved for smaller negative tip-sample-voltage.

For a quantitative analysis of the shift of the resonance, the onset voltage was investigated. The current value corresponding to the onset voltage was chosen to be $-2 \mathrm{pA}$. The onset voltage at the position of the AlAs as a function of lateral voltage is plotted in Figure 4.13. The shift of the resonance is not symmetrically at zero. For an applied lateral voltage of $+0.55 \mathrm{~V}$ the onset voltage is at about $-0.1 \mathrm{~V}$ for an applied lateral voltage of $-0.55 \mathrm{~V}$ the onset voltage is at about $-0.3 \mathrm{~V}$.

In transport measurements the resonance occurs if the confined state of the X-band in AlAs aligns with the Fermi energy of the $\Gamma$-band in GaAs. According to the model for the STM measurement, the tip-sample voltage is used to align the confined state of the $\mathrm{X}$-band in AlAs with the Fermi energy in GaAs. If no tip-sample voltage is needed to obtain the resonance, this should correspond to the case of the transport measurements. Thus, the lateral voltage $V_{\mathrm{Het}}$, at which the onset voltage is expected to 


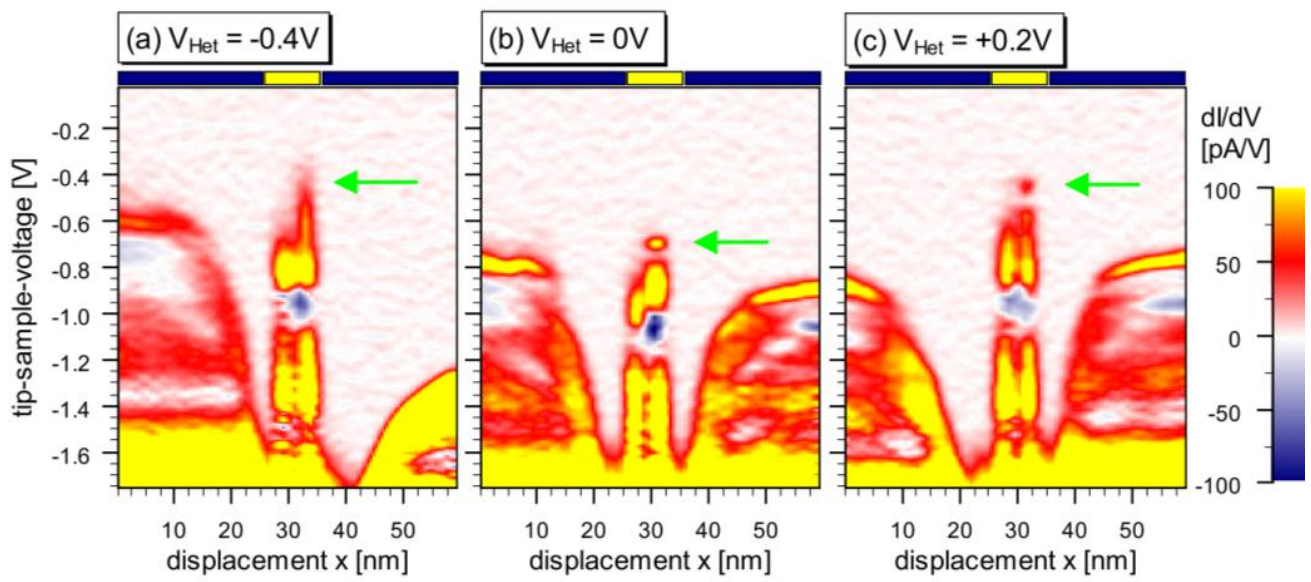

Figure 4.12: differential conductivity $\mathrm{d} / \mathrm{d} V(V, x)$-sections across the heterostructure (AIAs at $30 \mathrm{~nm}$ ) for different lateral voltages $V_{\text {Het }}(\mathrm{a})-0.4 \mathrm{~V}$ (b) $0 \mathrm{~V}$ and (c) $+0.2 \mathrm{~V}$. The first visible peak on the AIAs layer (indicated by the green arrow) shifts for different lateral voltages.

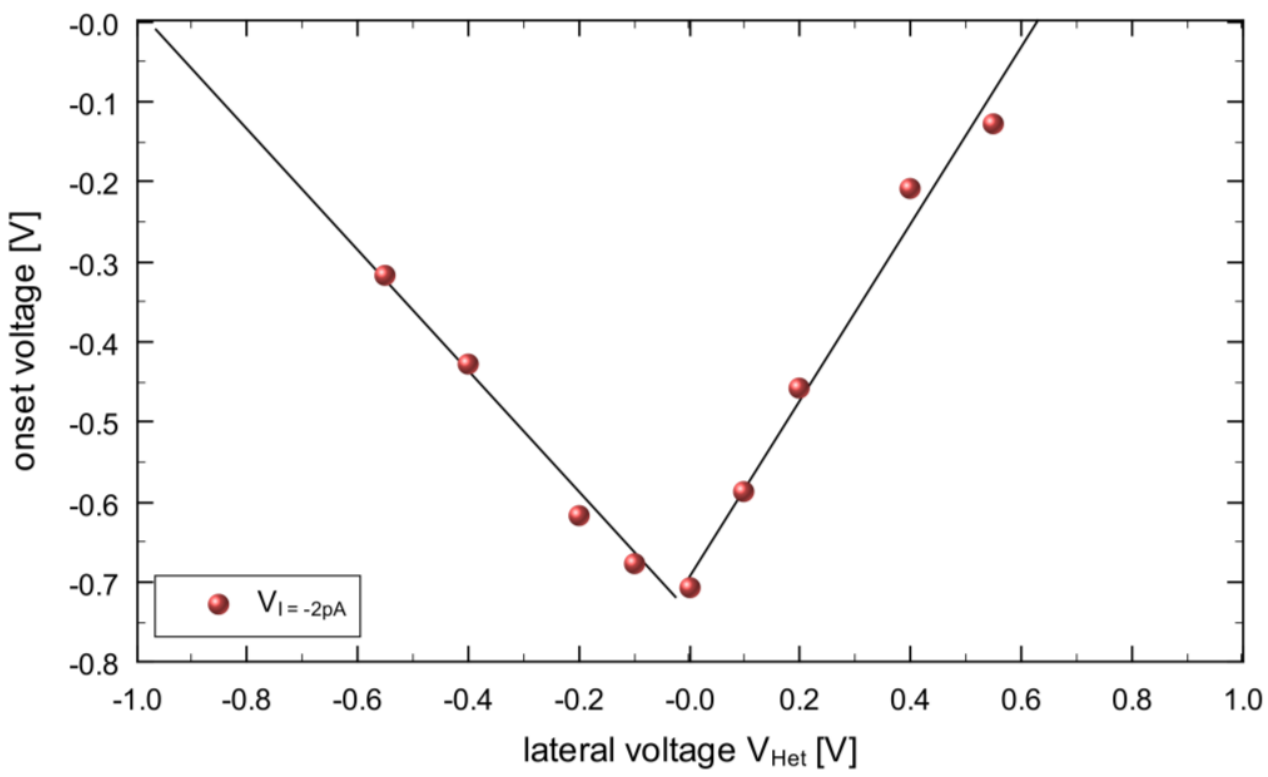

Figure 4.13: Shift of the onset voltage for negative current on the AIAs as a function of the applied lateral voltage $V_{\text {Het }}$. The black lines are a linear fit and are meant as a guide to the eye extending the linear trend of the data points.

be at $0 \mathrm{~V}$, should be comparable to the resonances in a transport measurement. In the case of Figure 4.13 this is expected to be at a voltage of about $V_{\mathrm{Het}}=+0.6 \mathrm{~V}$ and $-1 \mathrm{~V}$ assuming a linear trend.

In Figure 4.14 the results of the transport measurements for comparable samples are presented (Hapke-Wurst, PhD thesis [70]); the sample with bare AlAs introduced in the beginning of this chapter is shown in black. The resonances are marked by the black arrow. Note that those samples have a barrier of $2 \times 5 \mathrm{~nm}$, whereas the samples in Figure 4.13 have a barrier of $2 \times 4 \mathrm{~nm}$. But still one can see that the resonances occur 


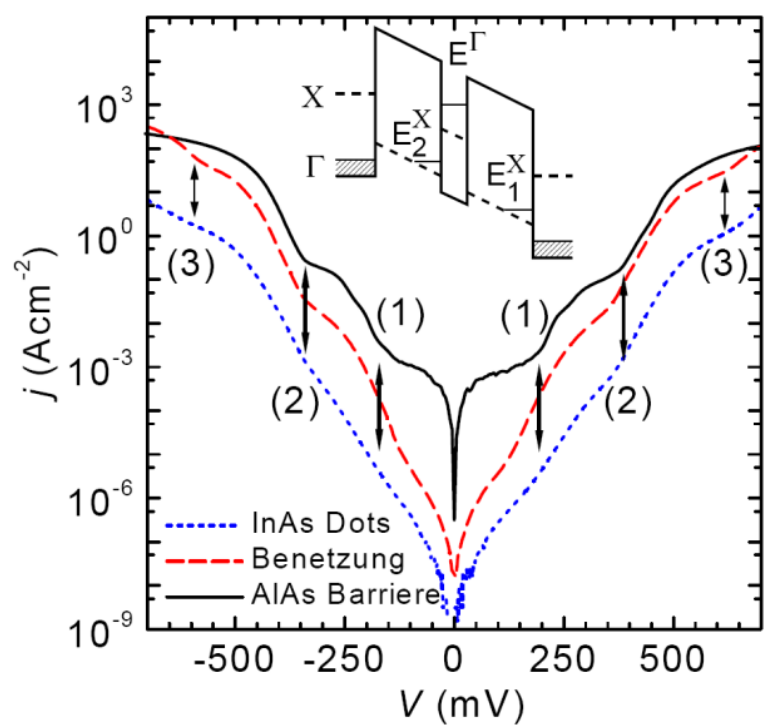

Figure 4.14: Results from the transport measurements from [70]: Current density as a function of applied voltage; the sample with the AlAs barrier is shown in black. The current increase marked with the arrow is stemming from the resonant $\Gamma$-X-valley tunneling.

asymmetrically, for example the first resonance at about $+0.2 \mathrm{~V}$ and $-0.17 \mathrm{mV}$ and the second resonance at about $+0.4 \mathrm{mV}$ and $-0.35 \mathrm{mV}$.

Comparing these values with the STM results, one receives a difference by a factor of about four for the first resonance. The difference could be due to the different AlAs barrier width or surface effects. Also the different measurement technique should not be neglected: An electron in the STM experiment is tunneling from the GaAs to the AlAs into the metal tip. An electron in the transport $I(V)$-characteristic is tunneling from GaAs to the AlAs again to GaAs. As an electron in the metal tip and an electron in the semiconductor $\mathrm{GaAs}$ have different wavelength, the condition for a resonance does not necessarily have to occur at the same energy. This means that the voltage of the resonance is only limited comparable.

\subsubsection{Directional dependence of the $\Gamma$-X-valley tunneling}

In Figure 4.15(a) a differential conductivity $\mathrm{d} I / \mathrm{d} V(x, V)$-section across the heterostructure of the GaAs(0-1-1) surface is shown. The growth direction (100) is indicated by the arrow. The resonance is located on the (100)-side (right side) of the AlAs layer. For a better visualization of this observation Figure 4.15(b) shows a laterally resolved differential conductivity $\mathrm{d} I / \mathrm{d} V(x, y)$-map of that voltage, where the resonance occurs. The position of the AlAs layer is indicated by the green lines.

Figure 4.16 shows the measurement of a sample which was cleaved at the GaAs(01-1) surface. This sample is rotated by $90^{\circ}$ compared to the sample in Figure 4.15 (compare the drawn coordinate systems). The growth direction is also indicated by the arrow. For this surface the resonance is located spatially on the (-100)-side (left side). This observation is presented in the differential conductivity $\mathrm{d} I / \mathrm{d} V(x, y)$-map at the voltage of the resonance in Figure 4.16(b). The position of the AlAs layer is indicated by the green lines. 

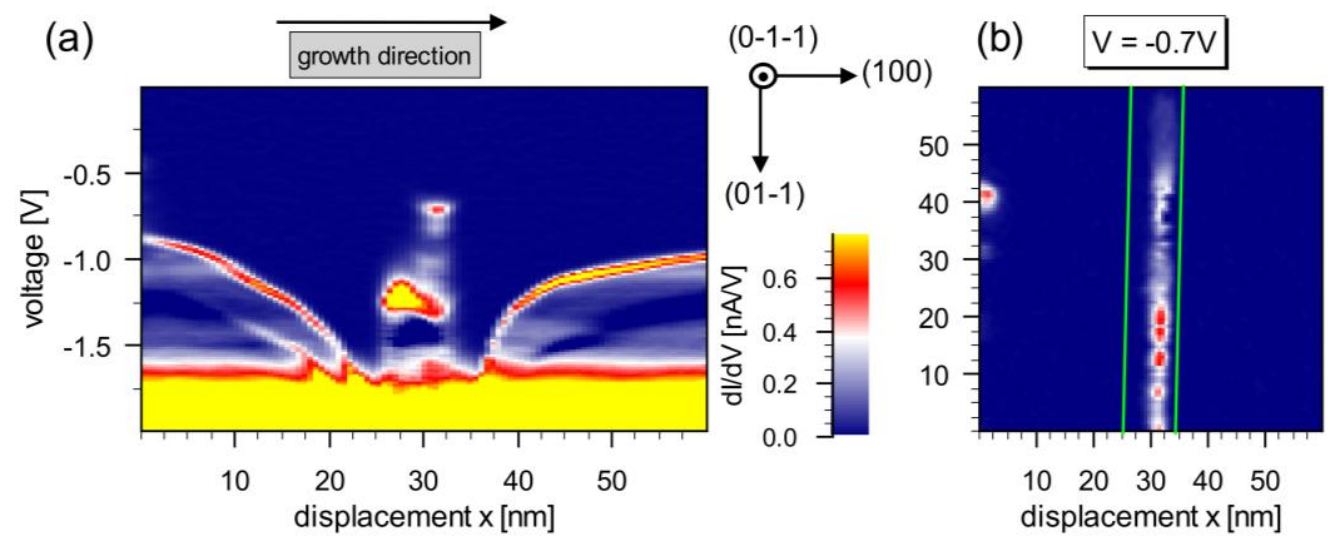

Figure 4.15: (a) differential conductivity $\mathrm{d} / / \mathrm{d} V(x, V)$-section (b) differential conductivity $\mathrm{d} / / \mathrm{d} V(x, y)$-map. The green line indicates the position of the AlAs layer. The cleavage surface is (0-1-1). For negative voltage the peak with lowest energy on the AlAs layer occurs on the right side. (The AlAs layer thickness is $2 \cdot 4 \mathrm{~nm}$ )
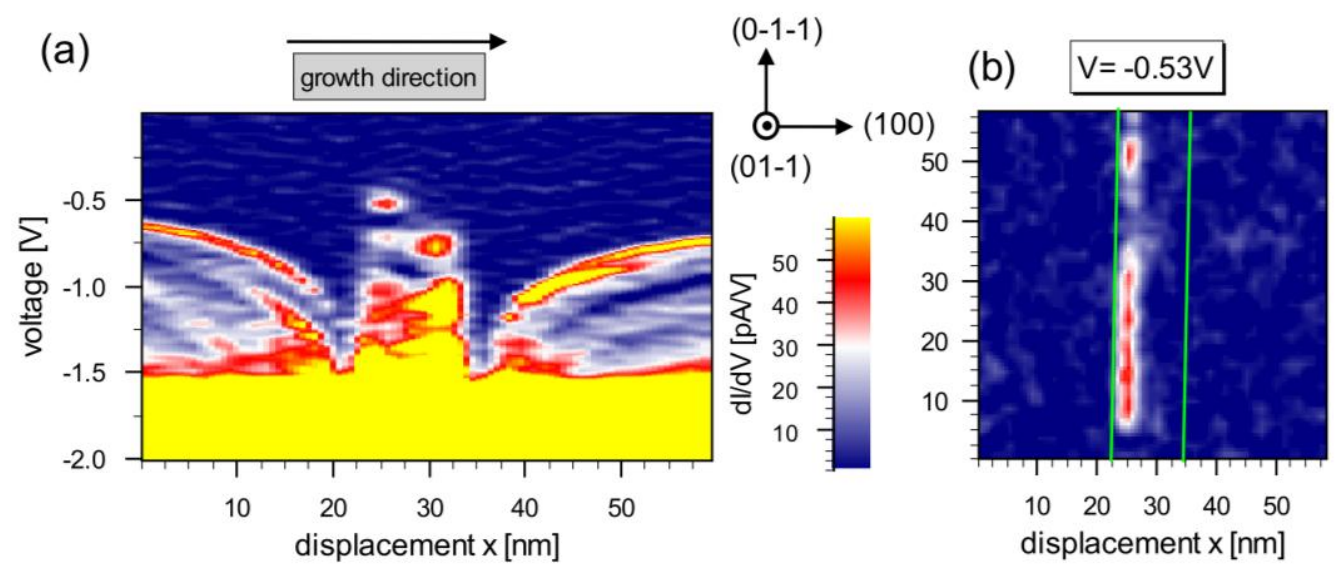

Figure 4.16 (a) differential conductivity $\mathrm{d} / / \mathrm{d} V(x, V)$-section (b) differential conductivity $\mathrm{d} / / \mathrm{d} V(x, y)$-map. The green line indicates the position of the AlAs layer. The cleavage surface is (01-1), which is a rotation by $90^{\circ}$ with respect to the sample in Figure 4.15. For negative voltage the peak with lowest energy on AIAs occurs on the left side, which is opposite with respect to the rotated sample. (The AIAs layer thickness is $2 \cdot 6 \mathrm{~nm})$

The spatial side, where the resonance occurs, seems to be dependent on the direction of the investigated cleavage surface. At this point one should mention that measurements on two different cleavage surfaces for the same sample are not available. However four different samples have been investigated (they are summarized in Appendix A.3). Two of them (P516: $2 \cdot 4 \mathrm{~nm}$ AlAs layer and P519: $2 \cdot 5 \mathrm{~nm}$ AlAs layer) were investigated on the (0-1-1) surface. Both samples show the resonance on the (100)-side (right side). Two samples (P517: 2.6nm AlAs layer and P239: $2 \cdot 5 \mathrm{~nm}$ without InAs) were investigated on the (01-1) and show the resonance on the (-100)-side (left side). The investigation with different tips on each sample did not change the side of the asymmetry, thus effects of an asymmetric tip could be excluded. This is a strong indication that the directional dependence of the cleavage surface is the most promising origin of this effect. 


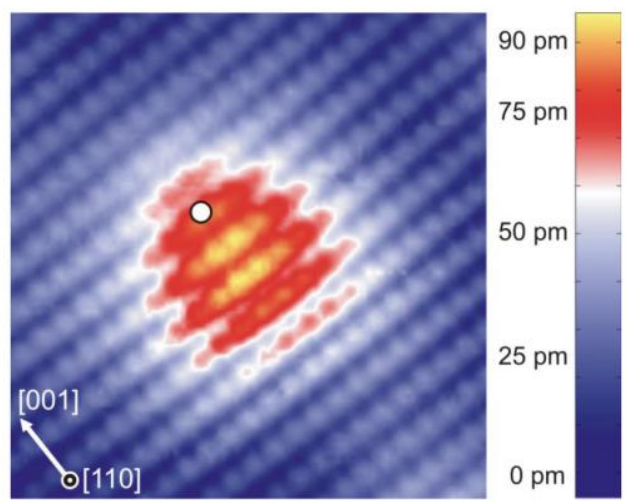

Figure 4.17: STM image (+1.5V and $600 \mathrm{pA})$ of a single carbon acceptor near the (110) cleavage surface of GaAs. The white circle indicates the position of the dopant atom beneath the surface, which can be extracted from topography images at different voltage. Image from [72]

A similar directional dependence of the cleavage surface has also been observed for the acceptor contrast in GaAs, which has an asymmetrically topography contrast in the (001)-direction. This is shown in Figure 4.17, where a topography image of a single carbon acceptor near the (110)-cleavage surface of GaAs is shown. The white circle indicates the position of the acceptor, extracted from a topography image taken at a different voltage. The topographic contrast is located on the (00-1) side. The investigation of a rotated sample with a (-110)-cleavage surface the contrast appears on the (001) side. The origin of this asymmetrical contrast has been explained by Loth et al. [72] by a resonant tunneling channel from the bulk GaAs through the potential of the charged acceptor, into the tip. The two cleavage surfaces GaAs(110) and $\operatorname{GaAs}(-110)$ are not the same. The asymmetry for that transport channel has been explained by enhanced spin orbit coupling for the evanescent states in GaAs. These evanescent states are used for the description of resonant transport [13].

Anyhow further investigation will have to proof, if the direction of the surface is the origin of the asymmetry and if the asymmetry of the resonant $\Gamma$-X-valley tunneling can also be explained by the enhanced spin orbit coupling of evanescence states. 


\section{Quantum dot states inside a resonant tunneling diode}

In this chapter quantum dots states inside a double barrier are investigated. These states are known to be responsible for resonant tunneling phenomena in the diode. The first five wave functions of a quantum dot are compared with the solutions of the quantum mechanical harmonic oscillator problem.

\subsection{Introduction}

In Figure 5.1 a sketch of the resonant tunneling mechanism is presented. In between a double barrier quantum dots are grown. In these quantum dots a quantum mechanical state can exist, which is indicated by the blue wave function in Figure 5.1(a). By applying a voltage $\mathrm{e} V$ this state is energetically aligned with the filled conduction band on the left side and the empty conduction band on the right side (Figure 5.1(b)). Electrons can tunnel resonantly through the barriers. By further enhancement of the applied voltage the state will get off resonance again. One will measure a negative differential conductivity in the $I(V)$-characteristic.
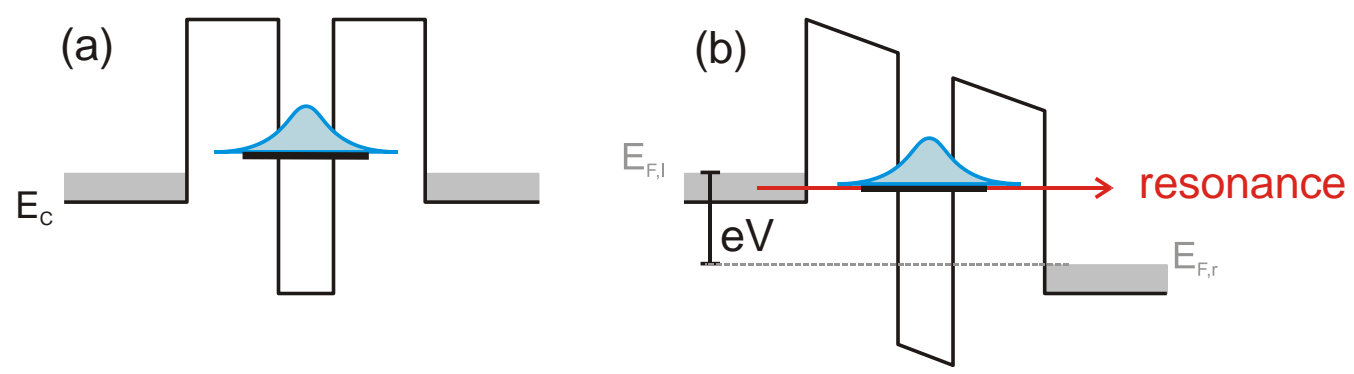

Figure 5.1: Resonant tunneling through quantum dot states: (a) Inside the quantum dot a quantum mechanical state is formed. (b) By applying a voltage this state is aligned with the conduction band of the left contact. Electrons can tunnel resonantly through the barriers. 


\subsubsection{The resonant tunneling heterostructure}

The topographical investigations of InAs quantum dots by scanning tunneling microscopy were recently done by Bruls et al. and Blokland et al. [73, 74]. Offermans et al. showed that strain between the dot and the surrounding matrix causes an outwards bulge of the cleaved surface [75]. The lateral resolved wave function of InAs quantum dots in GaAs were investigated by cross sectional scanning tunneling spectroscopy for cleaved (110) samples in 2000 by Grandidier et al. [76], in 2008 by Uribeta et al. [77] and in 2009 by Girard et al. [78]. Gaan et al. measured the electronic states of InAs quantum dots by single $I(V)$-spectroscopy [79]. The wave function of freestanding InAs quantum dots were investigated on the $\operatorname{GaAs}(001)$ surface by Maltezopoulos et al. in 2003 [80]. The theoretical wave functions of the quantum mechanical particle in a box problem have been compared to measured wave functions of well defined $\mathrm{Pt}$ chains on $\mathrm{NiAl}(110)$ in 2005 by Nilius et al. [81]. Resonant tunneling through quantum dots in a barrier has been studied in 2008 by conductive AFM in an overgrown structure by Tanaka et al. [82].

In this chapter the formation of quantum mechanical states of a resonant tunneling diode is investigated. It is realized by InAs quantum dots embedded in a double barrier consisting of AlAs. The AlAs/InAs/AlAs layers are embedded in GaAs. The cleaved sample system including the STM-tip is schematically sketched in Figure 5.2. The GaAs next to the barrier has the same doping gradient like the sample system described in subsection 4.1.1 and shown in Figure 4.2. The layer system is the same, but additionally after the $60 \mathrm{~s}$ growth interruption, InAs is grown on the sample. The growth interruption is needed to change the substrate temperature. With the chosen growth parameters self organized quantum dots will form for an InAs coverage of 1.6ML. During growth of the quantum dots the sample rotation is stopped, which

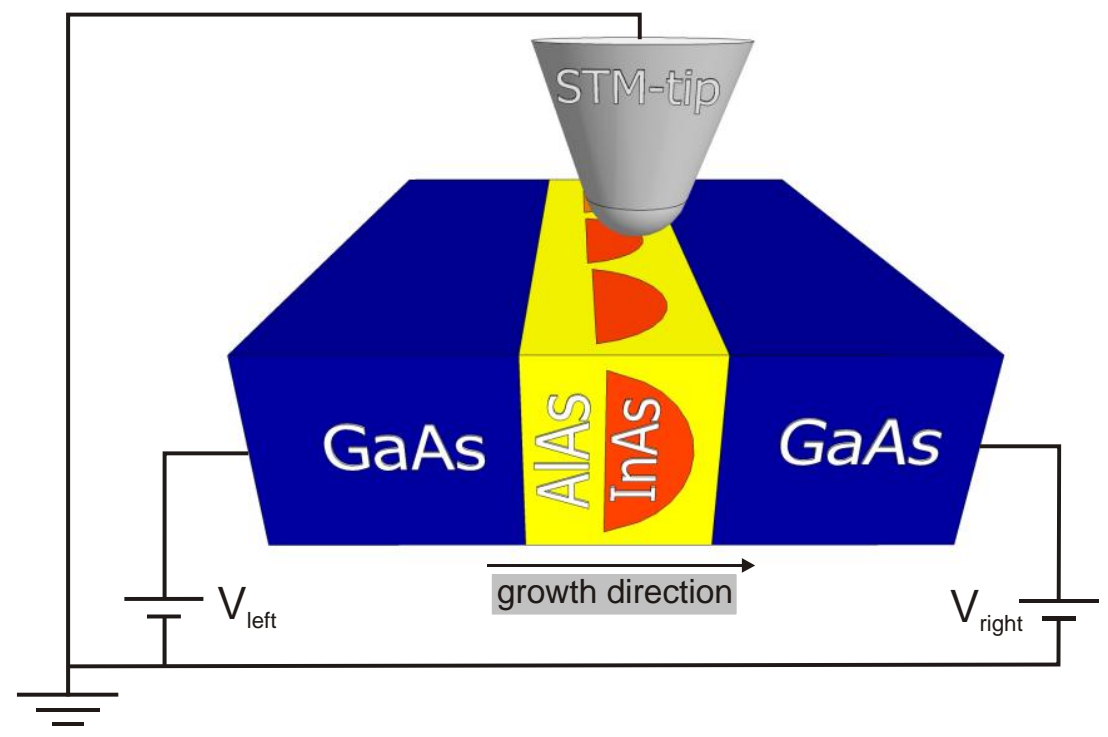

Figure 5.2: Sketch of the sample system. Between two AlAs barriers (yellow) InAs quantum dots are grown (red). The AlAs barriers are embedded inside GaAs (blue). The sample is cleaved perpendicular to the heterostructure, and the cleaved quantum dots can be investigated by the STM-tip. A lateral voltage can be applied; the contacts are labeled $V_{\text {left }}$ and $V_{\text {right }}$, which is according to the growth direction in the shown measured images. 


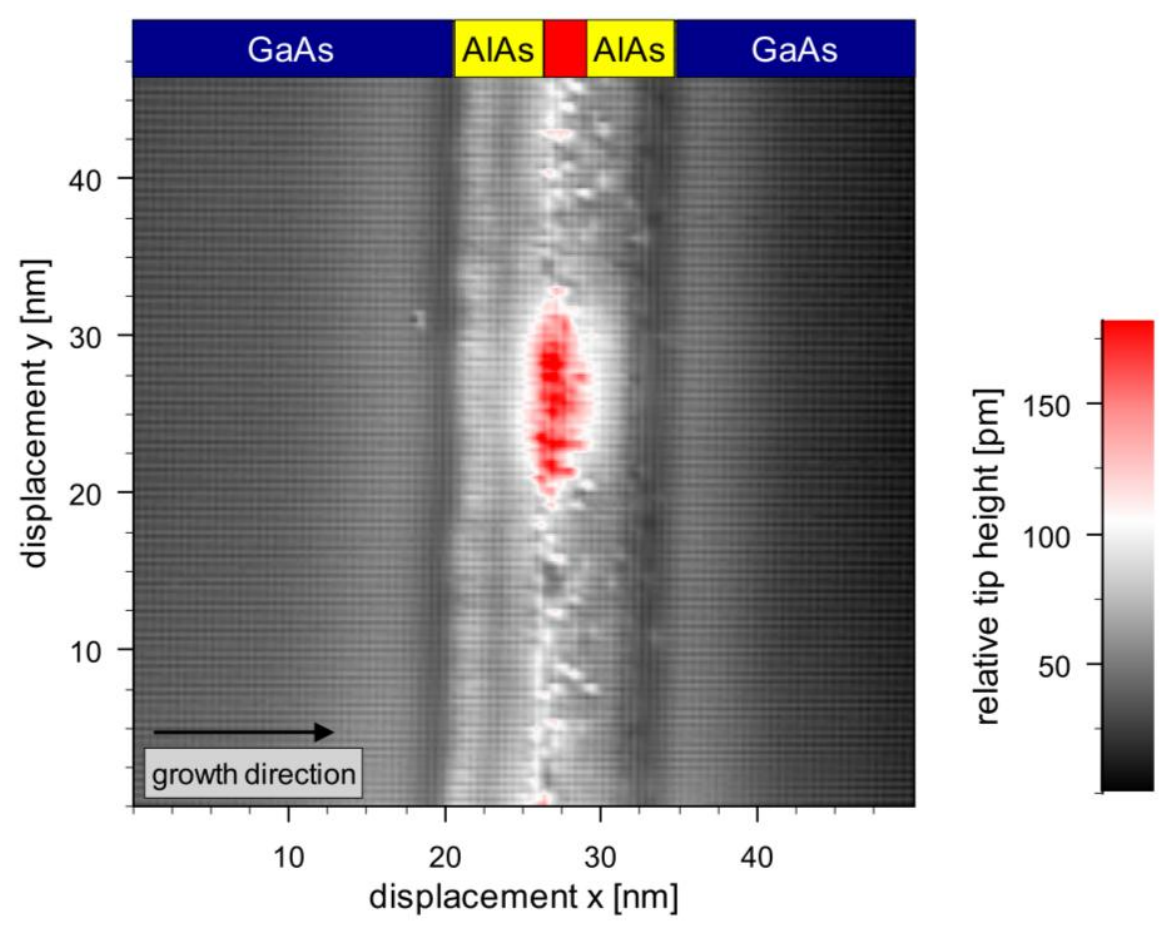

Figure 5.3: Constant current topography image of the AlAs barriers including an InAs quantum dot (setpoint: $-2.4 \mathrm{~V}$ and $0.1 \mathrm{nA}$ ).

leads with the given geometry of sample and effusion cells as well as the growth rate to an InAs covering gradient between $1.55 \mathrm{ML}$ and $2.05 \mathrm{ML}$ in (01-1)-direction of the wafer. This will cause a gradient in the quantum dot size. The sample preparation is described in more detail in [70]. The samples are summarized in chapter A.3.

A constant current topography image of a quantum dot embedded in the AlAs layers is presented in Figure 5.3. The position of GaAs is marked in blue at the top of the image; the AlAs layers are marked in yellow, and the position of the InAs quantum dot is marked in red.

An overview of a spectroscopy is presented in Figure 5.4. The differential conductivity $\mathrm{d} I / \mathrm{d} V(x, V)$-section is shown in Figure 5.4(a). By the green arrow two voltages are indicated. At these voltages the empty and filled quantum dot states are visible. They are shown laterally resolved in Figure 5.4(b) and (c) as differential conductivity $\mathrm{d} I / \mathrm{d} V(x, y)$-maps. The interface GaAs/AlAs is extracted from topography images and is indicated by the green line. The empty states are visible at a voltage of $1.46 \mathrm{~V}$ and the empty states are visible at a voltage of $-1.97 \mathrm{~V}$. The sample has also been investigated by transport measurements, where the $I(V)$-characteristic across the heterostructure sample is recorded. According to those transport measurements, the lowest empty states occur at a lateral voltage of about $0.2 \mathrm{~V}$ for an InAs coverage of $1.8 \mathrm{ML}$ [83]. In the transport measurements this voltage corresponds to the energetic position of the quantum dot with the lowest energy. The macroscopic devices used for the transport measurements contain typically $10^{5}-10^{6}$ quantum dots, which also differs in size. The energy of a quantum dot is dependent on the size of the quantum dot. In the STM measurement only a few quantum dots are investigated, which makes the chance very small to measure one of the quantum dot with the lowest energy. Thus, finding a higher energy in the STM measurements is reasonable. Furthermore, the 

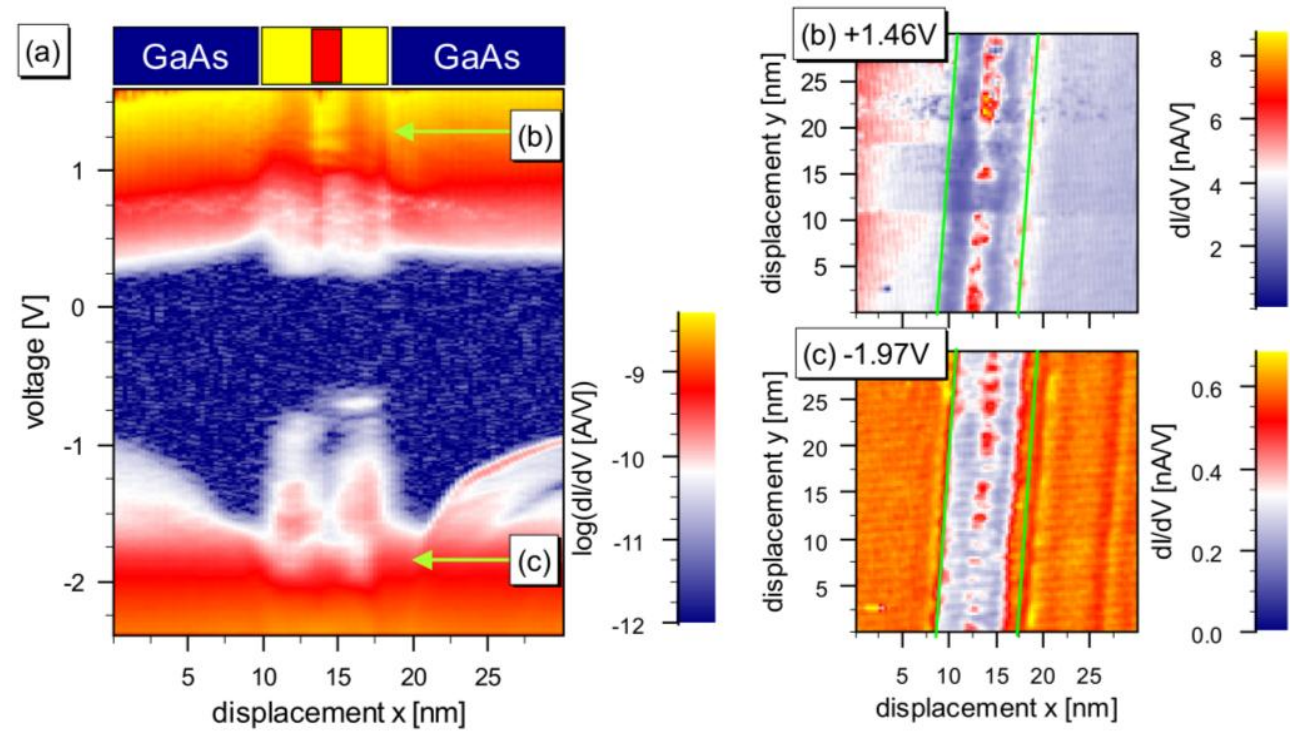

Figure 5.4: (a) differential conductivity $\mathrm{d} / / \mathrm{d} V(x, V)$-section of the resonant tunneling diode. The position of the InAs is marked at the top of the image in red. At positive voltage $(+1.46 \mathrm{~V})$, as well as at negative voltage $(-1.97 \mathrm{~V})$ the quantum dots states can be resolved in the spectroscopy (green arrows). As it is not very clearly visible in the section in (b) and in (c) differential conductivity $\mathrm{d} / / \mathrm{d} V(x, y)$-maps are shown at those voltages. The interface GaAs/AIAs is marked by the green line. The quantum dot states are visible inside the heterostructure.

influence of the tip by the tip induced band bending (TIBB) is important, thus the "real" energy is not directly the applied voltage. The effect of TIBB is large for low doping concentrations, as the screening due to the donor atoms is small. In large scale images it can be seen that the positive onset voltage in the low doped GaAs area next to the AlAs layer is shifted by about $0.5 \mathrm{~V}$ to higher voltages compared to the high doped GaAs area (compare Figure 6.6). As the AlAs layer is also undoped, the energetic position of the AlAs layer as well as the InAs quantum dots will be also shifted by that amount. Another fact is that the sample is cleaved and thus, the InAs quantum dots are close to the surface. Surface effects (like image charge caused by different dielectric constants between the sample and the vacuum or the potential enhancement due to the workfunction) will most likely also change their energy. If the dot is even cleaved itself, its extension is reduced, which enhances the energy of a state. The sample has also been investigated by photo luminescent measurements, the energetic distance between the filled and the empty states has been found to be $1.8 \mathrm{eV}$ [84]. The energetic distance of $3.5 \mathrm{eV}$ measured in the STM (Figure 5.4(b) $+1.46 \mathrm{~V}$ and (c) $-1.97 \mathrm{~V}$ ) is quite good explicable by the above mentioned TIBB effect as well as surface effects. 


\subsection{The quantum dot states compared to the harmonic oscillator}

In this section the wave function of a single quantum dot should be compared to the solutions of the one dimensional quantum mechanical harmonic oscillator. The harmonic oscillator is an important model system for quantum mechanical problems, which has also been used for the description of quantum dots [85]. The most important characteristic of the harmonic oscillator solution of the Schrödinger equation is that the discrete energy eigenstates are equally spaced. The second characteristic is that the number of anti-nodes of the wave function increases by one for each higher energy level, starting with one anti-node for the wave function with the lowest energy. The spatial extension of the wave function increases as well, unlike the wave functions of the particle in a box.

A constant current topography image $\left(27 \times 27 \mathrm{~nm}^{2}\right)$ of an InAs quantum dot is shown in Figure 5.5(a). The green box indicates the area $\left(16 \times 16 \mathrm{~nm}^{2}\right)$ where the spatially resolved differential conductivity is recorded. The laterally resolved differential conductivity $\mathrm{d} I / \mathrm{d} V(x, y)$-maps for different voltages are shown in Figure 5.5(b-f). The applied bias voltage ((b) $0.69 \mathrm{~V}$ (c) $0.80 \mathrm{~V}$ (d) $0.91 \mathrm{~V}$ (e) $1.01 \mathrm{~V}$ (f) $1.10 \mathrm{~V}$ ) at which the

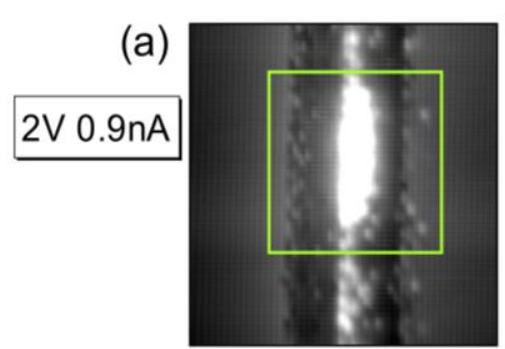

(c)
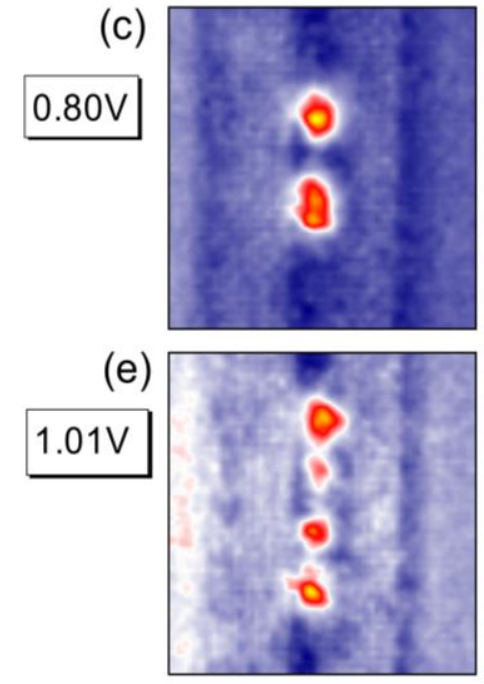
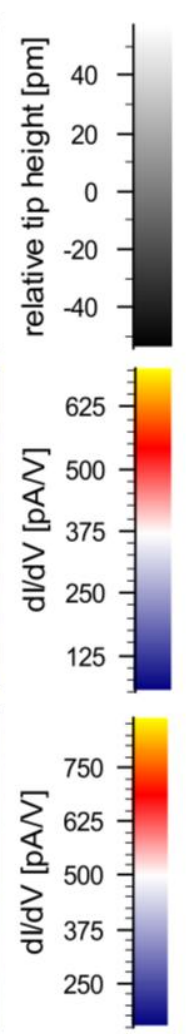

(b)
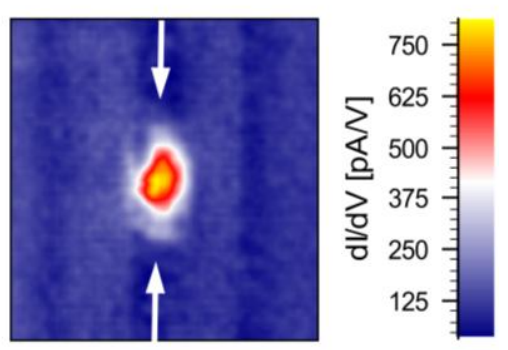

(d)
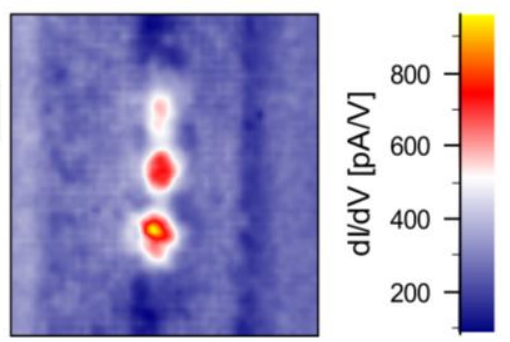

(f)
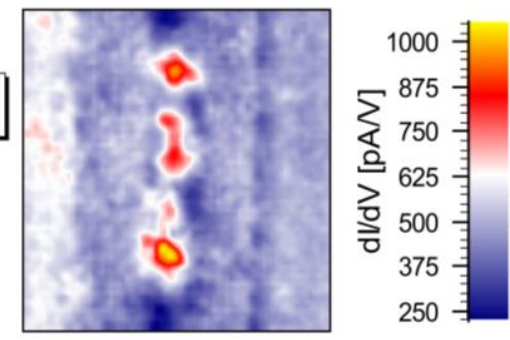

Figure 5.5: A constant current topography image $\left(27 \times 27 \mathrm{~nm}^{2}\right)$ taken at a bias $+2 \mathrm{~V}$ and $0.9 \mathrm{nA}$ of the InAs quantum dot (a). The green box shows the area $\left(16 \times 16 \mathrm{~nm}^{2}\right)$ where the spatially resolved differential conductivity $\mathrm{d} / / \mathrm{d} V(x, y)$-maps in (b-f) are taken. In each image the number of peaks of the wave function is increased by 1 , the voltage is almost equally spaced. 

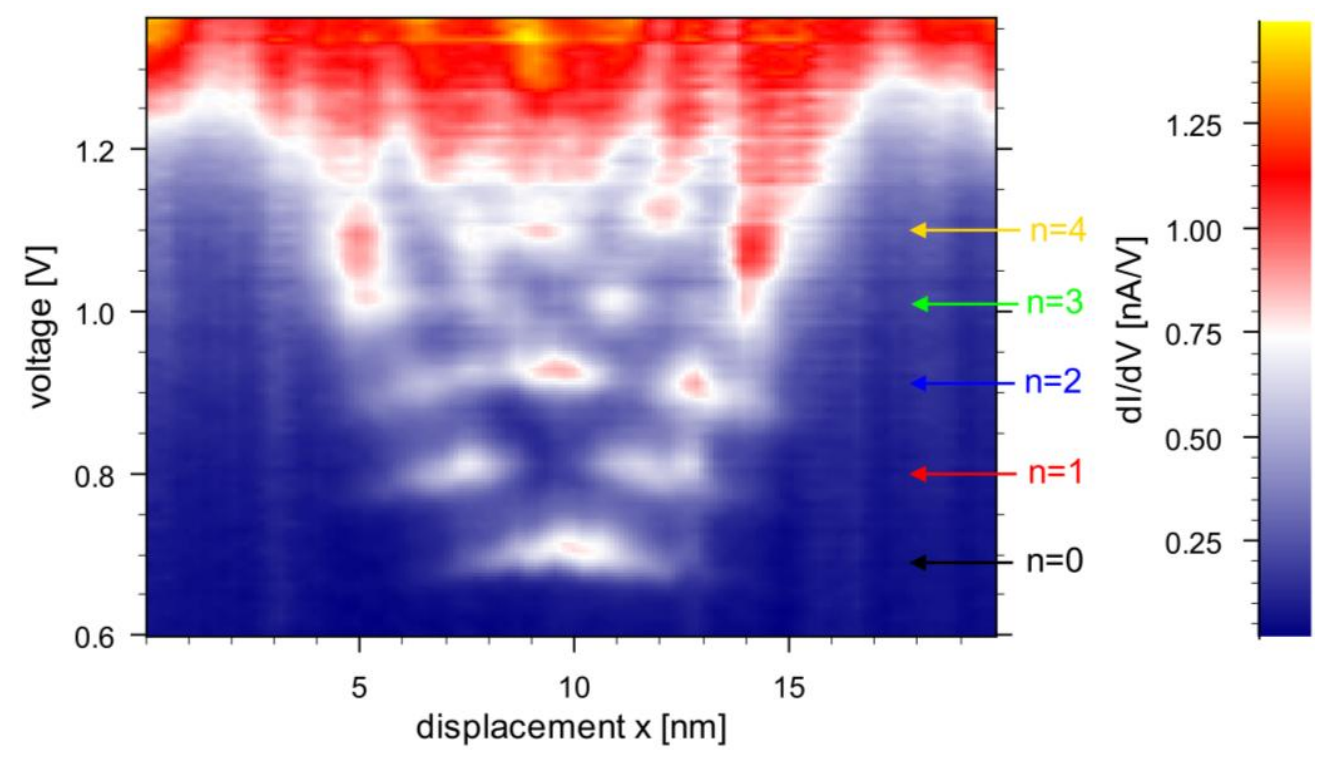

Figure 5.6: $d / / d V(x, V)$-section along the quantum dot. The amount of differential conductivity vanishes between the indicated voltages.

maps are taken are each separated by a voltage of $0.1( \pm 0.01) \mathrm{V}$. From (b) to (f) the number of peaks in the maps increases by 1 . With these two findings the potential of the InAs quantum dot is similar to the above described characteristics of a harmonic oscillator potential.

The similarity becomes even more clear if one visualizes the wave function spatially and energetically resolved, which is done in Figure 5.6, where a $\mathrm{d} I / \mathrm{d} V(x, V)$-section along a line indicated by the arrows in Figure 5.5(b) is shown. The spatial extension of the wave function increases in size, which is expected for the harmonic oscillator potential. Especially for the first three lowest states, one can see that the amount of differential conductivity vanishes between the indicated voltages; that means that different wave functions are mapped. The energetic uncertainty can be estimated from this section to be $\pm 25 \mathrm{mV}$, by using the energetic width of a wave function.

For the comparison with the solution of the Schrödinger equation, one needs the energy difference of the states, as well as the effective mass $m^{*}$ of the electron in the quantum dot. Unfortunately, both values are not exactly known: Due to the influence of the TIBB, the measured voltage difference of $100 \mathrm{meV}$ is not necessarily the exact energy difference of the states. The effective mass for InAs bulk material is known, but the quantum dot with its extension of about $15 \times 15 \times 3 \mathrm{~nm}^{3}$ cannot be treated as bulk like any more. The effective electron mass in this small volume is certainly influenced by the surrounding material.

Luckily the quantum mechanical harmonic oscillator problem can be described by introducing a characteristic length $X_{0}$, where the energy (with $E=\hbar \omega$ ) and the effective mass are both included. Thus, both unknown parameters are reduced to one unknown parameter: 


$$
X_{0}=\sqrt{\frac{\hbar}{\omega m^{*}}}
$$

The solution of the harmonic oscillator potential can be found in every standard quantum mechanics book (for example [50]). With the characteristic length the solution of the wave functions can be calculated and the following equation is found:

$$
\psi_{n}=\left(2^{n} n ! \sqrt{\pi} X_{0}\right)^{-1 / 2} \exp \left(-\frac{1}{2}\left(\frac{x}{X_{0}}\right)^{2}\right) H_{n}\left(\frac{X}{X_{0}}\right)
$$

Here $\mathrm{n}$ is a non-negative integer, labeling the different states and $H_{n}$ are the Hermite polynomials. The corresponding eigenenergies are:

$$
E_{n}=\hbar \omega\left(n+\frac{1}{2}\right) \text { for } n \geq 0
$$

In Figure 5.7 cross sections of the measured differential conductivity $\mathrm{d} I / \mathrm{d} V(x, y)$-maps, taken along a line indicated by the arrows in Figure 5.5(b), are shown (circles). They are compared with the solution of the first five wave functions $(n=0,1,2,3,4)$ of the one dimensional harmonic oscillator potential (solid line). From the measured data a $\mathrm{d} I / \mathrm{d} V$ offset is removed, so that in the area of $-10 \mathrm{~nm}$ to $-8 \mathrm{~nm}$ and $+8 \mathrm{~nm}$ to $+10 \mathrm{~nm}$ the $\mathrm{d} I / \mathrm{d} V$ signal is zero. The $\mathrm{d} I / \mathrm{d} V(x)$ sections are multiplied with a prefactor to coincide the amplitude of the data points with the calculated wave functions. The prefactor is the same for all five data sections. The characteristic length $X_{0}$ is used as the fitting parameter, and is equal for all five wave functions, as well. The fitting is done by minimizing the difference between the data points and the calculated wave functions for all five wave functions simultaneously. The best fit is obtained for a characteristic length of $2.01 \mathrm{~nm}$.

In Figure 5.7 the comparison of the data (circles) and the wave function (solid lines) is shown (both in arbitrary units). The corresponding potential is illustrated by the dashed line. The energy is plotted in the dimension of $\hbar \omega$. The energetic uncertainty of the measurement is indicated by the error bars on the left side of the image.

The measured energetic difference of the states is $100 \mathrm{meV}$. This means that according to equation (5.1) the effective mass is $0.19 m_{0}$. For comparison the effective masses for InAs bulk material are: at the $\Gamma$-point $m^{*}(\Gamma)=0.026 m_{0}$ and at the X-point for the transverse mass $m_{t}{ }^{*}(\mathrm{X})=0.16 m_{0}$ and the longitudinal mass $m_{l}{ }^{*}(\mathrm{X})=1.13 m_{0}[23]$. 


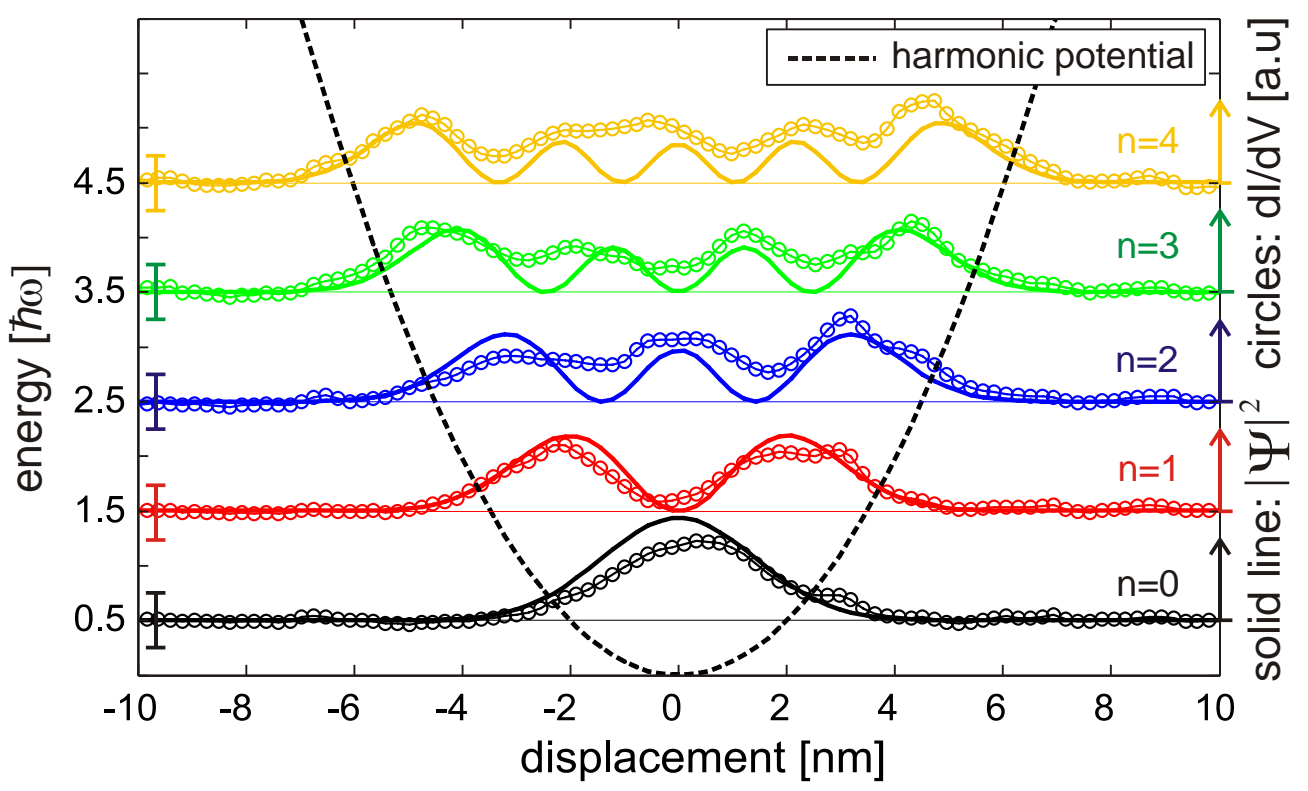

Figure 5.7: The circular data points show $d / / d V(x)$-sections taken along a vertical line through the quantum dot states in the $\mathrm{d} / / \mathrm{d} V(x, y)$-maps in Figure 5.5. The data points are fitted to the eigenfunctions of the harmonic one dimensional oscillator potential (solid lines). The fitting parameter is the characteristic length, the best fit is obtained for $X_{0}=2.01 \mathrm{~nm}$. The potential is drawn in black dotted lines. The energy is plotted in the dimension of $\hbar \omega$; the wave function and the differential conductivity are plotted in arbitrary units.

\subsubsection{Outlook: The influence of a lateral voltage on the quantum dot state}

The main purpose of applying a lateral voltage across the heterostructure was to study the change of the potential landscape in an electric field, which will be described in chapter 6 . In addition to the potential landscape, those measurements can be used to extract the energetic position of the same quantum dot in varying electric fields. It was found that the lateral voltage has an impact on the electric position of a quantum dot state. This subsection is meant as an outlook that further studies of a quantum dot in an electric field could lead to interesting results.

The two contacts of the heterostructure are addressed according to equation (2.8) ( $V_{\text {right }}=V_{\text {STM }}$ and $V_{\text {left }}=V_{\text {STM }}+V_{\text {Het }}$, where left and right is meant to be left and right in the images; compare Figure 5.2). $V_{\text {right }}$ is the side in growth direction (100). The energy of the lowest state of a quantum dot was investigated for 8 different lateral voltages $V_{\text {Het }}$. For each lateral voltage an $I(x, y, V)$-spectroscopy data set was taken. As an example differential conductivity $\mathrm{d} I / \mathrm{d} V(x, y)$-maps taken from three different spectroscopy data sets are shown in Figure 5.8. Each image shows the lowest state of the same quantum dot. The voltage $V_{\text {STM }}$ at which the state occurs is modified by the lateral voltage $V_{\mathrm{Het}}$. 

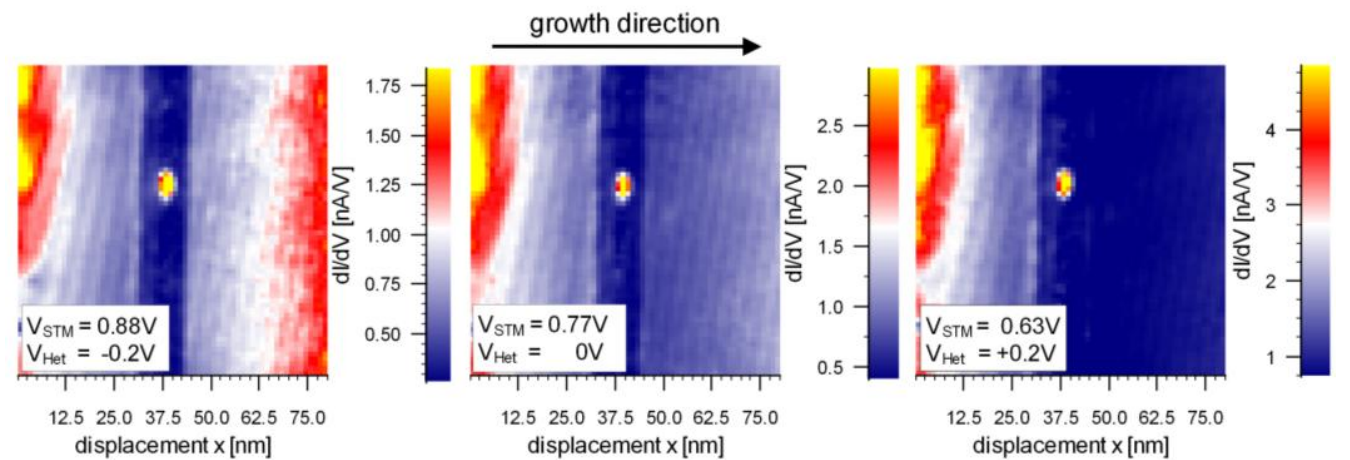

Figure 5.8: differential conductivity $\mathrm{d} l / \mathrm{d} V(x, y)$-maps for different lateral voltages (a) $V_{\text {Het }}=-0.2 \mathrm{~V}$ (b) $V_{\text {Het }}=0 \mathrm{~V}$ (c) $V_{\text {Het }}=+0.2 \mathrm{~V}$. The images are taken at the same position and show the same quantum dot. The voltage $V_{\text {STM }}$ at which the state occours is modified by the lateral voltage $V_{\text {Het }}\left(\right.$ a) $V_{\text {STM }}=0.88 \mathrm{~V}$ (b) $V_{\text {STM }}=0.77 \mathrm{~V}$ (c) $V_{\text {STM }}=0.63 \mathrm{~V}$.

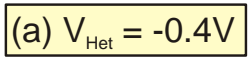

$$
\text { (b) } \mathrm{V}_{\text {Het }}=0 \mathrm{~V}
$$

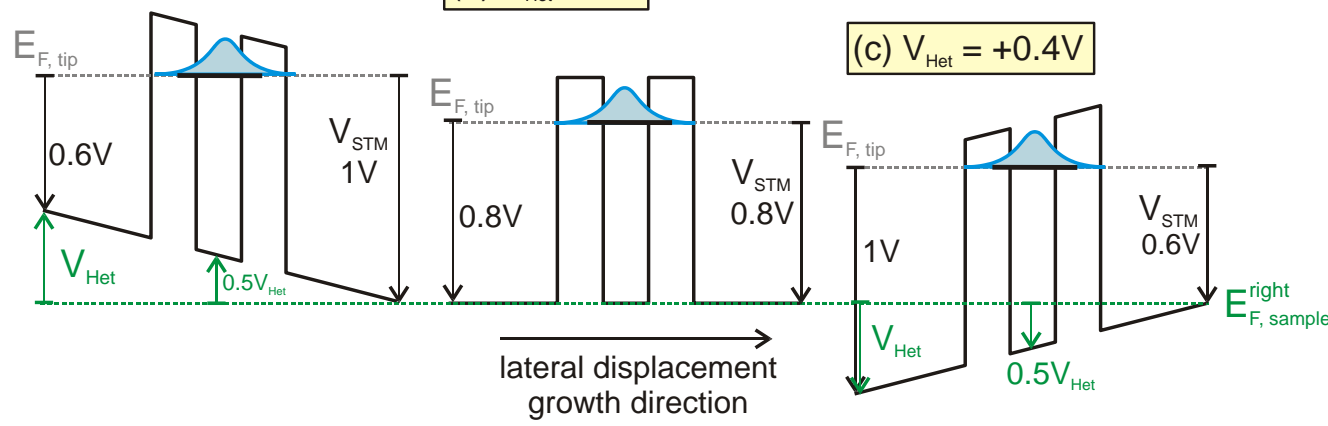

Figure 5.9: Sketch of the quantum dot state for different lateral voltages $V_{\text {Het }}$ (a) $-0.4 \mathrm{~V}$

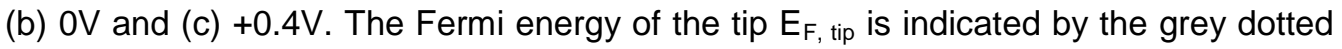
line, the Fermi energy of the right side of the sample is indicated by the green dotted line. The voltage applied on the left and the right side with respect to the tip is labeled.

In Figure 5.9 the energy band diagram of the quantum dot is sketched for three different lateral voltages $V_{\mathrm{Het}}$. The energetic position of the quantum dot state $V_{\mathrm{QD}}$ is counted energetically from the bottom of the well ${ }^{6}$. The Fermi energy of the tip is shown in dotted grey, the Fermi energy of the right side of the sample in dotted green. The applied voltages left and right are labeled. If the voltage drops symmetrically across the heterostructure, the bottom of the well is shifted linearly by $-0.5 \cdot V_{\mathrm{Het}}$, thus the voltage at which the state occurs should be shifted according to $V_{\mathrm{STM}}=-0.5 \cdot V_{\mathrm{Het}}+V_{\mathrm{QD}}$. For a better visualization this shift $\left(-0.5 \cdot V_{\mathrm{Het}}\right)$, which is only a measurement artifact, is subtracted from the data, shown in Figure 5.10. In this figure the energetic position of the quantum dot state $V_{\mathrm{QD}}$ is shown as a function of the lateral voltage $V_{\mathrm{Het}}$. By an error evaluation (similar to the one, which will be described in subsection 6.1.1) the resulting error from the measurement can be determined to $\sim 8 \%$ of the applied lateral voltage, it is plotted to the data. It seems that the lateral voltage

\footnotetext{
${ }^{6}$ Note that the material of the quantum dot is InAs, so the bottom of the well should lie energetically deeper than the conduction band of the GaAs. This is not included into the sketch.
} 


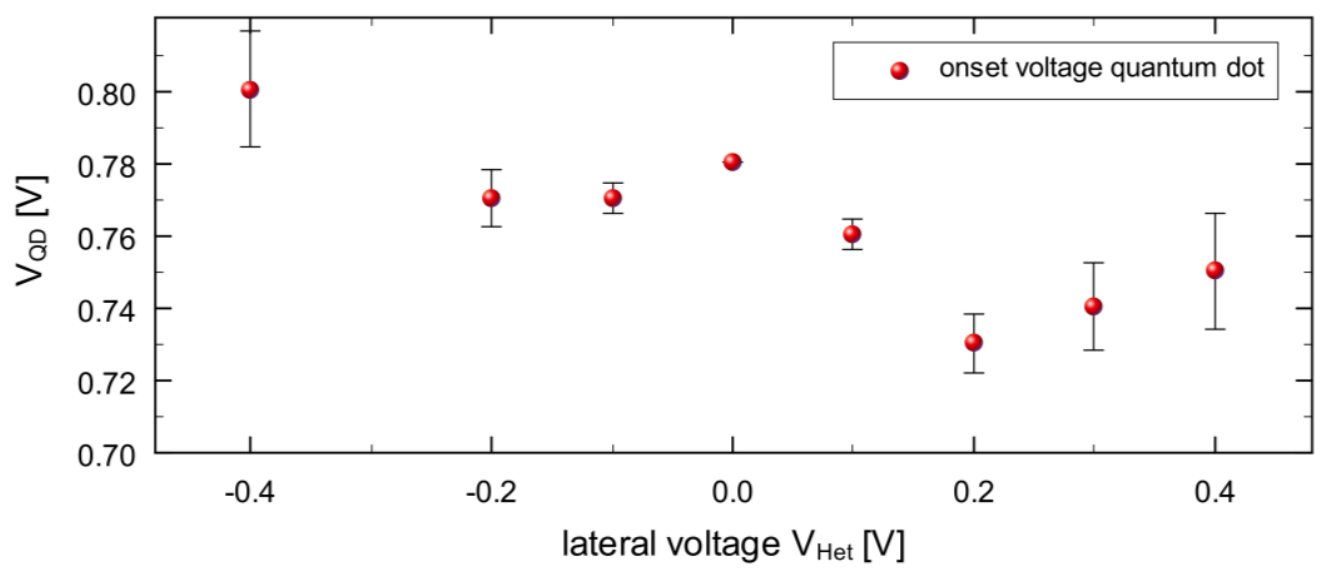

Figure 5.10: The energetic position of the quantum dot state $V_{\mathrm{QD}}$ as a function of the lateral voltage $V_{\text {Het }}$. An impact of the lateral voltage is clearly visible.

has an influence on the energy of the quantum dot state, which could be probably due to the Stark shift. The influence of the lateral voltage on these quantum dot states are a promising candidate for further investigation. 


\section{Measuring the potential landscape of a biased resonant tunneling diode}

The potential landscape in heterostructures is a crucial parameter for the interpretation of transport measurements. In this chapter it is shown that the extraction of the potential landscape in a biased resonant tunneling diode is possible with the STM. The use of laser radiation gives direct access to the intrinsic band edge profile.

\subsection{Extracting the potential landscape inside a biased resonant tunneling diode}

The potential of a biased semiconductor heterostructure was measured in 1987 by Muralt et al. [86] by scanning tunneling potentiometry. The potential of a pn-junction was measured by Jäger $e t$ al. [87]. The potential of a Schottky contact was extracted with scanning tunneling microscopy by Reusch et al. [88] [89] and Winking [90]. Scanning tunneling microscopy on biased devices was investigated by Cobley et al. in 2007 [91]. The potential of a biased pin-diode was measured by scanning tunneling spectroscopy by Loth [13] 


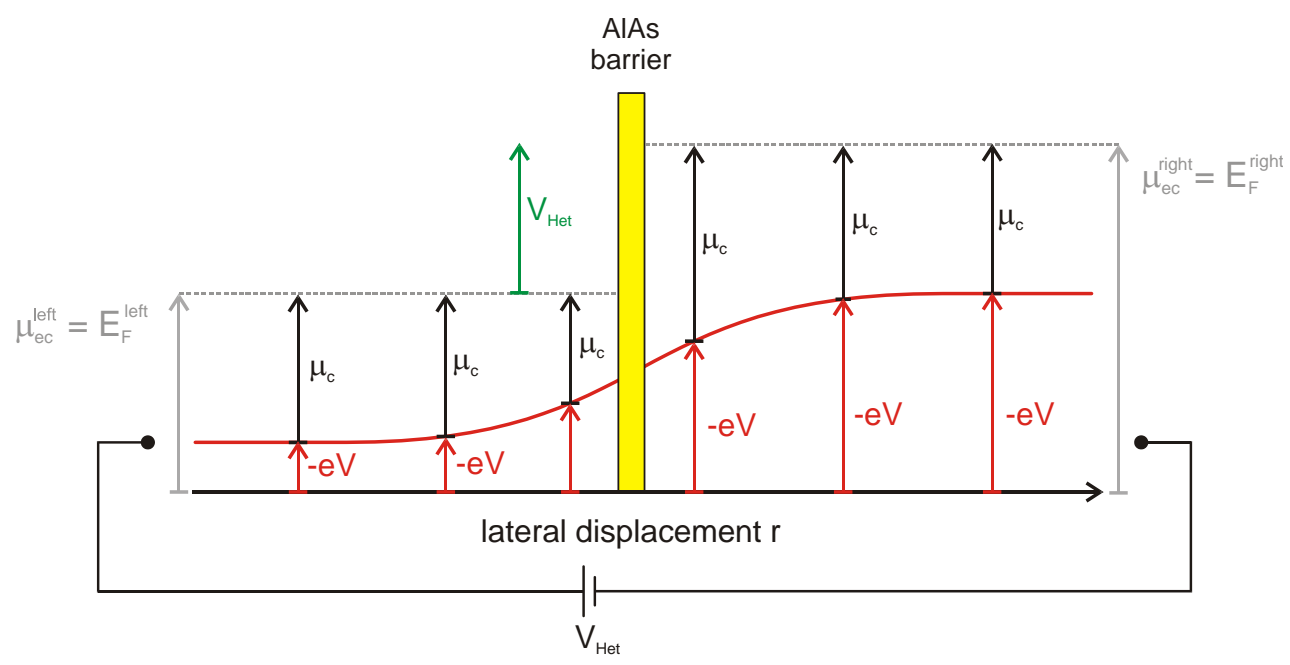

Figure 6.1: Band edge profile of the heterostructure with an applied lateral voltage $V_{\text {Het. }}$ The GaAs sample and the AlAs barrier (yellow) is shown laterally resolved. The electrochemical potential (also referred to as Fermi energy $\mathrm{E}_{\mathrm{F}}$ ) of the left contact $\mu_{\mathrm{ec}}^{\text {left }}$ is shifted energetically with respect to the electrochemical potential of the right contact $\mu_{\mathrm{ec}}{ }^{\text {right }}$. The resulting electrostatic potential is shown as the red line -e $V(r)$. As a result charges are reorganized and the chemical potential $\mu_{c}(r)$ changes locally.

The applied lateral voltage $V_{\text {Het }}$ will affect the potential landscape of the heterostructure. It can be solved by the Poisson equation. The energy band diagram is schematically sketched in Figure 6.1. The electrochemical potential of the right and left contact $\mu_{\mathrm{ec}}^{\text {left }}$ and $\mu_{\mathrm{ec}}^{\text {right }}$, are energetically shifted to each other by the applied lateral voltage $V_{\text {Het: }}$ :

$$
\mu_{e c}^{l e f t}=\mu_{e c}^{r i g h t}+V_{H e t}
$$

The AlAs barrier of the sample works for the electrons as a tunnel barrier. The resistivity of that barrier is large compared to the resistivity of homogeneous GaAs. (It will be estimated in the end of this section). Thus, the electrochemical potential is constant on each side. The electrochemical potential $\mu_{\mathrm{ec}}$ is the sum of the chemical potential $\mu_{\mathrm{c}}$ and the electrostatic potential $V$. The two latter quantities can vary in space $r$ :

$$
\mu_{e c}=\mu_{c}(r)-e V(r)
$$

The electrostatic potential $V$ is multiplied by the electron charge -e. In semiconductor physics the electrochemical potential $\mu_{\mathrm{ec}}$ is usually called the Fermi energy. Thus, in the images of this thesis it is labeled by $\mathrm{E}_{\mathrm{F}}$.

The electrostatic potential $V(r)$ will vary in space and charges close to the barrier are reorganized, which results in a local change of the chemical potential $\mu_{\mathrm{c}}(r)$. This is schematically sketched in Figure 6.1. Expressing the charge as function of the chemical potential $\rho\left(\mu_{\mathrm{c}}(r)\right)$, the Poisson equation is: 


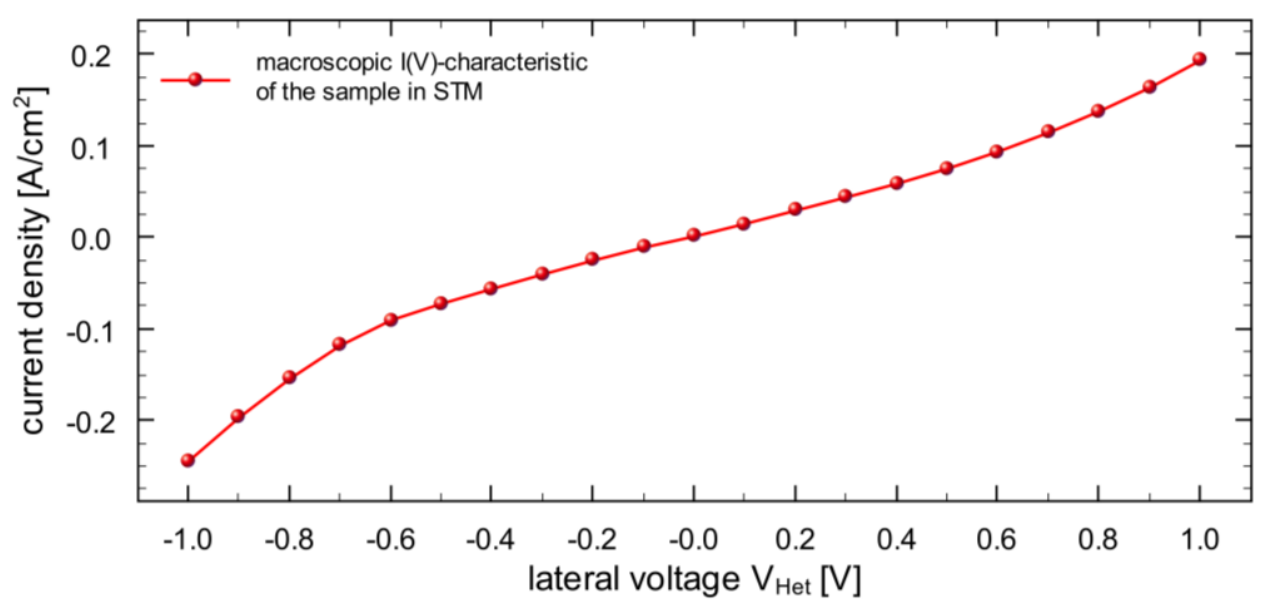

Figure 6.2: Macroscopic $I(V)$-characteristic of the heterostructure attached in the STM at $5 \mathrm{~K}$.

$$
\nabla^{2} V(r)=-\frac{\rho\left(\mu_{c}(r)\right)}{\varepsilon_{r} \varepsilon_{0}}=-\frac{\rho\left(\mu_{e c}+e V(r)\right)}{\varepsilon_{r} \varepsilon_{0}}
$$

Note that the electrochemical potential of the left side $\mu_{\mathrm{ec}}^{\text {left }}$ and the right side $\mu_{\mathrm{ec}}{ }^{\text {right }}$ of the AlAs barrier will be different if a lateral voltage $V_{\mathrm{Het}}$ is applied (compare Figure 6.1.). The equation for charge distribution is therefore shifted for the left and the right side by the applied voltage:

$$
\begin{gathered}
\nabla^{2} V(r)=-\frac{\rho\left(\mu_{e c}^{l e f t}+e V(r)\right)}{\varepsilon_{r} \varepsilon_{0}} \quad \text { (left side) } \\
\nabla^{2} V(r)=-\frac{\rho\left(\mu_{e c}^{r i g h t}+e V(r)\right)}{\varepsilon_{r} \varepsilon_{0}} \quad \text { (right side) }
\end{gathered}
$$

The number of charges $\rho\left(\mu_{\mathrm{c}}\right)$ as a function of the chemical potential is calculated for GaAs as well as for AlAs in Appendix A.2 and plotted in Figure A.11. In that calculation the GaAs conduction band edge minimum is defined to be zero energy. For a simulation the Poisson equation has to be solved self consistent, which is done by using the commercial program COMSOL Multiphysics.

In Figure 6.2 the current density as a function of the applied lateral voltage $V_{\text {Het }}$ is plotted for the sample attached to the STM at $5 \mathrm{~K}$. It is assumed that the current flows through the whole sample size which is $3 \times 3 \mathrm{~mm}^{2}$. The current is limited due to the AlAs barrier. As it was mentioned above the electrochemical potential $\mu_{\mathrm{ec}}$ is assumed to be constant on each side of the barrier. This is only true if the main voltage drop happens inside the AlAs barrier.

The measured current density through the AlAs barrier is about $0.1 \mathrm{Acm}^{-2}$ for a voltage of $0.5 \mathrm{~V}$. This voltage drop is compared to the expected voltage drop for a sample of homogeneous GaAs and the same current density. The current density can be expressed by the electron charge e, the number of charges $n$, the mobility $\mu$ and the electric field $E$. 


$$
j=q n \mu E
$$

As the sample is low doped in the interesting region the mobility for a doping concentration of $n=1 \cdot 10^{15} \mathrm{~cm}^{-3}$, which is at $5 \mathrm{~K}$ about $\mu=10^{4} \mathrm{~cm}^{2} / \mathrm{Vs}$ [92], is used. The sample thickness is $100 \mu \mathrm{m}$. With these values the expected voltage drop for a current density of $0.1 \mathrm{Acm}^{-2}$ is $0.6 \mathrm{mV}$, which is a factor of 800 smaller than the voltage drop at the AlAs barrier. Therefore the assumption that $\mu_{\mathrm{ec}}^{\text {left }}$ and $\mu_{\mathrm{ec}}^{\text {right }}$ is constant is valid.

\subsubsection{Extracting the potential from spectroscopy measurement}

This subsection should briefly introduce how the potential for different applied lateral voltages $V_{\mathrm{Het}}$ was extracted from the spectroscopy measurement.

As a first approximation the onset voltage for positive current is taken as a measure for the energetic position of the conduction band. An external potential would simply shift the position of the conduction band which can be measured by the shift in the onset voltage. The effect of TIBB as well as the effect of the band offset between the two materials GaAs and AlAs is assumed to be unchanged. The onset voltage for no applied lateral voltage $\left(V_{\mathrm{Het}}=0 \mathrm{~V}\right)$ is therefore taken as a reference. With this assumption the lateral resolved potential across the heterostructure can be obtained from spectroscopy by taking the difference of the onset voltage between an applied lateral voltage and zero lateral voltage.

As an example the above described method is presented in Figure 6.3 for a measured data set. Figure 6.3(a,b) shows two spectroscopy $I(x, V)$-sections at the same position for two lateral voltages. The black lines indicate the onset voltage of $V_{\mathrm{I}=1 \mathrm{pA}}$. In Figure $6.3(\mathrm{c})$ these lines are plotted in blue for $V_{\mathrm{Het}}=+0.3 \mathrm{~V}$ and in red for $V_{\mathrm{Het}}=-0.3 \mathrm{~V}$. Additionally, the reference onset voltage taken from a spectroscopy section (not shown here) for $V_{\mathrm{Het}}=0 \mathrm{~V}$ is plotted in dotted black as a reference. The difference between the lines with applied lateral voltage and the reference is shown in Figure $6.3(\mathrm{~d})$. The red line is the potential for $V_{\mathrm{Het}}=-0.3 \mathrm{~V}$ and starts approximately at $+0.1 \mathrm{~V}$ and ends at $-0.1 \mathrm{~V}$. The blue line is the potential for $V_{\mathrm{Het}}=+0.3 \mathrm{~V}$ and starts approximately at $-0.1 \mathrm{~V}$ and ends at $+0.1 \mathrm{~V}$. That means that the potential drop is about $0.2 \mathrm{~V}(-0.2 \mathrm{~V})$ for an applied lateral voltage of $0.3 \mathrm{~V}(-0.3 \mathrm{~V})$. In the lateral distance of $80 \mathrm{~nm}$, which is the lateral distance of the image, $65 \%$ of the applied lateral voltage drops across the heterostructure.

In the next paragraph the error of this analysis is estimated. One has to consider, if two spectroscopies with a different voltage setpoint, would also have different onset voltages. Figure 6.4(a) illustrates this difficulty. The Fermi energy of the tip $\mathrm{E}_{\mathrm{F} \text {,tip }}$ is sketched in dotted grey, the Fermi energy of the right side of the sample in dotted green. The voltage values correspond to the constant current topography used for the spectroscopy in Figure 6.3(a). The lateral voltage is $V_{\mathrm{Het}}=+0.3 \mathrm{~V}$. To the left $\operatorname{contact}^{7}$ a voltage of $-2.25 \mathrm{~V}$ is applied $\left(V_{\text {left }}=V_{\mathrm{Het}} / 2+V_{\mathrm{STM}}\right)$, to the right contact a voltage of $-2.55 \mathrm{~V}$ is applied $\left(V_{\text {right }}=-V_{\mathrm{Het}} / 2+V_{\mathrm{STM}}\right)$. The corresponding constant current topography image is taken at a voltage of $V_{\mathrm{STM}}=-2.4 \mathrm{~V}$. The "real" applied voltage is sketched by the black line. Due to the applied lateral voltage, the "real" voltage

\footnotetext{
${ }^{7}$ The left and right contacts correspond to the left and right side of the shown images. Compare Figure 5.2 .
} 

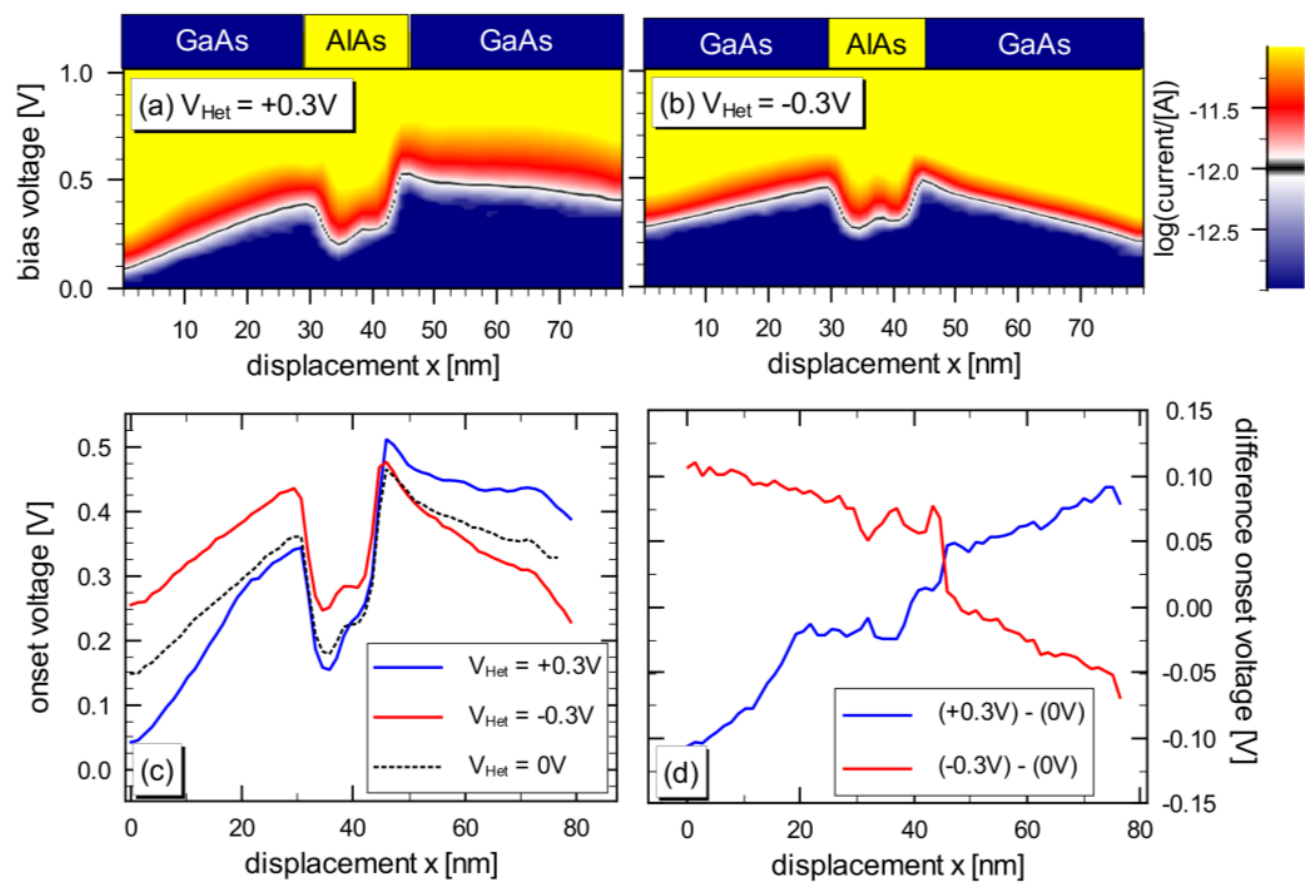

Figure 6.3: (a,b) Laterally resolved spectroscopy $I(x, V)$-section for two different lateral voltages $V_{\text {Het }}$ (a) $0.3 \mathrm{~V}$ and (b) $-0.3 \mathrm{~V}$. The black line shows a contour line of constant current $1 \mathrm{pA}$, the corresponding voltage is named onset voltage. In (c) the lateral resolved onset voltage is plotted for two lateral voltages $V_{\text {Het }}$ (red and blue) and the reference for no applied lateral voltage (dotted black). In (d) the difference of the onset voltage is plotted laterally resolved. It should give the voltage drop across the heterostructure.

setpoint changes in lateral direction. In this example it changes between $-2.25 \mathrm{~V}$ and $-2.55 \mathrm{~V}$.

For a laterally resolved spectroscopy measurement the topography has to be adjusted at each point. A different setpoint voltage means a different distance between tip and sample, which could lead to a shift of the spectroscopic feature. Therefore spectroscopies with voltage setpoints of $V_{\mathrm{STM}}=-2.25 \mathrm{~V}, V_{\mathrm{STM}}=-2.4 \mathrm{~V}$ and $V_{\mathrm{STM}}=-2.55 \mathrm{~V}$ all three having a lateral voltage of $V_{\mathrm{Het}}=0 \mathrm{~V}$ are taken and compared with each other, this is sketched in Figure 6.4(b). The shift of the spectroscopic feature due to the different voltage setpoints can be taken as error estimation.

The above explained error estimation is applied to a measured data set. In Figure $6.5(\mathrm{a}, \mathrm{b})$ laterally resolved spectroscopy $I(x, V)$-section for a setpoint of $V_{\mathrm{STM}}=-2.25 \mathrm{~V}$ and $V_{\mathrm{STM}}=-2.55 \mathrm{~V}$ are plotted logarithmically. The black lines indicate the onset voltage $V_{\mathrm{I}=1 \mathrm{pA}}$. In Figure $6.5(\mathrm{c})$ the onset voltage for three different setpoints are shown. The curves match quite well. The difference between the onset voltages of the setpoint of $V_{\text {STM }}=-2.25 \mathrm{~V}$ and $V_{\text {STM }}=-2.55 \mathrm{~V}$ (using the whole $I(x, y, V)$-spectroscopy data set) is plotted in Figure 6.5(d) as a histogram. The resulting difference can be determined by $\sim 22 \mathrm{mV}$, which is $7 \%$ of the difference of the voltage setpoints. 
(a)

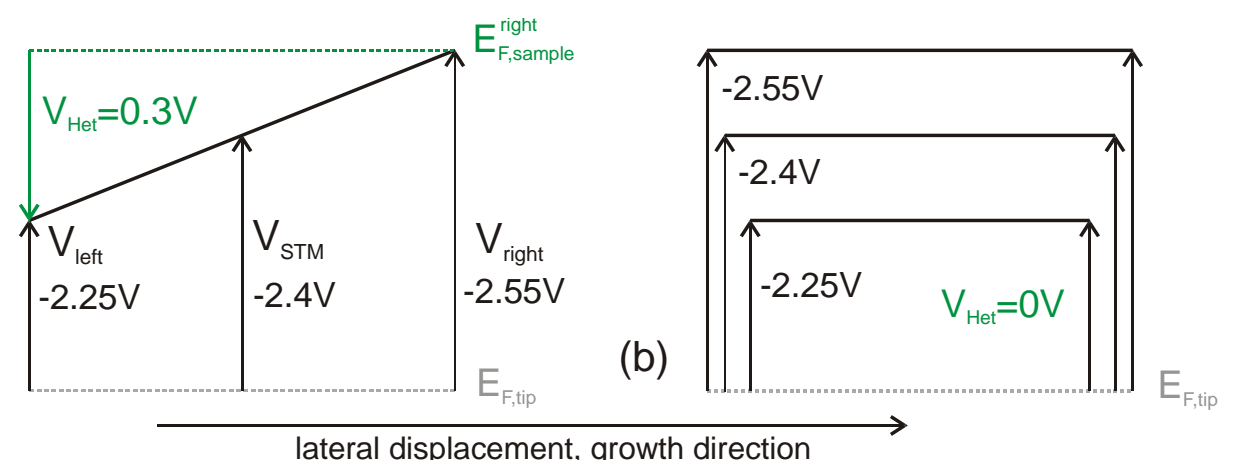

Figure 6.4: (a) The applied voltages on the left and right contacts for a lateral voltage of $V_{\text {Het }}=+0.3 \mathrm{~V}$. The Fermi energy of the tip is shown in dotted grey, the Fermi energy of the right side of the sample in dotted green. The "real" applied voltage changes in lateral direction (black line) (b) As a cross check, spectroscopies with different voltage setpoints are compared with each other.
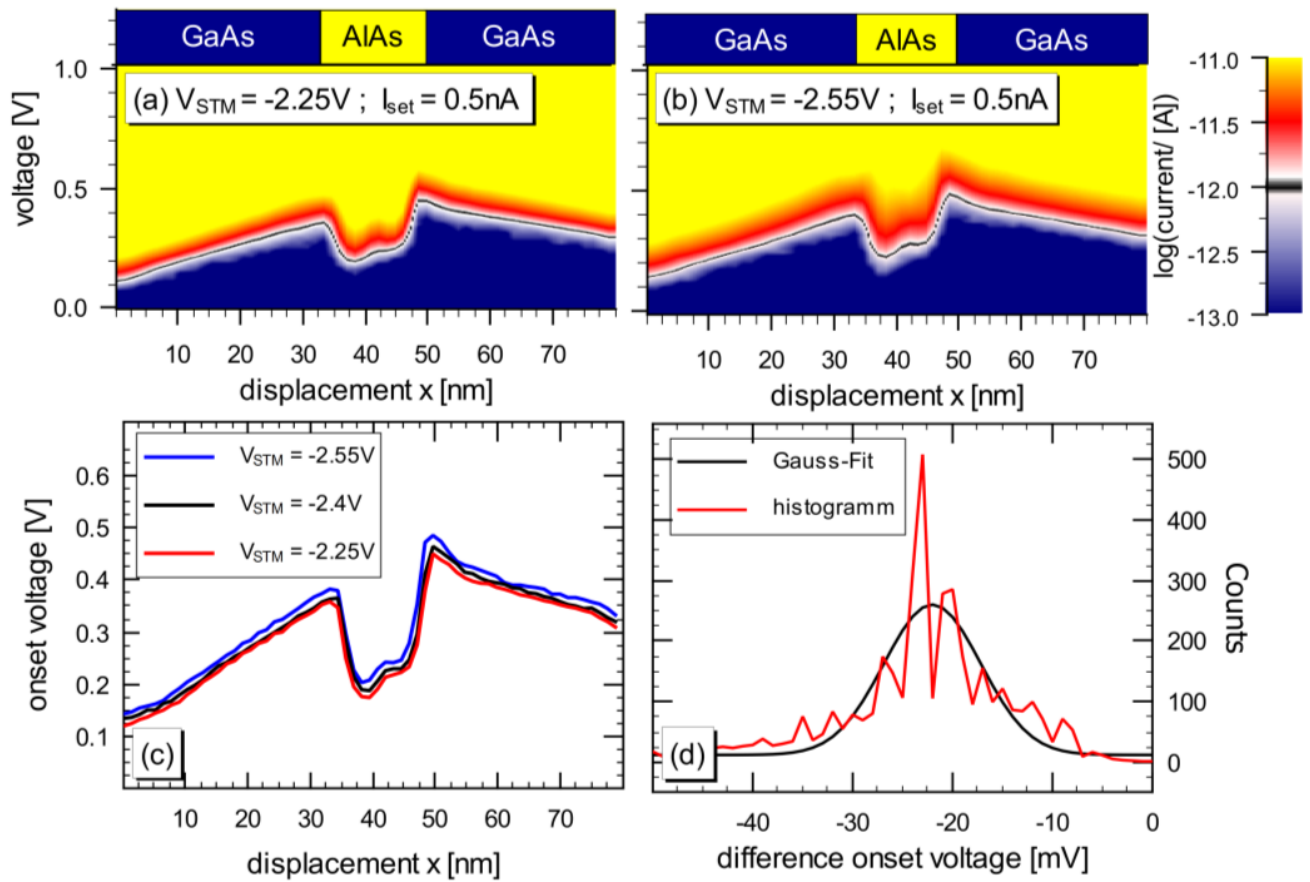

Figure 6.5: $(a, b)$ Laterally resolved spectroscopy $I(x, V)$-section for two different voltage setpoints, no lateral voltage $V_{\text {Het }}$ is applied. The black line shows a contour line of constant current $1 \mathrm{pA}$, the corresponding voltage is named onset voltage. In (c) the lateral resolved onset voltage is plotted for three different voltage setpoints. A histogram of the difference of the onset voltage (using two spectroscopies $I(x, y, V)$ for $V_{\text {STM }}=-2.25 \mathrm{~V}$ and $\left.V_{\text {STM }}=-2.55 \mathrm{~V}\right)$ is shown in (d) 


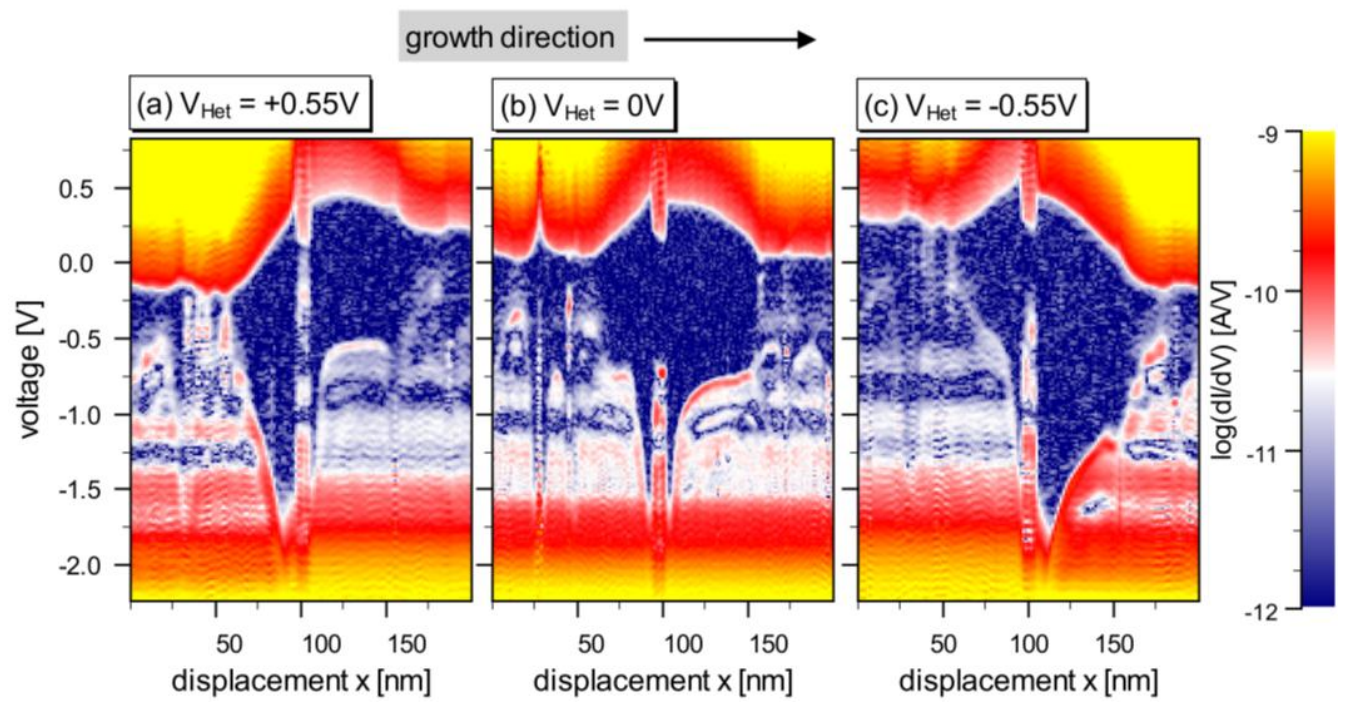

Figure 6.6: Differential conductivity $\mathrm{d} / / \mathrm{d} V(x, V)$-sections above the same position of the heterostructure for different lateral voltages (a) $V_{\mathrm{Het}}=+0.55$ (b) $V_{\mathrm{Het}}=0 \mathrm{~V}$ (c) $V_{\mathrm{Het}}=$ $-0.55 \mathrm{~V}$.

\subsubsection{The potential landscape in a biased RTD}

Three large scale $(200 \mathrm{~nm})$ differential conductivity $\mathrm{d} I / \mathrm{d} V(x, V)$-sections are shown in Figure 6.6. The sections are taken at the same position, for different lateral voltages (a) $+0.55 \mathrm{~V}$ (b) $0 \mathrm{~V}$ and (c) $-0.55 \mathrm{~V}$. The influence of the lateral voltage can clearly be seen, by the shift of the onset values left and right to the heterostructure. The onset voltage is extracted and plotted in Figure 6.7(a). It is almost constant at the edge of the plot (between $-100 \mathrm{~nm}$ and $-50 \mathrm{~nm}$, as well as between $+80 \mathrm{~nm}$ and $+100 \mathrm{~nm}$ ). The voltage has dropped completely in that area and the spectroscopy is like on homogeneous GaAs.

As mentioned above the voltage extracted for $V_{\mathrm{Het}}=0 \mathrm{~V}$ can be used as a reference for zero potential-drop across the diode. The difference between the onset voltage extracted for $V_{\mathrm{Het}}=-0.55 \mathrm{~V}$ and $V_{\mathrm{Het}}=0 \mathrm{~V}$ is taken, and showed as the blue crosses in Figure $6.7(\mathrm{~b})$. This curve corresponds to the potential landscape for the applied voltage $V_{\mathrm{Het}}=-0.55 \mathrm{~V}$. The red crosses show the difference of the onset voltage for $V_{\mathrm{Het}}=+0.55 \mathrm{~V}$ and $V_{\mathrm{Het}}=0 \mathrm{~V}$ and corresponds to the potential landscape for the applied voltage of $V_{\mathrm{Het}}=+0.55 \mathrm{~V}$. They are compared to the solution of the Poisson equation, which gives the potential landscape (solid lines) of the heterostructure. In the simulation the doping gradient (values like in Figure 4.2), the different materials with its different dielectric constants and the applied lateral voltage have been included. The used voltage drop across the heterostructure is not the full applied lateral voltage of $0.55 \mathrm{~V}$, but only $0.4 \mathrm{~V}$. This could be due to a voltage drop at the ohmic contacts of the sample. The voltage drop is not symmetrically, either. On the left side the voltage shift is $0.22 \mathrm{~V}$ and on the right side $0.18 \mathrm{~V}$. Thus, the ohmic contacts on the left and the right of the sample seem to be different.

In Figure 6.7(c) the charge distribution according to the simulation is shown. The screening of the potential drop is done by electrons from the conduction band and by the ionization of donors. The negative charge is located spatially close to the 

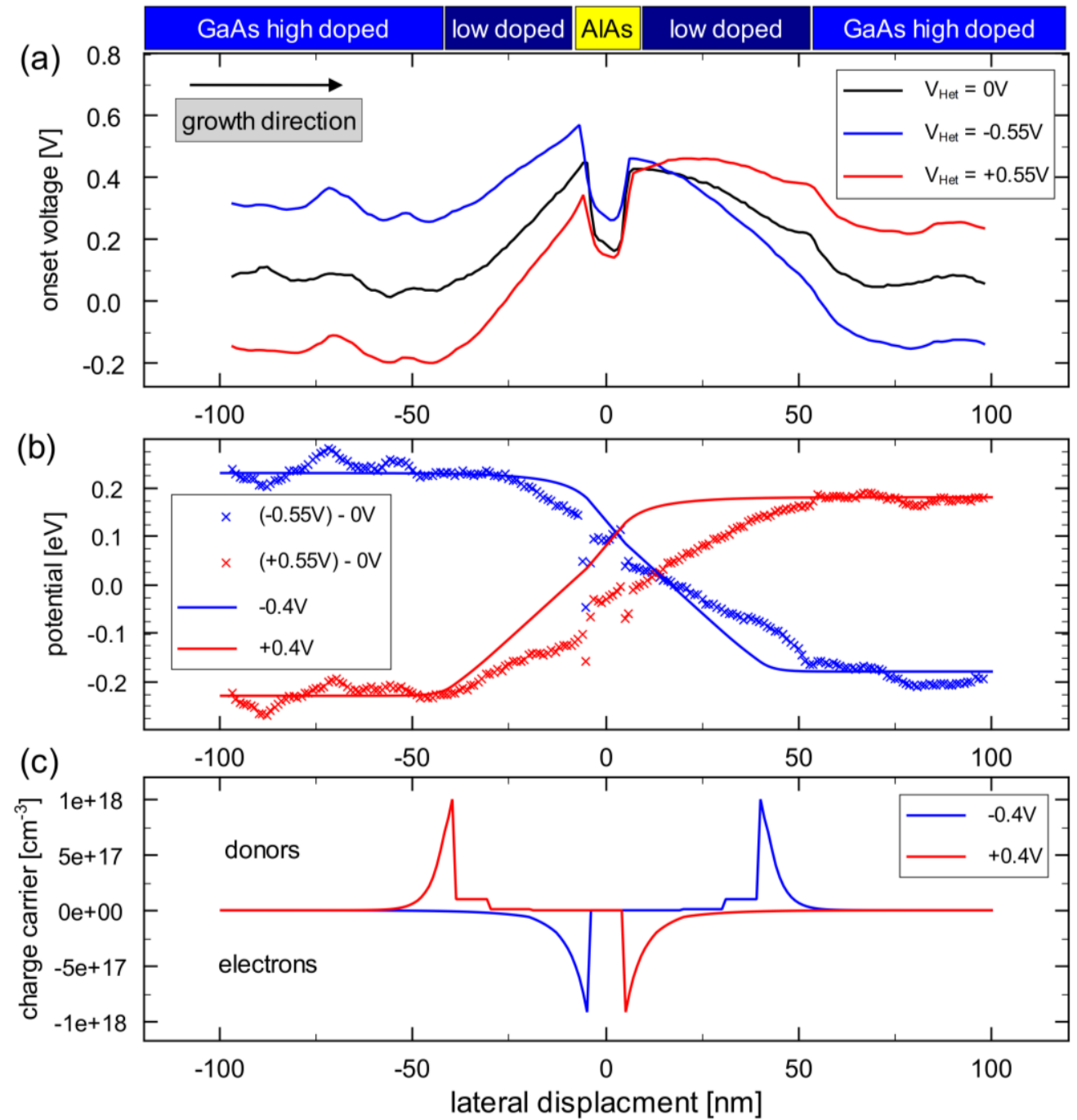

Figure 6.7: (a) The image shows the current onset voltage for positive voltage $V_{1=1 \mathrm{pA}}$, the influence of the lateral voltage is clearly visible. In (b) the difference of the onset value is plotted (crosses) and compared to the simulation of the potential across the heterostructure (solid lines) (c) charge carrier distribution according to the simulation.

heterostructure. The positive charges, which are caused by the ionization of donors, are located in the high doping area in $40 \mathrm{~nm}$ distance from the heterostructure. As the position of the positive and negative charges is different, the potential drop for negative and positive lateral voltage $V_{\mathrm{Het}}$ should be located at different positions.

For a better visualization the potential for positive voltage (red crosses and red line) is multiplied by a factor of -1 . The result is shown in Figure 6.8(a). In the simulation a spatial shift of the potential for positive and negative lateral voltage is clearly visible. This shift is also visible in the data points but here it is not that much extended. Furthermore the mean value of the potential for positive and negative lateral voltage is calculated (grey crosses and grey line). The slope of the simulated potential and the data points are slightly different.

In the simulation the doping gradient on the left and right side of the sample was assumed to be symmetrical. This is according to the growth process of the GaAs 

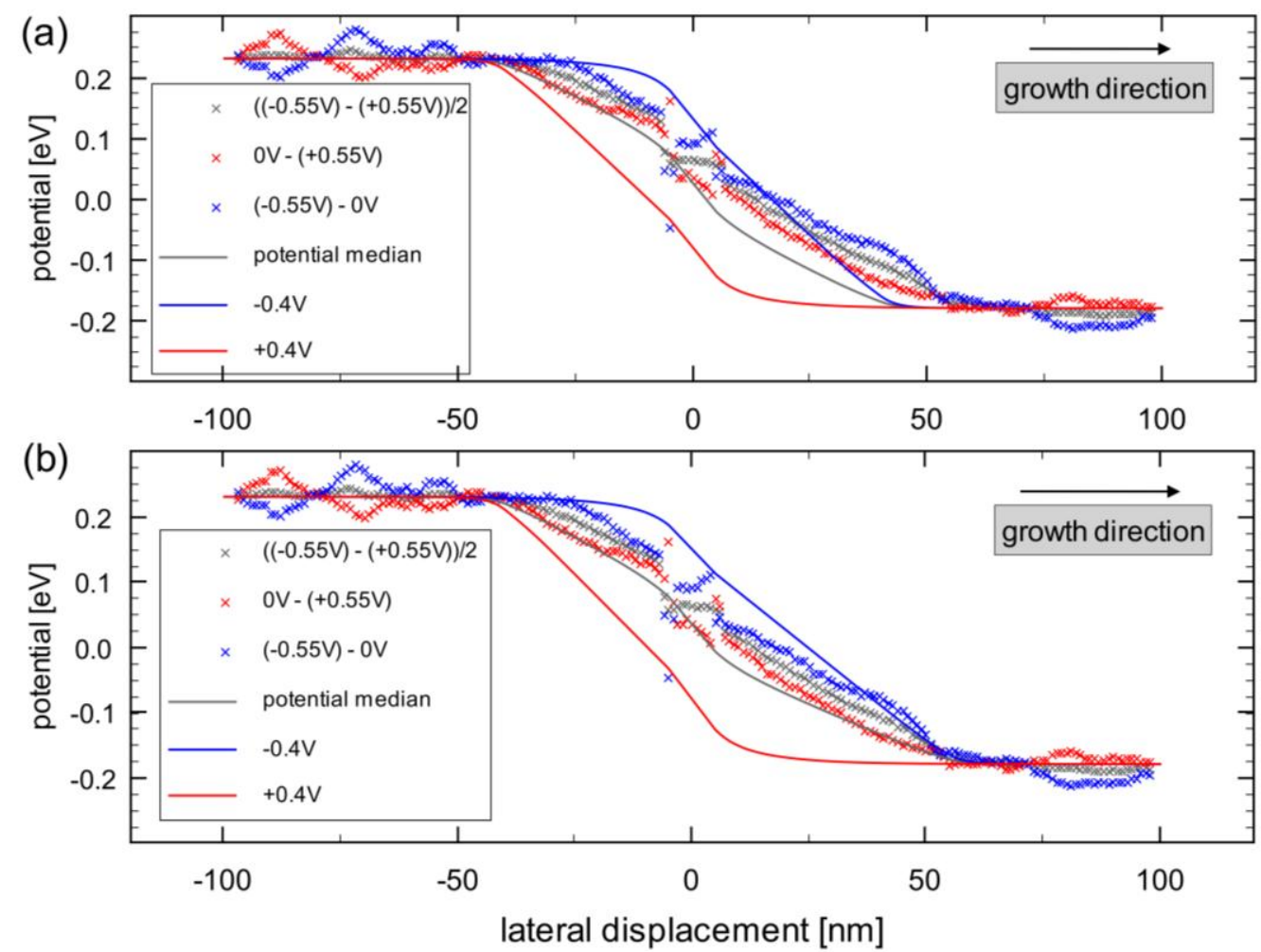

Figure 6.8: Comparison of the measurements to the simulation. For a better visualization the potential for positive lateral volage (red curve) is multiplied with -1 (compare Figure 6.7(b)). (a) the intrinsic GaAs layer is symmetrically $15 \mathrm{~nm}$ on both sides, (b) the intrinsic GaAs layer is $15 \mathrm{~nm}$ on the left side and $30 \mathrm{~nm}$ on the right side.

layers. In large scale topographical measurements, one can extract that the doping is slightly asymmetrical (compare Figure A.23). An asymmetrical doping has also been reported for the specific sample by the transport measurements across the resonant tunneling diode [70]. Thus, the simulation was modified according to the topographical observation: The intrinsic layer of GaAs has been extended in growth direction (right side) to $30 \mathrm{~nm}$ instead of $15 \mathrm{~nm}$. It is shown in Figure 6.8(b). A better result with the data is obtained for the potential median. But still the shift between the curves for positive and negative lateral voltage is larger in the simulation.

A possible explanation for this discrepancy is that the positive and negative charges are both located in the same distance from the heterostructure. Then the potential for negative and positive lateral voltage would be symmetrical and no lateral shift is expected. One possible reason is that the bands at zero lateral voltage are not flat, like it was expected for the simulation. If they are enhanced in the vicinity of the AlAs layer, the negative charge would accumulate more far away from the AlAs layer. The enhancement of the bands could be due to a positive charge on the AlAs layer, or due to the different work functions between GaAs and AlAs. 

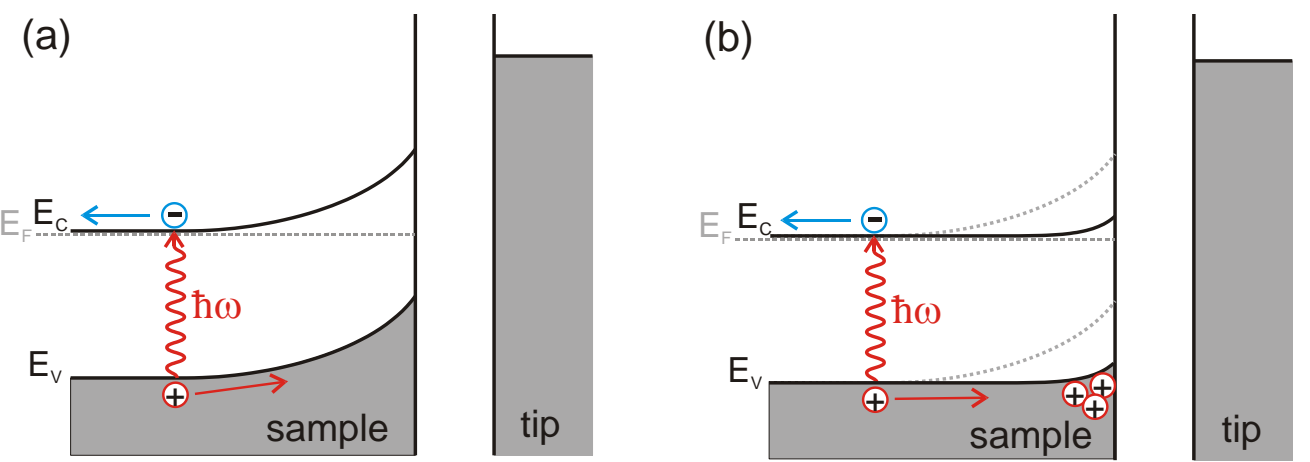

Figure 6.9: The image shows a sketch of the energy band diagram under laser radiation in z-direction. (a) If the laser is switched on electron (-) hole (+) pairs are generated. As the positive tip-sample voltage causes a positive tip induced band bending, the generated electrons are rejected from the surface and the holes are accumulated at the surface. (b) The positive charge of the holes screens the potential of the tip and the tip induced band bending is reduced.

\subsection{Direct measuring of the band edge profile by optical excitation}

\subsubsection{Motivation}

The principle idea of the spectroscopy of GaAs with light excitation is to reduce the effect of tip induced band bending (TIBB). The TIBB makes it difficult to extract the band edge profile directly from the spectroscopy measurement, especially as there is a donor gradient inside the sample. As the screening due to the ionization of donors is small in the undoped GaAs area the influence of the TIBB is expected to be large. The aim of the laser radiation is to create almost the same amount of holes in the low doped region like the amount of donors in the high doped region. Then the screening and also the TIBB should be similar and the onset values of the two regions are comparable.

Figure 6.9 illustrates the idea of the measurement. The used laser energy is $1.58 \mathrm{eV}$, which is slightly larger than the band gap in GaAs $(1.52 \mathrm{eV}$ at $5 \mathrm{~K})$. Thus, it is possible to create electron hole pairs. Due to the tip induced potential gradient, the electrons are repelled from the tip, which means they are repelled from the surface and the holes are accumulated to the surface underneath the tip. The positive charge underneath the tip screens the potential similar to the case of ionized donors. Thus, the bands at the surface will be flat (see Figure 6.9(b)). The onset value of the low and the high doped GaAs area are comparable. 

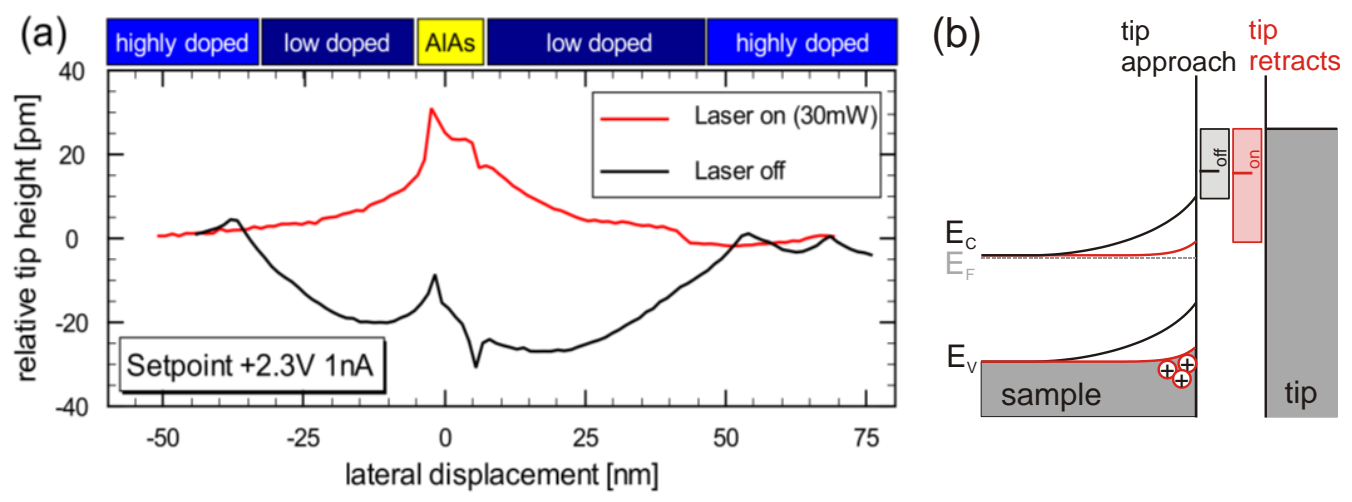

Figure 6.10: (a) Constant current topography sections taken at a setpoint of $+2.3 \mathrm{~V}$ and $1 \mathrm{nA}$ without laser radiation (black line) and with a laser intensity of $30 \mathrm{~mW}$ (red line). (b) The corresponding energy band diagram in the low doped area is shown. The amount of tunnel current for the same voltage and tip distance is visualized by the colored squares in the vacuum barrier for the case of no laser radiation $\left(\mathrm{I}_{\text {off }}\right)$ and with laser radiation $\left(\mathrm{I}_{\mathrm{on}}\right)$.

\subsubsection{Topographical investigation}

As a first check if the excited laser light is doing the desired effect, two constant current topography images at the same position and voltage setpoints are compared. A topographic height section perpendicular to the heterostructure is shown in Figure 6.10(a). For the topography without laser irradiation (black) the TIBB effect is present, and the tip has to approach the sample in the low doped area. For the topography with a laser intensity of $30 \mathrm{~mW}$ (red) the TIBB effect is screened and the topographic height stays almost constant on the low doped GaAs. The reason is schematically sketched in Figure 6.10(b). The amount of current expected for the same tip-sample distance is indicated by the colored squares in the vacuum barrier for the case of no laser radiation $\left(\mathrm{I}_{\text {off }}\right)$ and with laser radiation $\left(\mathrm{I}_{\mathrm{on}}\right)$. A topography image is adjusted to constant current. This means if the current is too small, the tip will approach to the surface and if the current is too large the tip will retract from the surface. This is confirmed in the measurement.

\subsubsection{Band edge profile under laser radiation}

For different laser intensities the onset voltage $V_{\mathrm{I}=5 \mathrm{pA}}$ is extracted by using five $I(x, V)$ spectroscopies taken at the same position. For this analysis the current defining the onset voltage is chosen to be 5pA. The result is shown in Figure 6.11. As expected the effect of the laser light is visible in the low doped area of GaAs, whereas in the high doped area the onset voltage stays almost constant. Even for the highest laser intensity of $30 \mathrm{~mW}$ the bands are not completely flat. This could be a hint that the band edge profile close to the AlAs barrier is enhanced.

In the following paragraph an estimation of the amount of holes which are used for screening is done: For a photon energy of $1.58 \mathrm{eV}$ the laser power of $1 \mathrm{~mW}$ corresponds to $3.9 \cdot 10^{15}$ photons/s. The absorption coefficient in GaAs for an energy of $1.6 \mathrm{eV}$ is $1.48 \cdot 10^{4} \mathrm{~cm}^{-1}$ [93], which corresponds to a penetration depth of $474 \mathrm{~nm}$. The used laser spot is unfocussed. Having a look at the data one can see that for $0.75 \mathrm{~mW}$ 


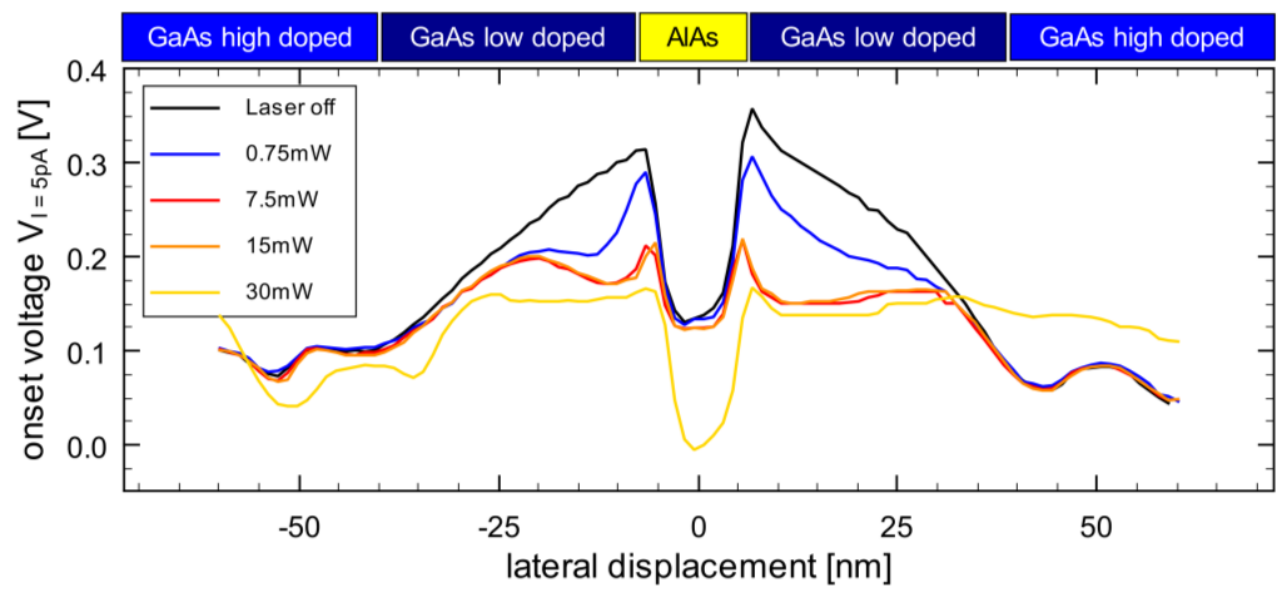

Figure 6.11: Positive onset voltage $V_{l=5 \mathrm{pA}}$ for different laser intensities. The higher the intensity the more the tip induced band bending is screened and the current starts at lower voltage.

\begin{tabular}{l|l}
\hline \hline $\begin{array}{l}\text { laser intensity } \\
{[\mathrm{mW}]}\end{array}$ & $\begin{array}{l}\text { hole density } \\
{\left[\mathrm{cm}^{-3}\right]}\end{array}$ \\
\hline 0.75 & $1 \cdot 10^{16}$ \\
7.5 & $1 \cdot 10^{17}$ \\
15 & $2 \cdot 10^{17}$ \\
30 & $4 \cdot 10^{17}$ \\
\hline \hline
\end{tabular}

Table 2: Expected hole density, which is valid for the screening of the tip, for different laser intensities.

already a screening effect is visible. This means that for this intensity the amount of holes is larger than the intrinsic doping concentration. Let us assume that this concentration is $1 \cdot 10^{16} \mathrm{~cm}^{-3}$. For an unfocussed laser spot this would correspond to a hole life time in the order of $100 \mu \mathrm{s}$. Comparing this value with the free exciton life time, which is reported to be a few ns [94], the value seems large. But as the electrons and holes are separated spatially similar like in a solar cell, the enhancement of the value is reasonable. The hole density for the higher intensities using the above described estimation is summarized in Table 2:

According to the estimation, the laser intensity of $30 \mathrm{~mW}$ creates almost the same amount of holes, like the doping concentration in the high doped area. This means that the screening in the high and low doped area should be almost the same if the laser is aimed on the sample. Then, the effect of TIBB should be similar in both regions. The onset value should therefore give the band edge profile at the heterostructure. As one can see in Figure 6.11 the band edge profile in the low doped area is enhanced compared to the high doped area. 

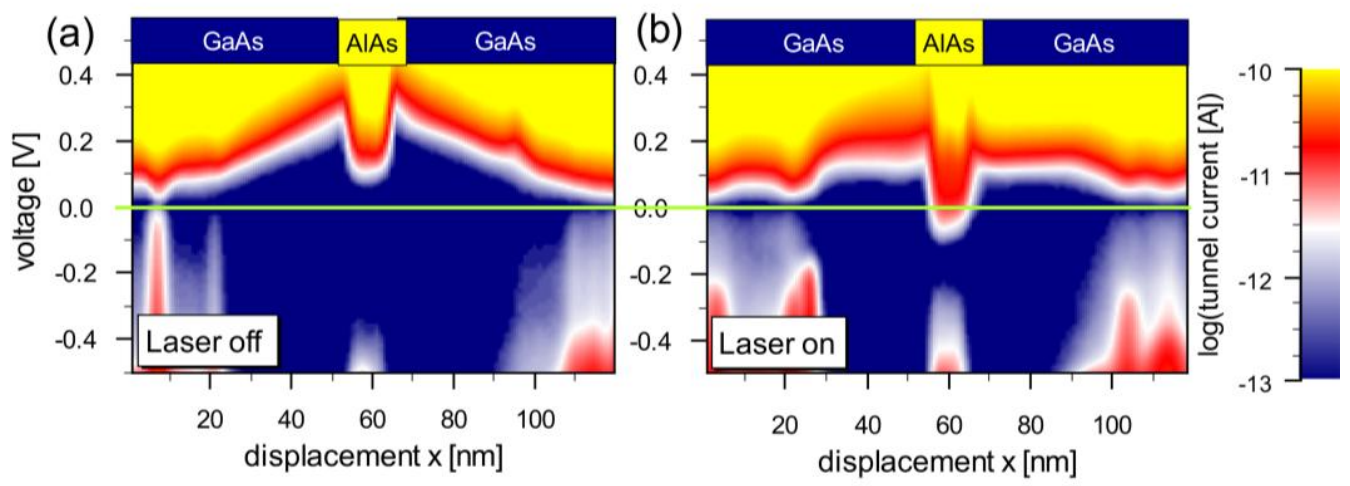

Figure 6.12: $I(V, x)$-sections at the same position for (a) Laser off (b) Laser on (30mW). On the AIAs also current at $\mathrm{OV}$ is measurable. The corresponding topography images are taken at $-2 \mathrm{~V}$ and $1.2 \mathrm{nA}$.

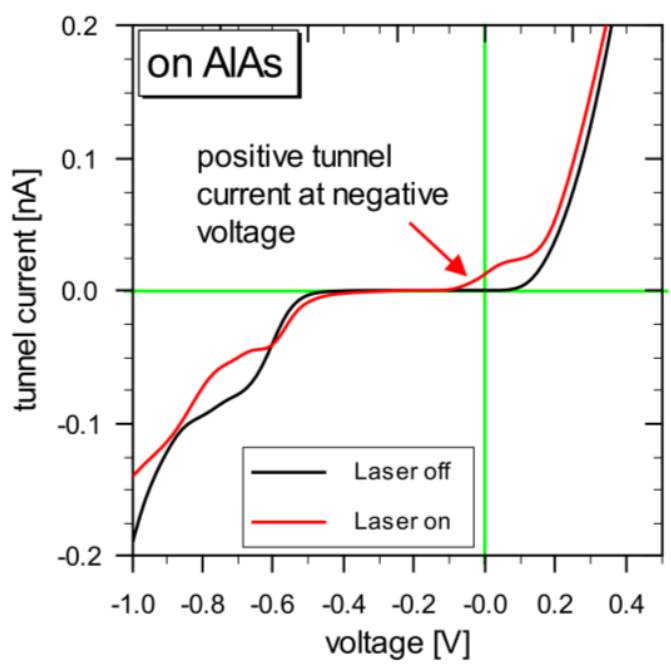

Figure 6.13: $/(V$-spectrum taken on AlAs with an laser intensity of $30 \mathrm{~mW}$ (red) without laser radiation (black). The laser radiation induces positive tunnel current at negative voltage.

\subsubsection{Outlook: The photo induced current}

The main purpose of the laser excitation was the investigation of the band edge profile, which was described in the previous subsection 6.2.3. It was found that the bands close to the AlAs are most likely enhanced. Another interesting phenomenon occurred due to the laser radiation: The laser excitation also leads to photo induced current. This subsection is meant as an outlook, where this observation is described and a brief possible explanation of that phenomenon is suggested.

In Figure 6.12 two current $I(x, V)$-sections for (a) no laser radiation and (b) a laser intensity of $30 \mathrm{~mW}$ are shown. The green line shows the line of zero tip-sample voltage. At the position of AlAs the laser radiation causes a positive current at negative tip-sample voltage. This is emphasized in an $I(V)$-curve in Figure 6.13, where 


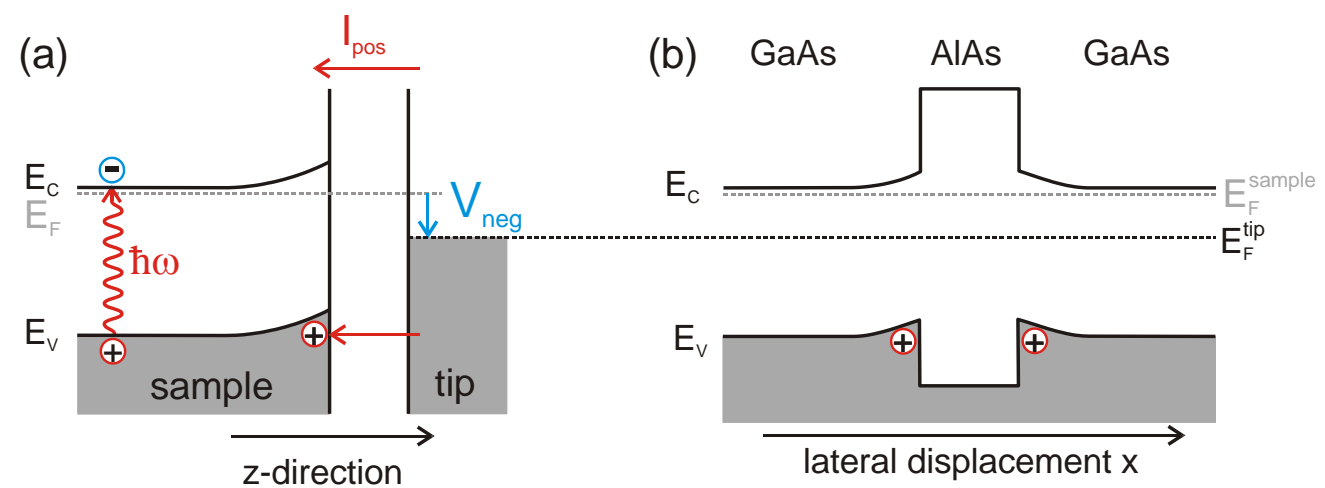

Figure 6.14: Possible explanation for the positive current at negative voltage. (a) Energy band diagram perpendicular to the surface, holes accumulate at the enhanced potential and can tunnel from the sample into the tip, which causes a positive current. (b) Energy band diagram parallel to the surface.

the current on AlAs is compared for the case of no laser radiation (black) and with laser radiation (red).

In Figure 6.14(a) a possible explanation is shown. As the current is positive for negative sample voltage, electrons have to tunnel from the tip into the sample. As no states in the tip are available in the energy range of the conduction band, the electrons have to tunnel into the valence band. This current direction is possible if the bands at the surface are enhanced and holes can accumulate in that region. Then, electrons can tunnel from the filled states of the tip into the accumulated holes and a positive current at a negative voltage would occur. Figure 6.14(b) shows the energy band diagram laterally resolved. As the valence band maximum in GaAs is expected to be larger in energy than in AlAs, the holes would not accumulate in the region of the AlAs. This is in contradiction to the measurements, as the photo induced current is observed on the AlAs layer. One possibility is that the holes accumulate close to the AlAs layer and the tunneling process is done via $\mathrm{X}$-band states in the AlAs into the GaAs layer.

Up to now, the explanation is meant as a suggestion for the observed phenomen. Further studies would have to show, if this explanation is valid. A possible experiment would be to investigate a sample having a larger AlAs barrier. Then the photo induced current should only occur at the edge of the AlAs barrier. 


\section{Tip induced states in low doped GaAs}

The tip induced state is investigated in an undoped area of a heterostructure. The low doping concentration allows studying the tip induced state without any perturbation of surrounding donors. An AlAs barrier inside the undoped region influences the tip induced state resulting in an energetic shift of the state. This shift is compared with simulations of the potential landscape of the tip-heterostructure-system.

\subsection{Motivation}

The states which are formed in a tip induced quantum dot on III-V semiconductors have been investigated in InAs by Dombrowski et al. [18] and in GaAs by Wenderoth et al. [19] and by Feenstra et al. [95]. The tip induced quantum dot has been investigated by electroluminescence spectra by Croitoru et al. [96].

Due to the tip induced band bending (TIBB) the conduction band is shifted below the Fermi energy for negative tip-sample voltage. The induced potential landscape is called tip induced quantum dot. If the quantum dot is large enough, a bound state can exist, which is called tip induced state. As scanning tunneling microscopy on homogeneous undoped GaAs is difficult, in investigations up to now this state was always disturbed by the surrounding donors. The TIBB is too large on those samples and it is difficult to obtain a tunnel current without laser illumination [97]. In this chapter the low doping area of the heterostructure sample is used to investigate the tip induced state. The AlAs barrier inside the low doped GaAs region modifies the energetic position of the state. The dependency of the lateral distance to the barrier on the energetic position of the state is investigated.

In Figure 7.1 a differential conductivity $\mathrm{d} I / \mathrm{d} V(x, V)$-section at negative voltages is shown. A tip induced state is clearly visible in the low doped area, in a voltage range from about -0.7 to $-1.3 \mathrm{~V}$ depending on the distance to the AlAs barrier. For smaller voltage no differential conductivity is visible. With decreasing tip distance to the 


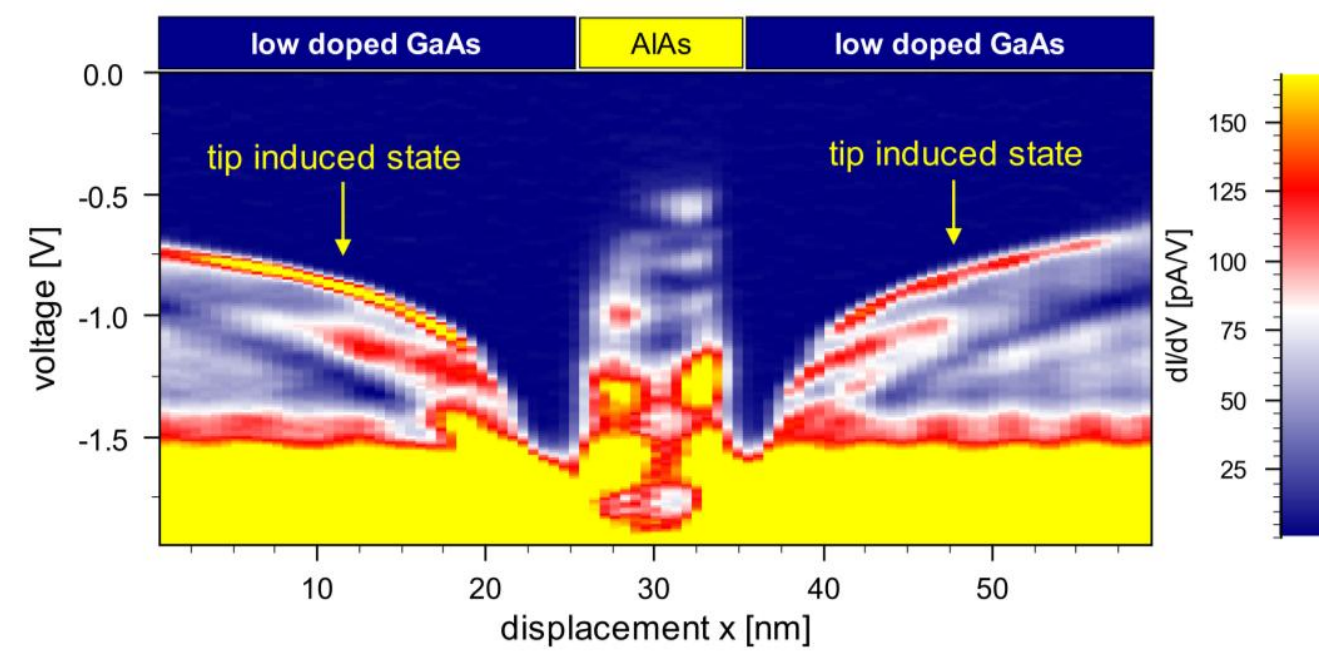

Figure 7.1: Differential conductivity $\mathrm{d} / / \mathrm{d} V(x, V)$-section for negative voltage. On the low doped GaAs area a peak is visible, which is shifting to higher negative voltages by approaching to the AIAs layer.
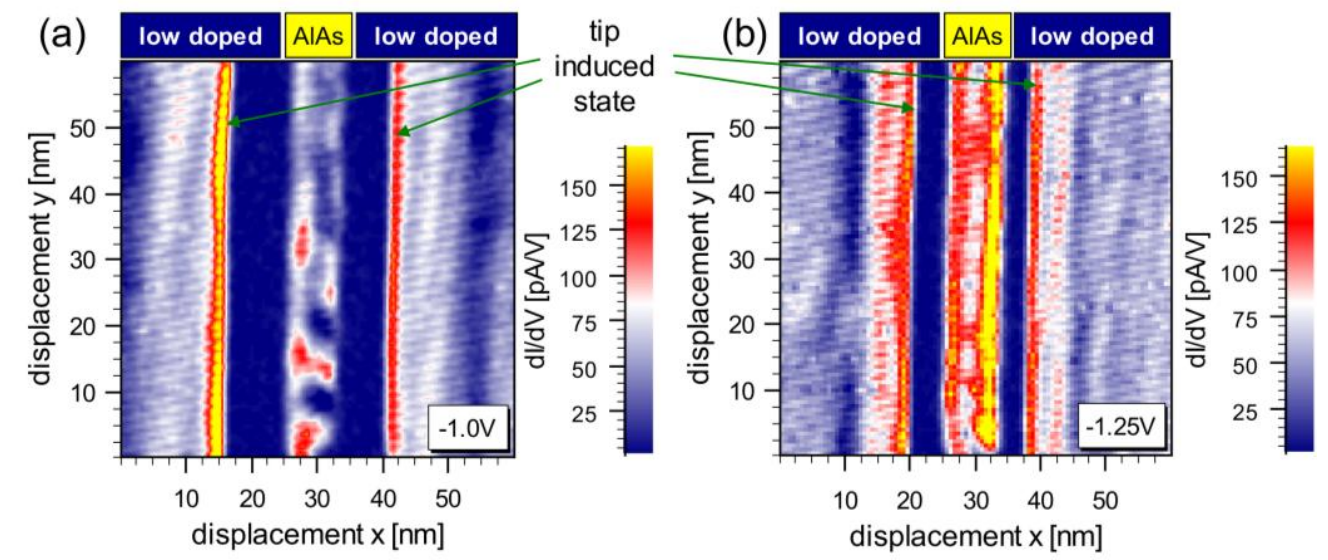

Figure 7.2: Differential conductivity $\mathrm{d} / / \mathrm{d} V(x, y)$-maps for (a) $-1.0 \mathrm{~V}$ and (b) $-1.25 \mathrm{~V}$. The tip induced state (indicated by the green arrow) is visible parallel to the AlAs barrier, and is not disturbed by any dopant atom.

heterostructure the state is shifted to higher negative voltage. For two voltages of $-1 \mathrm{~V}$ and $-1.25 \mathrm{~V}$ a differential conductivity $\mathrm{d} I / \mathrm{d} V(x, y)$-map is shown in Figure 7.2. In comparison to the state occurring on high doped GaAs (compare Figure A.7) this state is not disturbed by any surrounding donor. Instead the state is visible parallel to the AlAs barrier.

In subsection 7.1.1 it will be verified that it is reasonable that this peak is a tip induced state. Section 7.2 deals with the question, why the tip induced state shifts to higher negative voltage with decreasing distance to the AlAs barrier. The last section 7.3 will show how the tip induced state reacts on the potential of an applied lateral voltage. 


\subsubsection{Verification of the tip induced state}

For the identification of the tip induced state an atomic resolved spectroscopy measurement in the low doped area of GaAs was taken. In subsection 1.2.2 it was described that the atomic corrugation of the conduction band states (visible at positive voltage) and the valence band states (negative voltage $>-1.5 \mathrm{~V}$ ) are shifted spatially, as the maxima are located on either the gallium sublattice or on the arsenic sublattice. Using an atomically resolved spectroscopy, one can compare the corrugation at a voltage in the conduction band and at a voltage in the valence band with the corrugation of the tip induced state. If the corrugation of the tip induced state is more conduction band like, it is a hint, that the states have mainly conduction band character. This investigation was done in Figure 7.3, where a highly resolved spectroscopy is shown. A spectroscopy measurement is stabilized at a constant current topography image. In this image usually the atomic corrugation is visible, which means that the distance between tip and sample varies in lateral space. If the tip is close to the surface the current will be larger compared to the situation that the tip is in a larger distance to the surface. To compare the current between two lateral positions, the spectroscopy is normalized to a flat plane to avoid crosstalk from the constant current topography image. The normalization is done by simultaneously measuring the apparent barrier height, which is a measure for the distance dependent current (see

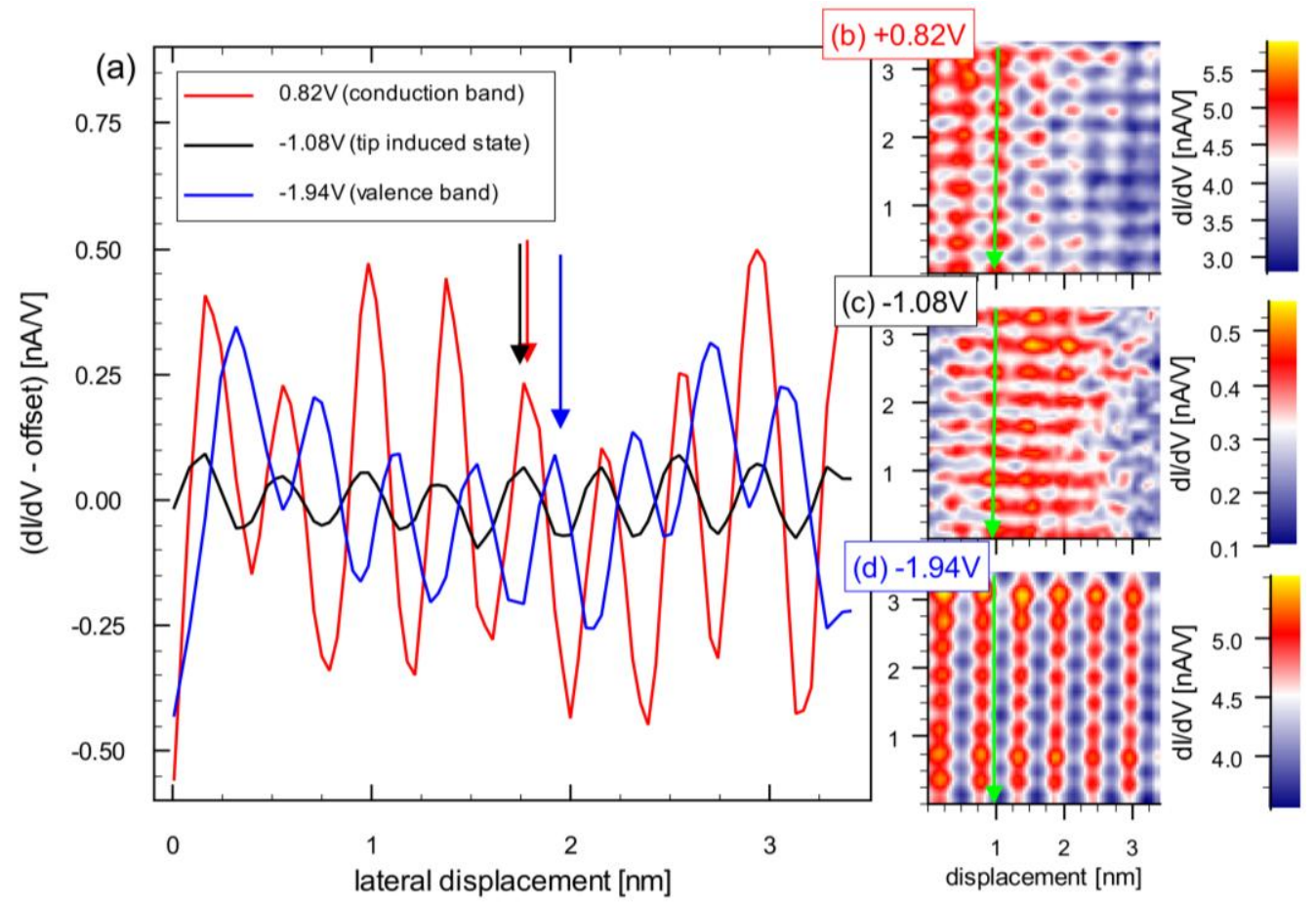

Figure 7.3: Identification of the tip induced state: A differential conductivity measurement was taken in the low doped area of GaAs. Image (a) shows differential conductivity $\mathrm{d} / / \mathrm{d} V(y)$-curves at three voltages. At $+0.82 \mathrm{~V}$ (red) the corrugation of the conduction band is visible, at $-1.94 \mathrm{~V}$ (blue) the corrugation of the valence band is visible. At the voltage of the tip induced state $-1.08 \mathrm{~V}$ (black) the corrugation is conduction band like (compare the maxima indicated by the arrows). (b-d) atomically resolved differential conductivity $\mathrm{d} / / \mathrm{d} V(x, y)$-maps at these voltages are shown. (The corresponding topography was taken at a setpoint of $-2.0 \mathrm{~V}$ and $1 \mathrm{nA}$.) 
subsection 2.2.4)

In Figure 7.3(a) the differential conductivity $\mathrm{d} I / \mathrm{d} V(y)$ as a function of displacement $y$ is plotted for three different voltages: At $+0.82 \mathrm{~V}$ (red) the conduction band states are mapped, at $-1.08 \mathrm{~V}$ (black) the tip induced state is mapped and at $-1.94 \mathrm{~V}$ (blue) the valence band states are mapped. For having better contrasts in each curve an offset line was removed. The corrugation maxima are indicated by the arrows. The maxima for the conduction band states and the tip induced state coincide whereas the maxima for the valence band are shifted. The Figure 7.3(b-d) show the laterally resolved differential conductivity $\mathrm{d} I / \mathrm{d} V(x, y)$-maps at these voltages. The green line indicates the position of the $\mathrm{d} I / \mathrm{d} V(y)$-section. From this measurement it is most likely that the state occurring at $-1.08 \mathrm{~V}$ has conduction band character.

\subsection{Potential landscape of the tip induced state}

In a three dimensional potential $V(\boldsymbol{r})$ does not necessarily exist a bound state. The extension of the potential in energy as well as in space has to be large enough for the formation of a state (see for example [50]). This is also true for the tip induced quantum dot, which is a potential caused by the TIBB. For low negative tip voltage the extension of that potential in energy and in space is small, and the potential enlarges by increasing the applied negative voltage. At a certain voltage the potential is large enough and a state can be formed. For homogeneous GaAs the extension of the tip induced quantum dot is dependent on the voltage as well as on the geometry of the tip.

In this section the tip induced state is investigated close to an AlAs barrier. Due to that barrier the extension of the tip induced potential is reduced. The reduction is larger, for smaller distances between tip and the AlAs barrier. The potential landscape of the tip induced quantum dot close to an AlAs barrier is simulated in three dimensions. The doping concentration, the AlAs barrier as well as the difference of the work functions for GaAs and AlAs are included. It is described in more detail in section A.2. A crosssection through the simulated potential landscape for a fixed voltage $(-0.5 \mathrm{~V})$ is shown in Figure 7.4. In Figure 7.4(a) the tip is 30nm away from the AlAs barrier. If one assumes that the voltage is just large enough to form a bound state, one can use this potential landscape as a reference. This landscape can be compared to the potential landscape for different tip distances to the AlAs barrier. In Figure 7.4(b) the tip is closer to the AlAs barrier (10nm). The lateral extension in the potential landscape has decreased. This is more dramatically visible in Figure 7.4(c); here the tip is only $2 \mathrm{~nm}$ away from the AlAs barrier. As the lateral extension of the potential landscape is important to form a state the potential of Figure 7.4(b) and (c) would be too small to create a bound state. To compensate the lateral shrinking the energetical amount of the potential would have to be increased. This is possible by applying a higher negative voltage. 


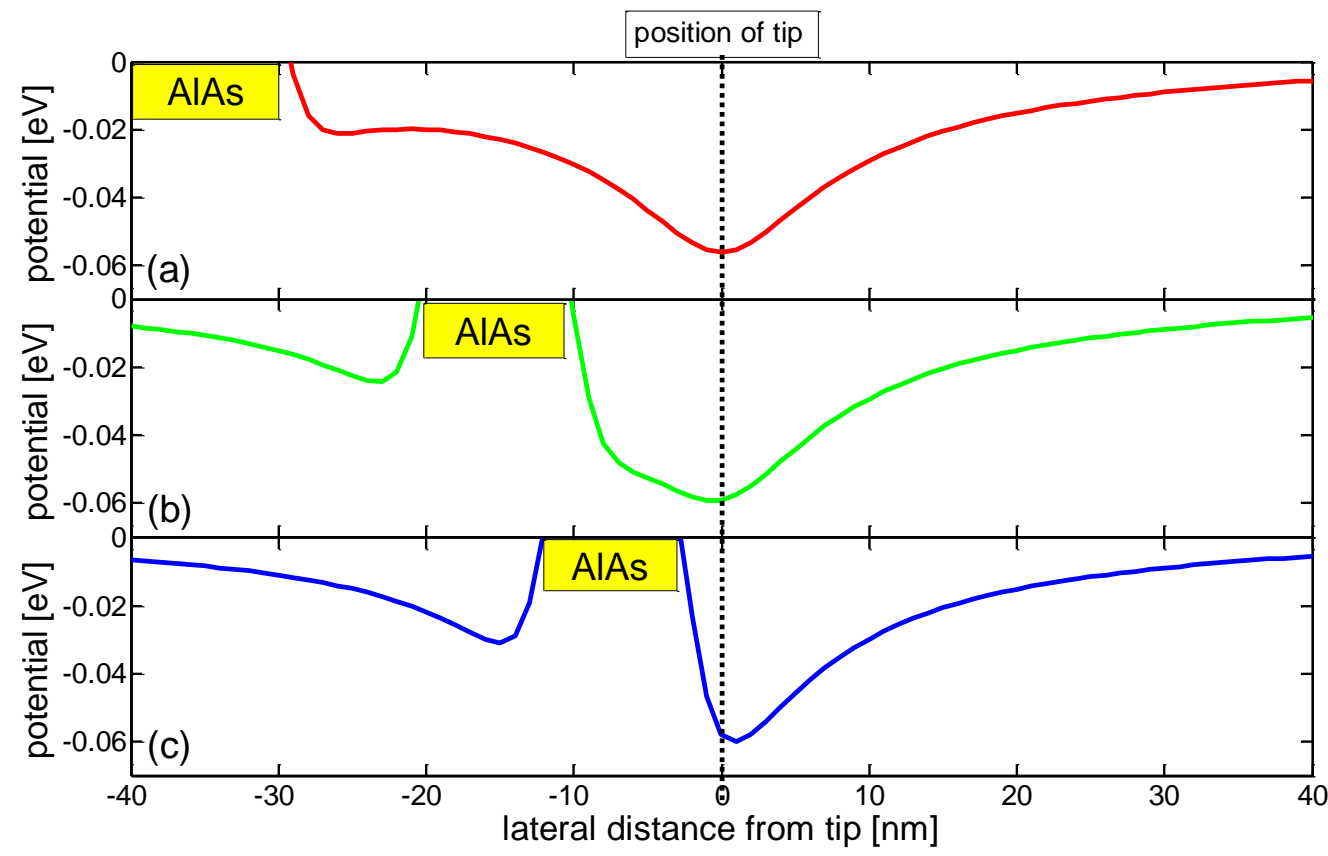

Figure 7.4: Potential landscape at the surface for an applied voltage of $-0.5 \mathrm{~V}$. In this simulation different work functions on AIAs and GaAs are assumed. The position of the tip is at $0 \mathrm{~nm}$, the distance of the tip to the AlAs barrier is (a) $30 \mathrm{~nm}$ (red) $10 \mathrm{~nm}$ (green) and $2 \mathrm{~nm}$ (blue). For a larger distance from the AlAs layer, the tip induced quantum dot is extended more laterally, and a state could form at a smaller voltage.

This is in accordance with the measurement, which can be seen in Figure 7.1: with decreasing tip distance to the AlAs barrier, a peak occurs in the differential conductivity for larger negative voltage.

\subsection{The influence of the lateral voltage on the tip induced state}

In this section the influence of the lateral voltage on the tip induced state is investigated. It will turn out that the onset voltage of the tip induced state is linked to the potential profile of the biased heterostructure.

The expected behavior is plotted in Figure 7.5. In Figure 7.5(a) the conduction band edge profile in GaAs across the heterostructure is sketched for no applied lateral voltage as the black solid line and for a voltage drop of $+0.4 \mathrm{~V}$ as the red solid line. (As it was shown in subsection 6.1.2 this voltage drop would correspond to a "real" applied voltage of $+0.55 \mathrm{~V}$. This is why later in the text the measurements will be labeled as $+0.55 \mathrm{~V})$. Outside the voltage drop, the measurement should behave like homogeneous GaAs, but shifted by $-0.2 \mathrm{~V}$ (left) and $+0.2 \mathrm{~V}$ (right). Due to the measurement technique the onset voltage of the tip induced state at that position should be shifted by that amount. 


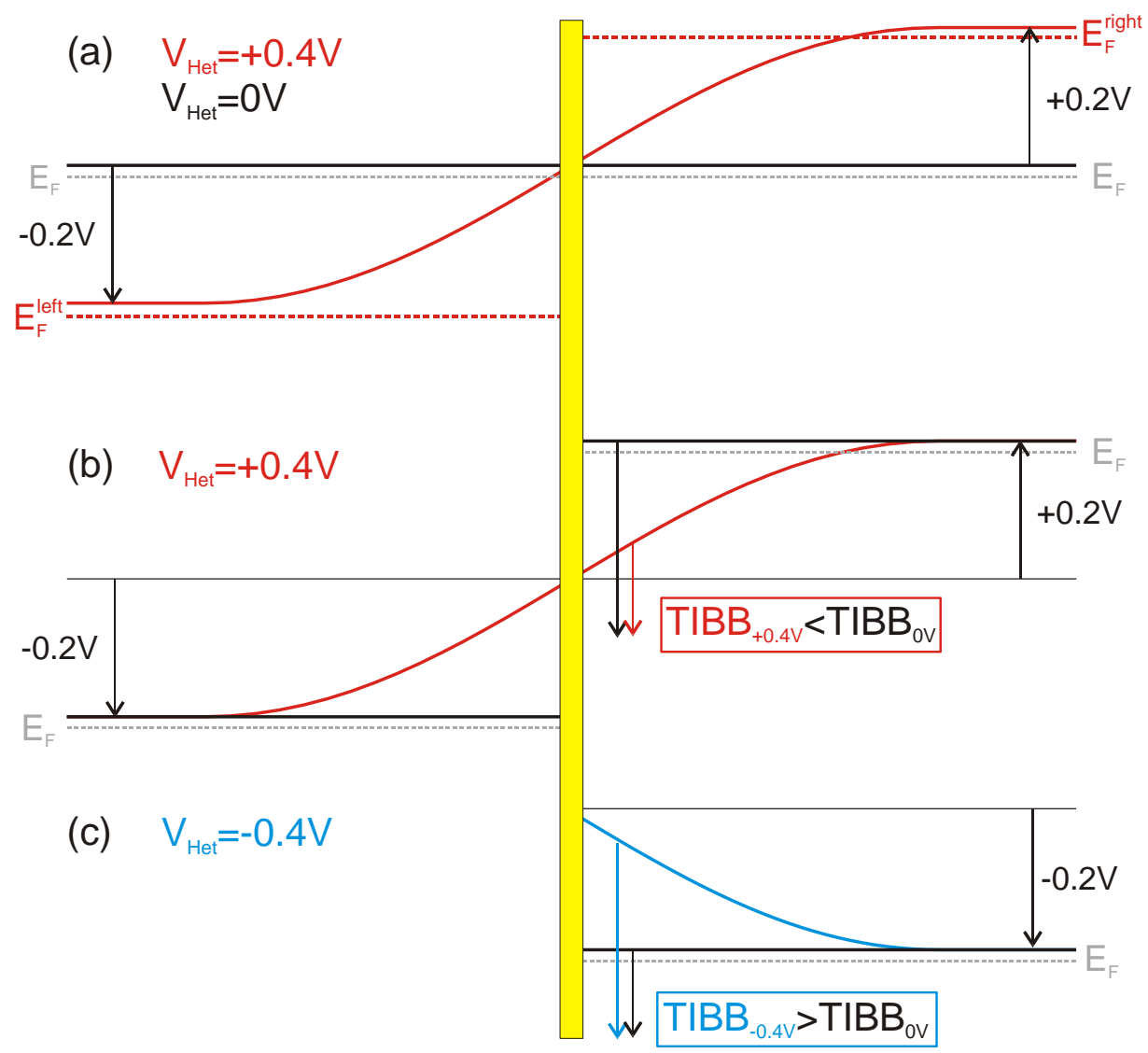

Figure 7.5: (a) Conduction band edge profile across the heterostructure for $V_{\mathrm{Het}}=0 \mathrm{~V}$ (black line) compared to $V_{\text {Het }}=+0.4 \mathrm{~V}$ (red line). (b) To compare the different amount of TIBB, the whole left band profile for $V_{\text {Het }}=0 \mathrm{~V}$ is shifted by $-0.2 \mathrm{~V}$ and the whole right band profile is shifted by $+0.2 \mathrm{~V}$. Close to the AIAs layer (yellow) the amount of TIBB which is necessary for creating a tip induced state is smaller for $V_{\mathrm{Het}}=+0.4 \mathrm{~V}$ than for $V_{\mathrm{Het}}=0 \mathrm{~V}$ (c) The same consideration is done for $V_{\mathrm{Het}}=-0.4 \mathrm{~V}$ (blue line). Here the amount of TIBB which is necessary for creating a tip induced state is larger for $V_{\mathrm{Het}}=-0.4 \mathrm{~V}$ than for $V_{\mathrm{Het}}=0 \mathrm{~V}$.

To compare the difference inside the voltage drop, this voltage shift is applied in the sketch of Figure 7.5(b) to the conduction band edge profile for no applied lateral voltage (black line). The following assumption is made: A tip induced state occurs if the conduction band is shifted a certain amount of energy below the Fermi energy. Thus, a certain amount of TIBB is needed to create the state, which could be linked to the measured voltage. For the right side shown in Figure 7.5(b) this would mean that the TIBB needed for $V_{\mathrm{Het}}=+0.4 \mathrm{~V}$ is smaller than the TIBB needed for $V_{\mathrm{Het}}=0 \mathrm{~V}$. It is visualized by the black and red arrow. The tip induced state should therefore occur at a smaller negative voltage.

For the reverse applied lateral voltage $V_{\mathrm{Het}}=-0.4 \mathrm{~V}$ the right side is shown in Figure 7.5(c). Here the amount of TIBB needed to create the tip induced state is larger for $V_{\mathrm{Het}}=-0.4 \mathrm{~V}$ than for $V_{\mathrm{Het}}=0 \mathrm{~V}$. It is visualized by the black and blue arrow. The tip induced state should therefore occur at a larger negative voltage. For the left side the whole assumption can be inverted. 


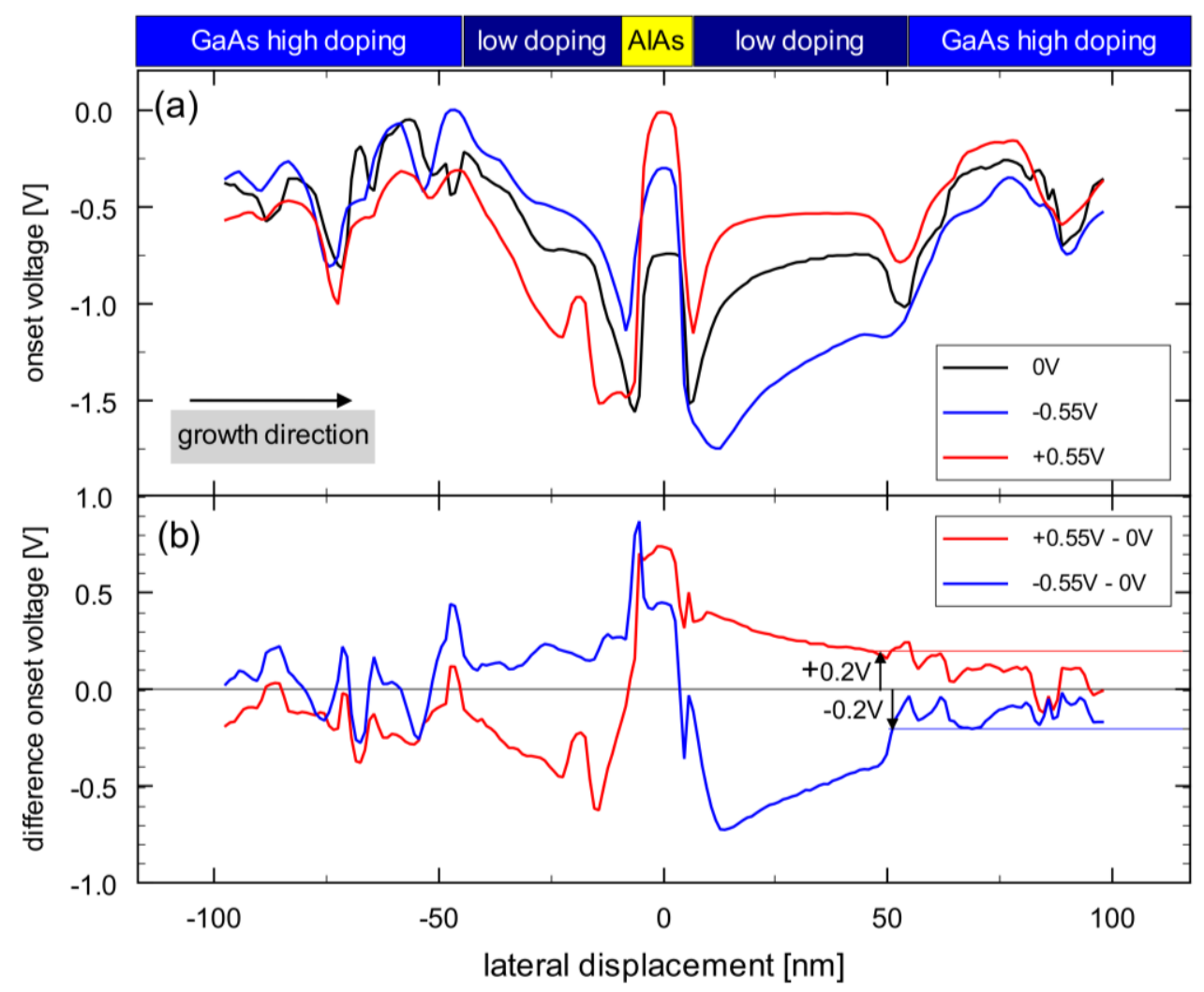

Figure 7.6: (a) The image shows the current onset voltage for negative voltage $V_{\mathrm{l}=-1 \mathrm{pA}}$, which gives the energetic position of the tip induced state. Different lateral voltage are applied 0V (black) $-0.55 \mathrm{~V}$ (blue) and $+0.55 \mathrm{~V}$ (red), the influence of the lateral voltage is clearly visible. In (b) the difference of the onset value is plotted. The onset voltage of $\mathrm{OV}$ is taken as a reference. The blue and the red horizontal lines on the right side are the zero voltage lines according to the consideration done in Figure 7.5.

This assumption is compared to the measurement. Figure 7.6(a) shows the extracted onset voltages for $V_{\mathrm{I}=1 \mathrm{p} A}$, which gives the energetic position of the tip induced state. (The corresponding differential conductivity sections are shown in Figure 6.6.) The proposed trend for the right side of the data can be seen in the low doping area of GaAs. For positive lateral voltage (red line) the tip induced state occurs at a smaller negative voltage and for the negative lateral voltage (blue line) the tip induced state occurs at a larger negative voltage. The inverted trend can be seen on the left side.

The tip induced state is energetically not constant in lateral direction. Therefore the profile of the tip induced state for $V_{\mathrm{Het}}=0 \mathrm{~V}$ is used as a reference. In Figure 7.6(b) the difference between the onset voltage of the tip induced state for $V_{\mathrm{Het}}=+0.55 \mathrm{~V}$ and $V_{\mathrm{Het}}=0 \mathrm{~V}$ is plotted by the red line, and the difference for $V_{\mathrm{Het}}=-0.55 \mathrm{~V}$ and $V_{\mathrm{Het}}=0 \mathrm{~V}$ is plotted by the blue line. (The shift of the tip induced state on high doped GaAs by $+0.2 \mathrm{~V}$ and $-0.2 \mathrm{~V}$, which is just due to the measurement technique, is indicated by the black arrows (compare Figure 7.5(a)).

The difference of the onset voltage gets larger for smaller distances to the AlAs layer. This is expected, as the difference of the onset voltage is linked to the difference of the potentials. According to the potential profile, the differences of the potentials get also larger with decreasing distance to the AlAs layer. 
The assumption in the beginning that a certain amount of TIBB is needed to create a tip induced state and that this amount of TIBB can be directly linked to the measured voltage is a very rough estimation. One has to consider that the screening is different for the different situations. For the situation of positive lateral voltage Figure 7.5(b, right side) even without the influence of the tip a $2 \mathrm{~d}$ electron gas parallel to the AlAs layer has already formed. Creating a tip induced state should be easier than for flat bands. For the situation of negative lateral voltage Figure 7.5(c, right side) a $2 \mathrm{~d}$ depletion layer is formed parallel to the AlAs layer and the potential of the tip has to compensate a positive potential. The TIBB as a function of voltage is different for positive band bending and negative band bending. Thus, the measured voltage for the flat band and the depletion layer are not directly comparable.

But still, the developing of the tip induced state is linked to the potential profile of the biased heterostructure. Together with the extraction of the potential at positive voltage (section 6.1), the evolution of the tip induced state could give useful information for further studies of the potential landscape in different biased heterostructures. 


\section{Summary}

The main part of this thesis was the investigation of effects caused by the space charge region in semiconductors using scanning tunneling microscopy and spectroscopy. The influence of a single donor and multi donor systems on the space charge region in homogeneous n-doped GaAs was studied. The importance of the space charge region for transport measurements was shown by the investigation of biased resonant tunneling diodes in the STM.

The first part of the thesis started with the investigation of single donor ionization. The charged tip induces a potential in the semiconductor. This potential is screened by the ionization of donors and a space charge region is build up. The ionization point of a single donor as a function of lateral tip donor distance and the applied tip-sample voltage was resolved. It was compared to the homogeneous simulation of the space charge region of the three dimensional tip-sample system and a good agreement was determined. By the study of single donors in different depth below the surface, it was found that the donor binding energy enhances the closer the donor is positioned to the surface. The enhancement causes an unexpected ionization sequence: A donor which is embedded a few layers below the surface is ionized at lower voltages than a donor which is embedded in a layer closer to the surface. The sequence of the ionization was verified by laterally resolved spectroscopy measurements.

The interaction of a two-donor-system was studied. It was shown that the influence of a second donor causes an ionization gap on a donor and vice versa. At a certain position of the tip it is possible to ionize two donors at the same applied voltage. At that position the system is bistable. Either one donor is ionized or the second donor is ionized. For a special donor configuration the system can be stable up to a few milliseconds, which was detected with the STM setup.

In the second part of this thesis a resonant tunneling diode was investigated. Whereas resonant tunneling diodes have been studied intensively by transport measurements, the investigation in the STM has not been intense so far. Thus, some features known very well from single $I(V)$-characteristics could be measured spatially resolved for the first time: 
Spectroscopic features occurring for positive tip-sample voltage on the AlAs barrier were described by tunneling due to electrons stemming from the X-point of AlAs. The existence of such a tunneling channel confirmed a model that at negative tip-sample voltage a resonant $\Gamma$-X-valley tunneling process is resolved. The resonant tunneling process is possible due to the modification of the potential profile by the tip. A lateral voltage applied to the heterostructure was used to modify the potential landscape. An impact of that voltage on the energetic position of the resonance was seen. The trend of the energetic shift is in accordance to the proposed model. The spatial position of the resonance is dependent on the cleavage surface either (011) or (0-11). The asymmetry due to the cleavage surface is similar to the observation of asymmetric topography contrast of acceptors in GaAs described in the literature.

The wave function of a quantum dot inside a resonant tunneling diode was mapped. Up to five energy levels could be resolved independently. The wave function was compared to the solution of the quantum mechanical one dimensional harmonic oscillator problem. A good agreement in terms of the energy, as well as on the spatial extension of the wave function was obtained. The energetic position of the lowest wave function inside an applied electric field was studied. An influence of the applied electric field was detectable and visualized. As an outlook further studies of the wave function inside an electric field could give insight in the Stark effect of the quantum dot state.

Up to now the study of semiconductor heterostructures with applied lateral voltage in combination with highly resolved STM and STS measurements has not been very intense. In this thesis the sample contacting in the STM setup allows applying a lateral voltage across the heterostructure in addition to the usual tip-sample voltage. Spectroscopy measurements with different lateral voltages and taken at the same position were used to extract the potential profile of the biased heterostructure. A simulation of the heterostructure gives the potential profile due to the lateral voltage as well as the position of the space charge region. By comparing the measurements with the simulation, it was proposed that the negative and positive charges are located almost in the same distance to the heterostructure.

The direct measurement of the potential profile of a heterostructure which has a doping gradient is not possible due to the effect of tip induced band bending in GaAs. Thus, laser light was irradiated on the sample to create electron hole pairs. The idea is that holes will accumulate at the surface and its positive charge is comparable to the donor concentration in the doped area, this will reduce the effect of tip induced band bending. For several spectroscopy measurements with different laser intensities taken at the same position the reduction of the tip induced band bending effect was shown. It was evaluated that for high laser intensities the spectroscopy measurement of the high doped and undoped areas are comparable. This enabled to extract the potential profile of the heterostructure. The measurement also showed that the radiated laser light created a photo induced current on the AlAs barrier. As an outlook, further studies of the heterostructure under laser radiation could lead to interesting results.

The tip induced state in low doped GaAs was investigated close to an AlAs barrier. In measurements up to now a tip induced state in GaAs was always disturbed by surrounding donors. In this thesis a sample with an undoped GaAs layer was used, which enables to investigate this state without the perturbation of the potential of donors. Furthermore an AlAs was embedded inside the undoped layer to modify the energetic position of the tip induced state. The energetic shift of this state as a function 
of distance to the AlAs barrier could be measured. This shift was compared to a simulation of the potential landscape of the tip-sample system. The trend of the measurement could be verified by the simulation. By applying an external lateral voltage, the energetic position of the state was modified. The influence of the external lateral voltage on the state gave insight into the space charge which is build up in the heterostructure. 



\section{A Appendix}

\section{A.1 Flat band condition}

\section{A.1.1 Determining the flat band condition from barrier height measurement}

If the tip and the sample are brought together a contact potential will form, as the tip and the sample are different materials. Due to the small screening inside the semiconductor the bands are shifted. The amount of the shifting is dependent on the applied voltage. The voltage where the bands in the semiconductor are flat is not known initially. A very simple approach is to use the electron affinity of the sample $\chi_{\text {s }}$ and the work function of the tip $\phi_{t}$ known from literature to evaluate the voltage of the flat band condition $V_{f b}$ with the relation, which is similar to the concept of the Schottky contact [14]:

$$
\chi_{s}-\phi_{t}=V_{f b}
$$

The work function of polycrystalline tungsten $\phi_{t}=4.5 \mathrm{eV}$ [98] and electron affinity of GaAs $\chi_{s}=4.1 \mathrm{eV}[25]$ are known for the ideal clean surface from literature. The sample surface does fulfill those requirements of an ideal crystalline surface and its electron affinity can be taken from the literature value. In contrast, the tip is not a perfect flat plane, but rather a disordered accumulation of atoms, with a round geometry. This can influence the work function considerably. It has been shown by Besocke and Wagner [48] that a changed morphology of the surface decreases the work function for tungsten by up to $0.6 \mathrm{eV}$. As the change of the work function for the special tip geometry is not known, it has to be evaluated by the measurements. One technique to get a value for the tips work function is to measure the apparent barrier height for different voltages. 
(a)

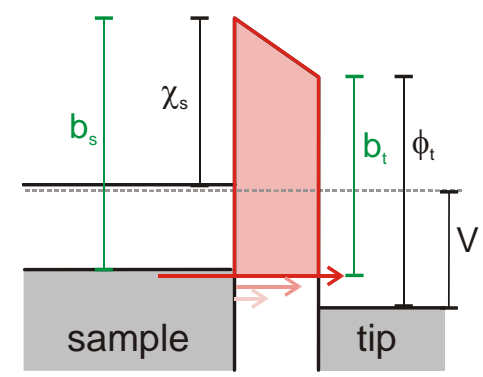

(b)

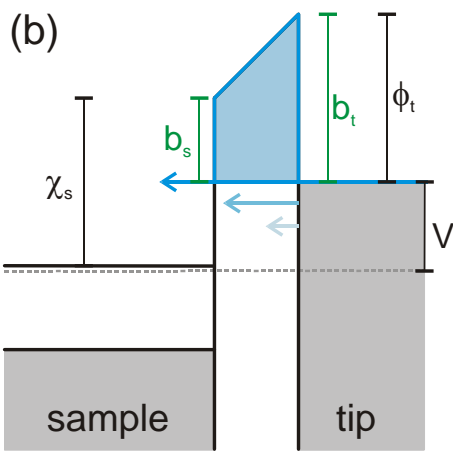

Figure A.1: Sketch of the expected apparent barrier for (a) negative and (b) positive voltage. The magnitude of the apparent barrier height for both voltages is different. The apparent barrier height does not represent simply the mean value of the work function of the tip $\phi_{\mathrm{t}}$ and the electron affinity of the sample $\chi_{s}$ as the voltage has to be considered. The expected apparent barrier is the mean value of the barrier $b_{s}$ for the sample and $b_{t}$ for the tip. (For simplification the figure omits the TIBB.)

In Figure A.1 the expected barrier heights for negative and positive voltages are sketched as the colored areas (red and blue). As a first approximation the whole barrier is assumed to be a trapezoidal barrier. (In subsection A.1.3 the limitation of this approximation will be discussed). The main tunnel current is expected for electrons with the smallest barrier, as the tunneling probability is smaller for electrons with larger barrier heights; this is visualized by the stronger colored arrows in the figure. As a first description, only electrons with the smallest barrier are considered, deviations from this description will be discussed later in this paragraph. In Figure A.1 the work function is labeled with $b_{s}$ for the sample side and $b_{t}$ for the tip side. For negative voltage (Figure A.1(a)) the band gap $E_{g}$ has to be included, as well as the applied voltage. For positive voltage (Figure A.1(b)) one has to consider the applied voltage, which has to be subtracted from the electron affinity. For simplification the trapezoidal barrier is approximated by a rectangular barrier with the mean value of the tip barrier $b_{t}$ and the sample barrier $b_{s}$. In [99] it was simulated that this approximation is nearly correct. Then the apparent barrier height $\phi_{a b h}(V)$ is given by the following relation:

$$
\phi_{a b h}=\frac{b_{s}+b_{t}}{2}
$$

Including the voltage dependent $\operatorname{TIBB}(V)$, for positive voltage the relations for the tip and sample barrier $b_{t}$ and $b_{s}$ are:

$$
\begin{aligned}
& b_{t}=\phi_{t} \\
& b_{s}=\chi_{s}-e V+\operatorname{TIBB}(V)
\end{aligned}
$$




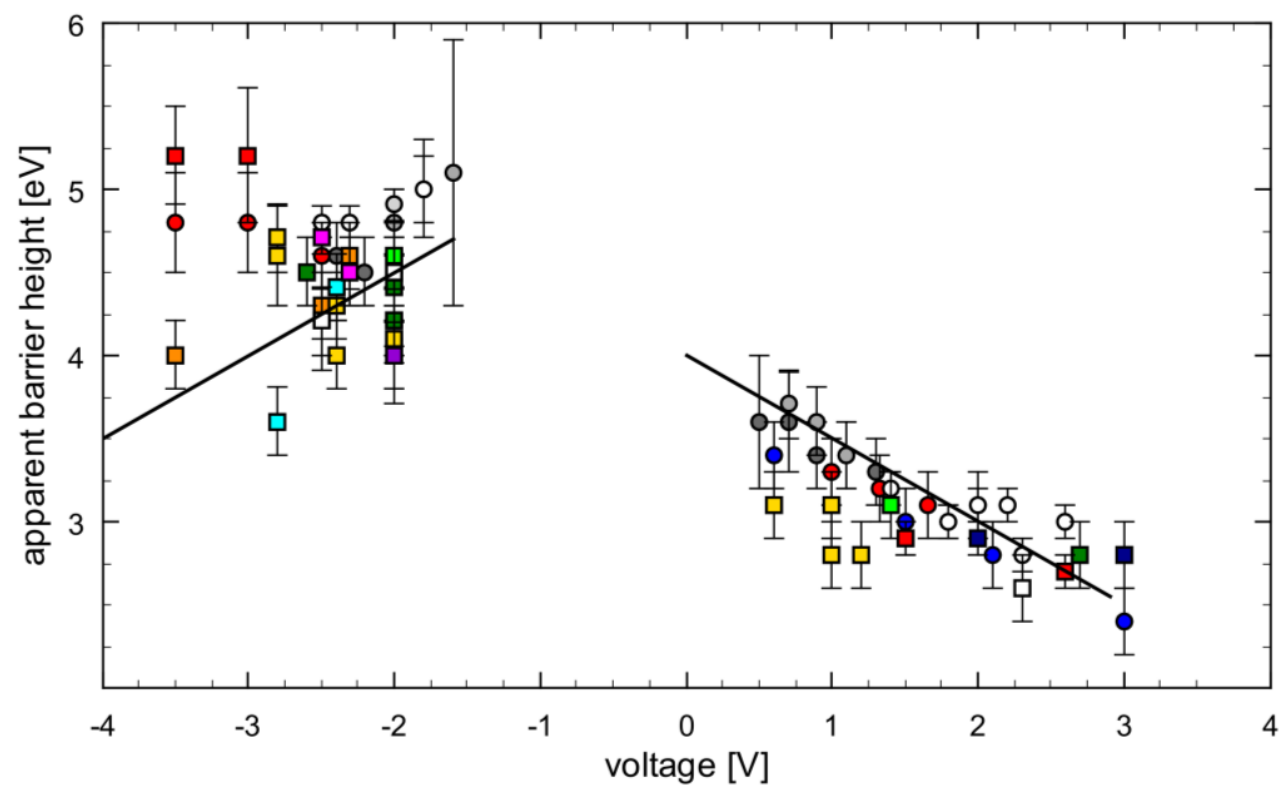

Figure A.2: Apparent barrier height as a function of voltage, for different positions and tips (indicated by different colors). The circles are obtained from multibias topography measurements, the squares are obtained from spectroscopy measurements. As a guide to the eye the expected trend of the work function (excluding the effect of TIBB) is shown by the black line. For large negative voltage a derivation of that trend can be seen.

For negative voltage the relations for the tip and sample barrier are:

$$
\begin{aligned}
& b_{t}=\phi_{t}+E_{g}+e V-\operatorname{TIBB}(V) \\
& b_{s}=\chi_{s}+E_{g}
\end{aligned}
$$

From the measured apparent barrier height at different voltages, the work function of the tip can now be evaluated. The evaluated work function is used to determine the flat band condition for the tip-sample geometry using equation (A.1).

In Figure A. 2 measurements of the apparent barrier height are shown as a function of the applied voltage. The circles are extracted from multibias topographies at different current setpoints and the squares are taken from spectroscopy measurements. The black line shows, how the expected apparent barrier should develop according to equation (A.3) and (A.4) using a tip work function of $4 \mathrm{eV}$. The $\operatorname{TIBB}(V)$ is not included into the black line, as it should make a minor correction. This was discussed in more detail in [99]

The measured apparent barrier height follows the expected apparent barrier height quite well, However for large negative voltages $(V>-3 \mathrm{~V})$ a deviation from the expected value can be seen, as the measured barrier height is larger than the expectation. 
(a)

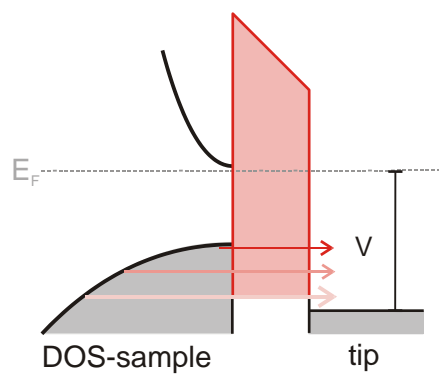

(b)

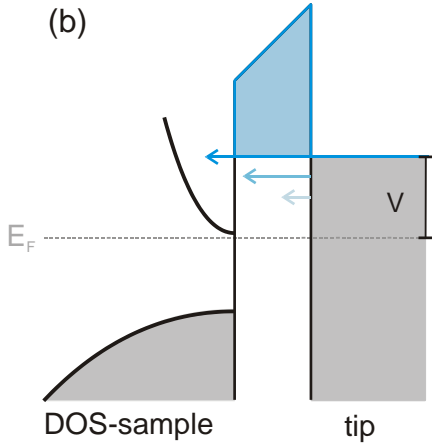

Figure A.3: (a) For large negative voltages the DOS of the valence band increases for smaller energies. Electrons close to the valence band maximum have a larger tunneling probability (indicated with the stronger color), but less electrons are available for tunneling due to a smaller DOS (indicated with a larger arrow). The total apparent barrier height is a sum of all tunneling electrons. (b) The situation is shown for positive voltage

Up to now, only electrons with the smallest barrier height have been considered. Because their tunneling probability is largest these electrons should dominate the tunnel current. In a next step all tunneling electrons, as well as the density of states (DOS) should be included. The DOS will be included due to the effective mass approximation. Considering the situation for negative voltages (Figure A.3(a)), electrons energetically deep in the valence band have a larger DOS than electrons close to the valence band maximum, thus more electrons are available for tunneling. This compensates a larger barrier, which means a smaller tunneling probability for electrons deep in the valence band. Electrons close to the valence band maximum have a higher tunneling probability due to a smaller barrier, but the amount of available electrons is small as the DOS is small. Summing up, one would expect a larger barrier height, than in the simple model. For positive voltage the electron with the highest energy does also have the largest DOS, which is illustrated in Figure A.3(b). Also here one would expect a larger apparent barrier height, but the effect should not be so strong like for negative voltage.

The total apparent barrier height which is measured in STM is composed of the barriers of all tunneling electrons. For a better understanding of the measured data a more detailed simulation was done, than just the simple model. The apparent barrier height of each electron-energy was weighted with the corresponding densities of states DOS and the corresponding tunneling probability $\mathrm{T}$ for an electron. The tunneling probability of the trapezoidal barrier was evaluated by using the WKB approximation. This is basically illustrated in Figure A.3. For a specific voltage, this weighted apparent barrier height was summed up over the energy range where electrons are available for tunneling. For a better readability the detailed equations are removed into subsection A.1.5. 


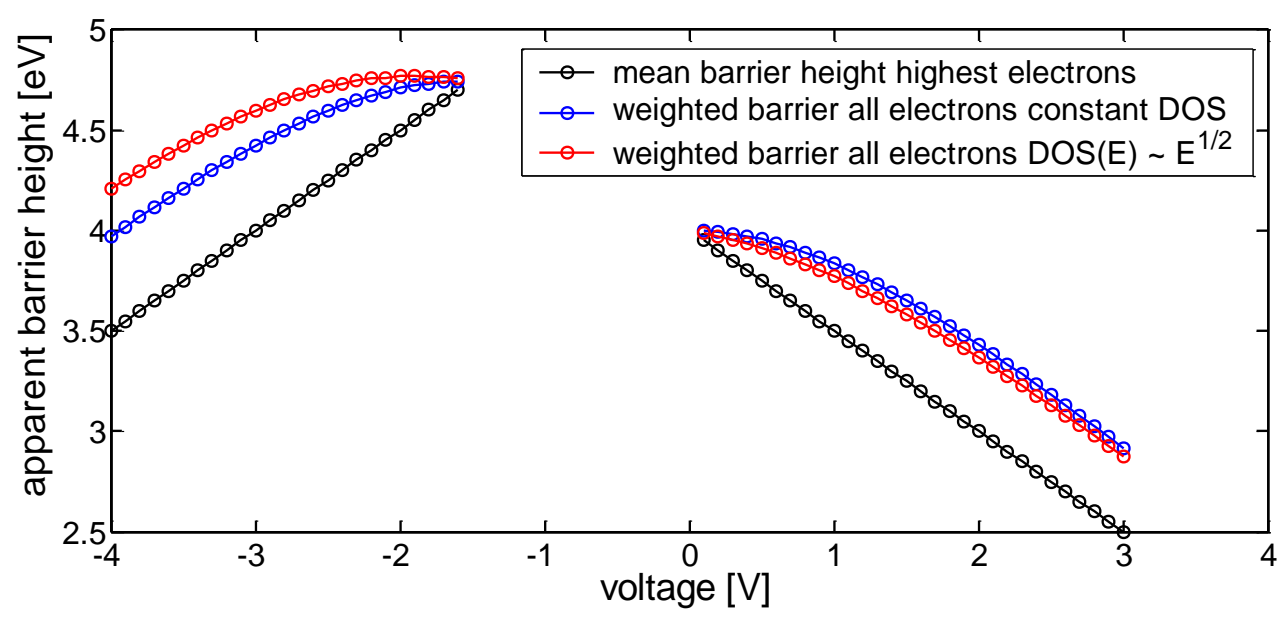

Figure A.4: Simulation of the voltage dependence of the apparent barrier height. The black line shows the apparent barrier height obtained by the simple model of using only the electrons with the highest barrier. The blue line shows the apparent barrier height, including all electrons with their transmission probability and a constant DOS. For the red line the DOS was included using the effective mass approximation.

Figure A.4 compares the simulation of the apparent barrier height with the simple model, which included only electrons with the smallest barrier (black line) with the simulation where all tunneling electrons are considered. In that simulation the electrons are weighted with their tunneling probability and a constant DOS (blue line) and a $\operatorname{DOS}(E) \sim E^{1 / 2}$ (red line). Including all electrons and a constant DOS leads to an enlargement of the apparent barrier height, including all electrons with the $\operatorname{DOS}(E) \sim E^{1 / 2}$ enlarges the apparent barrier height for negative voltage, but decreases the barrier for positive voltage compared to the constant DOS, which was in principle expected.

Figure A.5 compares the measured data with the simulation using different tip work functions. From the plot one can see that a tip work function of $4.5 \mathrm{eV}$ is too large to explain the data points. For positive voltage a work function of $3.5 \mathrm{eV}$ is a good value, for negative voltage the value of $4.0 \mathrm{eV}$ is a good value. Using equation (A.1), the flat band condition of the sample would be expected to be from 0.1 to $0.6 \mathrm{~V}$. 


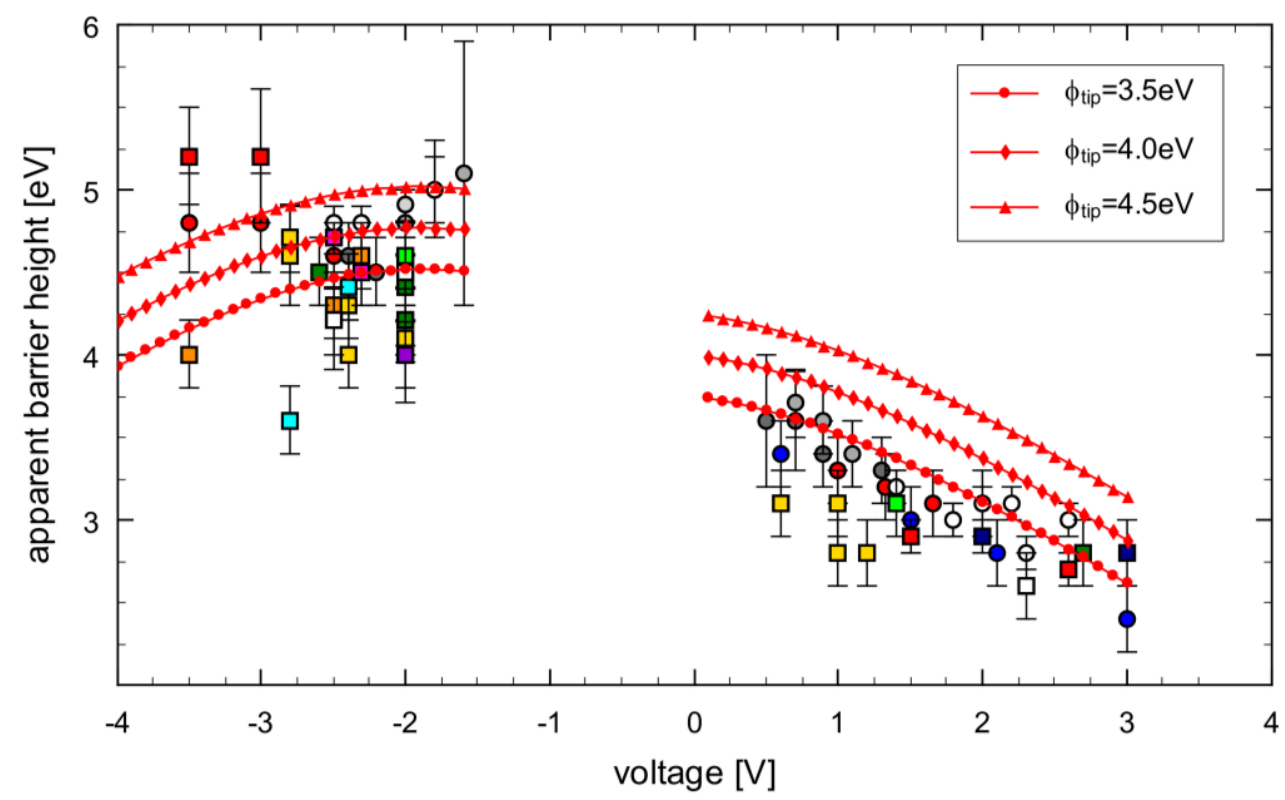

Figure A.5: Comparison between the measurement and the expected voltage dependent apparent barrier height for different tip work functions. The tip work function of $4.5 \mathrm{eV}$ is too large for the data. For positive voltage the work function of $3.5 \mathrm{eV}$ is a good value, for negative voltage the work function of $4.0 \mathrm{eV}$ is a good value.

\section{A.1.2 Determination of the flat band condition from spectroscopy}

In the last subsection the position of the flat band condition was only evaluated by the measurement of the apparent barrier height. In chapter A.1.3 it will be discussed that this measurement technique has some limitations. Thus, it is important to cross check the obtained flat band conditions with the spectroscopic data. Figure A.6 shows two differential conductivity spectra taken at different positions and at different current setpoints of $-2 \mathrm{~V}, 0.5 \mathrm{nA}$ and $+0.6 \mathrm{~V}, 0.1 \mathrm{nA}$. The spectra are an average over 100 spectra which are obtained on the free surface ${ }^{8}$. In both spectra one can see that the current for positive voltage starts at a voltage smaller than $\sim 20 \mathrm{mV}$. This is an indication that the tip induce band bending is very small positive $(<20 \mathrm{meV})$ or even negative, as the applied voltage has to be larger than the TIBB to obtain a tunnel current. In the expected band gap (grey shaded area) one can identify peaks of differential conductivity (three for the blue line, one for the red line). These peaks can be identified as tip induced states (TIS), which arise due to negative TIBB, when the tip forms a tip induced quantum dot in the semiconductor [18]. The first well visible TIS occurs at a voltage of $\sim-0.5 \mathrm{~V}$ (blue line), which means that the TIBB has to be negative at that voltage. For the red line the first TIS occur at $\sim-1.2 \mathrm{~V}$, this can be due to the different setpoints of the spectroscopy, which leads to different tip-sample distances. As the tip of the red line is more far away from the surface than the tip of the blue line, the current obtained from the TIS could be smaller than the noise of the IV-converter. At a voltage of $\sim-1.5 \mathrm{~V}$ one can identify the onset of the valence band.

\footnotetext{
${ }^{8}$ The free surface should mean as far away as possible from any visible donor.
} 


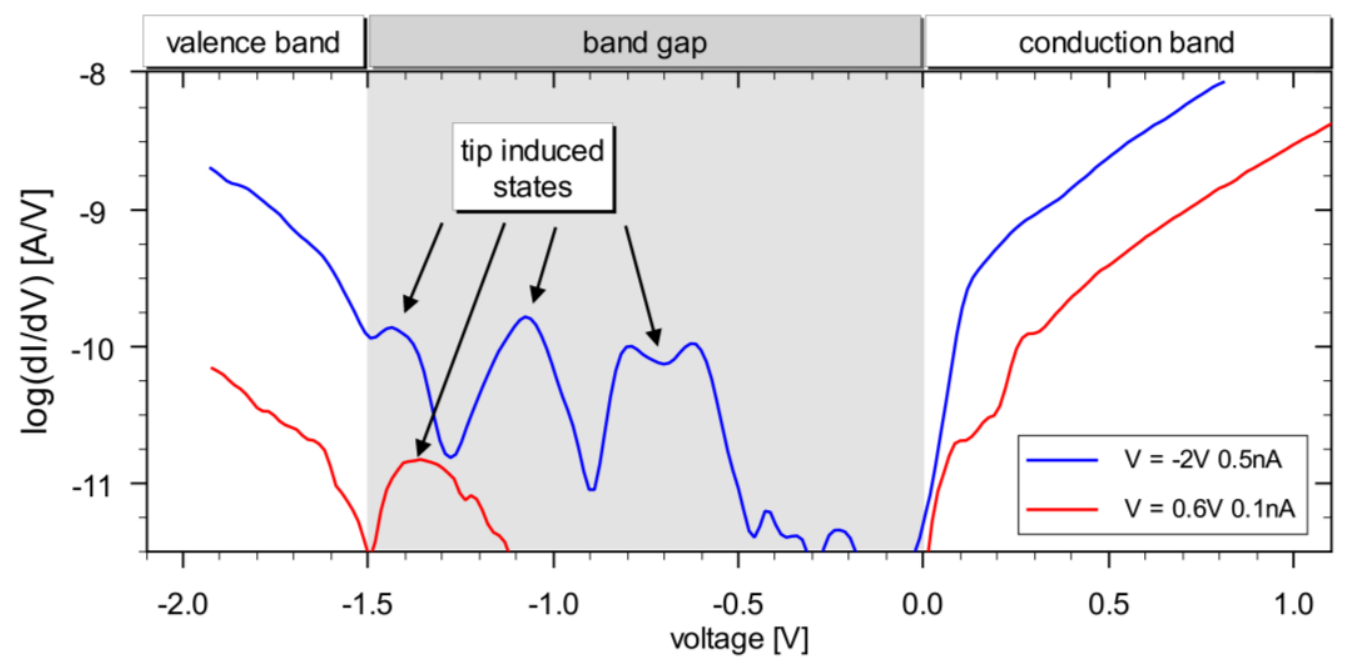

Figure A.6: Differential conductivity spectra of two data sets. The blue line shows a spectroscopy at a setpoint of $-2 \mathrm{~V}$ and $0.5 \mathrm{nA}$ the red line shows a spectroscopy at a setpoint of $+0.6 \mathrm{~V}$ and $0.1 \mathrm{nA}$. Within the expected band gap (grey shaded area) peaks in the differential conductivity occur, these peaks can be identified as tip induced states. For positive voltages the current is directly starting at $0 \mathrm{~V}$

The interpretation can be verified by using the lateral information of the spectroscopy. In Figure A.7 a laterally resolved spectroscopy is shown. Laterally resolved $\mathrm{d} I / \mathrm{d} V(x, y)$-maps are shown at a voltage of (b) $+0.4 \mathrm{~V}$, (c) $-0.7 \mathrm{~V}$ and (d) $-1.6 \mathrm{~V}$, respectively. For positive voltage an ionization ring of a donor deep below the surface is visible, the ring starts at the same voltage, where the current for positive voltage starts $\sim 20 \mathrm{mV}$. The existence of such an ionization ring is an indication that the TIBB is positive at that voltage. The origin of the ionization ring is discussed in detail in chapter 3. For negative voltage of $-0.7 \mathrm{~V}$ the tip induced states are visible. Due to the spatially varying potential landscape, also the voltage position of the TIS changes, which leads to local varying states at a certain voltage. At larger negative voltage $-1.6 \mathrm{~V}$ the onset of the valence band has started, which can be seen due to the higher $\mathrm{d} I / \mathrm{d} V$ signal and that the signal does not vary spatially that much like in Figure A.7(d) any more. 

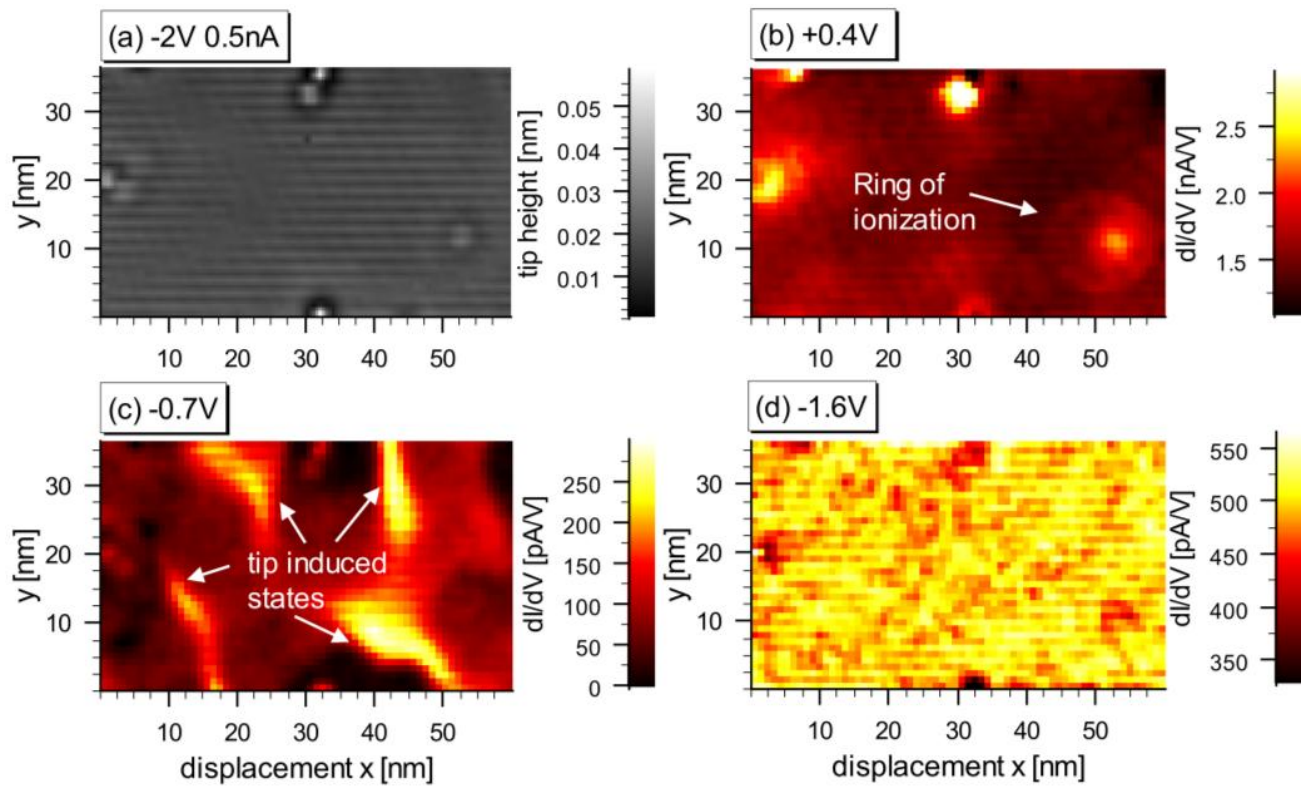

Figure A.7: (a) Constant current topography image taken at a setpoint of $-2 \mathrm{~V}$ and $0.5 \mathrm{nA}$. (b-d) laterally resolved $\mathrm{d} / \mathrm{d} V(x, y)$-maps at a voltage of $+0.4 \mathrm{~V},-0.7 \mathrm{~V}$ and $-1.6 \mathrm{~V}$, respectively. (b) For positive voltage $+0.4 \mathrm{~V}$ ionization rings of the donors can be seen, this is an indication of positive TIBB. (c) In the expected band gap at $-0.7 \mathrm{~V}$ tip induced states can be identified; (d) shows the onset of the valence band.

Summarizing the spectroscopic features one obtains that the flat band condition should occur in a range of $-0.5 \mathrm{~V}$ to $0.02 \mathrm{~V}$. Using the result of section A. 1 and the current section, a flat band condition of $\sim 0 \mathrm{~V}$ is reasonable, which would mean having a tip work function of $4 \mathrm{eV}$.

\section{A.1.3 Limitation of the apparent barrier height measurement}

The real potential seen by the tunneling electron cannot be simply described by a trapezoidal barrier in which the barrier changes in space abrupt to a certain value. In reality it will change smoothly in space to that value. This is cause by a dipole layer at the surface, which is formed by electrons leaking into the vacuum barrier. For a theoretical treatment of the surface potential this dipole, as well as exchange and correlation effects have to be included, which was done by Baredeen as well as by Lang and Kohn [100, 101]. From these results it is known that one can treat the electron outside the crystal as individually moving in a classical image potential [12]. Compared to the classic image potential the trapezoidal barrier would be too large.

Teague published an investigation of tunneling between two gold electrodes, where he calculated the tunnel current distance dependent. The tunnel current was calculated for a classical image potential and by using the WKB approximation. In Figure A.8 his obtained tunnel current values were taken and converted into the apparent barrier height. Figure A.8 shows that the obtained apparent barrier height is almost the value of the assumed work function, which corresponds to the description like the trapezoidal barrier. This observation is due to the distance dependent reduction of the image potential barrier, which is explained by Chen [12]. 


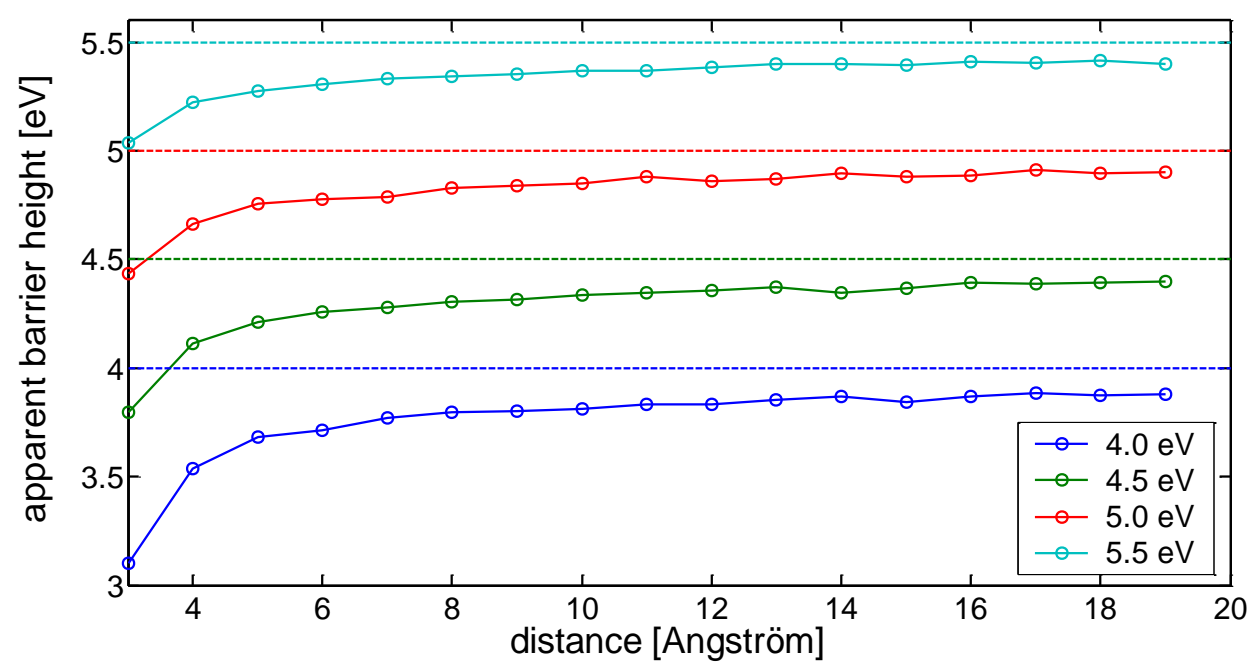

Figure A.8: Tunnel current taken from Teague [103] and converted into an apparent barrier height.

However the discussion of the voltage where the bands are flat is still present. In 2010 Wijnheijmer et al. [102] published a paper, where the difficulty of the apparent barrier height measurement is investigated with a simulation according to Chen [12]. In the simulation the authors uses the classical image potential and calculating the apparent barrier height with the transfer matrix algorithm. Their result is that the measured apparent barrier height is about $1 \mathrm{eV}$ smaller than the work function. Their main argument is that according to their used simulation values one should see an enhancement of the apparent barrier height with decreasing distance to the surface. They see it for p-doped material. As we do not see an enhancement of the apparent barrier height in our measurements of n-doped material, we conclude that the Flat band conditions, which we obtain from the simple trapezoidal treatment, are correct.

\section{A.1.4 Laterally resolved apparent barrier height}

The apparent barrier height measured could also be disturbed by effects which occur due to the tip-sample distance. As an example a laterally resolved and voltage dependent apparent barrier height measurement $\phi(x, V)$ across the heterostructure is shown in Figure A.9(a). The apparent barrier height was extracted from two $I(x, V)$ spectroscopies taken at the same lateral x-position and at two different current setpoints, which means two different tip-sample distances. One of the spectroscopies is shown as a differential conductivity section $\mathrm{d} I / \mathrm{d} V(x, V)$ in Figure A.9(b).

For positive $>1 \mathrm{~V}$ and large negative $>-2 \mathrm{~V}$ voltage the apparent barrier height stays almost constant. In this voltage range the current is large, and dominated by the valence or conduction band. In the voltage range of the band gap $0 \mathrm{~V}$ to $-1.5 \mathrm{~V}$ the apparent barrier height has increased by up to a difference of $2 \mathrm{eV}$. For that voltage the current is small and not stemming from the valence or conduction band directly. Instead the tunneling channel is the tip induced state. This state is created by forming a quantum dot due to the potential of the tip. The size of the tip induced quantum dot is very sensitive on the tip-sample distance and will occur at different voltages for different tip-sample distances. This means that for a specific voltage, the current 

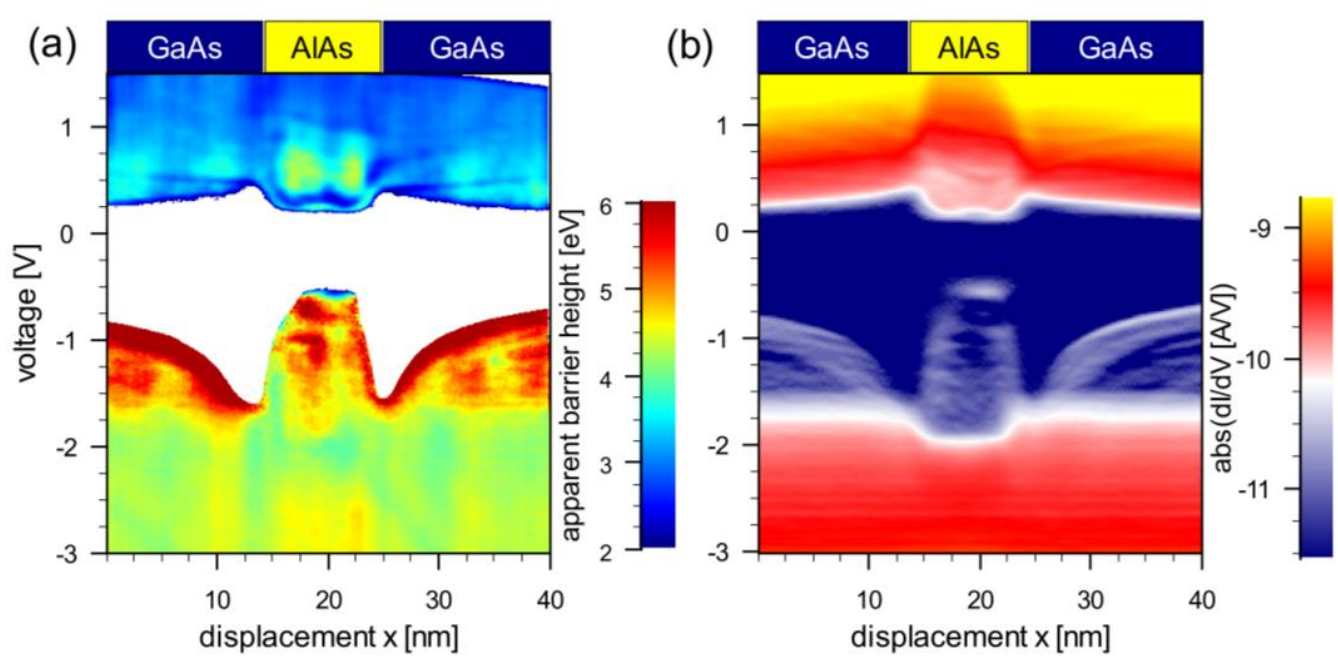

Figure A.9: (a) Apparent barrier height voltage dependent and laterally resolved. (b) As a comparison the spectroscopy is shown.

occurs at one tip-sample distance but is vanished at the second tip-sample distance. The apparent barrier height is determined by comparing the current at the same voltage at different tip-sample distances. The above mentioned effect will cause a large difference in the measured apparent barrier height.

\section{A.1.5 Calculation of the Barrier height}

In the following paragraph the equations, which were used for the simulation in subsection A.1.1 should be shortly summarized. The total measured apparent barrier height $\phi_{a b h}{ }^{\text {weighted }}$, is the sum of the apparent barrier height $\phi_{a b h}$ of each electron, which is available for tunneling at a specific voltage $V$. The apparent barrier height $\phi_{a b h}\left(E_{e l}\right)$ of each electron-energy $E_{e l}$ was weighted with the corresponding densities of the states $\operatorname{DOS}\left(E_{e l}\right)$ and the corresponding tunneling probability $\mathrm{T}\left(E_{e l}\right)$ for an electron:

$$
\phi_{a b h}^{w e i g h t e d}=\frac{\sum_{E_{e l}=0}^{V} \mathrm{~T}\left(E_{e l}\right) \cdot \operatorname{DOS}\left(E_{e l}\right) \cdot \phi_{a b h}\left(E_{e l}\right)}{\sum_{E_{e l}=0}^{V} \mathrm{~T}\left(E_{e l}\right) \cdot \operatorname{DOS}\left(E_{e l}\right)}
$$

Figure A.10 visualizes the equation for (a) negative voltage and (b) positive voltage. The normalization is done by the denominator. The summation is done over the energy range where electrons are available for tunneling. The electron with the highest energy is labeled to have the "electron energy" $E_{e l}=0$. (Note that this is not meant to be the electron energy in the tip or the sample). For positive voltage the range of the sum is: $0<E_{e l}<V$, for negative voltage the band gap $E_{g a p}$ has to be considered and the range of the sum has to be interchanged by: $0<E_{e l}<-V-E_{g a p}$. The density of states for parabolic bands is included within the effective mass approximation. 
(a)

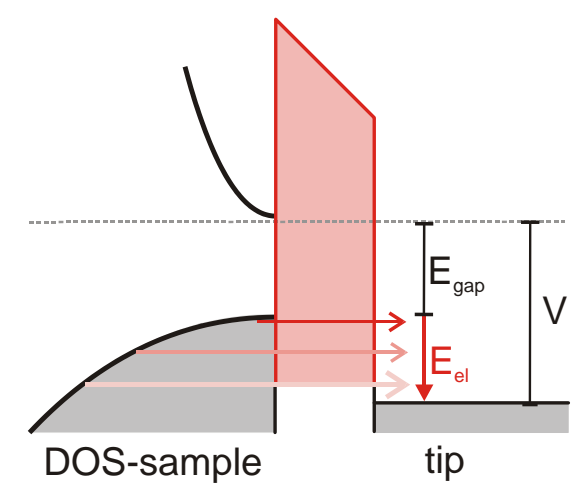

(b)

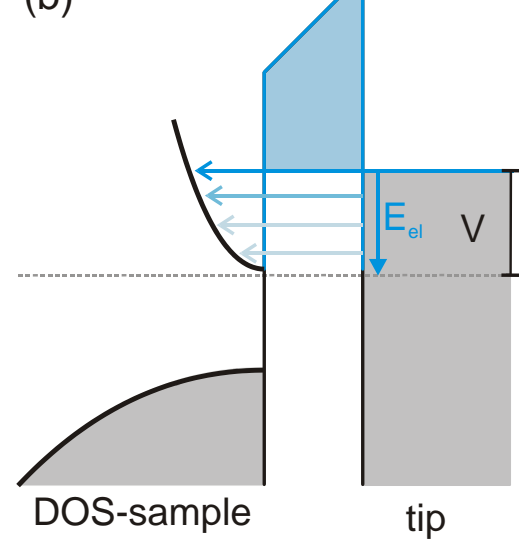

Figure A.10: Visualizing of the equation (A.5) for (a) negative voltage and (b) positive voltage. The mean apparent barrier height of an electron is multiplied with the corresponding transmission probability and the corresponding density of states. For a fixed voltage the obtained value for each electron is summed up. The electron with the smallest barrier is set to have an "electron-energy" $E_{e l}=0$. The band gap $E_{\text {gap }}$ has to be considered for negative voltage.

The tunneling probability $\mathrm{T}\left(E_{e l}\right)$ for an electron was evaluated using the WKBapproximation by Wentzel, Kramers and Brillouin. The equation can be found in every standard quantum mechanics book (see for example [104]). The potential used in the WKB-integral was the trapezoidal barrier, with a tip-sample distance $d$, expressed by the following equation:

$$
V(x)=b_{s}\left(E_{e l}\right)+\frac{b_{t}\left(E_{e l}\right)-b_{s}\left(E_{e l}\right)}{d} x \quad \text { for } \quad 0<x<d
$$

The tip barrier $b_{t}$ and sample barriers $b_{s}$ are enlarged by the "electron-energy" $E_{e l}$ :

$$
b_{t}\left(E_{e l}\right)=b_{t}(0)+E_{e l} \text { and } b_{s}\left(E_{e l}\right)=b_{s}(0)+E_{e l}
$$

The apparent barrier height for an electron having an additional electron-energy is included by the mean value into the equation (A.5):

$$
\phi_{a b h}\left(E_{e l}\right)=\frac{b_{t}\left(E_{e l}\right)+b_{s}\left(E_{e l}\right)}{2}
$$




\section{A.2 Simulation of the heterostructure}

The band edge profile of the heterostructure and the influence of the surface and the tip are simulated, to confirm the interpretation in this thesis.

As a first step the electrostatic problem of the heterostructure without InAs, tip and surface is considered. The Poisson-equation has to be solved self consistent by including the charge distribution for the different doping and materials. For the simulation the commercial program COMSOL Multiphysics was used, which solves the Poisson-equation self consistent based on the finite element method. The advantage of this finite element method is that also complex three dimensional structures can be solved numerically, which will be relevant if one wants to study the electrostatic problem of the heterostructure including the tip and the surface. A detailed description how to combine the simulation program to the special STM geometry can be found in [90]. The physically relevant functions which have to be included to the program package will be summarized shortly in the following paragraph.

The charge density $\rho$ in the semiconductor as a function of the chemical potential $\mu_{\mathrm{c}}$ has to be included in the simulation for the different doping concentration of GaAs as well as for the different materials GaAs and AlAs. The equations which describe the charge density in the semiconductor are summarized in the following paragraphs (compare [105] or [14]). The conduction band at the $\Gamma$-point and the $X$-point ${ }^{9}$ is considered for the negative charge. The positive charge is considered by the twofold valence band with heavy and light holes and the doping concentration of the n-doped semiconductor.

The DOS for parabolic bands within the effective mass approximation are:

$$
D O S(E)=\left(2 m^{*}\right)^{3 / 2} \frac{1}{2 \pi^{2} \hbar^{3}} \sqrt{E-E_{0}}
$$

In this equation $m^{*}$ are the corresponding density of states effective masses, the used values are summarized in Table $3 . E_{0}$ is the position of the corresponding band edge. Note that this equation is valid for the conduction bands, for the valence bands the quantities $E$ and $E_{0}$ change their sign. The GaAs conduction band edge minimum is defined to be zero energy.

Together with the Fermi-Dirac distribution $f$, the number of charge carriers can be calculated.

$$
f\left(E, \mu_{c}\right)=\frac{1}{1+\exp \left(\frac{E-\mu_{c}}{k_{B} T}\right)}
$$

The experiment was performed at $T=5 \mathrm{~K}, k_{B}$ is the Boltzmann constant and $\mu_{\mathrm{c}}$ is the position of the chemical potential in the semiconductor. The following equation describes the distribution of the donors, the number 2 before the exponential term is due to the spin degeneracy.

\footnotetext{
${ }^{9}$ For GaAs the $\Gamma$-point is more relevant, for AlAs the X-point is more relevant.
} 

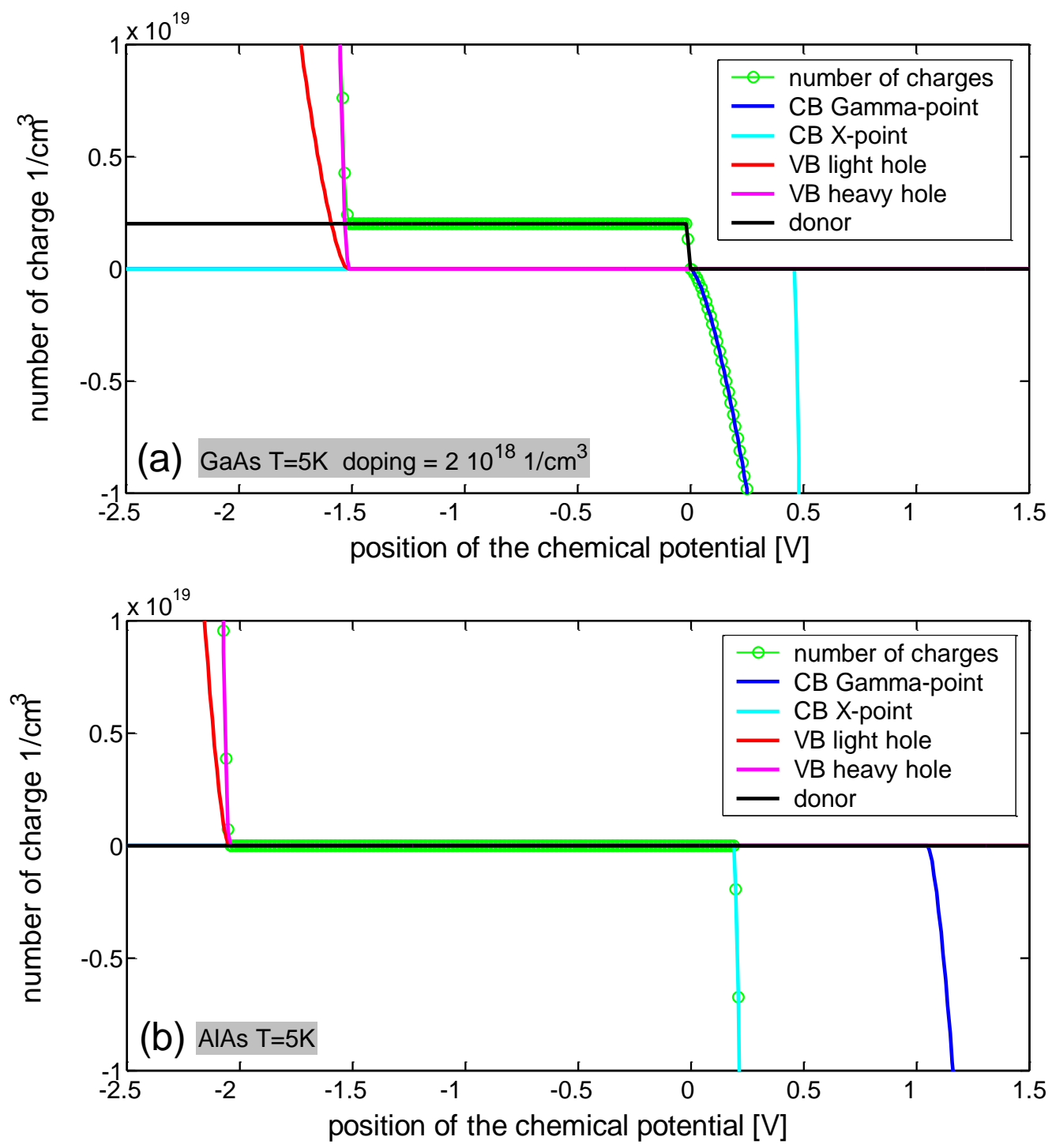

Figure A.11: The total number of charge density as a function of the position of the chemical potential is shown in green for the temperature of $5 \mathrm{~K}$. Zero potential is chosen to be the GaAs conduction band minimum. The contributions to the number of charges of the different bands are plotted in different colors. (a) GaAs with a doping concentration of $2 \cdot 10^{18} \mathrm{~cm}^{-3}$ is shown (b) AlAs is shown.

$$
f^{D}\left(E, \mu_{c}\right)=\frac{1}{1+2 \exp \left(\frac{E-\mu_{c}}{k_{B} T}\right)}
$$

The number of negative charge $n$, and the number of positive charge $p$, as well as the number of ionized donors $N_{D}$ are given by the following expressions: 


$$
\begin{gathered}
n\left(\mu_{c}\right)=\int_{E_{0}}^{\infty} f\left(E, \mu_{c}\right) \cdot \operatorname{DOS}(E) d E \\
p\left(\mu_{c}\right)=\int_{-\infty}^{E_{0}}\left[1-f\left(E, \mu_{c}\right)\right] \cdot \operatorname{DOS}(E) d E \\
N_{D}\left(\mu_{c}\right)=\int_{-\infty}^{\infty} f^{D}\left(E, \mu_{c}\right) \cdot \delta\left(E_{D}\right) \cdot N_{D} d E
\end{gathered}
$$

The energy of the donors was simply introduced by the donor binding energy $E_{D}=6 \mathrm{meV}$ which can be obtained for shallow donors by the hydrogen model. Note that the reduction of the donor energy and the broadening of the impurity band for highly doped semiconductors (due to the Mott transition) were not included in the simulation as the error by neglecting was estimated to be small.

In this thesis the integrals of equation (A.12)-(A.14) were derived numerically. The width of the step size $\mathrm{d} E$ was chosen to be $0.01 \mathrm{eV}$. The position of the chemical potential was varied from $-2.5 \mathrm{~V}$ to $1.5 \mathrm{~V}$ with a step size of $0.01 \mathrm{~V}$. The whole charge distribution as a function of the chemical potential $\rho\left(\mu_{\mathrm{c}}\right)$ consists of the number of holes in the heavy hole band $p^{h h}$ and in the light hole band $p^{\text {th }}$, the number of ionized donors $N_{D}$ the number of electrons in the $\Gamma$-valley $n^{\Gamma}$ and in the X-valley $n^{\mathrm{X}}$ :

$$
\rho\left(\mu_{c}\right)=e\left[p^{h h}\left(\mu_{c}\right)+p^{l h}\left(\mu_{c}\right)+N_{D}\left(\mu_{c}\right)-n^{\Gamma}\left(\mu_{c}\right)-n^{X}\left(\mu_{c}\right)\right]
$$

The chemical potential $\mu_{0}$ for a homogeneous semiconductor crystal without applied electric potential can be obtained by the charge neutrality condition $\rho\left(\mu_{0}\right)=0$. As an example the calculated charge density as a function of the position of the chemical potential, is shown in Figure A.11(a) for GaAs (n-doped 2. $10^{18} \mathrm{~cm}^{-3}$ ) and Figure A.11(b) for low doped AlAs.

For the sake of completeness, the used effective masses taken from [22] should be summarized at this point:

\begin{tabular}{l|l|l}
\hline \hline Material & GaAs & AlAs \\
\hline \hline conduction band $\Gamma$-point & $0.067 m_{0}$ & $0.15 m_{0}$ \\
\hline conduction band X-point & $0.85 m_{0}$ & $0.71 m_{0}$ \\
\hline valence band heavy hole & $0.51 m_{0}$ & $0.71 m_{0}$ \\
\hline valence band light hole & $0.082 m_{0}$ & $0.16 m_{0}$ \\
\hline \hline
\end{tabular}

Table 3: The density of states effective masses, which are used for the simulation are summarized. 
In a semiclassical approximation, the bands of the semiconductor are shifted rigidly by an energy $-\mathrm{e} V$ because of an electrostatic potential $V$. The electrochemical potential $\mu_{\mathrm{ec}}$ (in semiconductor physics also called Fermi energy $\mathrm{E}_{\mathrm{F}}$ ) is a constant in space $\mathrm{r}$ and the following relation is valid:

$$
\mu_{e c}=\mu_{c}(r)-e V(r)
$$

To obtain the charge density of the space charge region, the calculated functions for AlAs and the functions for the different doping concentrations of GaAs are introduced into the program COMSOL. Together with the geometric arrangement of the heterostructure (see Figure 4.2) the Poisson-equation is solved self consistent:

$$
\nabla^{2} V(r)=-\frac{\rho\left(\mu_{e c}+e V(r)\right)}{\varepsilon_{r} \varepsilon_{0}}
$$

For the heterostructure almost no band bending was obtained by the simulation. This result is reasonable, as the position of the chemical potential $\mu_{0}$ at $5 \mathrm{~K}$ for GaAs n-doped material of $1 \cdot 10^{16}-2 \cdot 10^{18} \mathrm{~cm}^{-3}$ are close together. The layer of intrinsic material of GaAs and AlAs is too small to screen the difference of the chemical potentials, as the number of charges for the chemical potential inside the band gap is vanishing small.

The calculated band edge profile is shown in Figure A.12. For a lateral resolved $I(V)$-spectroscopy measurement one would expect to obtain current in the colored areas. This can be compared with a differential conductivity $\mathrm{d} I / \mathrm{d} V(x, V)$-section of a large area of 200nm length shown in Figure A.13. The doping concentration in GaAs according to a topography measurement of the heterostructure is indicated at the top as the blue box and the yellow box indicates the AlAs layer. The important deviations between the measurement and the simulation are labeled and explained in the following paragraph.

For the reason of better clearness four $\mathrm{d} I / \mathrm{d} V(V)$-spectra are compared directly with the simulation in Figure A.14:

In Figure A.14(a) the tip is far away from the heterostructure, (90nm). At this distance, the measurement is equal to measurements on the free surface of Si:GaAs $\left(2 \cdot 10^{18} \mathrm{~cm}^{-3}\right)$. The main difference between the measured differential conductivity and the expected signal is the signal occurring inside the band gap (labeled (1)). This signal is well known on homogeneous GaAs; it is caused by the tip induced state, and explained in Appendix A.1.2.

In Figure A.14(b) the tip is 30nm away from the heterostructure. Approximately in this distance the low doping area of the GaAs begins. The current onset at positive voltage is shifted to higher voltage of approximately $+0.2 \mathrm{~V}$ (labeled (2)) compared to the high doped GaAs. As the effect of tip induced band bending is larger for lower doping, it seems reasonable to compare the onset with tip induced band bending simulations. Similar to the measurement at high doped GaAs one can also measure a signal inside the expected band gap, but here the measured signal just occurs at voltages lower than $-0.8 \mathrm{~V}$ (labeled (3)), between $0 \mathrm{~V}$ and $-0.8 \mathrm{~V}$ no current is measured, this shift is explained in chapter 7 . 


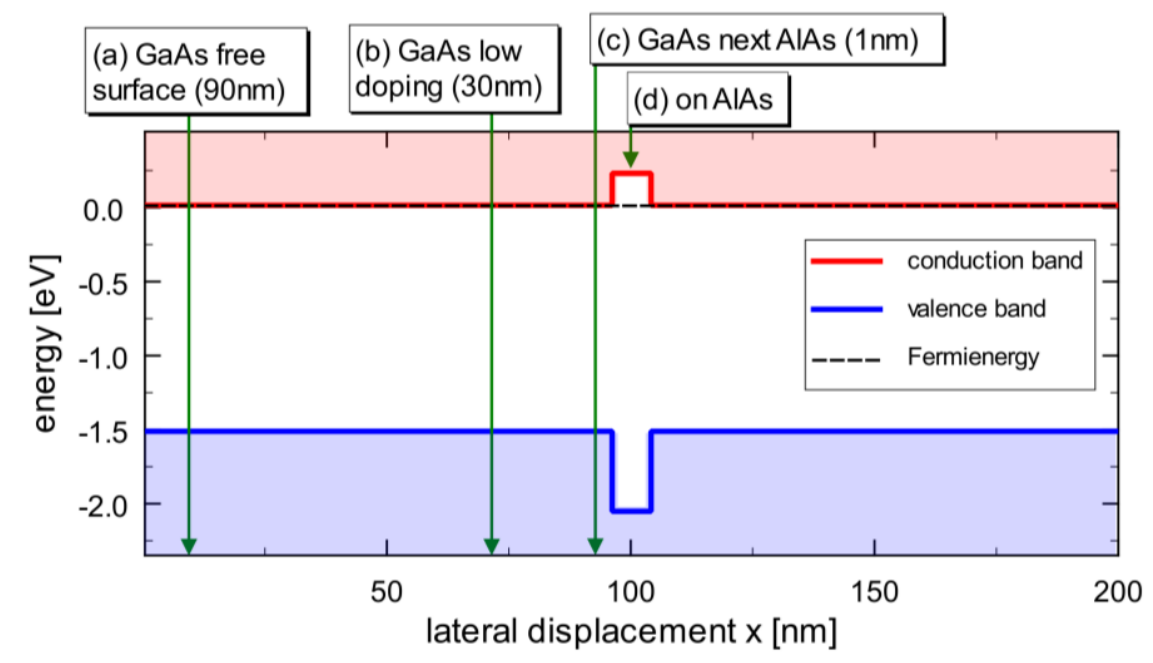

Figure A.12: Calculated band edge profile for the GaAs/AIAs/GaAs structure, including the doping gradient. The red and blue area shows where current would be expected energetically in an STM measurement. The green arrows indicate the positions where the spectroscopy is compared to the expected band structure (see Figure A.14).

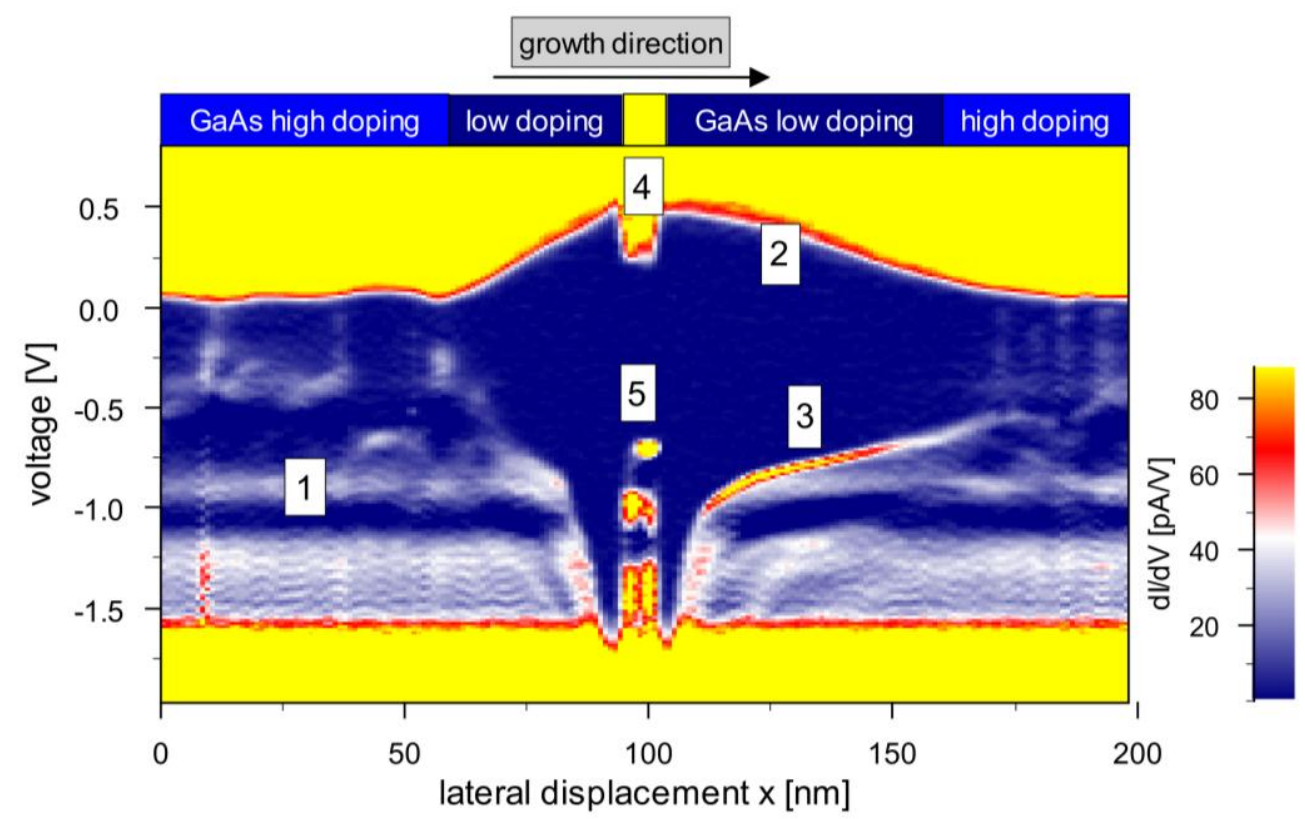

Figure A.13: A spectroscopy $d / / d V(x, V)$-section of the heterostructure is shown, the numbers are indicated in the text. In the upper part the high and low doping regime of the GaAs (blue) as well as the AlAs layer (yellow) are indicated. 

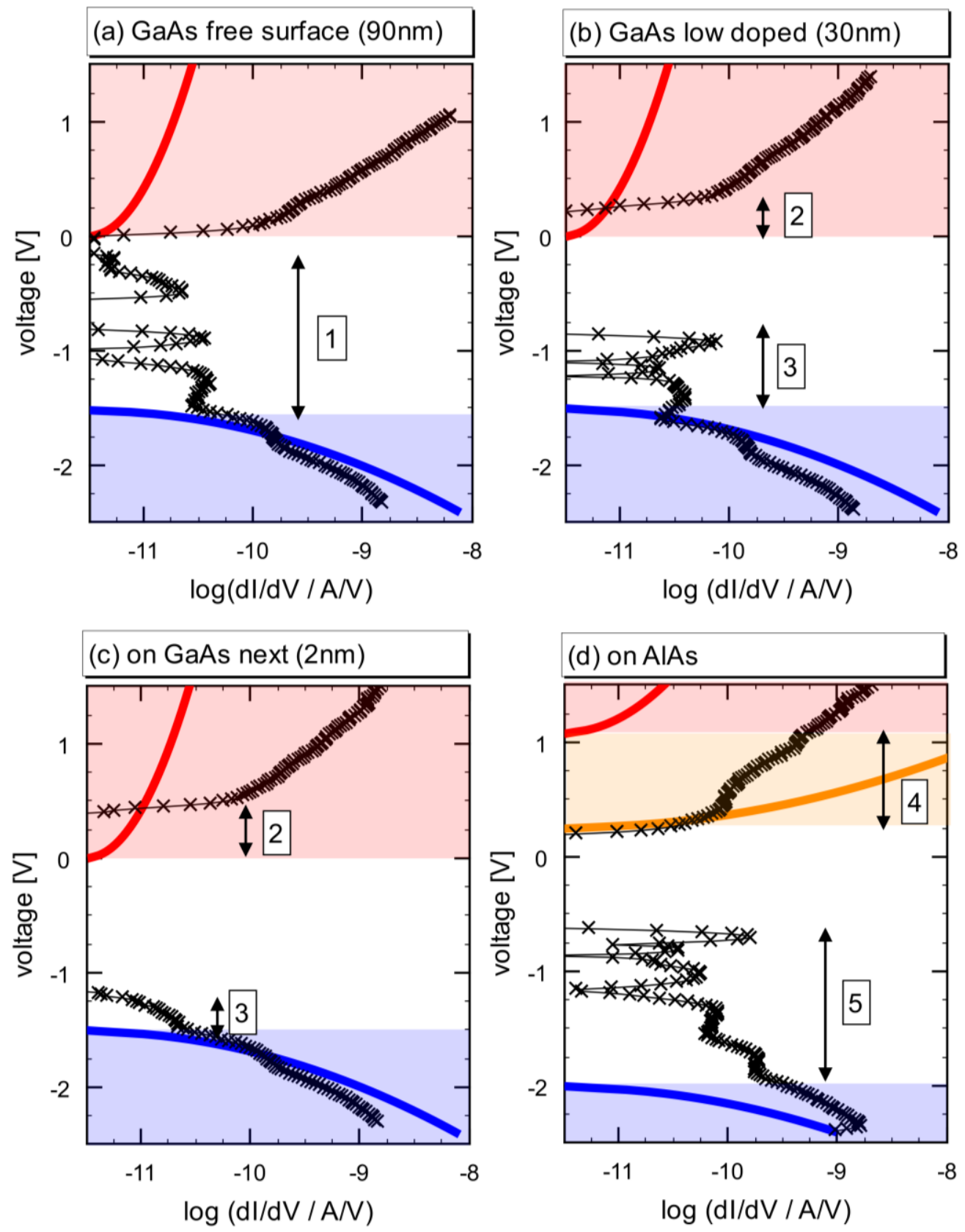

conduciton band $\Gamma$-point conduction band $\mathrm{X}$-point

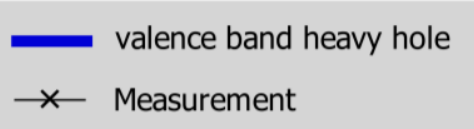

Figure A.14: Measured differential conductivity $d / / d V(V)$ (black lines) compared with the expected current. The solid lines represent the DOS of the sample in arbitrary units. The colored areas indicate the voltage range, where current can occur according to the DOS. The bands are color coded: red: conduction band ( $\Gamma$-point); orange: conduction band (X-band); blue: valence band (heavy hole). The arrows and the numbers indicate the derivation from the measurement and will be referred to in the text. 
Figure A.14(c) shows a measurement with the tip on the undoped GaAs very close to the AlAs heterostructure $(1 \mathrm{~nm})$. Here the same can be seen like in Figure A.14(b) but the effects have increased. For positive voltages the current onset is still shifted to higher voltages (labeled (2)). For negative voltages, almost the whole band gap is free of current and the peak is shifted to higher negative voltage of approximately $1.2 \mathrm{~V}$ (labeled (3)).

The $\mathrm{d} I / \mathrm{d} V(V)$-spectroscopy taken directly on AlAs is shown in Figure A.14(d). Here the largest differences from the expected signal are visible. For positive voltages the current onset starts $0.8 \mathrm{~V}$ earlier, than it would be expected for $\Gamma$-band tunneling (labeled (4)). The agreement with the X-band tunneling seems convincing, but the spectrum on AlAs cannot be seen independently from the surrounding undoped GaAs. Thus, at least a positive jump of $0.2 \mathrm{eV}$ between the onset at $1 \mathrm{~nm}$ next to the AlAs and directly on AlAs should be expected. This was discussed in section 4.2. For negative voltage in a voltage range from $-0.6 \mathrm{~V}$ to $-2 \mathrm{~V}$ current can be measured in the band gap (labeled (5)), which is discussed in section 4.3.

\section{A.2.1 The influence of the tip induced band bending}

The one dimensional simulation of the band edge profile of the heterostructure did not coincide with the measured laterally resolved differential conductivity $\mathrm{d} I / \mathrm{d} V(x, V)$ section for positive voltage. The shift of the conduction band onset to higher voltages by decreasing the distance to the heterostructure (labeled (2)) cannot be explained by the simulation. Different aspects have been neglected for that one dimensional simulation and should be discussed in this subsection.

- The surface and the influence of the tip (tip induced band bending).

The heterostructure is grown with a doping gradient. Different doping concentrations have an influence on the amount of the tip induced band bending.

- The different work functions of the two materials GaAs and AlAs.

GaAs and AlAs have a work function difference of $0.5 \mathrm{eV}$. The work function is a crucial parameter which determines the flat band condition and thus the onset voltage of the conduction band.

Both aspects are simulated including the three dimensional geometry of the tip in front of a sample, as well as the potential of the tip; they are compared in similar way to the measurements:

The expected current onset for positive voltages is estimated by the following relation. For positive TIBB a current can be expected, if the voltage is larger than the TIBB:

$$
\operatorname{TIBB}(V)<V
$$

This estimation is based on the assumption that the electrons cannot tunnel through the space charge region, which is built up by the potential of the tip. Figure A.15 should clarify the assumption. 


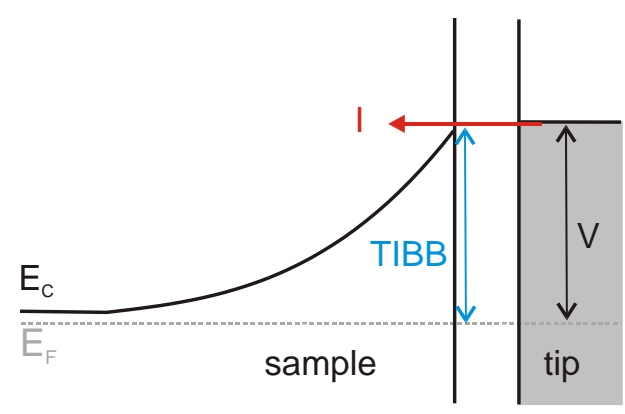

Figure A.15: A space charge layer is formed by the potential of the tip. To obtain a current the voltage $V$ has to be larger than the TIBB.
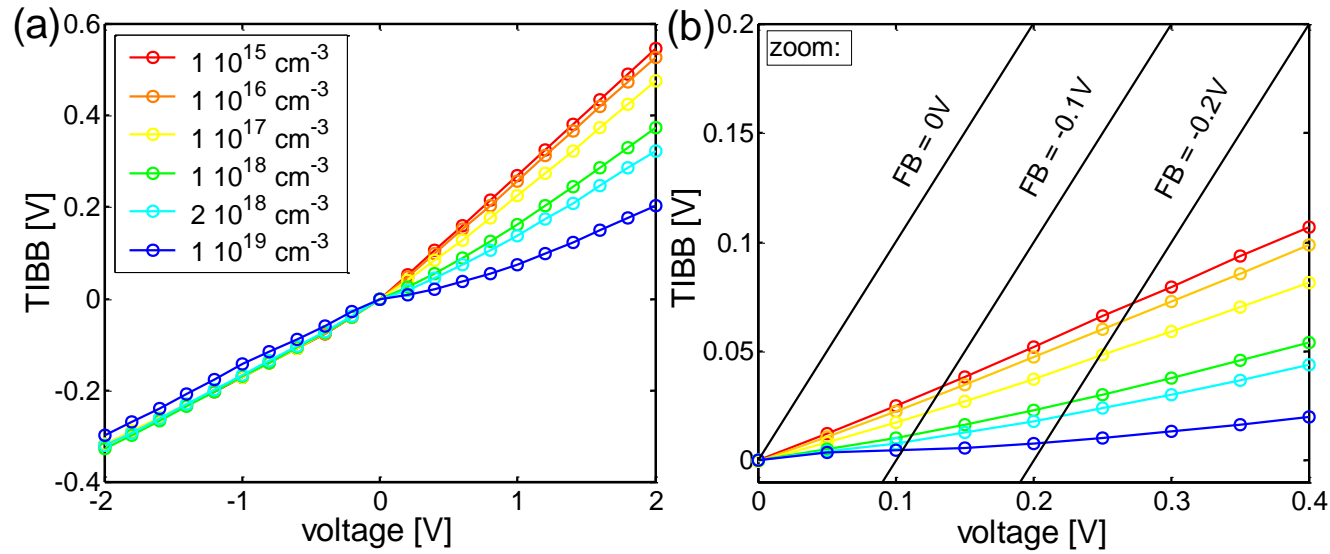

Figure A.16: Tip induced band bending for different doping concentrations. The tip radius is $5 \mathrm{~nm}$ the tip angle is $53^{\circ}$ and the tip-sample distance is $0.8 \mathrm{~nm}$. The right image is a zoom of the left image. The black line indicates the position where the voltage is equal to the TIBB. The intersection points are extracted and plotted in Figure A.17.

Starting with the aspect of different doping concentrations: The $\operatorname{TIBB}(V)$ has been simulated for a tip in front of homogeneous GaAs having different doping concentrations, the result is shown in Figure A.16(a). One important parameter of the TIBB simulation is the voltage at which the tip-sample system has flat band condition. For the simulation a shift in the flat band condition simply results in a linear shift of the tip voltage. The flat band condition can be estimated from the work function of the tip and the electron affinity of the sample. However the values cannot simply be taken, as the work function for materials are usually measured for flat surfaces, which is not valid for the curved tip. The difficulty was detailed discussed in Appendix A.1 and in [99]. They are assumed to be at $(0.0 \pm 0.2 \mathrm{~V})$.

In Figure A.16(b) a zoom of the $\operatorname{TIBB}(V)$ simulations is shown. Different flat band conditions are just represented by a shift in the voltage. The condition of equation (A.17) is visualized for different flat band conditions by the black lines. The intersection point of the black lines with the simulated TIBB is the current onset voltage. These voltages are extracted and are shown in Figure A.17 (circles); they are compared to the measurement (squares). With lower doping concentration the current 


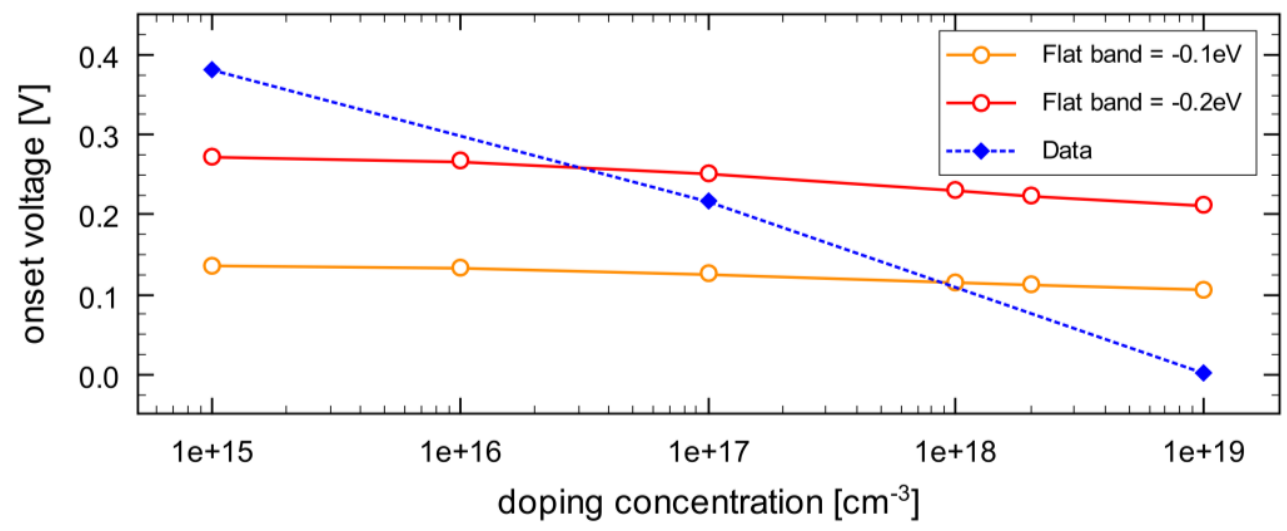

Figure A.17: Expected current onset voltage for different doping concentration extracted from the TIBB curves of Figure A.16. The current onsets for two different flat band conditions are shown. The blue squares show measured the current onset extracted from the data shown in Figure A.14.

(a) Workfunction AIAs: $3.5 \mathrm{eV}$

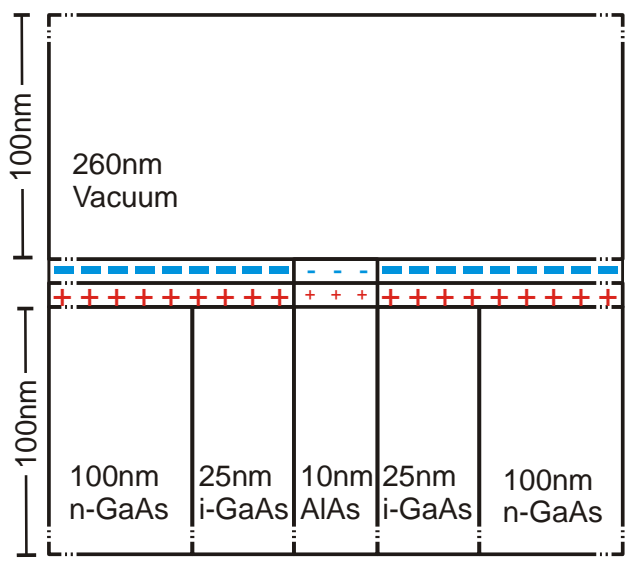

Figure A.18: (a) Geometry of the simulation for assuming a workfunction of AlAs $(3.5 \mathrm{eV})$ and $\mathrm{GaAs}(4.07 \mathrm{eV})\left(\mathrm{n}-\mathrm{GaAs}=2 \cdot 10^{18} \mathrm{~cm}^{-3}\right.$, $\mathrm{i}-\mathrm{GaAs}=$ undoped $\left.\mathrm{GaAs}\right)$. The charge amount above the AIAs layer is different to the charge amount above the GaAs layer.

onset starts at higher voltages. The trend is in accordance with the measurements, but much smaller than it would be necessary to explain the data.

The aspect of the work function difference between AlAs and GaAs is treated in the following paragraph. The geometry of the simulation is sketched in Figure A.18. The work functions at the surface are included by a dipole layer (+ positive charge, - negative charge). The amount of charge inside the dipole layer is chosen such that it will cause on a homogeneous GaAs (AlAs) sample a potential jump of $4.07 \mathrm{eV}$ $(3.5 \mathrm{eV})$. The potential landscape perpendicular to the surface is plotted in Figure A.19(a). The potential at the surface of the AlAs layer is enhanced. To clarify this observation the potential landscape at the surface ( $5 \AA$ inside the material) is plotted in x-direction in Figure A.19(b). 

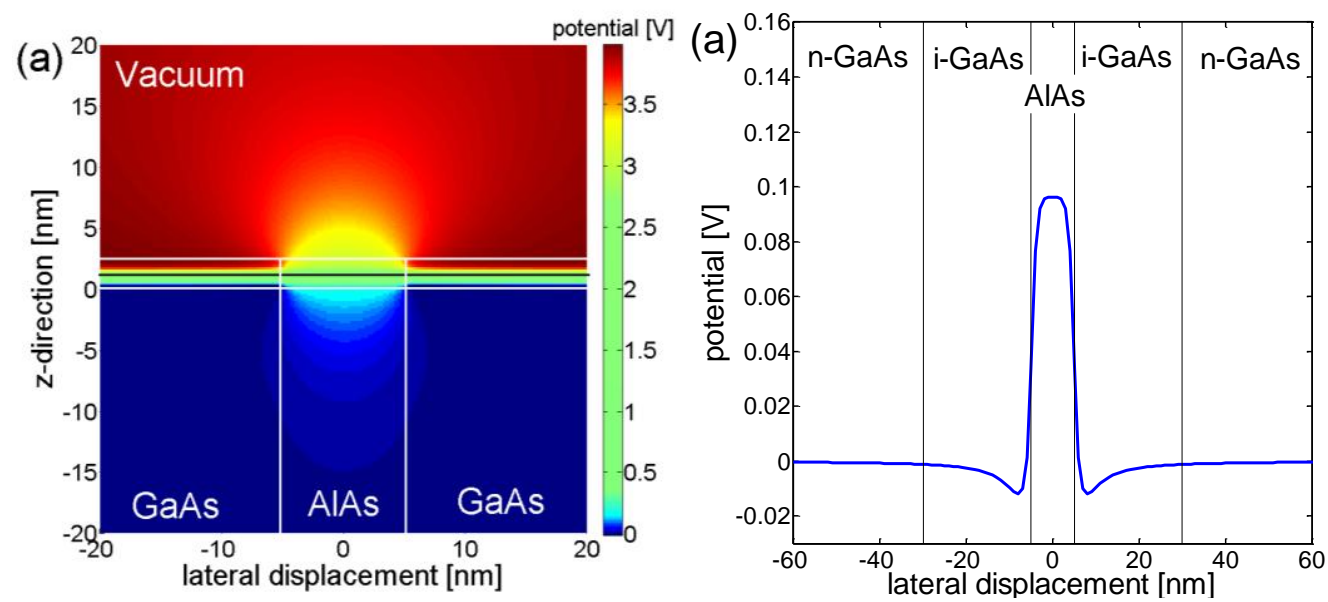

Figure A.19: (a) Potential landscape according to the simulation. The electron affinity is included by a dipole layer at the surface. The different charge amounts at the surface causes a dipole, which enhances the potential at the surface of AlAs. (b) Potential profile $0.5 \mathrm{~nm}$ inside the material without a tip.

Up to now no STM tip was included into the simulations. This was done in a next step. The geometry of the tip and the potential of the tip are included to extract $\operatorname{TIBB}(V)$ curves in different distances to the heterostructure. In Figure A.20(a) these curves are plotted. The lateral difference of the tip to the heterostructure is chosen to be $2 \mathrm{~nm}, 10 \mathrm{~nm} 20 \mathrm{~nm} 30 \mathrm{~nm}$ and $80 \mathrm{~nm}$. One curve is also shown for the tip directly in the middle of the AlAs layer. In Figure A.20 a zoom of the plot is shown, including the black line fulfilling equation (A.17). The intersection between the black line and the $\operatorname{TIBB}(V)$ curves indicate the onset value.

Figure A.21 shows the extracted intersection points (circle), which are compared to the measurements (squares). The current onset voltages are extracted from the measurement of Figure A.13. The dark blue squares are taken from the right side of the image, which is the side with the larger undoped area; the light blue squares are taken from the left side of the image, which is the side of the smaller undoped area. The different lateral extensions of the onset value are caused by the unsymmetrical doping concentration, Note that the doping concentration of the simulation was chosen to be symmetrically. The enhancement of the onset value for smaller distance to the heterostructure is visible. Even if the amount is still too less, the trend of the data is in promising agreement. 

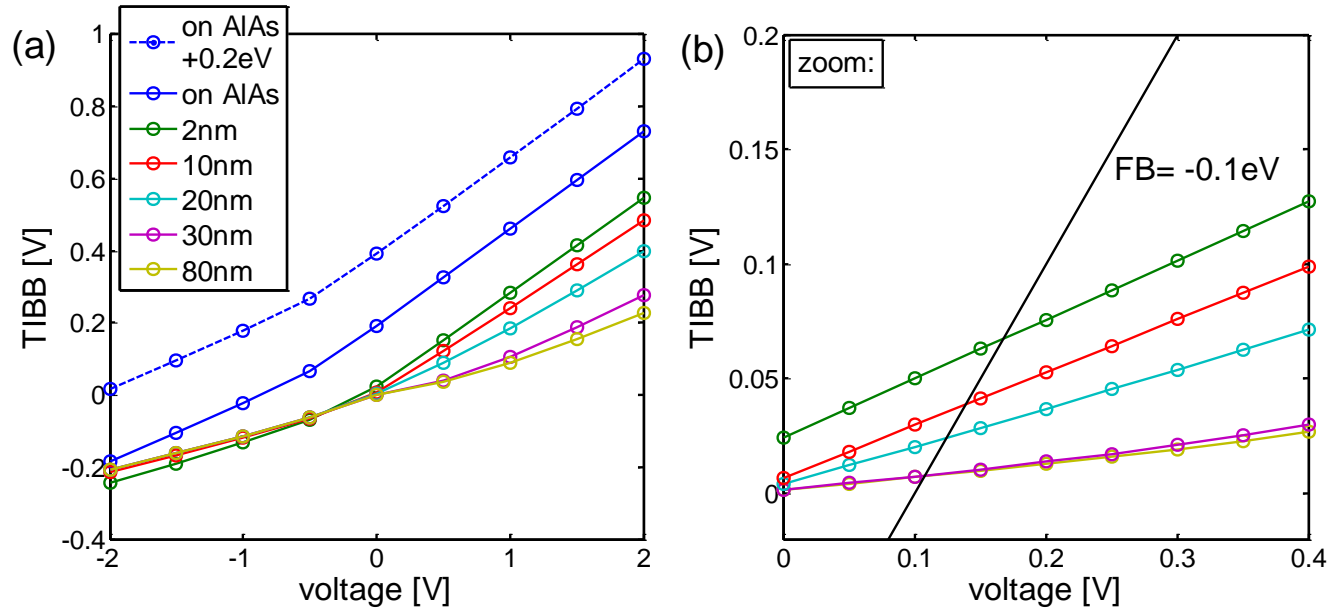

Figure A.20: TIBB as a function of voltage, for different distances from the heterostructure. The work function of AlAs is assumed to be $3.5 \mathrm{eV}$, of GaAs $4.07 \mathrm{eV}$. The dotted blue line shows a TIBB curve for AIAs shifted by the amount of $0.2 \mathrm{eV}$, due to the larger band gap of AIAs.

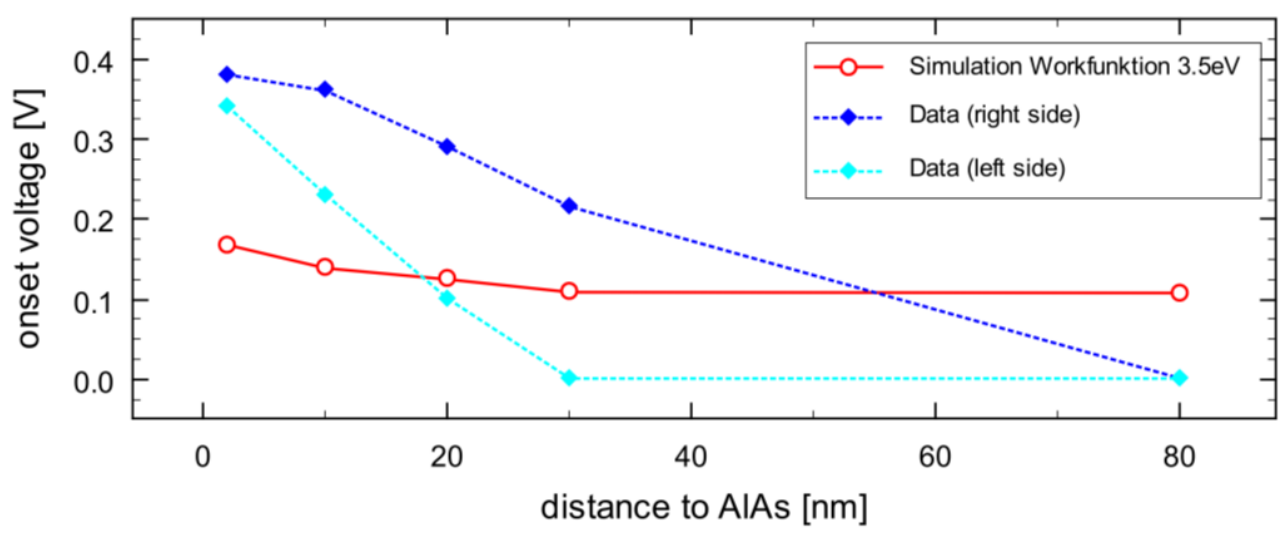

Figure A.21: Onset voltage as a function of the distance to the heterostructure for the simulation (circle) and for the measurement (squares). The data are extracted for the left and the right side of the heterostructure (in growth direction).

As a summary both aspects (different doping concentration and work function difference) explain the shift of the current onset voltage to higher voltages. The amount of the shift is too small. This could be caused by a different doping gradient, than expected from the growth conditions. It could be also possible that additionally to those two aspects an intrinsic band bending is present in the heterostructure, which lifts the bands close to the AlAs barrier. This band banding could be caused by a negative charge on or close to the heterostructure. Such a charge could for example be caused by defect states at the interface AlAs/GaAs, or it could be caused by defect states at the InAs quantum dots. As an outlook measurements on GaAs samples, which have only a doping gradient and no AlAs barrier should be done to exclude such an intrinsic band bending. 


\section{A.3 Comparison of the heterostructure samples}

Four different heterostructure samples have been investigated. Three of those samples have different extensions of the AlAs barriers including the same amount of InAs between those barriers. The fourth sample serving as a reference has no InAs quantum dots incorporated. The doping gradient is the same for all four samples. The layers of the four samples are summarized in Table 4.

\begin{tabular}{|c|c|c|c|c|}
\hline & P516 & P519 & P517 & P239 \\
\hline $\begin{array}{l}1 \mu \mathrm{m} \text { n-GaAs }\left(2 \cdot 10^{18} \mathrm{~cm}^{-3}\right) \\
10 \mathrm{~nm} \text {-GaAs }\left(1 \cdot 10^{17} \mathrm{~cm}^{-3}\right) \\
10 \mathrm{~nm} \text {-GaAs }\left(1 \cdot 10^{16} \mathrm{~cm}^{-3}\right) \\
15 \mathrm{~nm} \text { i-GaAs }\end{array}$ & & & & \\
\hline $\begin{array}{l}\text { AlAs (nm) } \\
\text { InAs (ML) }\end{array}$ & $\begin{array}{l}4 \\
1.8\end{array}$ & $\begin{array}{l}5 \\
1.8\end{array}$ & $\begin{array}{l}6 \\
1.8\end{array}$ & $\begin{array}{l}5 \\
60 \text { s growth } \\
\text { interruption }\end{array}$ \\
\hline AlAs (nm) & 4 & 5 & 6 & 5 \\
\hline $\begin{array}{l}\text { 15nm i-GaAs } \\
10 \mathrm{~nm} \mathrm{n-GaAs}\left(1 \cdot 10^{16} \mathrm{~cm}^{-3}\right) \\
10 \mathrm{~nm} \mathrm{n-GaAs}\left(1 \cdot 10^{17} \mathrm{~cm}^{-3}\right) \\
1 \mu \mathrm{m} \text { n-GaAs }\left(2 \cdot 10^{18} \mathrm{~cm}^{-3}\right) \\
\text { Substrate } \mathrm{n} \text {-GaAs }\left(2 \cdot 10^{18} \mathrm{~cm}^{-3}\right)\end{array}$ & & & & \\
\hline
\end{tabular}

Table 4: The table shows an overview of the used samples. The samples P516, P519 and P517 have InAs quantum dots between the AlAs layers. Sample P239 is a reference sample which contains no InAs.

A highly resolved constant current topography image $(30 \times 30 \mathrm{~nm} 2)$ is presented for each sample in Figure A.22; each setpoint is at negative voltage. The expected length for the AlAs layers is: Figure A.22 (a) $8 \mathrm{~nm}$ (b) $10 \mathrm{~nm}$ (c) $12 \mathrm{~nm}$ and (d) $10 \mathrm{~nm}$. The blue arrows indicate the specific length, which correspond quite well with the topographic contrast in the images. 


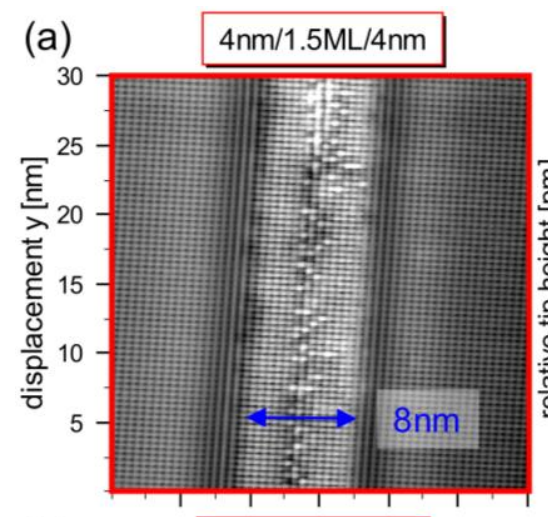

(c)

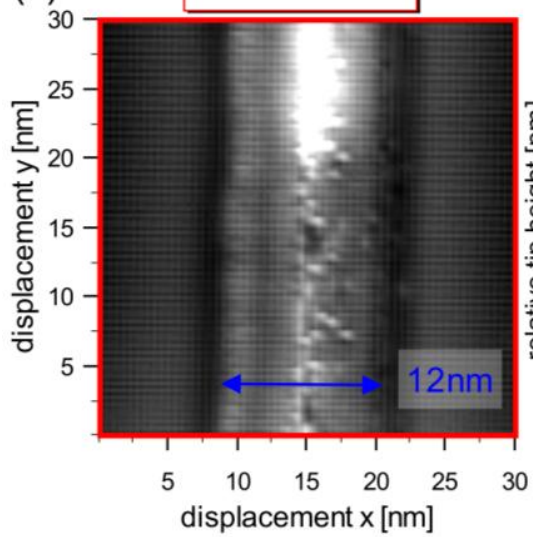

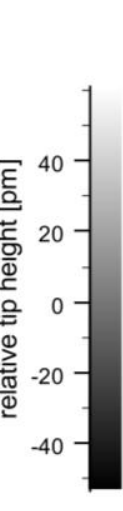

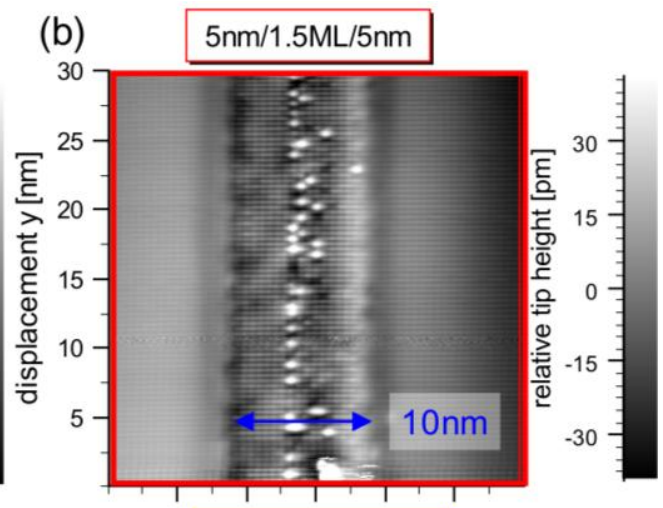

(d)

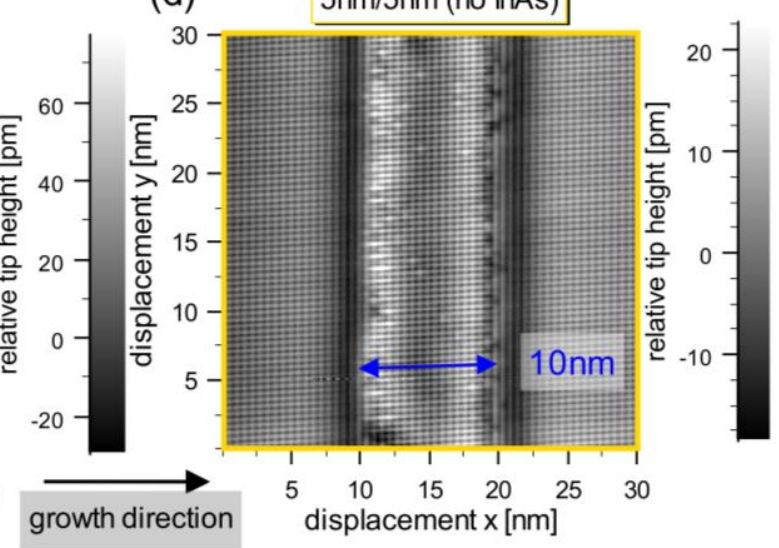

Figure A.22: Constant current topography images of the heterostructure for different samples $\left(30 \times 30 \mathrm{~nm}^{2}\right.$ ). (a) $4 \mathrm{~nm}$ AlAs, $1.8 \mathrm{ML} \operatorname{lnAs}$, 4nm AlAs, setpoint: -2V, 0.1nA (b) $5 \mathrm{~nm}$ AlAs, $1.8 \mathrm{ML}$ InAs, $5 \mathrm{~nm}$ AlAs setpoint: -2V, $0.1 \mathrm{nA}$ (c) $6 \mathrm{~nm}$ AlAs, $1.8 \mathrm{ML} \mathrm{InAs}, 6 \mathrm{~nm}$ AlAs setpoint: $-2.4 \mathrm{~V}, 0.1 \mathrm{nA}$ (d) $5 \mathrm{~nm}$ AlAs, $5 \mathrm{~nm}$ AlAs setpoint: $-2.5 \mathrm{~V}, 0.1 \mathrm{nA}$. The expected length of the AIAs layer is indicated by the blue arrow, which corresponds quite well with the topographic contrast.

The doping gradient can be resolved in a large constant current topography image across the heterostructure. Figure A.23(a) shows a $300 \times 300 \mathrm{~nm}^{2}$ constant current topography image at a voltage of $-2 \mathrm{~V}$ and a current of $0.1 \mathrm{nA}$. The AlAs layer is visible as the vertical bright line in the middle of the image. For negative voltage the donors are visible as an enhancement surrounded by a depletion (caused by Friedel oscillations) and this voltage can be used to identify the donors. In an area of $25 \times 300 \mathrm{~nm}^{2}$ the amount of visible donors is counted. The result is plotted in Figure A.23(b). The error of the donor amount is estimated to be 5 (for example adsorbates could be hiding donors or being mistakenly identified as donors). The error bar in $\mathrm{x}$ direction is meant to show the size of the counted area. The doping area on the left side of the sample is smaller $\sim 25 \mathrm{~nm}$ than on the right side of the sample $\sim 50 \mathrm{~nm}$. The donors are shifted in growth direction. The unsymmetrical doping has also been observed in transport measurements, where it has been explained by the growth process [83]. 


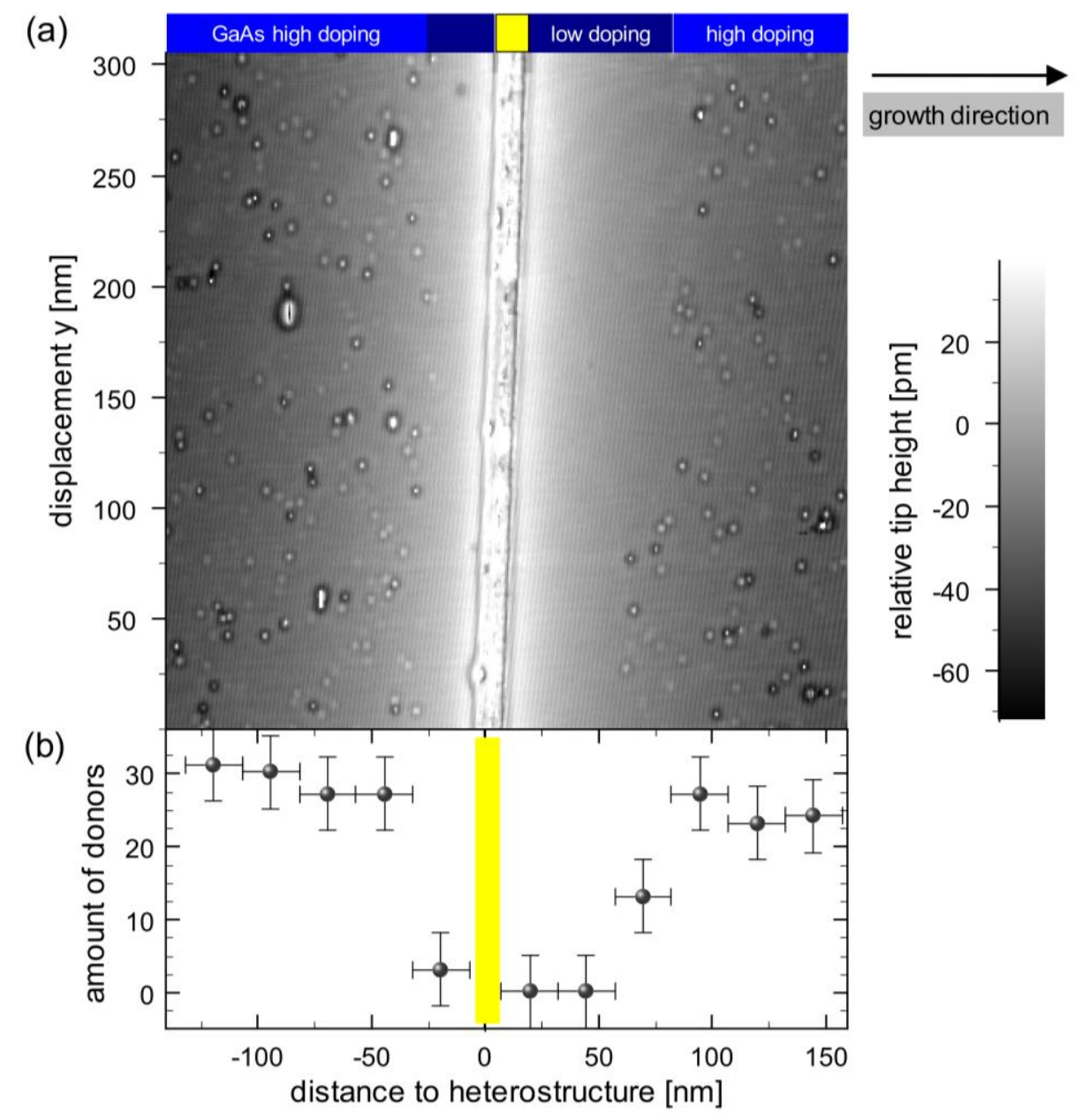

Figure A.23: (a) Constant current topography image at a setpoint of $-2 \mathrm{~V}, 0.1 \mathrm{nA}$. The heterostructure is located at $x=0 \mathrm{~nm}$. In the vicinity of the heterostructure no donors are visible. (b) In an area of $300 \times 25 \mathrm{~nm}^{2}$ the visible donors are counted and plotted as a function of distance to the heterostructure. In the region of about $30 \mathrm{~nm}$ before and about $50 \mathrm{~nm}$ after the heterostructure a low doping is measured.

The spectroscopic features of the four samples are similar. The main difference is visible for the sample without InAs. In Figure A.24 differential conductivity $\mathrm{d} I / \mathrm{d} V(x, y)$-maps $\left(30 \times 30 \mathrm{~nm}^{2}\right)$ at different voltage are shown for a sample with InAs Figure A.24(a-e) and the sample without InAs Figure A.24(f-j). The position of the AlAs/GaAs interface extracted from the corresponding topography image is marked by the green lines in each image. The voltages of the maps are chosen such that the prominent features can be compared between the two samples. For large positive voltage Figure A.24(a) and large negative voltage Figure A.24(e) enhanced differential conductivity is visible between the AlAs barriers. In the reference sample these features do not occur for large positive or negative voltages. As an example Figure A.24(f) and (j) are shown. Thus, these features are assigned to the InAs quantum dots.

The following described features are visible in both samples and are therefore not assigned to the InAs. For low positive voltage Figure A.24(b) and Figure A.24(g) enhanced differential conductivity is located on the area of the AlAs barriers, whereas 
no differential conductivity is seen on the area of the GaAs. According to the band edge this is not expected. For low negative voltage enhanced differential conductivity is located on one side of the AlAs barrier and no differential conductivity is seen on the area of the GaAs Figure A.24(c) and (h). For larger negative voltage Figure A.24(d) and (i) on both sides of the AlAs barrier differential conductivity is seen, and on the area of GaAs in a distance of about $5 \mathrm{~nm}$ next to the AlAs differential conductivity is seen as well. Also for negative voltages this sequence is not expected, as one would expect to see the differential conductivity on the area of GaAs before it appears on the area of AlAs. 


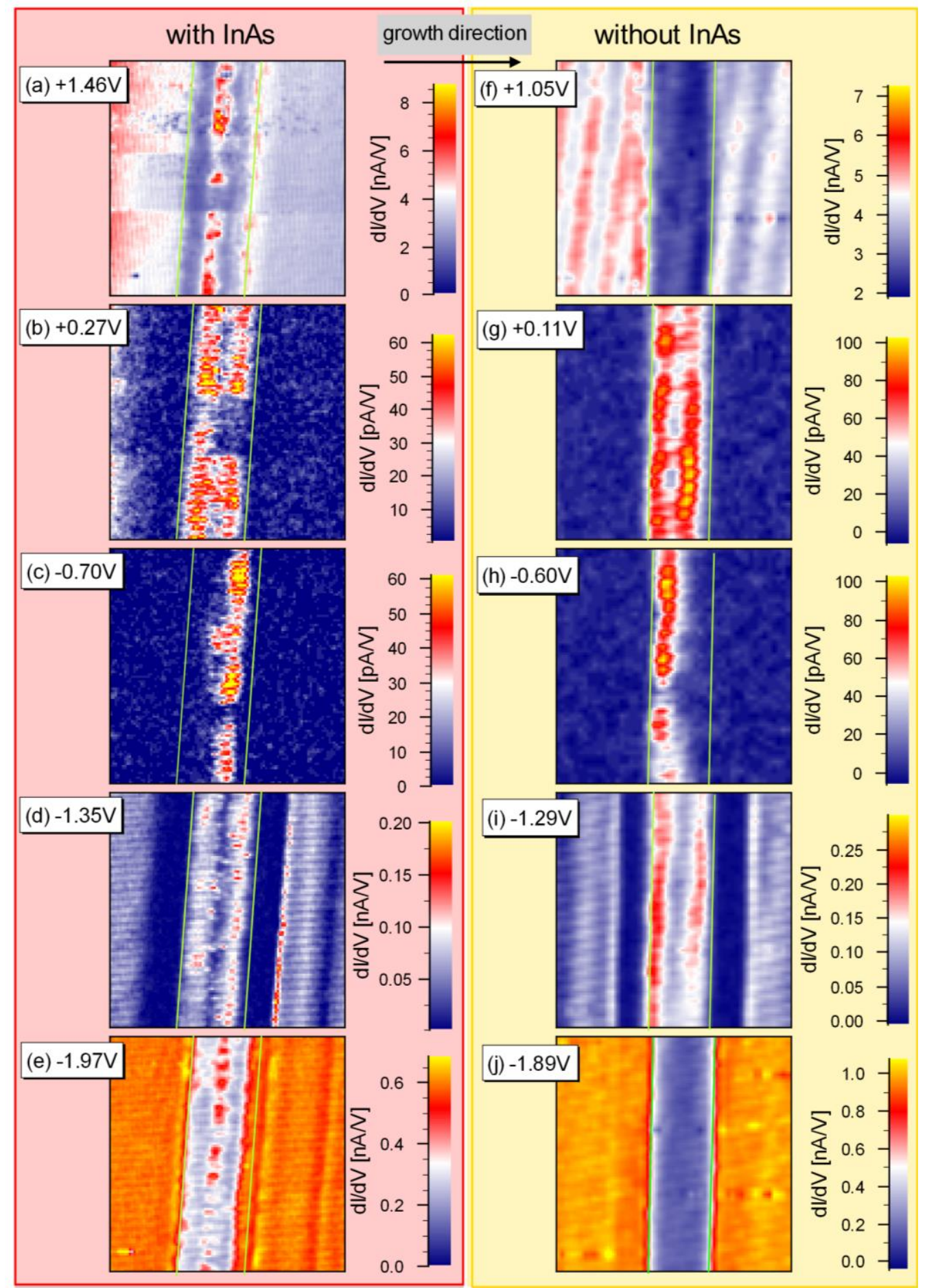

Figure A.24: differential conductivity $\mathrm{d} I / \mathrm{d} V(x, y)$-maps $\left(30 \times 30 \mathrm{~nm}^{2}\right)$ for different voltages across the heterostructure for a sample with InAs quantum dots. (a-e) and without InAs quantum dots (f-j). The voltages are chosen such that the same features are visible. The setpoint of the corresponding topography image is $-2.5 \mathrm{~V}$ and $0.5 \mathrm{nA}$ (a-e) and $-2.4 \mathrm{~V}$ and $1 \mathrm{nA}(\mathrm{f}-\mathrm{j})$. 



\section{B Bibliography and Abbreviations}

\section{B.1 Bibliography}

[1] Keyes, R. W. Appl. Phys. 8, 251 (1975).

[2] Hoeneisen, B. and Mead, C. A. Solid State Electronics 15, 819 (1972).

[3] Roy, S. and Asenov, A. Science 309, 388 (2005).

[4] Shinada, T., Okamoto, S., Kobayashi, T., and Ohdomari, I. Nature 437, 1128 (2005).

[5] Feenstra, R. M. and Stroscio, J. A. J. Vac. Sci. Technol. B 5(4), 923-929 (1987).

[6] Feenstra, R. M. J. Vac. Sci. Technol. B 21, 2080 (2003).

[7] Bardeen, J. Phys. Rev. Lett. 6(2), 57-59 Jan (1961).

[8] Binnig, G., Rohrer, H., Gerber, C., and Weibel, E. Appl. Phys. Lett. 40(2), 178180 (1982).

[9] Tersoff, J. and Hamann, D. R. Phys. Rev. B 31(2), 805-813 Jan (1985).

[10] Tersoff, J. and Hamann, D. R. Phys. Rev. Lett. 50(25), 1998-2001 Jun (1983).

[11] Hamers, J. Annu. Rev. Phys. Chem. 40, 531 (1989).

[12] Chen, C. J. Introduction to Scanning Tunneling Mecroscopy. Oxford University Press, (1993).

[13] Loth, S. Atomic scale images of acceptors in III-V semiconductors: band bending tunneling paths and wave functions. $\mathrm{PhD}$ thesis, Georg-August University of Göttingen, (2007).

[14] Lüth, H. surfaces and interfaces of solid materials. Springer-Verlag, 3rd edition, (1995).

[15] Chelikowski, J. R. and Cohen, M. L. Phys. Rev. B 20, 4150 (1979).

[16] de Raad, G. J., Bruls, D. M., Koenraad, P. M., and Wolter, J. H. Phys. Rev. B 66, 195306 (2002). 
[17] Wildöer, J. W. G., Harmans, C. J. P. M., and van Kempen, H. Phys. Rev. B 55(24), R16013-R16016 Jun (1997).

[18] Dombrowski, R., Steinebach, C., Wittneven, C., Morgenstern, M., and Wiesendanger, R. Phys. Rev. B 59(12), 8043-8048 Mar (1999).

[19] Wenderoth, M., Rosentreter, M. A., Engel, K. J., Heinrich, A. J., Schneider, M. A., and Ulbrich, R. G. Europhys. Lett. 45, 579 (1999).

[20] http://www.andrew.cmu.edu/usr/feenstra. .

[21] Chelikowsky, J. R. and Cohen, M. L. Phys. Rev. B. 14, 556 (1976).

[22] Guzzi, M. and Staehli, J. L. Solid State Phenomena 10, 25-52 (1989).

[23] Vurgaftman, I., Meyer, J. R., and Ram-Mohan, L. R. J. Appl. Phys. 89, 5815 (2001).

[24] Anderson, R. L. Solid State Electronics 5, 341 (1962).

[25] Adachi, S. GaAs and Related Materials Bulk Semiconducting and Superlattice Properties. World Scientific, Singapore, (1994).

[26] Tersoff, J. Phys. Rev. B 30, 4874 (1984).

[27] Grundmann, M. The physics of semiconductors. Springer-Verlag, (2006).

[28] Bechstedt, F. and Enderline, R. semiconductor surfaces and interfaces. Akademie-Verlag Berlin, (1988).

[29] Beres, R. P. and Allen, R. E. J. Vac. Sci. Technol. 21, 548 (1982).

[30] Beres, R. P., Allen, R. E., and D., D. J. Solid State Communications 45, 13 (1983).

[31] Vogl, P., Hjalmarson, H. P., and Dow, J. D. Journal of Physics and Chemistry of Solids 44(5), 365 - 378 (1983).

[32] Ebert, P., Engels, B., Richard, P., Schroeder, K., Blügel, S., Domke, C., Heinrich, M., and Urban, K. Phys. Rev. Lett. 77, 2997 (1996).

[33] Besocke, K. Surface Science 181(1-2), 145 - 153 (1987).

[34] Weismann, A. Scanning Tunnelling Spectroscopy of Subsurface Magnetic Atoms in Copper Electron Focusing and Kondo Effect. PhD thesis, GeorgAugust University of Göttingen, (2008).

[35] Feenstra, R. M., Stroscio, J. A., Tersoff, J., and Fein, A. P. Phys. Rev. Lett. 58(12), 1192-1195 Mar (1987).

[36] Sauthoff, K. Scanninng Tunneling Spectroscopy on Single Donors and Donor Complexes near the GaAs(110)-Surface. PhD thesis, Georg-August University of Göttingen, (2003).

[37] Schleiermacher, H. Characterization of tips for scanning tunneling microscopy. Georg-August University of Göttingen, (2006).

[38] Schubert, E. F. Doping in III-V semiconductors. Cambridge University Press, Cambridge, (1993).

[39] Hao, Y. L., Djotyan, A. P., Avetisyan, A. A., and Peeters, F. M. Phys. Rev. B 80(3), 035329 Jul (2009). 
[40] van der Wielen, M. C. M. M., van Roij, A. J. A., and van Kempen, H. Phys. Rev. Lett 76, 1075 (1996).

[41] Teichmann, K., Wenderoth, M., Loth, S., Ulbrich, R. G., Garleff, J. K., Wijnheijmer, A. P., and Koenraad, P. M. Phys. Rev. Lett. 101(7), 076103 (2008).

[42] Wildöer, J. W. G., van Roij, A. J. A., Harmans, C. J. P. M., and van Kempen, H. Phys. Rev. B 53, 10695 (1996).

[43] Repp, J., Meyer, G., Olsson, F. E., and Persson, M. Science 305, 493 (2004).

[44] Pradhan, N. A., Liu, N., Silien, C., and Ho, W. Phys. Rev. Lett. 94(7), 076801 (2005).

[45] Marczinowski, F., Wiebe, J., Meier, F., Hashimoto, K., and Wiesendanger, R. Phys. Rev. B 77(11), 115318 (2008).

[46] Brar, V. W., Decker, R., Solowan, H.-M., Wang, Y., Lorenzo, M., Chan, K. T., Lee, H., Girit, C. O., Zettl, A. Z., Louie, S. G., Cohen, M. L., and Crommie, M. F. Nature Physics 7, 43 (2011).

[47] Swart, I., Sonnleitner, T., and Repp, J. Nano Letters 11(4), 1580-1584 (2011).

[48] Besocke, K. and Wagner, H. Phys. Rev. B 8, 4597 (1973).

[49] Hahn, P., Clabes, J., and Henzler, M. J. Appl. Phys. 51(4), 2079 (1980).

[50] Schwabl, F. Quantenmechanik. Springer-Verlag, 6th edition edition, (2002).

[51] Jackson, J. D. Classical Electrodynamics. Wiley and Sons Inc. New York, (1999).

[52] Zheng, J. F., Liu, X., Newman, N., Weber, E. R., Ogletree, D. F., and Salmeron, M. Phys. Rev. Lett. 72, 1490 (1994).

[53] Depuydt, A., Haesendonck, C. V., Savinov, S., and Panov, V. Appl. Phys. A 72, 209 (2001).

[54] Wijnheijmer, A. P., Garleff, J. K., Teichmann, K., Wenderoth, M., Loth, S., Ulbrich, R. G., Maksym, P. A., Roy, M., and Koenraad, P. M. Phys. Rev. Lett. 102, 166101 (2009).

[55] Nazin, G. V., Qiu, X. H., and Ho, W. Phys. Rev. Lett. 95(16), 166103 Oct (2005).

[56] Hsu, S., Whittier, R. J., and Mead, C. A. Solid-State Electronics 13, 1055 (1970).

[57] Atkins, P. and Friedman, R. Molecular quantum mechanics. Oxford University Press, 4th edition, (2005).

[58] Tsu, R. and Esaki, L. Appl. Phys. Lett. 22, 562 (1973).

[59] Chang, L. L., Esaki, L., and Tsu, R. Appl. Phys. Lett. 24, 593 (1974).

[60] Mendez, E. E., Calleja, E., Goncaleves da Silva, C. E. T., Chang, L. L., and Wang, W. I. Phys. Rev. B 33, 7368 (1986).

[61] Bonnefoi, A. R., McGill, T. C., and Burnham, R. D. Phys. Rev. B 37, 87548762 May (1988). 
[62] Beresford, R., Luo, L. F., Wang, W. I., and Mendez, E. E. Appl. Phys. Lett. 55, 1555 (1989).

[63] Sandu, T., Chantis, A., and Iftimie, R. Phys. Rev. B 73, 075313 Feb (2006).

[64] Salemink, H. W. M., Albrektsen, O., and Koenraad, P. Phys. Rev. B 45(12), 6946-6949 Mar (1992).

[65] Gwo, S., Chao, K.-J., and Shih, C. K. Appl. Phys. Lett. 64(4), 493-495 (1994).

[66] Reusch, T. C. G., Wenderoth, M., Heinrich, A. J., Engel, K. J., Quaas, N., Sauthoff, K., Ulbrich, R. G., Weber, E. R., Uchida, K., and Wegscheider, W. Appl. Phys. Lett. 76(26), 3882-3884 (2000).

[67] Zheng, J. F., Walker, J. D., Salmeron, M. B., and Weber, E. R. Phys. Rev. Lett. 72, 2414-2417 Apr (1994).

[68] Smith, A. R., Chao, K.-J., Shih, C. K., Shih, Y. C., and Streetman, B. G. Appl. Phys. Lett. 66(4), 478-480 (1995).

[69] Wijnheijmer, A. P., Makarovsky, O., Garleff, J. K., Eaves, L., Campion, R. P., Gallagher, B. L., and Koenraad, P. M. Nano Letters 10(12), 4874-4879 (2010).

[70] Hapke-Wurst, I. Resonanter Magnetotransport durch selbstorganisierte InAs Quantenpunkte. $\mathrm{PhD}$ thesis, University of Hannover, (2002).

[71] Adachi, S. Journal of Applied Physics 66(12), 6030-6040 (1989).

[72] Loth, S., Wenderoth, M., Winking, L., Ulbrich, R. G., Malzer, S., and Döhler, G. H. Phys. Rev. Lett. 96, 066403 (2006).

[73] Bruls, D. M., Vugs, J. W. A. M., Koenraad, P. M., Salemink, H. W. M., Wolter, J. H., Hopkinson, M., Skolnick, M. S., Long, F., and Gill, S. P. A. Appl. Phys. Lett. 81(9), 1708-1710 (2002).

[74] Blokland, J. H., Bozkurt, M., Ulloa, J. M., Reuter, D., Wieck, A. D., Koenraad, P. M., Christianen, P. C. M., and Maan, J. C. Appl. Phys. Lett. 94(2), 023107 (2009).

[75] Offermans, P., Koenraad, P. M., Wolter, J. H., Pierz, K., Roy, M., and Maksym, P. A. Phys. Rev. B 72, 165332 Oct (2005).

[76] Grandidier, B., Niquet, Y. M., Legrand, B., Nys, J. P., Priester, C., Stiévenard, D., Gérard, J. M., and Thierry-Mieg, V. Phys. Rev. Lett. 85(5), 1068-1071 Jul (2000).

[77] Urbieta, A., Grandidier, B., Nys, J. P., Deresmes, D., Stiévenard, D., Lema, A., Patriarche, G., and Niquet, Y. M. Phys. Rev. B 77, 155313 Apr (2008).

[78] Girard, J., Lemaître, A., Miard, A., David, C., and Wang, Z. Z. J. Vac. Sci. Technol. B 27, 891 (2009).

[79] Gaan, S., He, G., Feenstra, R. M., Walker, J., and Towe, E. Appl. Phys. Lett. 97(12), 123110 (2010).

[80] Maltezopoulos, T., Bolz, A., Meyer, C., Heyn, C., Hansen, W., Morgenstern, M., and Wiesendanger, R. Phys. Rev. Lett. 91, 196804 Nov (2003).

[81] Nilius, N., Wallis, T. M., and Ho, W. The Journal of Physical Chemistry B 109(44), 20657-20660 (2005). 
[82] Tanaka, I., Tada, Y., Nakatani, S., Uno, K., Azuma, M., Umemura, K., Kamiya, I., and Sakaki, H. physica status solidi (c) 5(9), 2938-2940 (2008).

[83] Hapke-Wurst, I., Zeitler, U., Keyser, U. F., Haug, R. J., Pierz, K., and Ma, Z. Appl. Phys. Lett. 82(8), 1209-1211 (2003).

[84] Pierz, K., Miglo, A., Hinze, P., Ahlers, F., Ade, G., Hapke-Wurst, I., Zeitler, U., and Haug, R. physica status solidi (b) 224(1), 119-122 (2001).

[85] Kumar, A., Laux, S. E., and Stern, F. Phys. Rev. B 42, 5166-5175 Sep (1990).

[86] Muralt, P., Meier, H., Pohl, D. W., and Salemink, H. W. M. Appl. Phys. Lett. 50(19), 1352-1354 (1987).

[87] Jäger, N. D., Marso, M., Salmeron, M., Weber, E. R., Urban, K., and Ebert, P. Phys. Rev. B 67, 165307 (2003).

[88] Reusch, T. C. G., Wenderoth, M., Winking, L., Quaas, N., and Ulbrich, R. G. Phys. Rev. Lett. 93(20), 206801 Nov (2004).

[89] Reusch, T. C. G., Wenderoth, M., Winking, L., Quaas, N., and Ulbrich, R. G. Appl. Phys. Lett. 87(9), 093103 (2005).

[90] Winking, L. Untersuchung der lokalen strukturellen und elektronischen Eigenschaften von Fe-GaAs Schottky-Kontakten mit atomar aufgelöster RasterTunnel-Mikroskopie in Querschnittsgeometrie. PhD thesis, Georg-August University of Göttingen, (2009).

[91] Cobley, R. J., Teng, K. S., Brown, M. R., and Wilks, S. P. Journal of Applied Physics 102(2), 024306 (2007).

[92] Stillman, G., Wolfe, C., and Dimmock, J. Journal of Physics and Chemistry of Solids 31(6), 1199 - 1204 (1970).

[93] Aspnes, D. E. and Studna, A. A. Phys. Rev. B 27, 985-1009 Jan (1983).

[94] p’t Hooft, G. W., van der Poel, W. A. J. A., Molenkamp, L. W., and Foxon, C. T. Phys. Rev. B 35, 8281-8284 May (1987).

[95] Feenstra, R. M., Meyer, G., Moresco, F., and Rieder, K. H. Phys. Rev. B 66(16), 165204 Oct (2002).

[96] Croitoru, M., Gladilin, V., Fomin, V., Devreese, J., Kemerink, M., Koenraad, P., Sauthoff, K., and Wolter, J. Physica E: Low-dimensional Systems and Nanostructures 21(2-4), 270 - 274 (2004).

[97] Jäger, N. D., Ebert, P., Urban, K., Krause-Rehberg, R., and Weber, E. R. Phys. Rev. B 65(19), 195318 May (2002).

[98] Hopkins, B. J. and Rivière, J. C. Proceedings of the Physical Society 81(3), 590 (1963)

[99] Teichmann, K. Diplom thesis, Georg-August University of Göttingen, (2007).

[100] Bardeen, J. Phys. Rev. 49(9), 653-663 May (1936).

[101] Lang, N. D. and Kohn, W. Phys. Rev. B 3(4), 1215-1223 Feb (1971).

[102] Wijnheijmer, A. P., Garleff, J. K., Heijden, M. A. v. d., and Koenraad, P. M. J. Vac. Sci. Technol. B 28, 1086 (2010). 
[103] Teague, E. C. J. Research of the National Bureau of Standards (Reprint of a thesis at North Texas University 1978) 91, 171-23 (1986).

[104] Fließbach, T. Quantenmechanik. Spektrum Akademie-Verlag, 3rd edition, (2000).

[105] Sze, S. M. Physics of semiconductor devices. John Wiley \& Sons, Inc., (1969). 


\section{B.2 Abbreviations:}

\begin{tabular}{|c|c|}
\hline $\mathrm{d} I / \mathrm{d} V$ & differential conductivity \\
\hline $\mathrm{E}$ & energy \\
\hline $\mathrm{E}_{\mathrm{C}}$ & conduction band \\
\hline $\mathrm{E}_{\mathrm{F}}$ & Fermi energy \\
\hline $\mathrm{E}_{\mathrm{F}, \mathrm{tip}}$ & Fermi energy of the tip \\
\hline $\mathrm{E}_{\mathrm{F} \text {, sample }}$ & Fermi energy of the sample \\
\hline $\mathrm{E}_{\mathrm{F}}^{\text {left }}$ & Fermi energy of the left contact \\
\hline $\mathrm{E}_{\mathrm{F}}^{\text {right }}$ & Fermi energy of the right contact \\
\hline $\mathrm{E}_{\text {gap }}$ & Energy of the band gap \\
\hline $\mathrm{E}_{\mathrm{V}}$ & valence band \\
\hline$I$ & current \\
\hline$j$ & current density \\
\hline $\mathrm{m}^{*}$ & effective mass \\
\hline $\mathrm{m}_{0}$ & electron mass \\
\hline$V$ & voltage \\
\hline$V_{\text {Het }}$ & lateral voltage applied to the heterostructure \\
\hline$V_{\text {left }}$ & voltage applied to the left side of the sample \\
\hline$V_{\text {right }}$ & voltage applied to the right side of the sample \\
\hline$V_{\mathrm{QD}}$ & energetic position of the quantum dot state \\
\hline$V_{\text {STM }}$ & Usual tip-sample voltage \\
\hline$V_{\mathrm{I}=\mathrm{lpA}}$ & $\begin{array}{l}\text { the corresponding voltage, where the current equals } 1 \mathrm{pA} \text { (named) } \\
\text { onset voltage. }\end{array}$ \\
\hline$x, y$ & spatial coordinates parallel to the sample surface \\
\hline$z$ & spatial coordinate perpendicular to the surface \\
\hline$\phi$ & apparent barrier height \\
\hline$\mu$ & mobility \\
\hline$\mu_{\mathrm{c}}$ & chemical potential \\
\hline$\mu_{\mathrm{ec}}$ & electrochemical potential \\
\hline$\mu_{\mathrm{ec}}^{\text {left }}$ & electrochemical potential of the left contact \\
\hline$\mu_{\mathrm{ec}}^{\text {right }}$ & electrochemical potential of the right contact \\
\hline$\rho$ & charge density \\
\hline
\end{tabular}


DOS density of states

LDOS local density of states

MBE molecular beam epitaxy

STM scanning tunnelling microscopy

TIBB tip induced band bending

UHV ultra high vaccum 


\section{Danksagung}

Experimentelles wissenschaftliches Arbeiten kann man nur als Team bewältigen, daher gilt allen ein Dank, die zum Gelingen dieser Arbeit beigetragen haben:

An erster Stelle möchte ich Prof. R. G. Ulbrich danken, für die Möglichkeit dieses interessante Thema am IV. Physikalischen Institut Göttingen bearbeiten zu dürfen. Seine Herangehensweise, physikalische Probleme von grundlegender Ebene an zu verstehen, haben zum Gelingen dieser Arbeit beigetragen.

PD H. W. Schumacher von der PTB in Braunschweig möchte ich herzlich für die Übernahme des Korreferats danken.

Ein besonderer Dank für die gute Betreuung der Doktorarbeit gilt Dr. Martin Wenderoth. Die zahlreichen Diskussionen, die er immer wieder anregte waren eine enorme Bereicherung für mein physikalisches Verständnis und ohne seine fachliche sowie menschliche Kompetenz wäre diese Arbeit nie zu stande gekommen.

Bernard Spicher möchte ich für seinen Rat und Tat in allen Laborangelegenheiten danken.

Für die gute Zusammenarbeit möchte ich den Mitgliedern der Tunnelgruppe danken, Thomas Druga, Tim Iffländer, Philipp Kloth, Karolin Löser, Henning Prüser, Sergej Burbach. Sei es bei den täglichen Kaffeepausen oder auf diversen Konferenzen, ohne euch wäre die Zeit der Promotion nicht mal halb so schön gewesen. Danke Leute, wart echt ne coole Truppe.

Für die Herstellung der resonanten Tunneldioden danke ich H. W. Schumacher und K. Pierz von der PTB in Braunschweig.

Unseren Kooperationspartnern auf dem Gebiet der Si-Donatoren danke ich P. M. Koenraad, A. P. Wijnheijmer und J. Garleff von der Eindhoven University of Technology für die Diskussionen.

Außerdem möchte ich meinen Eltern Günther und Gerhild Teichmann danken, dass sie mir das Studium ermöglicht haben und sie mir somit die Möglichkeit der Promotion eröffnet haben.

Ein ganz besonderer Dank geht an Daniel Broxtermann, der mir während der ganzen Doktorarbeitszeit zur Seite gestanden hat und immer ein offenes Ohr für meine 
Probleme hatte. Ohne seine Unterstützung besonders in der letzten Phase wäre die Beendigung dieser Arbeit nicht möglich gewesen.

Meinem kleinen Sohn Arne Broxtermann möchte ich danken, weil er mich neun Monate lang täglich in die Uni begleitet hat und weil er da ist. 


\section{Lebenslauf}

\section{Karen Teichmann}

geboren am 01.10.1981 in Gießen

Staatsangehörigkeit: deutsch

vorauss. Promotion zur Erlangung des Grades eines Doktors der

Anfang 2012 Naturwissenschaften im Fach Physik

am IV. Physikalischen Institut der Univerität Göttingen

13.10.2011- Unterbrechung der Doktorarbeit wegen Mutterschutz

19.01.2012

seit 10/2007 Beginn der Doktorarbeit und wissenschaftlicher Mitarbeiter am IV. Physikalischen Institut der Univerität Göttingen

07/2007 Diplom im Fach Physik

10/2005 Beginn der Diplomarbeit

am IV. Physikalischen Institut der Univerität Göttingen

07/2003 Vordiplom im Fach Physik

10/2001 Beginn des Physikstudiums

an der Universität Göttingen

06/2001 Allgemeine Hochschulreife

an der gymnasialen Oberstufe der Liebigschule Gießen 


\section{Publikationen:}

1. Teichmann, K., Wenderoth, M., Loth, S., Garleff, J. K., Wijnheijmer, A. P., Koenraad, P. M., and Ulbrich, R. G.

Bistable Charge Configuration of Donor Systems near the GaAs(110) Surfaces

Nano Letters 11(9), 3538-3542 (2011).

2. Teichmann, K., Wenderoth, M., Loth, S., Ulbrich, R. G., Garleff, J. K., Wijnheijmer, A. P., and Koenraad, P. M.

Controlled charge switching on a single donor with a scanning tunneling microscope

Phys. Rev. Lett. 101(7), 076103 (2008).

selected as research highlight in Nature Materials 7, 764 (2008)

3. Wijnheijmer, A. P., Garleff, J. K., Teichmann, K., Wenderoth, M., Loth, S., and Koenraad, P. M.

Single Si dopants in GaAs studied by scanning tunneling microscopy and spectroscopy

Phys. Rev. B 84, 125310 Sep (2011).

4. Wijnheijmer, A. P., Garleff, J. K., Teichmann, K., Wenderoth, M., Loth, S., Ulbrich, R. G., Maksym, P. A., Roy, M., and Koenraad, P. M.

Enhanced Donor Binding Energy Close to a Semiconductor Surface

Phys. Rev. Lett. 102, 166101 (2009).

5. Loth, S., Wenderoth, M., Teichmann, K., and Ulbrich, R. G.

Band structure related wave-function symmetry of amphoteric Si dopants in GaAs

Solid State Communications 145, 551 (2008).

\section{Präsentationen:}

6. K. Teichmann, M. Wenderoth, R. G. Ulbrich, K. Pierz, and H. W. Schumacher Investigation of an operating resonant tunneling device by scanning tunneling spectroscopy

Frühjahrstagung der DPG 2011 in Dresden

7. K. Teichmann, M. Wenderoth, S. Loth, R. G. Ulbrich, J. K. Garleff, A. P. Wijnheijmer, P. M. Koenraad

Charge switching of donor systems near the $\operatorname{GaAs}(110)$ surface induced by the tip of an STM

International Conference on Nanoscience and Technology (ICN+T 2010) in Beijing 
8. K. Teichmann, M. Wenderoth, S. Burbach, R. G. Ulbrich, K Pierz, and H. W. Schumacher

Cross-Section Scanning Tunneling Spectroscopy on a resonant-tunneling diode structure

Frühjahrstagung der DPG 2010 in Regensburg

9. K. Teichmann, M. Wenderoth, S. Loth, R. G. Ulbrich, J. K. Garleff, A. P. Wijnheijmer and P. M. Koenraad

Charge Switching of Donor Ensembles in a Semiconductor

Frühjahrstagung der DPG 2010 in Regensburg

10. K. Teichmann, M. Wenderoth, S. Loth and R. G. Ulbrich

Wave function imaging and resonant tunneling for $\mathrm{C}$ acceptors in GaAs

5th International Symposium on Scanning Probe Spectroscopy and Related Methods in Wasowo Palace (2009)

11. K. Teichmann, M. Wenderoth, S. Loth, R. G. Ulbrich, J. K. Garlef, A. P. Wijnheijmer and P. M. Koenraad

Control of Donor Charge States with the Tip of a Scanning Tunnelling Microscope

Frühjahrstagung der DPG 2009 in Dresden

12. K. Teichmann, M. Wenderoth, S. Loth, R.G. Ulbrich, J.K. Garleff, A.P. Wijnheijmer, Paul Koenraad

Tip-Induced Charge Switching and Mapping of Electrostatic Potentials in Donor Ensembles

International Conference on Nanoscience + Technology (ICN+T 2008) in Keystone.

13. K. Teichmann, M. Wenderoth, S. Loth, R. G. Ulbrich, J. Garlef, A. P. Wijnheijmer, and P. M. Koenraad

Manipulation of charge on a single donor atom by scanning tunneling microscopy

Frühjahrstagung der DPG 2008 in Berlin

14. K. Teichmann, M. Wenderoth, S. Loth, R. G. Ulbrich

Build up of a 7T-STM for investigation of spin filter effects using evanescence states

Sommerschule "Semiconductor Spinelectronics" des Schwerpunktprogramm SPP 1285 in Goslar (2008)

15. K. Teichmann, M. Wenderoth, S. Loth, and R. G. Ulbrich

Scanning Tunneling Spectroscopy of Si donors in GaAs $\{110\}$

Frühjahrstagung der DPG 2007 in Regensburg

16. S. Loth, M. Wenderoth, K. Teichmann, R. G. Ulbrich, and U. Kretzer

Spatial fluctuations of the local potential in Silicon doped GaAs

Frühjahrstagung der DPG 2006 in Dresden 
